\title{
WestVirginiaUniversity
}

THE RESEARCH REPOSITORY @ WVU

Graduate Theses, Dissertations, and Problem Reports

2015

\section{Erbium alloyed AIN thin films: Structural, piezoelectric and magnetic properties}

Vishal Narang

Follow this and additional works at: https://researchrepository.wvu.edu/etd

\section{Recommended Citation}

Narang, Vishal, "Erbium alloyed AIN thin films: Structural, piezoelectric and magnetic properties" (2015). Graduate Theses, Dissertations, and Problem Reports. 6293.

https://researchrepository.wvu.edu/etd/6293

This Dissertation is protected by copyright and/or related rights. It has been brought to you by the The Research Repository @ WVU with permission from the rights-holder(s). You are free to use this Dissertation in any way that is permitted by the copyright and related rights legislation that applies to your use. For other uses you must obtain permission from the rights-holder(s) directly, unless additional rights are indicated by a Creative Commons license in the record and/ or on the work itself. This Dissertation has been accepted for inclusion in WVU Graduate Theses, Dissertations, and Problem Reports collection by an authorized administrator of The Research Repository @ WVU.

For more information, please contact researchrepository@mail.wvu.edu. 


\title{
Deep Features based Hierarchical Classification Scheme for Face Recognition in Heterogeneous Environments
}

\author{
Neeru Narang \\ Dissertation submitted to the \\ Benjamin M. Statler College of Engineering and Mineral Resources \\ at West Virginia University \\ in partial fulfillment of the requirements \\ for the degree of
}

Doctor of Philosophy

in

Computer Engineering

Thirimachos Bourlai, Ph.D., Chair

Katerina Goseva-Popstojanova, Ph.D.

Yuxin Liu, Ph.D.

Jeremy Dawson, Ph.D.

Jacqueline A. Speir, Ph.D.

Lane Department of Computer Science and Electrical Engineering

Morgantown, West Virginia

2017

Keywords: Heterogeneous Face Recognition, Deep Learning, Convolution Neural Network, Image Restoration, Score Level Fusion Scheme, Statistical Analysis Test 


\section{Abstract \\ Deep Features based Hierarchical Classification Scheme for Face Recognition in Heterogeneous Environments}

Neeru Narang

This dissertation investigates the advantages and limitations of the heterogeneous problem of matching infrared long-range, night time face images against their visible counterparts. The contributions of the thesis are three-fold. First, a multi-feature scenario dependent fusion scheme is developed, where Gabor Wavelets, Histogram of gradients (HOG) and Local binary patterns (LBP) feature descriptors are empirically selected. Next, a set of fusion score level schemes are developed and applied before face matching. The developed fusion scheme results in 54 percent point (pp) higher recognition performance than the baseline established using a commercial and a set of academic face matchers. Second, to further improve the performance of baseline face recognition (FR) systems, a scenario dependent and sensor adaptable convolutional neural network $(\mathrm{CNN})$ is developed that groups the data in terms of demographic information, including scenarios (situations) such as indoors or outdoors data, as well as distance and sensor based data. The automated grouping scheme developed is applied before the FR algorithms are used, improving baseline performance from $48 \%$ (all vs. all) to $70 \%$ (with data grouping). Third, an image quality restoration scheme is designed and developed. This scheme is beneficial to FR systems because the quality of face data captured under challenging conditions is affected by a variety of noise factors (including low illumination conditions, variable standoff distances, and uncooperative subjects). Thus, image quality is responsible for the performance degradation of conventional FR matchers. The developed scheme improves, first, the quality of distorted face images and, then, FR performance in terms of the rank- 1 identification rate. Based on the experimental results the major conclusion from this research is that the aforementioned schemes discussed in this dissertation significantly improve cross-spectral face matching performance on diverse scenarios, when used either independently or in combination. The experimental results are further supported by statistical analysis tests, conducted to find the statistical significance of incorporating the developed score level fusion schemes, as well as the demographic filtering to FR systems. 
I dedicate my thesis to my family 


\section{Acknowledgments}

I would like to use this opportunity to thank people who helped me in completing the research presented in this dissertation.

First, and foremost of all, I would like to express my deepest appreciation and gratitude to my advisor and committee chair, Dr. Thirimachos Bourlai for giving me this opportunity. This dissertation could not have been completed without his constant guidance, endless encouragement and continuous support. His knowledge, dedication and hardworking nature has inspired me in many ways. Working with him gave me the opportunity to learn and to achieve my goals. I consider it as a privilege to have him as my advisor for this work.

I also wish to express my sincere thanks to the members of my committee, Dr. Katerina Goseva-Popstojanova, Dr. Yuxin Liu, Dr. Jeremy Dawson and Dr. Jacqueline A. Speir for serving as my committee members. The valuable guidance and suggestions from all of them helped me to improve the quality of my dissertation.

I am deeply thankful to my parents for their support, love and for all the sacrifices they made for me. A very special thanks to my husband Vishal Narang, for his unconditional love, constant support and motivation during my $\mathrm{PhD}$. I would not have made it this far without his encouragement, understanding and patience. Thank you for always being with me in my all successes and failures. I am thankful to my sister who has always been a source of inspiration for me.

I would like to extend my thanks to all my friends and colleagues: Dr. Cameron Whitelam, Nnamdi Osia, Michael Martin, Xuan Xu, Yixin Du, Erin Moore, Rohitha Reddy Matta and Suha Reddy Mokalla for their co-operation and support. 


\section{Contents}

$\begin{array}{lll}1 \text { Introduction } & 1\end{array}$

1.1 Face Recognition Systems $\ldots \ldots \ldots \ldots$. . . . . . . . . . . 1

1.2 Challenges for Face Recognition $\ldots \ldots \ldots \ldots \ldots$

1.2.1 General Research Challenges . . . . . . . . . . . . . . . 4

1.2.2 Focused Research Challenges . . . . . . . . . . . . . . . . 4

1.3 Research Objective $\ldots \ldots \ldots \ldots \ldots \ldots$

1.3 .1 Contributions . . . . . . . . . . . . . 5

1.4 Thesis Organization $\ldots \ldots \ldots \ldots \ldots \ldots \ldots$

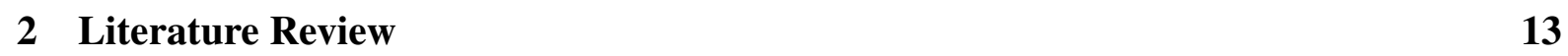

2.1 Heterogeneous Face Matching . . . . . . . . . . . . . . 13

2.1 .1 Reported Work . . . . . . . . . . . . . . . . . . . . 13

2.1 .2 Developed Solution for Heterogeneous Face Matching System . . . . . 15

2.2 Demographic Filtering $\ldots \ldots \ldots \ldots \ldots$

2.2 .1 Reported Work $\ldots \ldots \ldots \ldots$

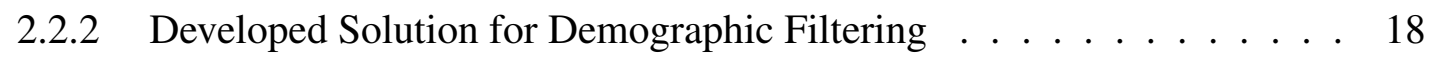

2.3 Image Filtering . . . . . . . . . . . . . . . . . . . . 19

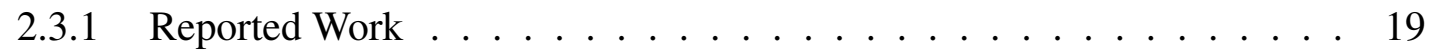

2.3 .2 Developed Solution for Image Filtering . . . . . . . . . . . . 20

2.4 Statistical Hypothesis Testing for Face Matching $\ldots \ldots \ldots \ldots$

2.4 .1 Reported Work . . . . . . . . . . . . . . . . . 21

2.4 .2 Developed Solution . . . . . . . . . . . . . . . . . 22 
3 West Virginia University Heterogeneous Database 23

$3.1 \quad$ Night Time Imagery Long Distance NIR Database . . . . . . . . . . . . . . . . 23

3.2 Multi-Scenario SWIR Database $\ldots \ldots \ldots \ldots \ldots$

$3.3 \quad$ Single Sensor Multi-Wavelength SWIR Database $\ldots \ldots \ldots$. . . . . . . . . . . 27

3.4 Multi-Sensor VIS Database $\ldots \ldots \ldots \ldots$

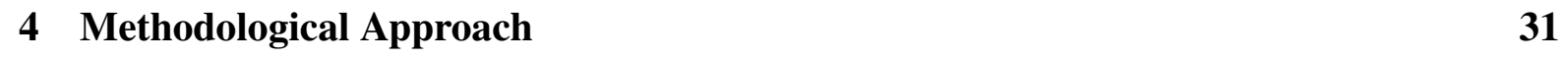

$4.1 \quad$ Study1: Developed Multi-Feature Scenario Dependent Fusion Method . . . . . 31

$4.1 .1 \quad$ Pre-processing . . . . . . . . . . . . . . . 31

4.1 .2 Feature Extraction $\ldots \ldots \ldots \ldots \ldots$

4.1 .3 Feature Subspace . . . . . . . . . . . . . . . . 34

4.1 .4 Fusion of scores $\ldots \ldots \ldots \ldots$

4.2 Study 2: Developed CNN Network for Automatic Prediction of Demographic Information . . . . . . . . . . . . . . . . 37

$4.2 .1 \quad$ Network Architecture . . . . . . . . . . . . . . . . . . . . 38

4.3 Study 3: Developed Image Restoration Scheme . . . . . . . . . . . . . . . . . 39

5 Multi-Feature Scenario Dependent Fusion Scheme for Night Time Long Range

$\begin{array}{ll}\text { Imagery System in NIR Band } & 44\end{array}$

$5.1 \quad$ Baseline Face Recognition Tools . . . . . . . . . . . . . . . . . . . . 44

5.2 Face Recognition Studies $\ldots \ldots \ldots$. . . . . . . . . . . . . 45

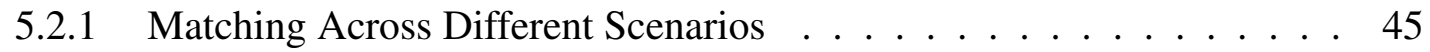

5.2.2 Baseline and Developed System Matching Results Before Demographic Filtering . . . . . . . . . . . . . . . . . 47

5.3 Conclusion from Study 1 for NIR Database $\ldots \ldots \ldots$. . . . . . . . 55

5.4 Threats to Validity $\ldots \ldots \ldots \ldots \ldots \ldots \ldots$

5.4 .1 Construct Validity $\ldots \ldots \ldots \ldots \ldots$

5.4 .2 Internal Validity $\ldots \ldots \ldots \ldots \ldots$

5.4 .3 Conclusion Validity $\ldots \ldots \ldots \ldots \ldots$. . . . . . . . . . 57

$5.4 .4 \quad$ External Validity $\ldots \ldots \ldots \ldots$ 
6 CNN based Demographic Filtering for Night Time Long Range Imagery System

in NIR Band

6.1 Demographic Filtering from developed CNN Architecture $\ldots \ldots . . . . .58$

6.1 .1 Training and Testing $\ldots \ldots \ldots \ldots \ldots$

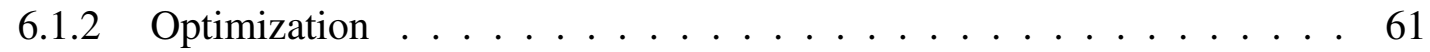

6.1 .3 Demographic Filtering Results for Ethnicity Class . . . . . . . . . . 63

6.1 .4 Demographic Filtering Results for Gender Class ～. . . . . . . . . . . 65

6.1.5 Demographic Filtering Results for Male Class into With or Without Beard 66

6.1 .6 Demographic Filtering Results for Female Class into Asian or Caucasian 68

6.2 Face Matching based on Demographic Information $\ldots \ldots \ldots$

6.2 .1 Demographic-based Cross-Distance Matching. . . . . . . . . . . . . . 69

6.2 .2 Demographic-based Cross-Spectral Matching . . . . . . . . . . . 72

$6.2 .3 \quad$ Face Matching With Extended Gallery Data . . . . . . . . . . . . . 76

6.3 Conclusion From Study 3 . . . . . . . . . . . . . . . . . . . 80

6.3.1 Intra-spectral and Cross-Distance using Developed Approach. Normalized images are used to perform the experiments. . . . . . . . . . 80

6.3.2 Cross-spectral and Cross-Distance: Using Developed Approach . . . . 80

6.3.3 Cross-spectral and Cross-Distance for Male Class: COTS vs. Academic vs. Developed Approach f . . . . . . . . . . . . . . . . . 81

6.4 Threats to Validity $\ldots \ldots \ldots \ldots \ldots \ldots$. . . . . . . . . . . . . 81

$6.4 .1 \quad$ Construct Validity $\ldots \ldots \ldots \ldots \ldots$. . . . . . . . . . . . 81

6.4 .2 Internal Validity $\ldots \ldots \ldots \ldots \ldots$. . . . . . . . . . 82

6.4 .3 Conclusion Validity $\ldots \ldots \ldots \ldots$. . . . . . . . . . 83

$6.4 .4 \quad$ External Validity $\ldots \ldots \ldots \ldots$. . . . . . . . . . . . . 83

7 Developed Image Restoration Scheme for Night Time Long Range Imagery Sys$\begin{array}{ll}\text { tem in NIR Band } & 85\end{array}$

$7.1 \quad$ Baseline Face Matching Results using developed Image Restoration . . . . . . 85

7.2 Matching using developed Image Restoration and Fusion Scheme . . . . . . . 86 
7.3 Matching using developed Image Restoration, Fusion Scheme and Demographic

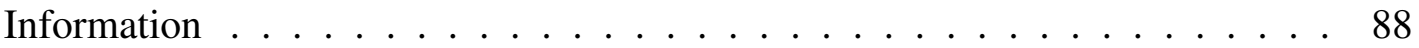

7.4 Conclusion from Study $3 \ldots \ldots \ldots$. . . . . . . . . . . . . 93

7.4.1 Comparison between Developed Study 1 vs. Academic and Commercial Matcher . . . . . . . . . . . . . . . . 93

7.4.2 Comparison between Developed Study 1 vs. Study 1 (Fusion Scheme)

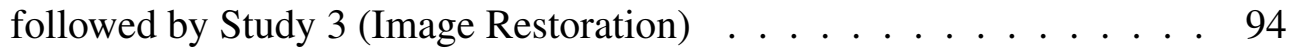

7.5 Threats to Validity $\ldots \ldots \ldots \ldots \ldots \ldots$. . . . . . . . . . . . . 95

7.5 .1 Construct Validity . . . . . . . . . . . . . . 95

7.5 .2 Internal Validity $\ldots \ldots \ldots \ldots$. . . . . . . . . . . 96

7.5 .3 Conclusion Validity $\ldots \ldots \ldots \ldots$. . . . . . . . . . . 96

7.5 .4 External Validity $\ldots \ldots \ldots$. . . . . . . . . . . . . . 96

8 Study 4: Statistical Analysis $\quad 98$

$8.1 \quad$ Statistical Hypothesis Testing $\ldots \ldots \ldots \ldots$. . . . . . . . . . 98

8.2 Selection of Statistical Test $\ldots \ldots$. . . . . . . . . . . . . 100

$8.2 .1 \quad$ Independence/Dependence of Samples . . . . . . . . . . . . . . 101

$8.2 .2 \quad$ Statistical Test to find Distribution . . . . . . . . . . . . . . . . . 101

8.2.3 Statistical Tests to check Homogeneity of Variance . . . . . . . . . . . 102

8.3 Statistical Hypothesis Test $1 \ldots$. . . . . . . . . . . . . . 103

8.4 Statistical Hypothesis Testing 2 $\ldots \ldots$. . . . . . . . . . . . . . 108

8.5 Statistical Hypothesis Test 3 . . . . . . . . . . . . . . . . . . . . 109

8.6 Conclusion from Study $4 \ldots \ldots \ldots \ldots \ldots$. . . . . . . . . . . . . . . .

9 Multi-Scenario and Multi-Wavelength SWIR based Facial Recognition System 112

9.1 Classification and Face Matching for Multi Scenario Database . . . . . . . 113

$9.1 .1 \quad$ Classification from Developed CNN Architecture . . . . . . . . . . . . 113

9.1 .2 Cross-scenario Face Matching Results . . . . . . . . . . . . . . . . 120

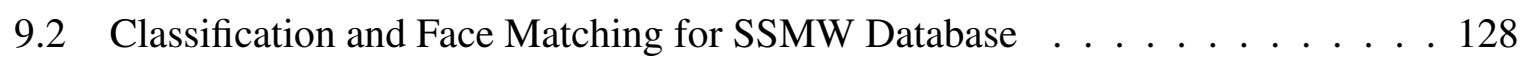

$9.2 .1 \quad$ Classification using Developed CNN Architecture $\ldots . . . . . .130$ 
9.2 .2 Face Matching for SSMW Database . . . . . . . . . . . . . . . 134

9.2 .3 Frontal and Non-Frontal View Face Classification . . . . . . . . . . . . 134

9.2 .4 Normalization of Data $\ldots \ldots \ldots$. . . . . . . . . . . . . 134

9.2.5 Cross-Spectral Face Matching based on developed MFSDF Scheme . . 135

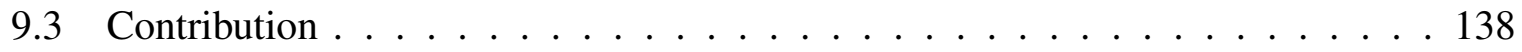

$9.3 .1 \quad$ Evaluation for Multi-Scenario Database . . . . . . . . . . . . . . . . 138

9.3.2 Evaluation for Single Sensor Multi-Wavelength Database . . . . . . . . 140

10 Multi-Sensor based Facial Recognition System 142

10.1 Classification from Developed CNN Architecture . . . . . . . . . . . . . . . . 143

10.1 .1 Training and Testing . . . . . . . . . . . . . . . . 145

10.1.2 Level 1: CNN based Phone Type Classification . . . . . . . . . . . . . 145

10.1.3 Level 2: CNN based Conditional Classification . . . . . . . . . . . . . 147

10.1.4 Level 3: CNN based Standoff Distance Classification . . . . . . . . . . 148

10.2 Face Matching Results: With and Without Grouping . . . . . . . . . . . 150

10.2.1 Classification of Frontal vs. Non-Frontal . . . . . . . . . . . . . 150

10.2 .2 Face Matching . . . . . . . . . . . . . . . . 153

10.2.3 Face Matching Results: With and Without Grouping . . . . . . . . 153

10.2.4 Classification and Matching: With Extended Database . . . . . . . . 155

10.3 Conclusion . . . . . . . . . . . . . . . . . . . 155

\begin{tabular}{ll}
\hline 1 Dissertation Conclusions & 158
\end{tabular}

11.1 Conclusions $\ldots \ldots \ldots \ldots \ldots \ldots \ldots$

11.2 Future Work . . . . . . . . . . . . . . . . . . . . . . . . 162 


\section{List of Figures}

$1.1 \quad$ Block diagram representation of a typical face recognition system. . . . . . . . . 2

1.2 Mixed face recognition scenarios involve matching probe images captured by any camera sensor, standoff distance and illumination conditions. . . . . . . . . . . . . 3

$1.3 \quad$ Face images collected at standoff distance of $5 \mathrm{~m}$ and $10 \mathrm{~m} . \ldots \ldots \ldots$

1.4 Data collection scenarios of both visible and infrared face data, under controlled (top image) or uncontrolled (bottom image) conditions [7]. . . . . . . . . . . . . 6

1.5 Probe face images are captured in the SWIR band through tinted glass. . . . . . . . 6

1.6 Overview of conventional face recognition system using developed pipeline (top box) and incorporation of statistical tests for the verification purpose. $\ldots \ldots$. . . . . 8

3.1 Overview of the NIR camera system with the capability to acquire face images day and night at mid-ranges, i.e., from about 20 meters and up to 120 meters. We can see the challenges with night-time FR at mid-ranges: without active illumination FR is not possible, while with active illumination FR is possible but a very challenging problem, especially at standoff distances further than 60 meters [7]. . . . . . . . . . . . . . . 24

3.2 The live subject-capture setup using the VIS and mid-range NIR cameras. At the bottom can see a set of face image samples acquired by our system at a night time environment and at variable standoff distances [69]. . . . . . . . . . . . . . 25

3.3 An overview of the designed data collection set-up to collect the indoor and

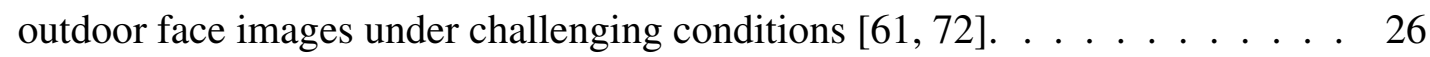

3.4 (a) Side view of camera setup with the 5-position filter wheel positioned in front of the camera. (b) Example diagram illustrating the synchronization of the camera with the filter wheel. . . . . . . . . . . . . . . . . . . . . . . . 28 
$3.5 \quad$ Face images captured using designed SSMW system operating in the SWIR band. . . . 28

3.6 Multi-sensor database collected under the challenging conditions. . . . . . . . . . . . 29

$4.1 \quad$ LBP Descriptors.$\ldots \ldots \ldots \ldots$

4.2 Magnitude of Gabor wavelet transformation. . . . . . . . . . . . . . . 33

4.3 Visualization of HOG Features (right) for input restored image (local shape descriptors). 34

4.4 Convolution $\ldots \ldots \ldots \ldots \ldots \ldots$

$4.5 \quad$ Raw image (top left), only geometrically normalized image (bottom left) and restored images from developed algorithm (right). . . . . . . . . . . . . . 41

4.6 Raw image (top left), geometrically normalized image (bottom left) and photo-metrically normalized images from a selected set of normalization techniques [83] (right). . . . . 41

$4.7 \quad$ Block diagram representation for the selected order of each pre-processing technique. . $\quad 42$

4.8 For $P N$ method applied to raw images (comb1), gallery (VIS-top right) and probe (NIR-bottom right). . . . . . . . . . . . . . . . . . . . . 42

4.9 For $P N$ technique applied to restored images (using the developed image restoration method). . . . . . . . . . . . . . . . . . 43

$5.1 \quad$ Intra-spectral, cross-distance matching scenarios for NIR 30m (Gallery) against NIR 60m (Probe): Performance of baseline face recognition and developed MFSDF sys-

tems for cross-distance images without demographic filtering. Each algorithm was run 4 times and here the rank identification rate is presented for the best performing set. . $\quad 49$

5.2 Intra-spectral, cross-distance matching scenarios for NIR 30m against NIR 90m (Left) and NIR 30m against NIR 120m (Right) without demographic filtering: Each algorithm was run 4 times and here the rank-1 identification accuracy is presented from best set. 50

5.3 Cross-spectral, cross-distance matching scenarios for VIS 1m (Gallery) against NIR $30 m$ (Probe) (Left) and VIS Im (Gallery) against NIR 60m (Probe) (Right). _ . . . . 53

$5.4 \quad$ Box-plots for cross-distance using intra-spectral and cross-spectral matching scenarios after running each experiment 4 times. $\ldots \ldots \ldots$. . . . . . . . . . . . 54

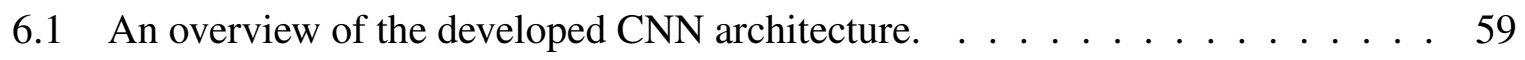


6.2 Selection of Momentum for the Ethnicity and Gender Class. For intra-spectral stratification, visible images were used for training and testing. For cross-spectral stratification, visible images (VIS 1.5m) were used for training (to simulate the most challenging scenario of having a gallery set composed of only visible face images) and NIR images collected at a distance of $30 \mathrm{~m}, 60 \mathrm{~m}, 90 \mathrm{~m}$ and $120 \mathrm{~m}$ distance were used for the

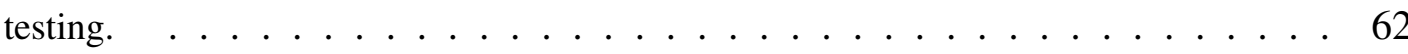

6.3 Demographic filtering results for the ethnicity class. Scenario 1 (Left), is based on overlapping of subjects between training and testing. Scenario 2 (Right), is based on no overlapping of subjects between training and testing. $\ldots \ldots \ldots$. . . . . 63

6.4 Cross-distance matching scenarios for NIR 30m (Gallery) against NIR 120m (Probe):

Performance results when the academic CSU face recognition evaluation tool is used. Each algorithm was run several times and the average rank-1 identification accuracy is presented, i.e. (a) Gender class with Male and Female Group vs. all data without any grouping. (b) Ethnicity class with Asian and Caucasian Group against all data without any grouping (Right). . . . . . . . . . . . . . . 71

6.5 Cross-spectral matching scenarios for Male Data, VIS Im (Gallery) against NIR 30 $m$ (Probe) $($ Left) and VIS 1m (Gallery) against NIR $60 \mathrm{~m}$ (Probe)(Right). . . . . . . 73

6.6 Cross-Spectral matching scenarios for VIS $1.5 m$ (Gallery) against NIR 30m (Probe): Performance of academic CSU face recognition system. Each algorithm was run several times and the rank-1 identification accuracy is presented. Gender class with Male and Female Group vs. all data without any grouping (Left). Ethnicity class with Asian and Caucasian Group against all data without any grouping (Right). . . . . . . . . 76

6.7 Cross-Spectral matching scenarios for VIS (Gallery) against NIR 30m (Probe): Performance of COTS . . . . . . . . . . . . . . . . . . . . 77

6.8 Cross-spectral matching scenarios for VIS $1 \mathrm{~m}$ against NIR 30m (Left) and VIS $1 \mathrm{~m}$ against NIR 60m (Right) with extended gallery set (with demographic filtering). . . . . 79 
7.1 CMC curves comparing the identification performance (first 5 ranks) for cross-spectral matching (Gallery-VIS and Probe-NIR): LBP-CHI (top-left), LBP-DT (top-right), LTP-

CHI (bottom-left) and LTP-DT (bottom-right). Five pre-processing combinations selected such as comb1,comb2,comb3,comb4 and comb5. . . . . . . . . . . . . . . . 86

7.2 CMC curves comparing identification performance (for rank to rank 5) results, for cross-spectral matching, for five selected combinations of pre-processing techniques: LBP/KLDA (top-right), GABOR/KLDA (top-left) and HOG/KLDA (bottom-left). . . 87

7.3 Cross-spectral matching results for Female Stratum: CMC curves comparing the performance for the face images restored from the developed method with raw images. . . 89

7.4 Cross-spectral matching results for Male Cluster: CMC curves comparing the performance of FR system for the face images restored from the developed method with raw images. . . . . . . . . . . . . . . . . . . . 90

$8.1 \quad$ Pipeline for statistical significance tests. . . . . . . . . . . . . . . . . . . 99

8.2 Validation check for parametric test. $\ldots \ldots \ldots$. . . . . . . . . 102

8.3 For Post-hoc test (Left), y-axis represents the three selected groups such as Group 1

(Gabor-Blue), Group 2 (LBP-Green) and Group 3 (HOG-Red) and x-axis represents the rank scores. The association between the groups is calculated using Pearson corre-

lation test (Right). . . . . . . . . . . . . . . . . . 106

8.4 Boxplot for the distance scores when images are matched using different fusion schemes. 108

$8.5 \quad$ Box-plots for the distance scores using combination of pre-processing techniques. . . . 108

9.1 Overview of the developed data filtering network and face recognition system. . . . 113

9.2 An overview of the developed hierarchical classification approach when using SWIR face images captured under various challenging conditions. . . . . . . . 114

9.3 An overview of the developed CNN architecture . . . . . . . . . . . . . 115

9.4 Level 1 (Indoor vs. Outdoor): Classification accuracy results with a selected set of epoch and training sets for CNN. Each boxplot is based on results from 5 randomly selected training and testing sets. . . . . . . . . . . 115

9.5 Level 2 (With vs. No Glass): Classification results. . . . . . . . . . . . . . . 116 
9.6 Level 3 (With vs. Without Illumination): Classification results. . . . . . . . . . 118

9.7 True positive examples from the developed CNN based classification approach.

First row represents the correctly classified images from the level 1 classification into indoors or outdoors class. Second row for the level 2 classification into no glass or with glass. The first two images on the left represent classification for the indoor class into no glass and the other two images for the outdoor class into no glass. On the right side, the first two images represent the indoor class into with glass and the other two images for the outdoor glass into with glass. Third row represents the correctly classified images from the level 3 classification into with and without active illumination. . . . . . . . . . . . . 119

9.8 Misclassified images in level 1 classification. Top row: Indoor class images are falsely labeled as outdoor class. Bottom row: Outdoor images are falsely labeled as indoor. . . . . . . . . . . . . . . . . . . . . . 119

9.9 Face Matching Cross-Scenarios, Intra-spectral: Gallery images in the SWIR band matched against the Probe images in the SWIR band (Top). Cross-spectral: Gallery images in the Visible band matched against the Probe images in the SWIR band (bottom). . . . . . . . . . . . . . . . . . . . . . . . . 121

9.10 Intra-spectral face matching scenarios for SWIR (Gallery) against SWIR (Probe) for with and without grouping of the data into indoors or outdoors. Left: Boxplots for intra-spectral face matching after running each experiment 5 times. Right: Each algorithm was run 5 times and here the rank-1 identification accuracy is presented from the best set. . . . . . . . . . . . . . . . . . 122

9.11 Cross-spectral face matching scenarios for VIS (Gallery) against SWIR (Probe) for with and without grouping of the data into indoors or outdoors. Left: Boxplots for cross-spectral face matching after running each experiment 5 times. Right: The rank-1 identification accuracy is presented from the best set. . . . . 124 
9.12 Cross-spectral (VIS vs. SWIR) face matching results for with and without grouping of the data into indoors and outdoors class into with and without glass. Top: Box-plots for cross-spectral face matching after running each experiment 5 times. Bottom-Left: Each algorithm was run 5 times and here the rank-1 identification accuracy is presented from the best set for All Data (Without Grouping) and Indoor class into Indoor Without Glass (Indoor-WTOG) or Indoor With Glass (Indoor-WTG). Bottom-Right: For All Data and Outdoor class into Outdoor Without Glass (Outdoor-WTOG) or Outdoor With Glass (Indoor-WTG). . . . . . . . . . . . . . . . . . . 126

9.13 Cross-spectral (VIS vs. SWIR) face matching results for with and without grouping of the data into indoors and outdoors class into with and without active illuminator. Top: Box-plots for cross-spectral face matching after running each experiment 5 times. Bottom-Left: Each algorithm was run 5 times and here the rank-1 identification accuracy is presented from the best set for All Data (Without Grouping) and Indoor class into Indoor Without Illuminator (Indoor-WTOIL) or Indoor With Illuminator (Indoor-WTIL). Bottom-Right: For All Data and Outdoor class into Outdoor Without Illuminator (OutdoorWTOIL) or Outdoor With Illuminator (Outdoor-WTIL). . . . . . . . . . . . 127

9.14 Overview of the developed fusion scheme that performs wavelength-based classification. (b) Feature extraction is performed based on methods presented in the bottom figure at left side and (c) classification is performed based on methods presented in bottom figure at right side [3]. . . . . . . . . . . . . . . . . . . . . . . 129

9.15 An overview of the developed CNN architecture . . . . . . . . . . . . . . 130

9.16 Training and Testing Data for CNN Architecture for Classification. . . . . . . . 131

9.17 Classification accuracy results with a selected set of epoch for CNN. Each boxplot is based on results from 5 randomly selected training and testing sets. . . . . . . . . . . 132

9.18 Classification accuracy results for Set 2: Scenario 2 and Scenario 3 with selected set of training sets for CNN. Box-plots are based on results from 4 randomly selected training and testing sets. 
9.19 Flow chart diagram representing the process involved in the developed approach for classifying the frontal and non-frontal face images [3]. . . . . . . . . . . . . . 135

9.20 Cross-Spectral Face Matching: Gallery images in the VIS band (Left) are matched against the Probe images in the SWIR band captured from the designed SSMW system at five wavelengths of $1150,1250,1350,1450$ and $1550 \mathrm{~nm}$ (Right). . . . . . . . . 136

9.21 Cross-spectral face matching scenarios for VIS (Gallery) against SWIR (Probe)

for with and without grouping of the data into individual wavelengths. The rank-1 identification accuracy is presented from the best set. . . . . . . . . . 137

9.22 Cross-spectral face matching scenarios for VIS (Gallery) against SWIR 1350 $\mathrm{nm}$ (Probe) for the data into individual wavelengths. Comparison for individual vs. Fusion based matchers. . . . . . . . . . . . . . . . . . . . . . 137

9.23 Cross-spectral face matching scenarios for VIS (Gallery) against SWIR (Probe) for with and without grouping of the data into individual wavelengths. Boxplots for cross-spectral face matching after running each experiment 5 times.

9.24 Cross-spectral face matching scenarios for VIS (Gallery) against SWIR 1550 $\mathrm{nm}$ (Probe) for the data grouped into individual wavelengths. Comparison of individual vs. Fusion based matchers. . . . . . . . . . . . . . . . . . . . . . . 139

10.1 An overview of the developed hierarchical classification approach for the face images captured from the mobile phones under various conditions. Please note that after performing the first three steps (video into frames, face detection and pose estimation), the frontal face images were selected to perform the classification starting from top (Level 1) to bottom $($ Level 3). [23] . . . . . . . . . . . . . . . . . . . . . . 143

10.2 Developed CNN scheme to perform hierarchical classification. In CNN architecture: $\mathrm{C}$ represents the convolution layer, $\mathrm{P}$ the pooling layer, $\mathrm{R}$ the rectification layer and $\mathrm{FC}$ for the fully connected layer. The number represents the layer number for example, C1 represents the first convolution layer. CNN architecture consists of three convolution layers (C1, C2 and C3), two pooling layers (P1 and P2), one rectified layer (R1) and two fully connected layers $(\mathrm{FC} 1$ and $\mathrm{FC} 2)$. 
10.3 Classification accuracy vs. Epoch after running a set of five experiments. . . . . . . . 147

10.4 Classification accuracy results with a selected set of epoch and training sets for CNN.

Each boxplot is based on results from 5 randomly selected training and testing sets. . . 148

10.5 Classification results for Level 3 classification for all the phones. . . . . . . . . . 150

10.6 Face detection for the face images captured with the mobile phones under un-constrained conditions. . . . . . . . . . . . . . . . . . . . . 152

10.7 Viola Jones detector with True Positive, False Positive and False Negative results. . . . 153

10.8 Pose estimation from $-90^{\circ}$ to $+90^{\circ}$ angle for the detected face images. . . . . . . 154 


\section{List of Tables}

$2.1 \quad$ Previous work related to heterogeneous face matching. . . . . . . . . . . . . 15

3.1 Summary of face image databases for cross-spectral and cross-distance matching using long range data. Note: WVU database consists of video sequences for each subject. Still images were selected from those sequences to perform the FR studies. In this work $103 \times 4$ NIR outdoors (2 for gallery and 2 for probes) and 103 visible indoors face images per subject were used. . . . . . . . . . . . 25

3.2 Database distribution (number of images) for each level. . . . . . . . . . . . 27

5.1 Summary of total number of face recognition experiments are performed for each dataset, with and without demographic filtering. To perform the FR experiments, two FR cross-scenarios are selected cross-distance (CD) and crossspectral (CS). . . . . . . . . . . . . . . . . . 46 
5.2 In this table the intra-spectral, intra-distance matching scenarios without demographic filtering for the database collected from 103 subjects are investigated. Experimental results are presented while running all CSU FR algorithms when using $20 \%, 40 \%$ and $60 \%$ of the NIR data for training and the rest data $(80 \%, 60 \%$ and $40 \%$ respectively) for testing. When CSU is used, the experiments were run 10 times and the identification rank-1 accuracy presented here are the means. G8 is also tested for each scenario. Note that there is one case $(90 \mathrm{~m})$ where the identification rank-1 accuracy was about $38 \%$ vs. $59 \%$ with the $120 \mathrm{~m}$ data. This was because the experiment was run once and it was noticed that some $90 \mathrm{~m}$ face images selected for matching were more heavily affected by background noise (e.g. passing cars and illumination) due to the time of the collection. There is no overlap of subjects between training and testing. . . . . . . . . . . . . . . . . . . . 47

5.3 In this table the intra-spectral, cross-distance matching scenarios (without demographic filtering) are investigated. Experimental results are presented when running all FR algorithms using 50\% of the NIR data for training and the rest of the data for testing (database consists of 103 subjects). For each algorithm including, AFIES, COTS and the developed MFSDF, the experiments were run 4 times and the rank-1 identification accuracy is presented here. There is no overlap of subjects between training and testing. . . . . . . . . . . . . . . 48

5.4 In this table the cross-spectral, cross-distance matching scenarios (without demographic filtering) are investigated. Experimental results are presented when using $50 \%$ of the data for training and the rest data for testing. There is no overlap of subjects between training and testing. Experiments are run 4 times and here the rank-1 identification accuracy is presented. . . . . . . . . . . 51 
6.1 Summary of stratification (demographic filtering) results for the ethnicity class based on CNN for each dataset. To perform CNN, the model is trained for the challenging testing database. WVU database consists of 103 subjects and LDHF database consists of 100 subjects. Scenario 1, WVU (50\%) database for training and the rest of the database for testing. Scenario 2, LDHF (ALL) and WVU (50\%) database for training and the rest of the database for testing. . . . 64

6.2 Summary of stratification results for the Ethnicity class based on CNN for each dataset. To perform stratification, model is trained on 9 different databases. Scenario 1, 9 databases for training and WVU database for testing. Scenario 2, 9 other databases and WVU 25\% database for the training and the rest of the WVU database for testing. . . . . . . . . . . . . . . . . . . 65

6.3 Summary of demographic filtering results for the gender class based on CNN for each dataset. To perform CNN, model is trained for the challenging testing database. Scenario 1, where NIR images are used for training. For Scenario 2, VIS images are used for training. In both the scenarios, LDHF database is used for the training and WVU database is used for testing. Scenario 3, the VIS band face images from both the LDHF and WVU databases are used for training, while the WVU database for testing. . . . . . . . . . . . . 66

6.4 Summary of demographic filtering results for the ethnicity and gender class based on CNN for each dataset. To perform CNN, the model is trained for the challenging testing database.$\ldots \ldots \ldots$. . . . . . . . . . . 67

6.5 Summary of total number of FR experiments performed, with and without grouping of data in terms of ethnicity and gender class. To perform the FR experiments, two FR cross-scenarios are selected cross-distance (CD) and crossspectral $(\mathrm{CS}) . \ldots \ldots \ldots \ldots \ldots \ldots$ 
6.6 The intra-spectral, cross-distance matching scenarios for Male Class are investigated. The experimental results when running all FR algorithms using $50 \%$ of the NIR data for training and rest of the data for testing (No overlap of subjects) are presented. For each algorithm including, AFIES, COTS and developed MFSDF, the experiments were run 4 times and the rank-1 average identification accuracy is presented. . . . . . . . . . . . . . . . . 70

6.7 The intra-spectral, cross-distance matching scenarios for with and without grouping of data in terms of ethnicity class are compared in this table. The experimental results when running all CSU FR algorithms and using 50\% of the NIR data for training and the rest of the data for testing (No overlap of subjects) are presented. The experiments were performed 4 times and the rank-1 average identification accuracy is presented here. . . . . . . . . . . . . 72

6.8 The cross-spectral, cross-distance matching scenarios are investigated. The experimental results when using 50\% of the data for training and the rest data for testing are presented. Experiments were performed 4 times and the rank-1 average identification accuracy is presented. . . . . . . . . . . . . . . . . 74

6.9 The cross-spectral, cross-distance matching scenarios for with and without grouping of data in terms of ethnicity class are compared. The experimental results when running all CSU FR algorithms using 50\% of the NIR data for training and the rest of the data for testing are presented. The experiments were performed 4 times and the rank-1 average identification accuracy is presented. COTS was also tested here $\ldots \ldots \ldots \ldots$. . . . . . . . . . . . . 76

6.10 In this table investigate the intra-spectral, cross-distance matching scenarios with and without demographic filtering, with extended gallery: experimental results when running all FR algorithms using LDHF data for training and our data for testing. . . . . . . . . . . . . . . . . . . 78

6.11 Measure of statistics (mean, median, minimum and maximum) for attained image quality scores: experimental results when visible images are used as reference images and long distance images (NIR 60m) as query images. . . . . 79 
7.1 Cross-spectral Matching Results (Gallery in VIS (1m-indoor) under controlled conditions and Probe images in NIR): Rank identification accuracies for probe images at 4 different distances in nighttime environment: $30 m, 60 m, 90 m$ and $120 m$ distance. . . 91

7.2 Cross-spectral matching results for Soft Biometric Traits (Gallery in VIS (1 $m$-indoor) under controlled conditions and Probe images in NIR): Rank identification accuracy for probe images at 4 different distances in nighttime environment: $30 m, 60 m, 90 m$ and $120 m$ distance $\ldots \ldots \ldots \ldots \ldots$. . . . . . . . . . . . . . 92

7.3 In this table investigate the cross-spectral, cross-distance matching scenarios \begin{tabular}{|c|c|c|}
\hline for database before and after the usage of developed image restoration and de- \\
\hline
\end{tabular} mographic filtering of male class into with or without beard and female class into Asian and Caucasian class: Experiments were run several times and the rank-1 identification accuracy presented here (average). Developed-Set 1: Only Developed Fusion Scheme applied to the images, Developed-Set 2: Both developed image restoration and fusion scheme applied to the images. For Set 2 with Male and Female Class further sub-clustering is performed: MWB, MWOB, $\mathrm{FA}$ and $\mathrm{FC}$ class $\ldots \ldots \ldots \ldots . \ldots \ldots$

8.1 Correlation coefficient when Pearson test is used for match scores for Group 1 (Gabor), Group 2 (LBP) and Group 3 (HOG) for comb2. . . . . . . . . . . . . . . . . 101

8.2 The distribution of match scores for features extracted using the Gabor descriptor when the second combination of pre-processing algorithms is used - see Fig. $\mid 4.7$. $\ldots$. . . . . 102

8.3 Homogeneity of variance using Levene test for match scores for Group 1 (Gabor), Group 2 (LBP) and Group 3 (HOG) for comb2. . . . . . . . . . . . . . . . . 103

8.4 The match scores are converted into rank scores for each probe subject to perform the Friedman test. Here, the rank scores are represented for the first 9 samples (in total $6400 \times 3$ ), where rows represent rank scores for the first probe image when compared against the first 9 gallery images and columns represent the feature descriptors. . . . . 104

8.5 Friedman and Post-hoc tests for match scores to find out the statistical significant difference between groups. . . . . . . . . . . . . . . . . . 105

$8.6 \quad$ Number of outliers and location of these outliers from box-plots for comb5. . . . . . . 107 
8.7 Selection of Pre-processing combination (for the face images at $30 \mathrm{~m}$ ) based on results from box-plots. Performance is affected by median, standard deviation, number of outliers and location of these outliers. . . . . . . . . . . . . . . . . . . . 109

8.8 Selection of pre-processing combination (for stratified database) based on results from box-plots. Performance is affected by median, standard deviation, number of outliers and location of these outliers. . . . . . . . . . . . . . . . . . . . . . 110

8.9 Rank-1 identification accuracy results for ALL Data without any image restoration, fusion and stratification. . . . . . . . . . . . . . . . . . 111

9.1 Hierarchical classification results from CNN: randomly selected training sets. . 120

9.2 In this table the results for the intra-spectral, cross-spectral matching scenarios for with and without grouping of the data are presented. Intra-spectral matching scenario for SWIR (Gallery) against SWIR (Probe) under unconstrained conditions. Cross-spectral matching scenario for VIS (Gallery) against SWIR (Probe) under unconstrained conditions. NIR 60m (Probe) . . . . . . . . . . . 123

9.3 Classification results based on developed $\mathrm{CNN}$ architecture. . . . . . . . . . 133

9.4 The Cross-spectral matching scenarios for VIS 1.5m (Gallery) against SWIR $(1150,1250,1350,1450$ and $1550 \mathrm{~nm})$ are performed. The experimental results are presented when the developed FR algorithms is run using 50\% of the data for training and the rest of the data for testing (with no subject overlap). The experiments were run 5 times and the rank-1 identification accuracy is presented here are as average. . . . . . . . . . . . . . . . . . 138

10.1 Classification results from CNN: Phone Type. DB1 consists of 100 subjects and DB2 consists of 80 subjects. . . . . . . . . . . . . . . 146

10.2 The best classification results from CNN for all the phones: Indoor vs. Outdoor. 148

10.3 Classification results from CNN: Indoor vs. Outdoor . . . . . . . . . . . . 149

10.4 Classification accuracy results from CNN (\%): Close (1m) vs. Far (10m) distance. 150

10.5 Highest classification accuracy results from CNN: Close (1m) vs. Far (10m) distance. . . . . . . . . . . . . . . . . . . . . 151 
10.6 Face matching for with and without grouping of data. . . . . . . . . . 154

10.7 Impact on Face Matching Accuracy when either of Data Grouping or Databased Pre-Screening was used $\ldots \ldots \ldots$. . . . . . . . . . . . . . 155

10.8 Classification results for extended database (100 subjects) from CNN for all the phones: Indoor vs. Outdoor. ․ . . . . . . . . . . . . . . . . . 155

10.9 Impact on Face Matching Accuracy when Data Grouping or Data based PreScreening was used for extended database (100 subjects). . . . . . . . . . . . . 156 


\section{Chapter 1}

\section{Introduction}

\subsection{Face Recognition Systems}

Biometric systems based on unique physiological and behavioral characteristics offer a natural and reliable solution for the successful identification of individuals in law enforcement and security applications [1]. Traditional token-based (passport, driver's license, ID card) and knowledge based systems (passwords or personal identification number) can be easily manipulated, hacked or shared, therefore compromising system security. Whereas, biometric traits (such as fingerprints, face, iris, retina, voice etc.) offer a unique, secure, quicker and highly accurate identification system [1, 2].

Face is considered among the top choices because, unlike other modalities, it is easy to capture even in a non-cooperative way at a distance, it is non-invasive and the face-based recognition technology is fairly accurate [3]. While there is a plethora of FR applications, the most common include video surveillance, authentication for secure banking, financial transactions, access control to computer systems and government-buildings and traveler's screening (at airports, train stations, etc.).

FR system can operate either in face verification (authentication) or face identification mode [2]. Face verification is represented as one to one matching, with the task of validating a person's claimed identity by comparing the captured probe image against the template image (gallery) [2]. Identification task is related to one-to-many matches, and identification is per- 
formed by comparing the probe face image against all the template images in the database.

FR systems generally consist of five modules; image acquisition, face/eye detection, face alignment, feature extraction and matching (see Fig. 1.1). After pre-processing, feature extraction is performed before matching, which is based on the computation of similarity scores when comparing query and gallery images.

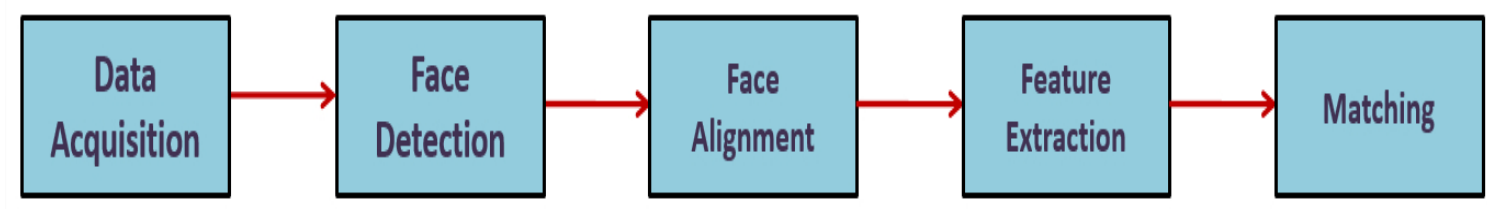

Figure 1.1: Block diagram representation of a typical face recognition system.

There is a variety of face recognition algorithms discussed in the literature including; appearance-based, model-based and texture-based ones. The appearance-based techniques are based on the entire face region. Principal component analysis (PCA) [4] or linear discriminate analysis (LDA) [5] are the most well-known examples of such techniques. Model based techniques such as elastic bunch graph matching is based on a set of facial landmark points such as corners of eyes, tip of the nose and corners of the mouth. These landmark points are sometimes referred to as fiducial points. Local binary patterns (LBP) and Local Ternary Patterns (LTP) descriptors are considered as the most common methods to get the texture information.

\subsection{Challenges for Face Recognition}

The existing standard face recognition systems result in operationally acceptable identification rates when performing the FR under highly controlled conditions; the light source and direction, face pose, standoff distance (i.e. the distance between the camera and the face), as well as the background, for example, are kept within tightly controlled limits [6, 7]. However, in law enforcement and security applications, operators deal with mixed FR scenarios that involve matching face images captured by:

- Different Cameras Sensors: When the probe and gallery face images are captured from different camera sensors. For example, the gallery images are captured using visible 


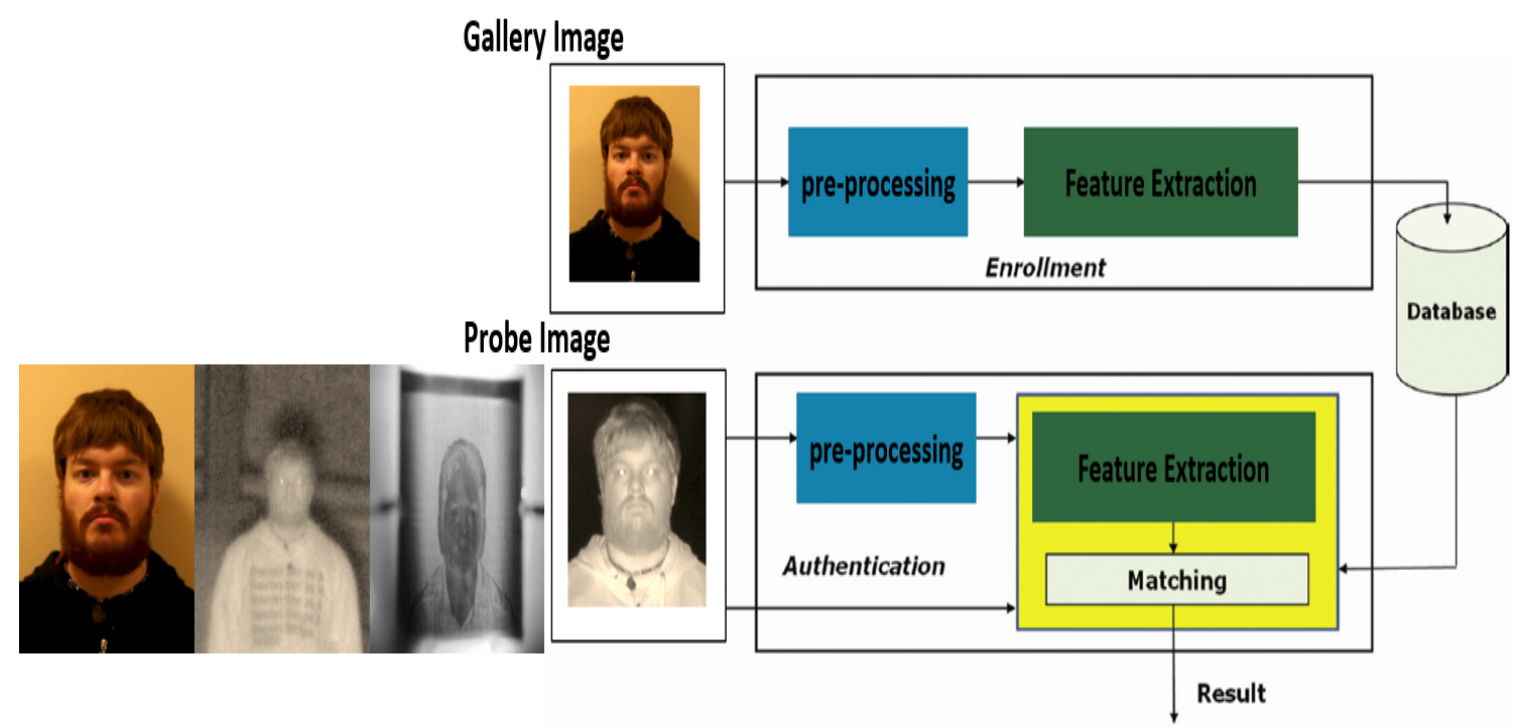

Figure 1.2: Mixed face recognition scenarios involve matching probe images captured by any camera sensor, standoff distance and illumination conditions.

camera under fully controlled conditions. Whereas, the probe images are captured using NIR and SWIR cameras as shown in Fig. 1.2. The difference in appearance between the images sensed in the visible and infrared band is due to the properties of sensors.

- Night-Time Environments: The quality of the image degrades when the face images are acquired outdoors in night-time conditions.

- Long Standoff Distances: When the subject is far away from the camera (See in Fig. 1.3 against the good quality face images (e.g. mug shots) acquired using high definition camera sensors (e.g. DSLR cameras) is responsible for performance degradation of conventional face recognition systems.

- With Tinted Glass: When the face images are captured through the tinted glass panels.

Under such challenging conditions, the traditional face recognition systems such as based on Local Binary Patterns or Linear Discriminant Analysis often provide unsatisfactory results. We need to develop an efficient and reliable system which is capable of face based evidence to be extremely accurate so that it can provide to law enforcement officers a solid lead, to either identify or rule out suspects. 


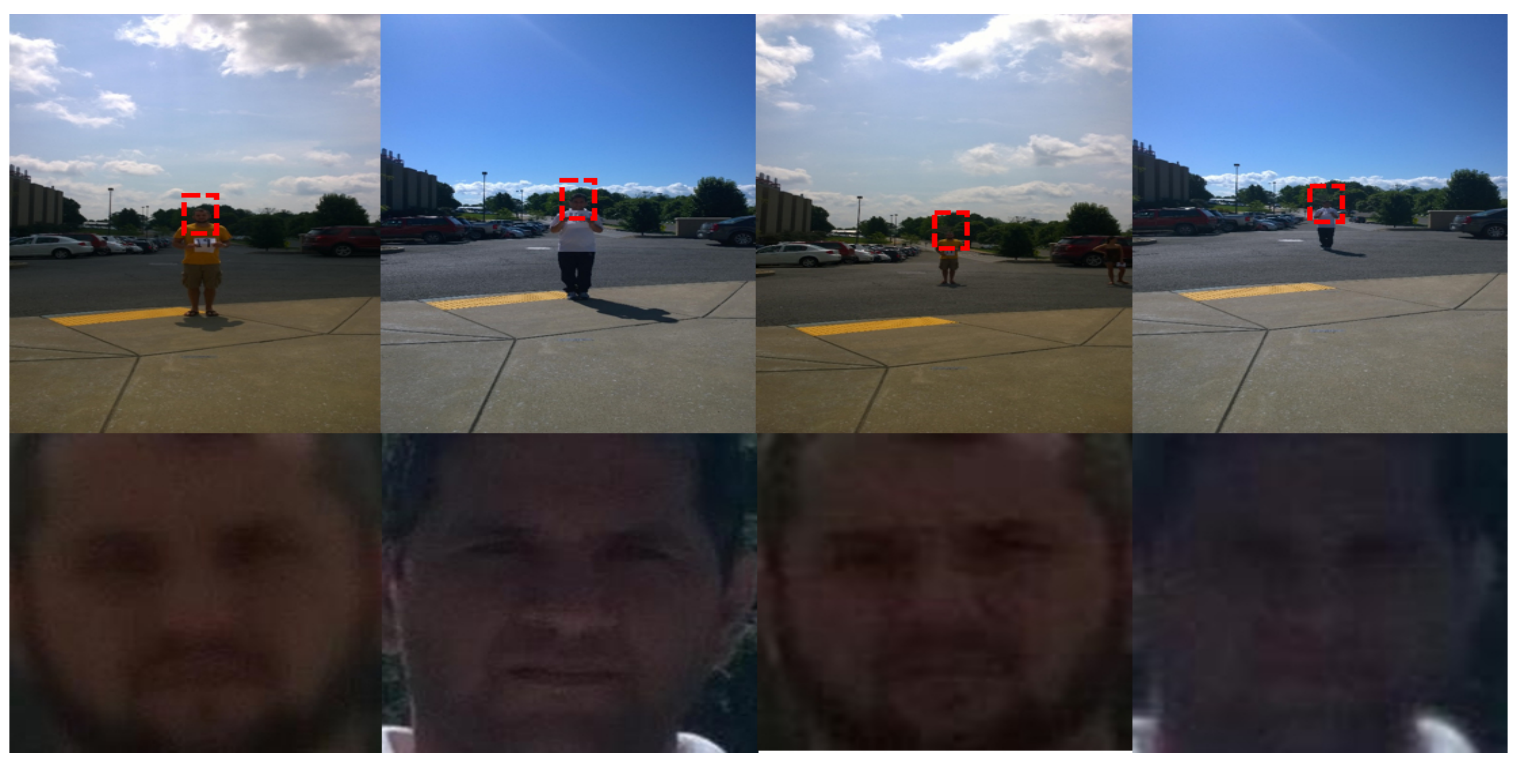

Figure 1.3: Face images collected at standoff distance of $5 \mathrm{~m}$ and $10 \mathrm{~m}$.

\subsubsection{General Research Challenges}

When the data collection is performed using sensors that operate at different spectral bands (UV, visible, infrared, and thermal), the main questions that need to be answered are:

- Either independently or in combination with others, when designing such a system under either controlled or uncontrolled conditions which spectral band should be selected to solve the problems of human recognition? To be able to answer this question we need to go beyond the visible band and to the infrared (IR) spectrum [8].

- How do we design a heterogeneous face recognition system that can recognize people for the face images captured in night-time environment, outdoors and at long standoff distances?

\subsubsection{Focused Research Challenges}

- Which feature descriptor can result in better identification performance?

- Why do we need fusion and which fusion scheme can result in better performance?

- What is the impact of data filtering in terms of gender, ethnicity, indoors, outdoors?

- Which image restoration method can restore the highest quality images? 
- How we can support our experimental results?

\subsection{Research Objective}

The main objective of this research work is to design and develop a night vision, efficient face recognition/matching system that can provide solutions on such operational scenarios in order to improve system performance, i.e. rank-1 identification rate.

\subsubsection{Contributions}

In order to deal with the problem of identifying people in both at day and night-time environments, at variable distances and illumination conditions, various types of visible (e.g. with telephoto lenses) and infrared sensors (Near, Short-Wave, Mid-Wave, and Long-Wave IR), under either single-spectral or multi-spectral recognition settings are required [9, 10]. We also know that there are certain settings and configurations that provide increased identification performance during night-time operations when using passive IR sensors [9], active IR sensors [11] or visible band sensors that operate during day-time and at medium distances [12].

To address the problem of recognizing people when the face images are collected in nighttime environment and at long standoff distance, NIR imaging system that has the capability of delivering detailed surveillance assessment imagery at any outdoor illumination conditions (either daylight, low-light or no-light conditions) is designed. Due to its optical capabilities, this camera system enables detailed human assessment in complete darkness at long ranges (beyond 400 feet) as shown in Fig. 1.4. However, in practice, even though the system operator can read text, numbers, and detect humans at long ranges, the system is not designed to have the capability to automatically and efficiently recognize humans via their face images.

To address the problem of images captured through tinted glass, imaging systems in the SWIR band play an important role. It is a part of the reflected IR (active) band (in these experiments, it ranges from $0.9 \mu \mathrm{m}$ to $1.7 \mu \mathrm{m}$ ). In harsh environmental conditions characterized by unfavorable lighting and pronounced shadows, FR based on SWIR images may be advantageous [13-16]. SWIR imagery is more tolerant to low levels of obscurants like fog and smoke 


\section{Controlled, Cooperative User Scenarios (No Tagging)}
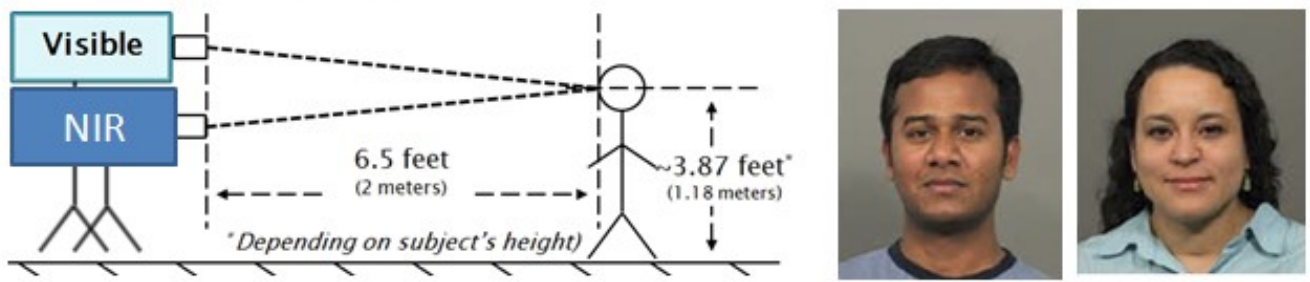

\section{Long Range, Uncontrolled User Scenarios (No Tagging)}

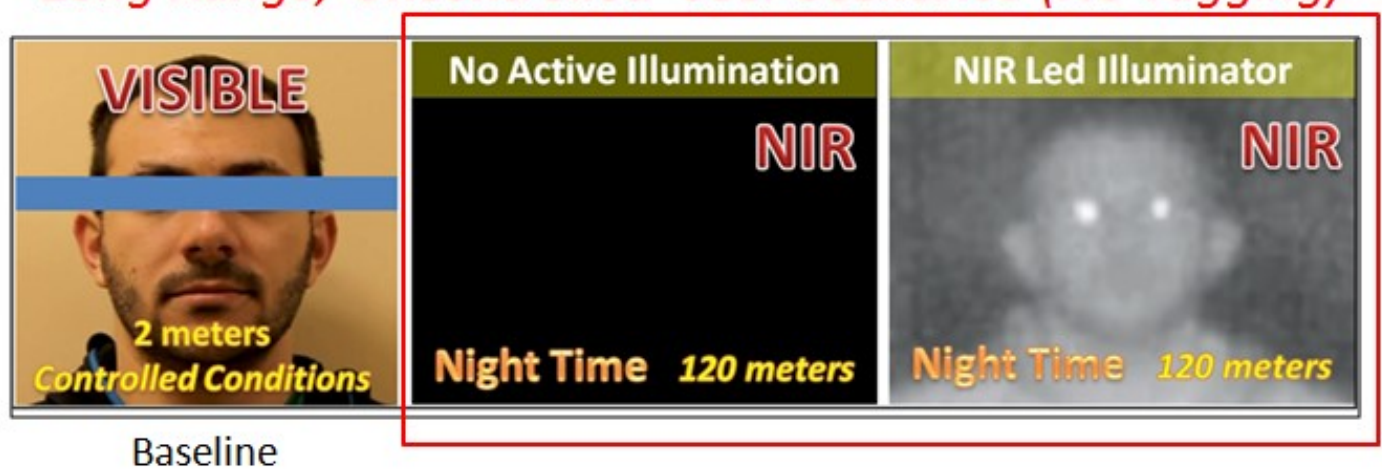

Figure 1.4: Data collection scenarios of both visible and infrared face data, under controlled (top image) or uncontrolled (bottom image) conditions [7].

[3, 17, 18]. The main benefit is that, it can take advantage of sunlight, moonlight, or starlight [14, 19, 20]. It offers the possibility to capture the images through tinted glass as shown in Fig. $1.5[21]$.

This work is an effort to investigate some of the challenges of face recognition by focusing on four main directions (see Fig. 1.6):

(i) To conduct this study, four different sets of challenging databases are collected under

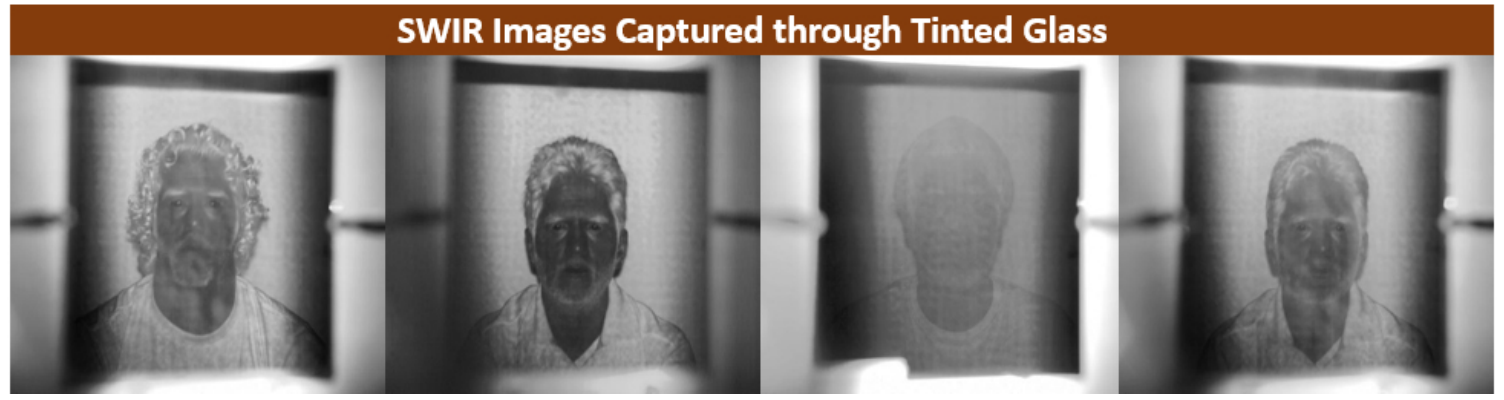

Figure 1.5: Probe face images are captured in the SWIR band through tinted glass.

controlled and uncontrolled conditions in our lab. The face images are acquired, at day-time, night-time conditions, indoors or outdoors and at different standoff distances, up to more than 
100 meters away using visible, NIR and SWIR camera sensors.

(ii) Developed a multi-feature scenario depended fusion (MFSDF) scheme that can assist in improving FR performance at the aforementioned challenging matching conditions.

(iii) Developed convolutional neural network (CNN) based, scenario-dependent and sensor adaptable hierarchical classification frameworks in visible, NIR and SWIR band. The developed network is designed to automatically categorize face data captured under various challenging conditions, before the FR algorithms (pre-processing, feature extraction and matching) are used. The impact of data grouping in terms of demographic information about the user gender, ethnicity and images in terms of indoors, outdoors is explored for the purpose of improving cross-spectral face recognition (FR) performance.

(iv) One of the major issues is that the image quality degrades as a function of the standoff distance and therefore, either same-spectral or cross-spectral matching becomes a very challenging process. An image restoration method to restore the good quality images is developed.

\subsubsection{Designed and Developed Heterogeneous Imaging System}

NIR Imaging System: In this work, a dual band database in the VIS (baseline) and NIR spectrum of 103 subjects at night-time environments and variable long standoff distances, starting from $30 \mathrm{~m}$ and up to $120 \mathrm{~m}$, in $30 \mathrm{~m}$ intervals is collected and used to demonstrate the challenges associated with the problem. Then, a set of experiments were performed in order to demonstrate the possibility for long range face recognition, at night and in the NIR band.

Multi-Scenario (MS) SWIR Imaging System: Face images were captured in SWIR band under multiple scenarios, including, face data captured indoors, outdoors, with or without tinted glass panels $(0 \%, 80 \%$ and Solar) placed in front of a subject's face or even with active illumination at $1550 \mathrm{~nm}$.

Single Sensor Multi-wavelength (SSMW) Imaging System: SWIR face database was captured using single sensor multi-wavelength system, including, face data captured at five different SWIR wavelengths ranging from $1150 \mathrm{~nm}$ and up to $1550 \mathrm{~nm}$ in increments of $100 \mathrm{~nm}$.

Multi-Sensor Imaging System: A unique multi-sensor database (using Samsung S4 Zoom, Nokia 1020, iPhone 5S and Samsung S5) was collected containing face images indoors, out- 


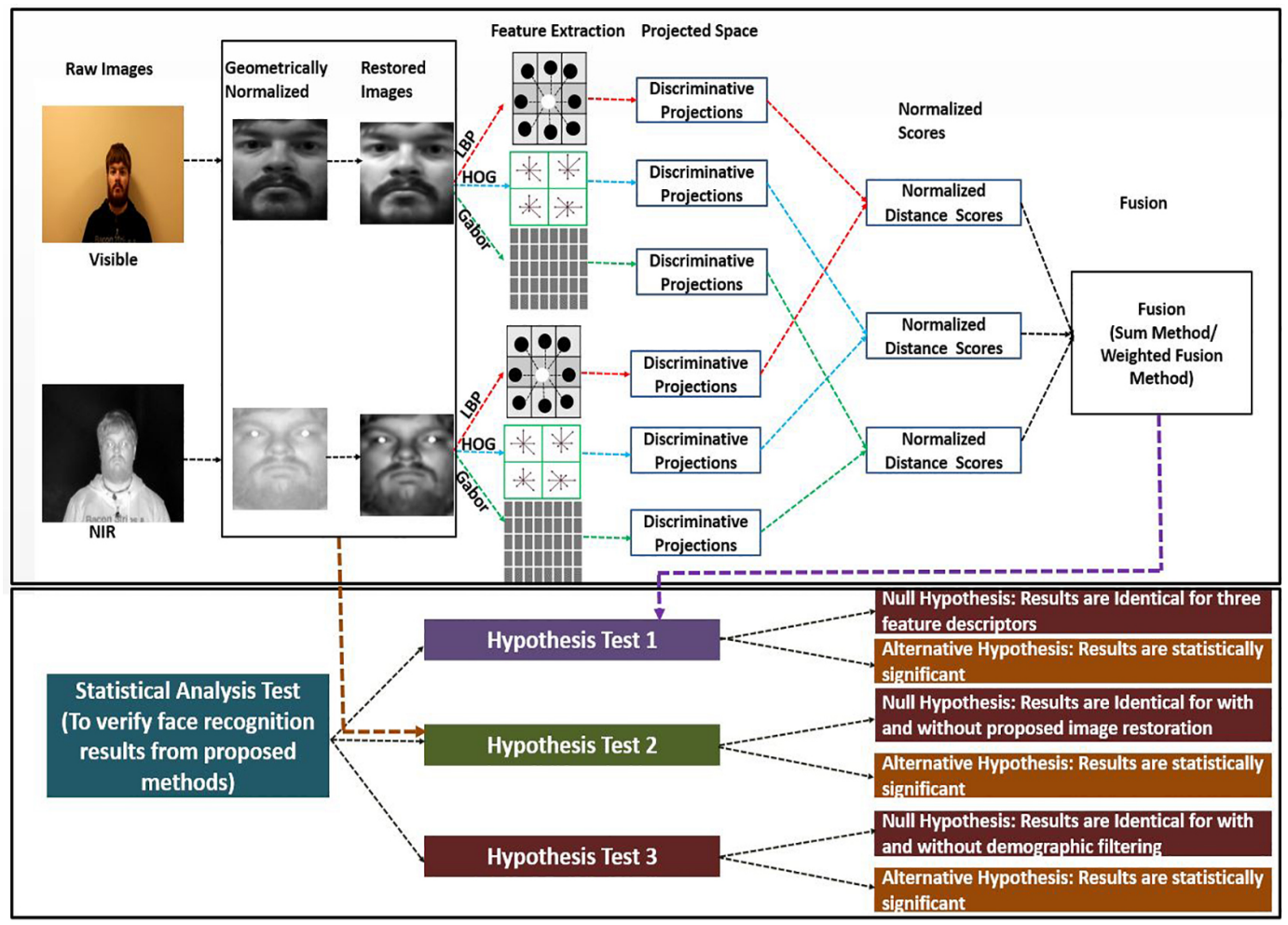

Figure 1.6: Overview of conventional face recognition system using developed pipeline (top box) and incorporation of statistical tests for the verification purpose.

doors, with yaw angle from $-90^{\circ}$ to $+90^{\circ}$ and at two different distances of $1 \mathrm{~m}$ and $10 \mathrm{~m}$.

First a baseline using commercial and academic face matchers was established. The rank1 identification results for inter-distance, intra spectral, cross-distance and cross-spectral face recognition were reported.

\subsubsection{Study 1: Developed Multi-feature Scenario Depended Fusion Scheme}

First, Gabor Wavelets, Histogram of gradients (HOG) and Local binary patterns (LBP) feature descriptors are empirically selected and, then, a set of fusion score level schemes are developed to improve the face recognition performance.

\subsubsection{Study 2: Developed Deep Features Based Data Filtering Network}

Developed a scenario dependent and sensor adaptable convolutional neural network (CNN) to perform the data filtering. While the manual filtering can be done, such a process is time consuming, especially when dealing with large datasets [22, 23]. Therefore an automated way of classifying images into specific scenario is needed. If data organization is performed the 
FR system can be efficient in terms of both time and potential errors resulting from manual labeling.

Demographic Filtering in NIR Band: The usage of demographic filtering of data (ethnicity, gender and facial hair class), before face matching for the purpose of improving face recognition performance is developed. One of the main examples is discussed in [1], where one of the state-of-the-art FR commercial software was assisted by non-facial context data to work efficiently. Developed algorithmic approach for the demographic filtering of soft biometric traits in terms of male class into male with or without beard and female class into Asian or Caucasian class. It is working well for both visible and NIR face images. The demographic data is further used to perform the face recognition experiments.

Data Filtering in SWIR Band: Two databases are selected in SWIR band including multiscenario and multi-wavelength database.

Multi-Scenario Database: CNN based, multi-scenario framework is designed, where hierarchical data filtering is performed as follows: Level 1 , face images are classified into Indoor or Outdoor; Level 2, face images are further classified into those captured behind various types of tinted glass or not; and, finally, Level 3, face images are classified into those captured when active illumination was used or not.

Multi-wavelength Database: CNN framework categorizes face data into individual wavelength into $1150,1250,1350,1450$ and $1550 \mathrm{~nm}$.

Data Filtering for Multi-Sensor Database: Developed CNN framework is used where trilevel hierarchical classification is performed as follows: Level 1, face images are classified based on phone type; Level 2, face images are further classified into indoor and outdoor images; and, finally, Level 3, face images are classified into close (1m) and far, low quality, (10m) distance.

\subsubsection{Study 3: Developed Image Restoration Scheme}

In practical applications, one of the problems with human face-based recognition is that it is often the case where the training material (i.e. the gallery dataset of face images, normally captured in a controlled environment of a set of individuals enrolled in the dataset) is only avail- 
able in the visible spectrum (see Fig. 1.6). Thus, one needs to perform the very challenging task of matching cross-scenario face images. One way of attempting to deal with such a challenge is through photometric normalization [24], utilizing algorithms such as contrast limited adaptive histogram equalization $(C L A H E)$ [25]. While such algorithms often are shown to improve identification performance, their capability in practical applications is limited due to the fact that face images of suspects (probes), available from surveillance data, are of low quality. The reasons are that such face images are captured using low quality sensors at challenging conditions (including variable distances, off angle, and during night-time) while also they need to be successfully cross-matched to the available mug shots (i.e. good quality face images (gallery), typically captured in the visible band). To address this problem, an image restoration method to improve the quality of distorted, long range NIR face images is developed.

\subsubsection{Study 4: Statistical Hypothesis Testing}

Statistical hypothesis tests are performed to find the statistical significance of incorporating developed image restoration, score level fusion schemes, as well as the demographic filtering in terms of gender, ethnicity, and facial hair. The conducted set of statistical tests are represented by a pipeline which consists of, selection of statistical test for our database, hypothesis test 1 , hypothesis test 2 and hypothesis test 3 as shown in Fig. 1.6.

Both commercial and academic face matchers are used and a set of experiments is performed, indicating that developed image restoration, fusion schemes and the usage of demographic information of the database, achieved significantly better performance results e.g. the developed fusion scheme results in 54 percent point (pp) higher recognition performance than the baseline face matchers (established using a commercial and a set of academic) and can be utilized to improve cross-spectral matching performance on diverse scenarios.

To the best of our knowledge, this is the first-time deep learning based network for the grouping of the data (collected under un-constrained conditions) is developed. The data filtering is performed in terms of:

- Demographic information (Female Asian, Female Caucasian, Male With Beard and Male Without Beard stratum) for the face images collected in night-time, at long range distance 
and in the NIR band is developed. Also, there is no reported work that evaluates the impact of grouping soft biometrics (for the purpose of data filtering) in terms of ethnicity, gender and facial hair in a cross-spectral FR system (NIR images collected in night-time and at long range distance).

- Image filtering into indoors, outdoors, with and without tinted glass and into wavelength in SWIR band.

- Image filtering into sensor type, indoors and outdoors in VIS band.

\subsection{Thesis Organization}

In this section, an outline of the rest of the dissertation will be described.

- Chapter 2 describes existing work related to the heterogeneous face recognition systems in the infrared band. In this chapter, the developed face recognition system in heterogeneous environments is discussed. Also, the approaches developed by researchers for data filtering and image restoration are discussed.

- Chapter 3 describes the design and development of heterogeneous imaging system and methods developed to optimize the set up to acquire the good quality images. An overview of the long distance night-time database collected in NIR band, multi-wavelength database collected in SWIR band is provided. Finally, the multi-sensor database collected using 4 different cell phone devices under challenging conditions is discussed.

- Chapter 4 presents the developed algorithms for face matching based on multi-feature score level fusion scheme, automatic data filtering based on deep learning network and image restoration method to improve the quality of distorted images collected at long distances.

- Chapter 5 presents the evaluation of developed multi-feature based face matching system and results from both commercial and academic face matchers. 
- Chapter 6 presents the results and evaluation of the developed convolution network architecture for the automatic demographic filtering of the data in terms of ethnicity, gender and, facial hairs class.

- Chapter 7 presents the identification results based on the developed image restoration method.

- Chapter 8 presents the statistical significance of incorporating developed algorithms using statistical analysis tests.

- Chapter 9 presents the evaluation of $\mathrm{CNN}$ based data filtering network and fusion based face matching scheme on short wavelength infrared database collected under un-constrained conditions.

- Chapter 10 presents the evaluation of CNN based data filtering network and fusion based face matching scheme on multi-sensor database collected indoors, outdoors, at variable standoff distances.

- Chapter 11 finally summarizes overall conclusions drawn and the main contributions of the dissertation, as well as suggested future work. 


\section{Chapter 2}

\section{Literature Review}

In this chapter, the existing systems for the heterogeneous face matching when the database is collected in un-constrained environment conditions are discussed. Then, the challenges related to existing systems and the developed system to address those challenges are discussed.

\subsection{Heterogeneous Face Matching}

\subsubsection{Reported Work}

- Synthesis Based Infrared Face Matching: Liu et al. [26] developed a method for synthesizing VIS images from NIR images. This method is based on linear mapping between the images collected from two different modalities (NIR vs. VIS). The authors reported that there was a significant improvement in the face verification results from the developed approach. Chen et al. [27] developed a method of synthesizing the VIS images from NIR images based on learning the mapping between images captured at different spectral bands.

- Projection Learning Based Infrared Face Matching: Klare et al. [28] performed cross-spectral matching between NIR and VIS images for the database collected at short distance of $0.7 \mathrm{~m}$ under controlled environment conditions. Maeng et al. [29], performed cross distance and cross-spectral matching between VIS and NIR band. The Long-Distance Heterogeneous Face (LDHF) database is presented, which contains face 
images collected in an outdoor environment at distances of $60 \mathrm{~m}, 100 \mathrm{~m}$, and $150 \mathrm{~m}$, with both visible light (VIS) face images captured in daytime and NIR face images captured in nighttime. Yi et al. [30], developed a learning based approach where canonical correlation analysis (CCA) based method was used in order to learn the correlation between NIR and VIS images. In their study, the authors used the short distance (1.5m) indoor database as the gallery set. In [31], cross distance and cross-spectral matching for short and long range distances was performed, in both daytime and night time environments (VIS $1 \mathrm{~m}$ and NIR at $60 \mathrm{~m}, 100 \mathrm{~m}$ and $150 \mathrm{~m}$ ). In this the authors used the SIFT descriptors for the feature extraction and LDA based subspace to minimize the intra-subject differences due to the modality difference. They used the CASIA HFB (NIR-VIS) database at a distance of $1.2 \mathrm{~m}$ to train the system and long distance heterogeneous face (LDHF)-DB long range distance data collected for testing. In [7], a night time imagery system was designed with the capability to acquire images at long-range stand-off distances in NIR band. All NIR face images were acquired at night time and at four different standoff distances of $30 \mathrm{~m}$, $60 \mathrm{~m}, 90 \mathrm{~m}$ and $120 \mathrm{~m}$. Using the face datasets generated for each standoff distance, intra distance and spectral face matching experiments were performed.

- Fusion Based Infrared Face Matching: Kang et al. [39] developed an image restoration (dictionaries) method for cross-spectral matching. To generate the dictionary developed algorithm is trained using low-quality face images (150m NIR) and their corresponding high-quality face images ( $1 \mathrm{~m}$ NIR). Their developed method is based on using good quality face images to train the system but in real time applications it is not always the case that the FR system operators are provided with good and low quality face image corresponding to the same individual to train the system. Omri et al. [37] developed a method to fuse the images collected under different spectral bands (VIS and NIR) at the score level. To fuse the scores they used weighted sum, PCA, empirical mode decomposition and wavelet transform methods. The authors also established a comparison to evaluate each fusion method used. They further extended their work to a long range database collected under challenging conditions at $60 \mathrm{~m}, 100 \mathrm{~m}$ and $150 \mathrm{~m}$ distances [38]. They used short distance (1m) VIS and NIR good quality face images as the gallery set and for 
Table 2.1: Previous work related to heterogeneous face matching.

\begin{tabular}{|c|c|c|}
\hline Related Papers & Distance Range & Cross Spectral Matching Approach \\
\hline Liu et al. [26] & $0.5 \mathrm{~m} \sim 1.2 \mathrm{~m}$ & Synthesis Based \\
Wang et al. [32] & $\sim 1.5 \mathrm{~m}$ & CCA \\
Zhang et al. [33] & $\sim 1.5 \mathrm{~m}$ & Face Analogy \\
Chen et al. [27] & $\sim 1.5 \mathrm{~m}$ & Sparse Representation \\
& & Learning Mapping \\
Yi et al. [30] & $\sim 1.5 \mathrm{~m}$ & Subspace Based \\
Klare et al. [28] & $\sim 0.7 \mathrm{~m}$ & PCA/LDA/CCA \\
Maeng et al. [29] & $1 \mathrm{~m}, 60 \mathrm{~m}$ & HOG-LBP \\
Zhu et al. [34] & $\sim 1.5 \mathrm{~m}$ & DoG-SIFT \\
Goswami et al. [35] & $\sim 1.5 \mathrm{~m}$ & Log-DoG \\
& & LBPH and LDA \\
Raghavendra et al. [36] & $\sim 1.5 \mathrm{~m}$ & Fusion Based \\
Omri et al. [37] & $\sim 1.5 \mathrm{~m}$ & Particle Swarm Optimization \\
Omri et al. [38] & $1 \mathrm{~m}, 60 \mathrm{~m}$, & DWT multispectral \\
& $100 \mathrm{~m}, 150 \mathrm{~m}$ & DWT multispectral \\
Kang et al. [39] & $1 \mathrm{~m}, 60 \mathrm{~m}$, & \\
& $100 \mathrm{~m}, 150 \mathrm{~m}$ & Dictionary: LLE \\
Maeng et al. [31] & $1 \mathrm{~m}, 60 \mathrm{~m}$, & Subspace Based \\
Bourlai et al. [7] & $100 \mathrm{~m}, 150 \mathrm{~m}$ & SIFT-LBP \\
& $1.5 \mathrm{~m}, 30 \mathrm{~m}, 60 \mathrm{~m}$, & \\
Bourlai et al. [40] & $1.5 \mathrm{~m}, 30 \mathrm{~m}, 120 \mathrm{~m}, 60 \mathrm{~m}$, & CSU \\
& $90 \mathrm{~m}, 120 \mathrm{~m}$ & CSU \\
& &
\end{tabular}

the developed fusion method they selected the long range face distance data (probe sets) collected in both bands.

\subsubsection{Developed Solution for Heterogeneous Face Matching System}

Most of the work reported in the literature in the area of heterogeneous face matching in the VIS and NIR bands is based on learning subspace methods. To generate the subspace [6, 7, 26, 28, 29, 39], good quality face images, collected at a short distances (i.e. $1.0 \mathrm{~m}$ in both VIS and NIR bands) were used for training and long distance data (60m, 100m and 150m) was used for testing. However, in the case of operational FR scenarios, we are not always provided with good quality probe and query face images. This work is an effort to solve a more complicated problem as dealing with a database captured at night time environments and variable long standoff distances, starting from $30 \mathrm{~m}$ to $120 \mathrm{~m}$, in $30 \mathrm{~m}$ intervals. 
- One kind of feature set is not adequate. Fusion of complementary feature sets can provide better performance results for FR system (for example fusion of local and global feature sets). The key challenge is the selection of most efficient and discriminative feature descriptors and fusion scheme. In this work, a Multi-Feature Scenario Dependent Fusion scheme is developed.

- An image restoration method to solve the problem of dealing with low quality face images captured at long ranges is developed. The method is based on image de-noising (DN) and super-resolution (SR) techniques, it will be discussed in the methodology section in Chapter 4.

\subsection{Demographic Filtering}

To deal with the problem of poor performance of face recognition system, one of the solution is to use data filtering in terms of demographic information. Filtering is based on the information about the user like weight, height, age, gender, ethnicity and eye color [41]. These traits are also referred as soft biometric traits and provide several advantages such as:

- Complement the identity information provided by the primary biometric identifiers [42].

- Soft biometric information can be obtained for the images acquired at low resolution, at a distance without subject's cooperation and making them ideal for the surveillance applications [43].

- Can improve the speed or search efficiency of the biometric system.

- Can be used for tuning the parameters of the biometric system.

\subsubsection{Reported Work}

These traits have been regularly used by the biometrics research community in various applications [44-46]. Jain et al. [42], utilized the soft traits like ethnicity, weight, gender, height and based on the results they concluded that usage of soft biometrics can significantly 
improve the recognition performance of fingerprint biometric system. The soft biometric traits can also be used for tuning the parameters [47]. The authors proposed to tune the threshold on the matching scores based on the grouping of the data into Male or Female, Asian or Caucasian, etc. groups.

- Demographic Information Based Face Matching: Soft biometric traits are physical, behavioral and human characteristics such as gender, ethnicity, height, weight, and skin color [41]. Some soft biometric traits such as height, weight, and age, change over time [48]. However, traits such as gender and ethnicity are permanent. Mery et al. [45] reported that the usage of soft biometric traits can increase the performance of biometric systems. The authors developed a new approach called adaptive sparse representation of random patches. The developed patch based approach is used to recognize facial attributes such as gender, race, beard, and disguise. The authors made a comparison with other available methods (SVM-RBF, Adaboost) and reported that their system outperformed Adaboost and SVM-RBF based methods. They used AR, UND and FRGC 2.0 databases, which consisted of visible and thermal bands. Park et al. [46] used soft biometric information within an existing biometric system to improve the overall performance of face recognition. The authors demonstrated that soft traits can provide more valuable information in cases where a face image is occluded or captured at challenging viewing angles.

- Automatic Stratification of Demographic Information: Predicting these soft biometric traits can be done reasonably well by human observers. However, when dealing with large datasets this process needs to be performed fast, automatically, and efficiently. The capabilities of existing biometric systems, when performing the gender and ethnicity stratification in controlled conditions, e.g. indoors, outdoors, during day time, at short ranges etc., can result in operationally acceptable stratification rates.

However, automated stratification or demographic filtering, when working at night time environments, under un-controlled conditions and when the subject's face is captured at long standoff distances, is a very challenging task, especially when the face images are captured using different spectral band imaging sensors. 
There are heterogeneous FR systems designed to automatically classify the gender and ethnicity classes [49] for a database collected under controlled environments, and at short standoff distances. The authors developed the gender and ethnicity stratification algorithm based on encoding gradient information on Gabor-transformed images. To perform the estimation of the gender class, authors used AR database, which was composed of visible band face images, including frontal view face with variable facial expressions and different illumination conditions. To perform the estimation of ethnicity class, they selected the Morph and CAS-PEAL databases. The Morph database contains metadata in the form of the gender, ethnicity, age, weight, and height.

Chen et al. [50], developed an automated method for the gender stratification. They selected a face database collected in controlled conditions in the thermal band and CBSR database collected in NIR band in a short range distance $(0.5 m-1.2 m)$. To perform the stratification, features were extracted based on LBP, PCA methods and further these features were used for the stratification using an SVM, an LDA-based and a random forest classifier. Lagree et al. [51], developed a method to predict the gender and ethnicity class from the features of iris texture. Based on the results, the authors reported that the prediction of gender is more difficult than the prediction of ethnicity.

Levi et al. [52] developed a deep learning based method for the classification of gender and age for the wild database collected in the visible band. The authors reported the classification results for the age and gender using the Adience benchmark, which consists of images collected from smart-phone devices. Based on the experimental results, they concluded that convolutional neural network can be used for the age and gender classification.

\subsubsection{Developed Solution for Demographic Filtering}

A deep learning based, scenario-dependent, and band-adaptable (it is working well for both visible and NIR face images) algorithmic approach for the demographic filtering of soft biometric traits in terms of gender and ethnicity is developed. The impact of soft biometric traits in terms of gender and ethnicity is explored for the purpose of improving cross-spectral face recognition (FR) performance. 


\subsection{Image Filtering}

Face database from a surveillance cameras are captured during day or night time, indoors or outdoors, behind tinted glass and at different distances etc. An automatic image filtering, can help to identify the suspect more efficiently and accurately where each probe image is only compared against the gallery images with similar group. For example, probe images labeled with indoor class are compared against only the gallery images with similar group. The manual filtering can be done by humans, such a process is time consuming, especially when dealing with large datasets. Therefore, an automatic way of classifying images into a specific scenario is needed.

\subsubsection{Reported Work}

Vailaya et al. [53], developed an image classification approach in visible band, where low level features are extracted before a binary classifier is used. The authors reported the results for hierarchical classification, where first images are classified into indoor or outdoor, outdoor images are further classified as city or landscape, and, then landscape images are classified into a forest, sunset or a mountain category. Recently, deep convolutional neural networks have achieved great success in the area of computer vision, machine vision, image processing and biometrics for the classification of scenes, object recognition, detection, face authentication and quality assessment. Gupta et al. [54] developed a probabilistic neural network (PNN) based approach for the classification of indoor vs. outdoor visible band images. The segmentation is performed using C-means clustering and features (i.e. color, shape and texture) are extracted from each image segment. In [55], authors developed deep learning method for the scene labeling. The authors reported the results for indoor and outdoor classification. To perform the classification, authors selected a database of 1000 indoor and outdoor images (landscape and city scenes). In [56], a deep CNN for image classification is developed. The authors selected the ImageNet database of over 15 million images and based on the classification results they reported that their $\mathrm{CNN}$ is capable of classifying highly challenging database. Sarkar et al. [57] developed a deep feature based face detector for mobile devices using front-facing 
cameras. The authors applied their developed method on the database collected under varying illumination conditions, poses and partial faces. They reported that the deep feature method outperformed the traditional methods and the developed system can be implemented for offline systems.

For the classification of multi-wavelength images into individual wavelengths, in [58] authors developed the bag of features method to classify the images. This method generates a codebook or dictionary. They implemented this method using visible images to classify the objects based on a selected set of features. The implementation of this method is a very challenging task where multi-wavelength classification is required. The problem is that this method suffers from high complexity to discriminate among various face features extracted from SWIR band images. The limitations of this method on this challenging face database were complexity in terms of the classes and lack of geometrical representation [59]. Namin et al. [60], developed a method to classify images in visible and NIR band captured with a multispectral camera. The subject's face images look similar when captured using a conventional camera but are more easier to distinguish when captured using a multispectral camera. The classification was performed using local features and an SVM classifier.

\subsubsection{Developed Solution for Image Filtering}

Most of the existing classification systems are based on image scene classification into indoors or outdoors and those result in operationally acceptable classification rates. This work is an effort to solve a more complicated problem as dealing with a multi-scenario face database captured at variable illumination conditions in the SWIR band. To address this problem, developed deep features based networks for three different datasets includes:

- Filtering for Multi-Scenario Database in SWIR Band: For multi-scenario database, the face images are captured under challenging conditions [61]. To facilitate recognition performance, knowing the specific image category (based on its origin) is important in order to set the proper parameters for image quality prediction, and face and eye detection. The question is how we can automatically classify the multi-scenario face data. Hence, a scenario-dependent and sensor adaptable CNN based, multi-scenario data fil- 
tering framework is developed, where hierarchical data filtering is performed as follows: Level 1, face images are classified into Indoor or Outdoor; Level 2, face images are further classified into those captured behind different types of tinted glass or not; and, finally, Level 3, face images are classified into those captured when active illumination was used or not.

- Filtering of Multi-wavelength Database into Individual Wavelength: Methodological approach that manages to efficiently classify multi-wavelength images into the right wavelength (1150 to $1550 \mathrm{~nm})$ is developed.

- Filtering for Multi-Sensor Database: A deep network based framework is developed. Tri-level hierarchical classification is performed as follows: Level 1, face images are classified based on phone type; Level 2, face images are further classified into indoor and outdoor images; and, finally, Level 3, face images are classified into close (1m) and far, low quality, (10m) distance.

\subsection{Statistical Hypothesis Testing for Face Matching}

\subsubsection{Reported Work}

In previous work, researchers selected analysis of variance (ANOVA) method for the selection of features [62]. Guo et. al. [63] selected statistical methods, such as mutual information technique to select the most relevant features for gait recognition. To perform the experiments in [63], the Southampton HiD gait database was selected. In [64], the authors applied the t-test to select the most significant features. These features were extracted using the LBP and HOG [65] feature descriptors. The feature selection was performed based on t-test (to select the most significant features) and, finally, those selected features were used for the gender classification using a support vector machine based classifier. Franceschi et. al. [66] developed a nonparametric statistics approach for the selection of features. Based on the developed approach, the authors rejected the non-significant features. To perform the experiments, they selected the MIT-CBCL database. Kumar et. al. [67] developed a correlation based feature selection 
method and further selected features from hand images. Their method was evaluated using naive Bayes, decision trees, $\mathrm{kNN}$ and SVM classification schemes.

\subsubsection{Developed Solution}

The main challenge to perform statistical analysis in heterogeneous FR scenarios is the selection of the type of statistical tests (parametric or non-parametric). Parametric tests (ANOVA) are based on the assumptions of normality of the distributions and homogeneity of variances. This assumption is not valid for the biometric applications. To deal with this problem, in this work, a series of studies are conducted to select the most suitable type of statistical test for the challenging face database. To the best of our knowledge, this is the first time the impact of statistical hypothesis tests to find the statistical significance of image restoration, fusion of scores and usage of soft biometric traits (data stratification), before conducting face matching experiments, is investigated. 


\section{Chapter 3}

\section{West Virginia University Heterogeneous Database}

In this chapter, designed and developed systems for the collection of database in VIS, NIR and SWIR band under controlled and un-controlled conditions are discussed. Four different databases collected in our lab are discussed.

\subsection{Night Time Imagery Long Distance NIR Database}

Visible and NIR cameras were used to collect the night time database at long standoff distances:

Visible Imagery Camera: Canon EOS 5D Mark II is used to collect standard RGB, ultra-high resolution frontal pose face images in the VIS spectrum. This digital SLR camera has a 21.1mega-pixel full-frame CMOS sensor and a vast ISO range from 100-6400.

Near Infrared Mid-Range Camera: The mid-range camera system used in this work is a NIR camera (provided by Vumii Imaging Inc.) that operates at $850 \mathrm{~nm}$. It is a unique outdoor perimeter and border surveillance camera system, and its bulk version integrates (i) an NIR LED illuminator that is invisible to the human eye, (ii) a camera with high-magnification optics (continuous optical long-focal length zoom) placed on a precision pan-tilt platform [7, 68].

- Live Subject-Capture Setup and Datasets: The night time imagery system consists of, NIR camera, VIS camera and a range finder (to find the precise distance). The developed 

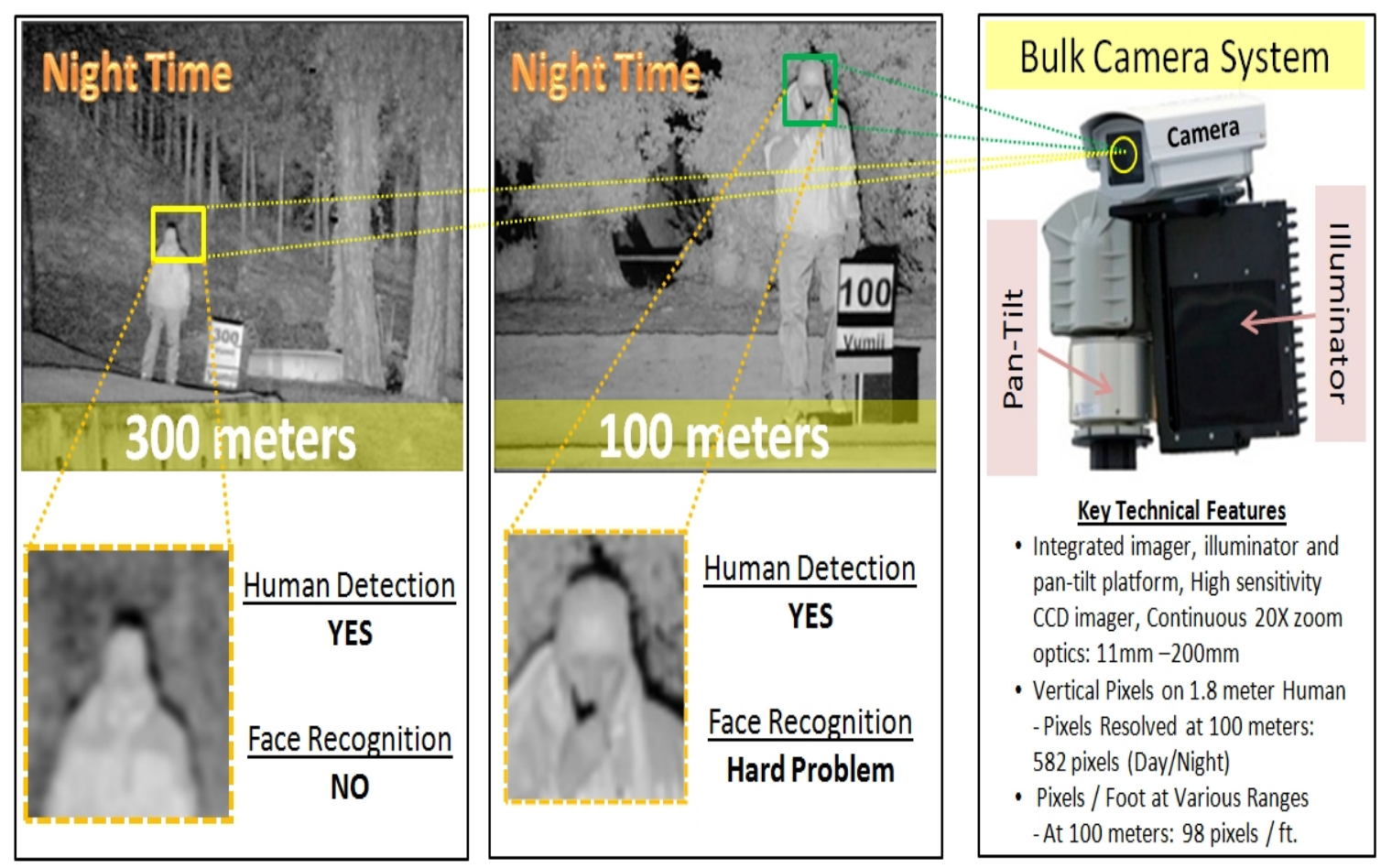

Figure 3.1: Overview of the NIR camera system with the capability to acquire face images day and night at mid-ranges, i.e., from about 20 meters and up to 120 meters. We can see the challenges with nighttime FR at mid-ranges: without active illumination FR is not possible, while with active illumination FR is possible but a very challenging problem, especially at standoff distances further than 60 meters [7].

system was used to collect the face images at four standoff distance of 30, 60, 90 and 120 meters as illustrated in Fig. 3.2. The NIR database was collected in two sessions spanning over a time period of 20 days. The good quality images were taken using the visible camera to generate a baseline database. The database was collected in total from 103 subjects (69 male + 34 female). During the post-processing stage, the videos were first converted into frames. The images where the quality was affected by glare from the car headlights and subjects with closed eyes were discarded from the database.

Regarding other similar databases, there is the one from Maeng et al. [70] collected both indoor VIS and NIR facial images at two different standoffs (1m and 60m). Kang et al. collected the LDHF database (see Table 3.1) that includes both daytime VIS images and nighttime NIR images captured using telephoto lenses and a NIR illuminator at 1m, 60m, 100m, and 150m standoff distances. By contrast our NIR Mid-Range face database is captured using a state-of-the-art NIR imaging system (NIR camera sensor and NIR illuminator) and offers more distances than the LDHF, more data (collected video sequences), and more importantly, demo- 


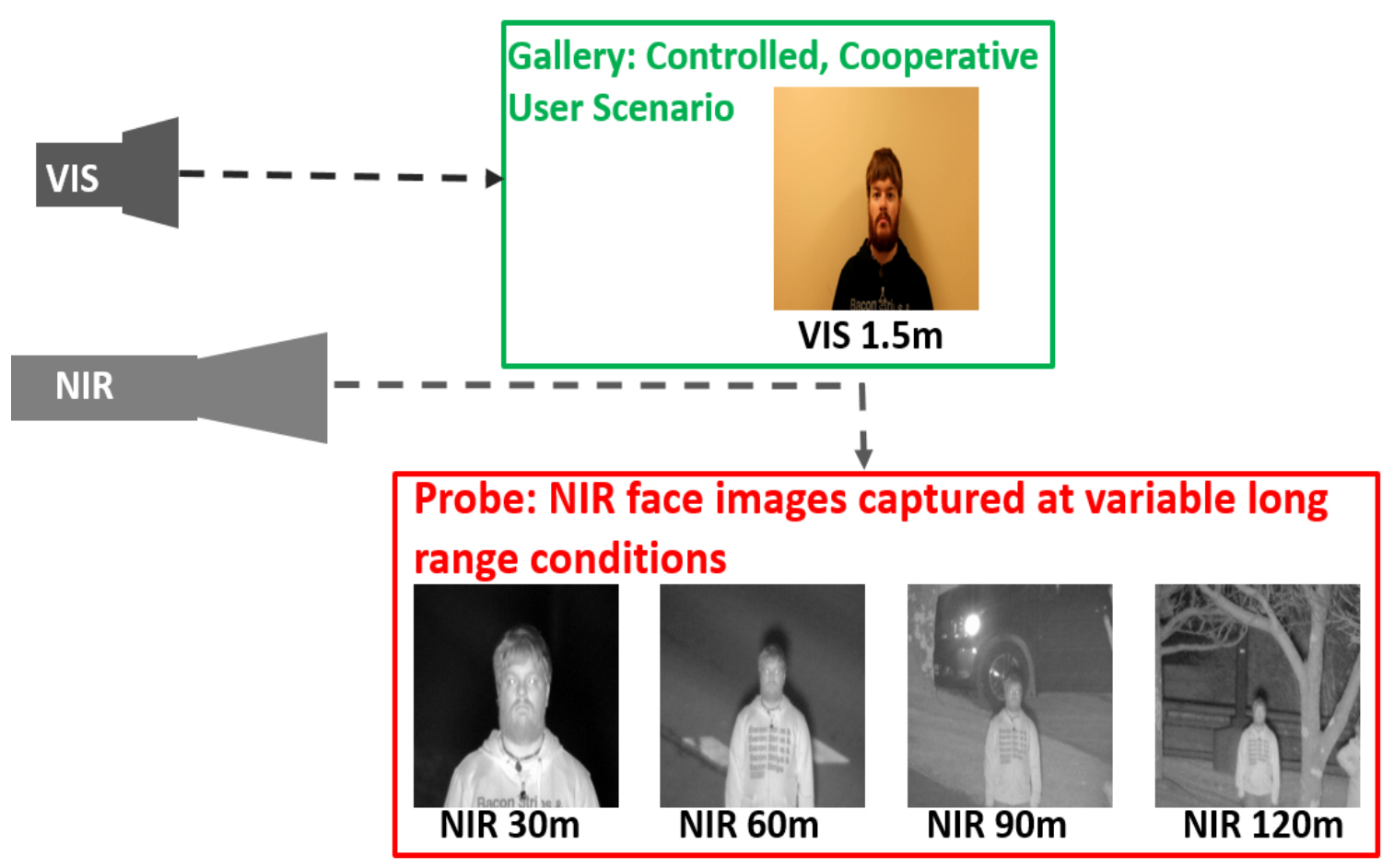

Figure 3.2: The live subject-capture setup using the VIS and mid-range NIR cameras. At the bottom can see a set of face image samples acquired by our system at a night time environment and at variable standoff distances [69].

graphic and environmental information collected per subject. Table 2.1 provides information about relevant work to ours, while Table 3.1 below compares the WVU, NIR Mid-Range and LDHF face databases for cross-distance and cross-spectral face matching.

Table 3.1: Summary of face image databases for cross-spectral and cross-distance matching using long range data. Note: WVU database consists of video sequences for each subject. Still images were selected from those sequences to perform the FR studies. In this work $103 \times 4$ NIR outdoors (2 for gallery and 2 for probes) and 103 visible indoors face images per subject were used.

\begin{tabular}{|c|c|c|c|c|c|c|}
\hline Database & \# Subjects & \# Images & Sensors & Day/Night & Indoors/Outdoors & Distances \\
\hline LDHF[71] & 100 & 200 & Visible & Day & Indoors & $1 \mathrm{~m}$ \\
\cline { 2 - 7 } & 100 & 600 & Telephoto, NIR illuminator & Day and Night & Outdoors & $60 \mathrm{~m}, 100 \mathrm{~m}, 150 \mathrm{~m}$ \\
\hline \multirow{2}{*}{ NIR WVU [7] } & 103 & $\mathrm{n} \times 103$ & Visible & Night & Indoors (mugshots) & $1.5 \mathrm{~m}$ \\
\cline { 2 - 7 } & 103 & $\mathrm{n} \times 103$ & NIR System & Night & Outdoors & $30 \mathrm{~m}, 60 \mathrm{~m}, 90 \mathrm{~m}, 120 \mathrm{~m}$ \\
\hline
\end{tabular}




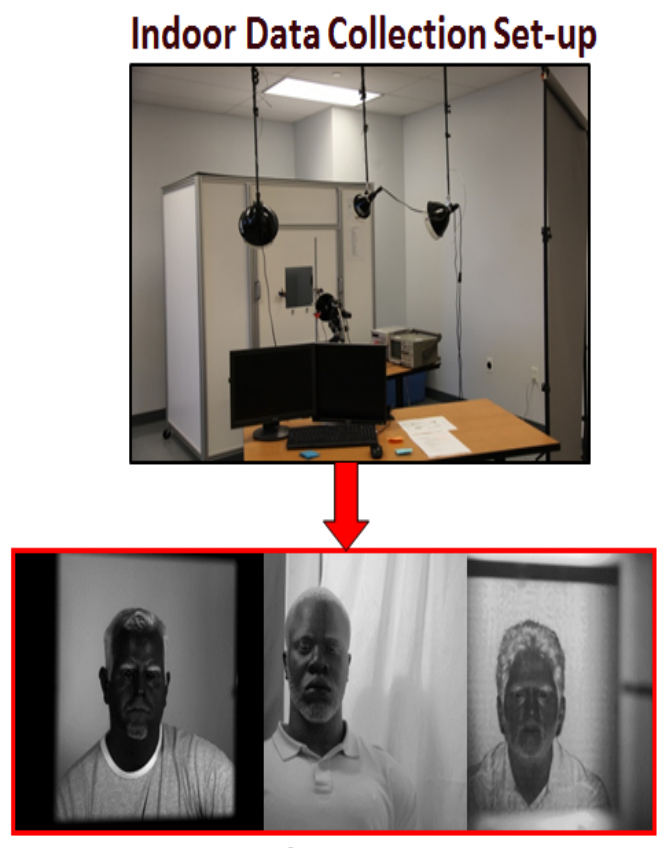

Indoor Images

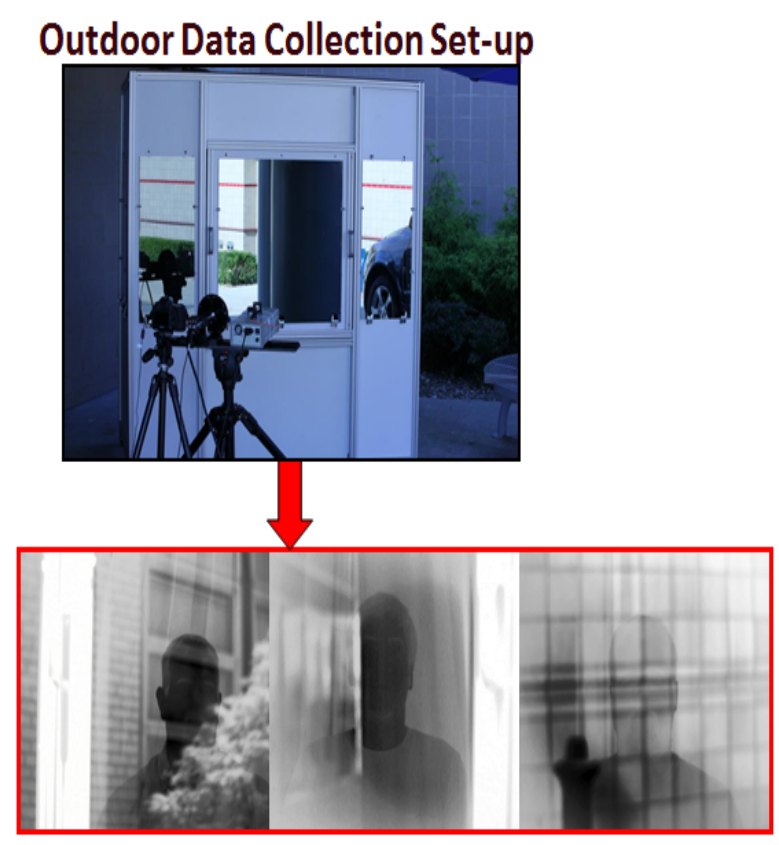

Outdoor Images

Figure 3.3: An overview of the designed data collection set-up to collect the indoor and outdoor face images under challenging conditions [61, 72].

\subsection{Multi-Scenario SWIR Database}

In this work, WVU-MS database [61, 72] is collected in our lab in the SWIR band. The database was captured using Goodrich SU640 (an Indium Gallium Arsenide) video camera featuring high sensitivity in the spectral range from 900-1700 nm and wide dynamic range. A $1550 \mathrm{~nm}$ laser illuminator (eye safe wavelength) was used to capture data behind different types of tinted glass $(0 \%, 80 \%$ and Solar) as shown in Fig. 3.3. The active illuminator acts as a covert device, which is not visible with current night vision technology. However, laser emission is highly visible to SWIR cameras [73].

- Clear with 0\% Film Tint Glass Panel, face images were collected through 0\% tinted glass panel.

- Clear with $80 \%$ Film Tint Glass Panel, face images were collected through $80 \%$ tinted glass panel.

- Solarcool (2) Gray-lite Glass Panel, face images were collected through solarcool graylite glass panel. 
Table 3.2: Database distribution (number of images) for each level.

\begin{tabular}{|c|c|c|c|}
\hline \multicolumn{4}{|c|}{ Level 1} \\
\hline All Data & $\begin{array}{c}\text { Indoor } \\
980\end{array}$ & $\begin{array}{c}\text { Outdoor } \\
758\end{array}$ & $\begin{array}{l}\text { Total } \\
1738\end{array}$ \\
\hline \multicolumn{4}{|c|}{ Level 2} \\
\hline $\begin{array}{l}\text { Indoor } \\
\text { Outdoor }\end{array}$ & $\begin{array}{c}\text { No Glass } \\
140 \\
379\end{array}$ & $\begin{array}{c}\text { With Glass } \\
840 \\
379\end{array}$ & $\begin{array}{l}\text { Total } \\
980 \\
758\end{array}$ \\
\hline \multicolumn{4}{|c|}{ Level 3} \\
\hline $\begin{array}{l}\text { Indoor } \\
\text { Outdoor }\end{array}$ & $\begin{array}{c}\text { Without } \\
\text { Illuminator } \\
420 \\
189\end{array}$ & $\begin{array}{c}\text { With } \\
\text { Illuminator } \\
420 \\
190\end{array}$ & $\begin{array}{l}\text { Total } \\
840 \\
379\end{array}$ \\
\hline
\end{tabular}

The database was collected in a photograph booth as shown in Fig. 3.3 for three different scenarios: (i) Indoor and Outdoor environments under varying daytime and nighttime lighting conditions, (ii) With Glass panels (0\%, 80\% tinted and solar glass) and No Glass and, (iii) With or Without $1550 \mathrm{~nm}$ SWIR Active Illuminator (see Fig. 3.3). In total, the database consists of face images of 140 subjects. The distribution of the database is summarized in Table 3.2 .

\subsection{Single Sensor Multi-Wavelength SWIR Database}

To collect the multi-wavelength face images, a SSMW system was designed and developed [3, 74]. The main components were; a SWIR Goodrich camera, a 5-position rotating filter wheel, a servo-motor and a reflective sensor. Goodrich camera SU640 is an Indium Gallium Arsenide (InGaAs) video camera featuring high sensitivity and wide dynamic range. The spectral range is from $700-1700 \mathrm{~nm}$ wavelength. A 5-position filter wheel was used (manufactured by Optec, Inc.). The filters were selected from $1100 \mathrm{~nm}$, and goes up to $1650 \mathrm{~nm}$, using filters that each have $100 \mathrm{~nm}$ band pass (FWHM) and centered at 1150,1250, . , $1550 \mathrm{~nm}$. A DC motor was used to rotate the filter wheel and optical reflective sensor was used to trigger the camera.

An empirical optimization of the set up was performed including the selection of the light source, synchronization of camera with wheel etc., to acquire the good quality images [74]. The 


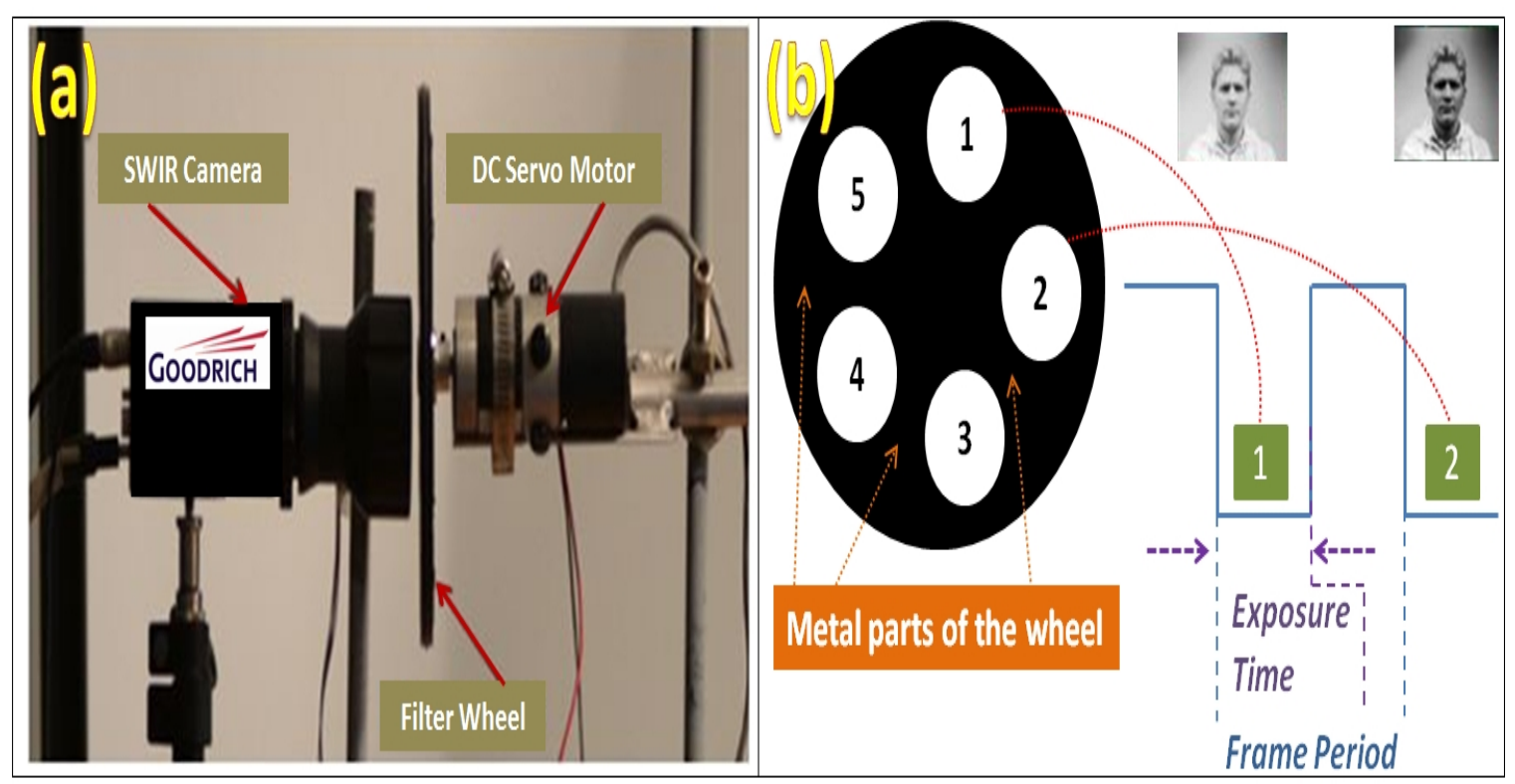

Figure 3.4: (a) Side view of camera setup with the 5-position filter wheel positioned in front of the camera. (b) Example diagram illustrating the synchronization of the camera with the filter wheel.

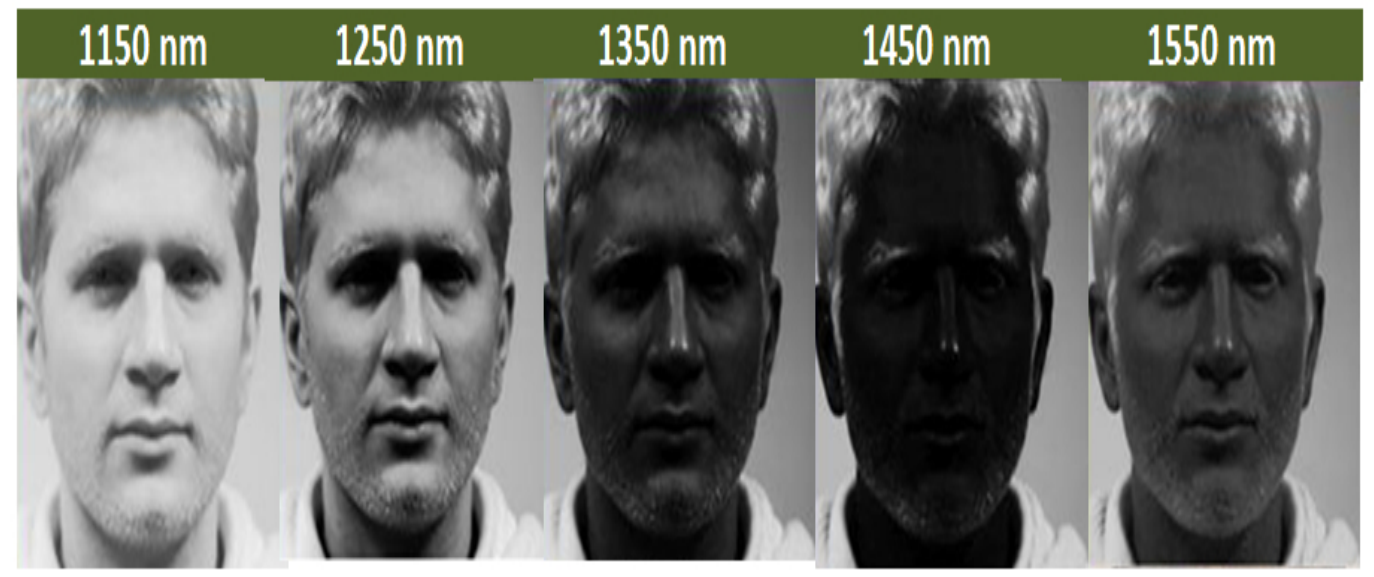

Figure 3.5: Face images captured using designed SSMW system operating in the SWIR band.

face images were captured by the camera, when any of the filters was in front of the camera, avoiding the acquisition of bad images, i.e. at the time instance when the metal parts of the wheel (that is between two successive circular filters on the wheel) were between the camera and the target (see Fig. 3.4(b)).

The constructed wheel, placed in front of the SWIR camera, has five filters, i.e. 1150, 1250, 1350, 1450 and $1550 \mathrm{~nm}$ (see Fig. 3.5). During data collection, focused the camera at $1350 \mathrm{~nm}$ and collected the face images of 30 subjects [3]. The data collection was performed in 2 sessions on different days. Each session took 25 minutes, while both sessions required 
50 minutes in total. In each session collected 275 frames per subject (i.e. 5 set of selected wavelengths $\times 55$ images per wavelength) [74]. The face images collected are left profile, right profile, and full frontal.

\subsection{Multi-Sensor VIS Database}

A multi-sensor visible database (DB1) was collected to perform the face matching experiments using four phones. Face videos were collected indoors, outdoors, at a standoff distance of $1 \mathrm{~m}$ and $10 \mathrm{~m}$ as shown in Fig. 3.6. In total, the database consists of 100 subjects. For each
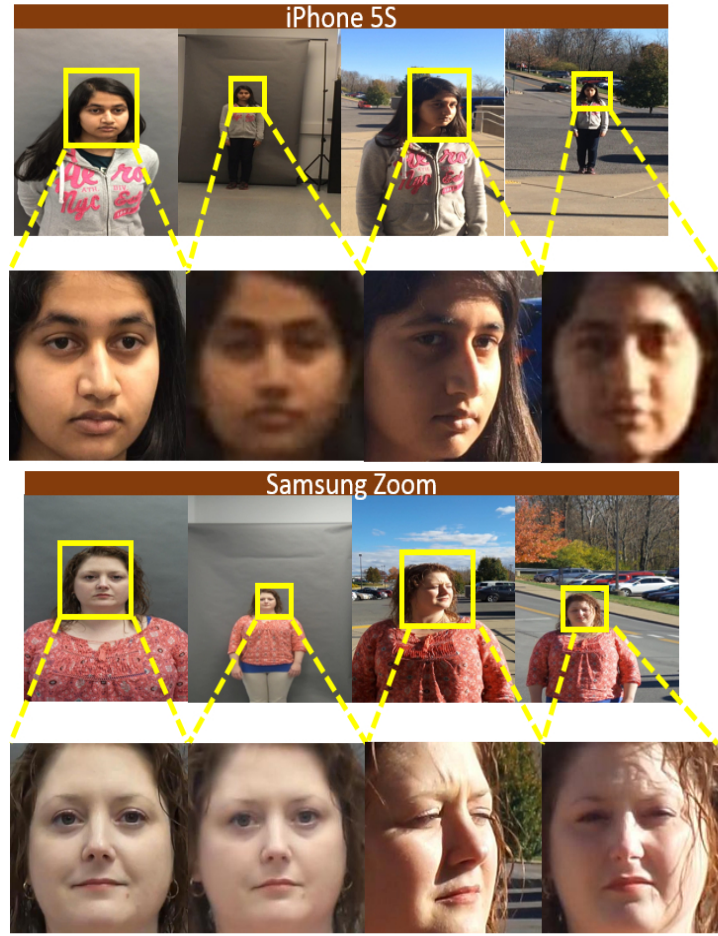
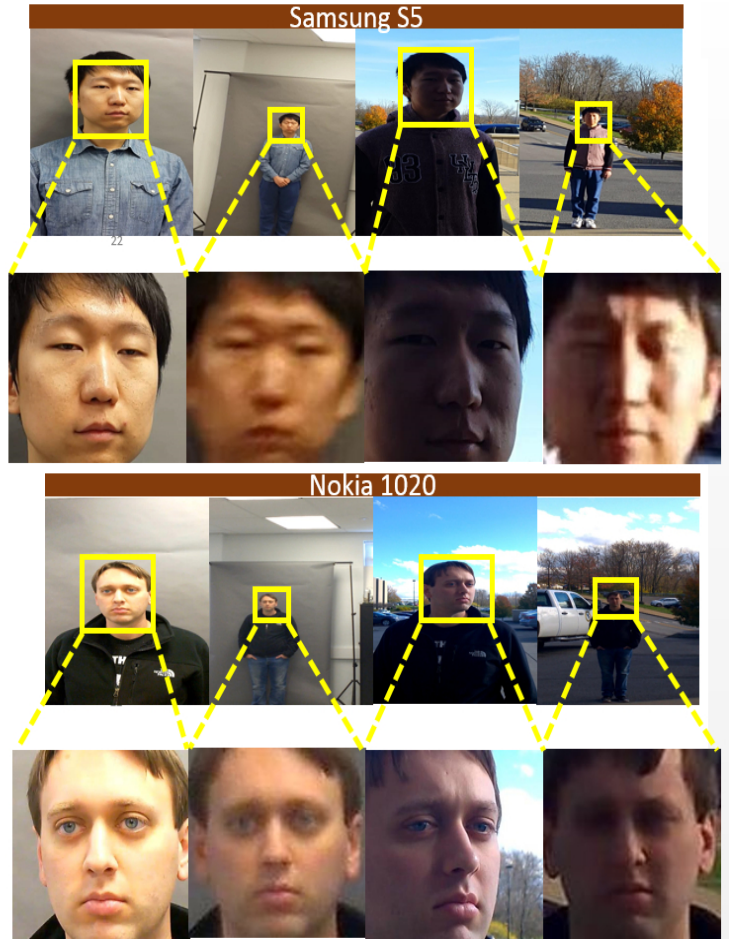

Figure 3.6: Multi-sensor database collected under the challenging conditions.

subject, in total 16 videos are collected, including 4 videos ( 2 videos: indoors $1 \mathrm{~m}$ and $10 \mathrm{~m}$ and 2 videos: outdoors $1 \mathrm{~m}$ and $10 \mathrm{~m}$ ) from each phone. Each video consists of around 700 frames and in total almost 11,200 frames from one subject. Each video was captured with head pose position from $-90^{\circ}$ to $+90^{\circ}$. Two scenarios are selected to collect the database.

- Close Distance $(\sim 1 m)$ : Involves both the face and shoulder part of the body.

- Far Distance $(\sim 10 m)$ : Involves the full body images.

Please check in Fig. 3.6 for the database collected for these scenarios. In the left side 
of the figure, the top 2 rows represent, video frames collected from iPhone $5 \mathrm{~S}$ and the bottom 2 rows represent, the video frames collected from Samsung zoom (equipped with $10 \times$ optical zoom to capture close-ups from far distance). In right side of the figure, the top two rows represent the video frames captured from Samsung S5 and bottom two rows represent from Nokia 1020. A multi-sensor database (DB2) collected from 92 subjects in our lab under un-constrained conditions including, outdoors, at standoff distances of 2.5 to 10 meters for Nokia, Samung S5 and iPhone 5S and 25 to 80 meters for Samsung Zoom was used to train the CNN network. 


\section{Chapter 4}

\section{Methodological Approach}

This chapter discusses the algorithms developed for a score level fusion scheme, automated filtering of data information, and restoration of low quality face images. The statistical analysis tests are performed to find the statistical significance of developed image restoration and fusion schemes in cross-spectral recognition.

\subsection{Study1: Developed Multi-Feature Scenario Dependent Fusion Method}

To perform the cross-scenarios face matching, multi-feature scenario dependent fusion scheme is developed. The developed MFSDF system involves four main steps; (i) Pre-processing of images with mask and without mask, (ii) Feature extraction using global and local descriptors such as Gabor, HOG, and LBP, (iii) Learning Subspace, and (iv) Fusion of scores based on decision level scheme.

\subsubsection{Pre-processing}

Geometric normalization of images is performed to compensate for slight perturbations in the frontal pose. In this work, two different methods, with mask and without mask are adopted. To perform the normalization with mask, the technique developed from Academic Face Identification Evaluation System is used. The more detailed information is provided in 
the pre-processing section of baseline face recognition matchers in chapter 5. With mask faces are canonicalized to the same dimension of $150 \times 130$ pixels [75].

For without mask normalization is composed of two main steps; eye detection and affine transformation. The eye center positions were first located and were used to geometrically normalize the images. Based on the located eye coordinates, the canonical faces were automatically constructed by applying an affine transformation. Faces were first aligned by placing the coordinates of the eyes in the same row such that the slope between the right and left eye is zero degrees. Finally, all the faces were canonicalized to the same dimension of $128 \times 128$ pixels.

\subsubsection{Feature Extraction}

Facial representation is defined as the description of facial information. To transform the matrix array for face into column vector, global and local descriptors are extracted.

Local Binary Patterns (LBP): LBP is a gray scale-invariant local image descriptor and used to get the appearance and texture information. It is highly discriminative, efficient and performs well for the FR systems. LBP method performs well even when the background is not uniform and edges are noisy [76, 77].

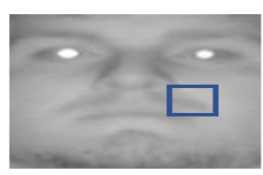

\begin{tabular}{|l|l|l|l|}
\hline 3 & 2 & 1 & 4 \\
\hline 5 & 15 & 29 & 13 \\
\hline 4 & 5 & 25 & 87 \\
\hline 1 & 62 & 40 & 17 \\
\hline
\end{tabular}

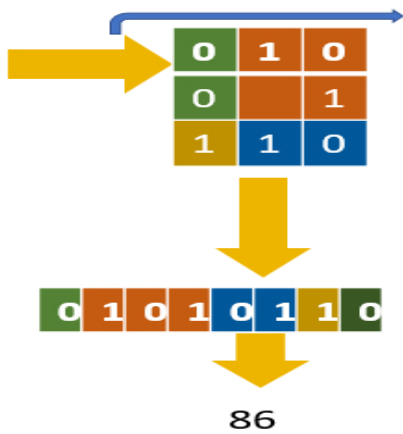

Figure 4.1: LBP Descriptors.

It can filter out noise using the uniform patterns as shown in Fig. 4.1] [40]. The performance of the system decreases when there is a large variation in illumination conditions [77]. To deal with this problem an image restoration method to improve the quality of the images for the database collected under different illumination conditions is developed.

Gabor Method: This method is used to detect high frequency components (edges) in different 


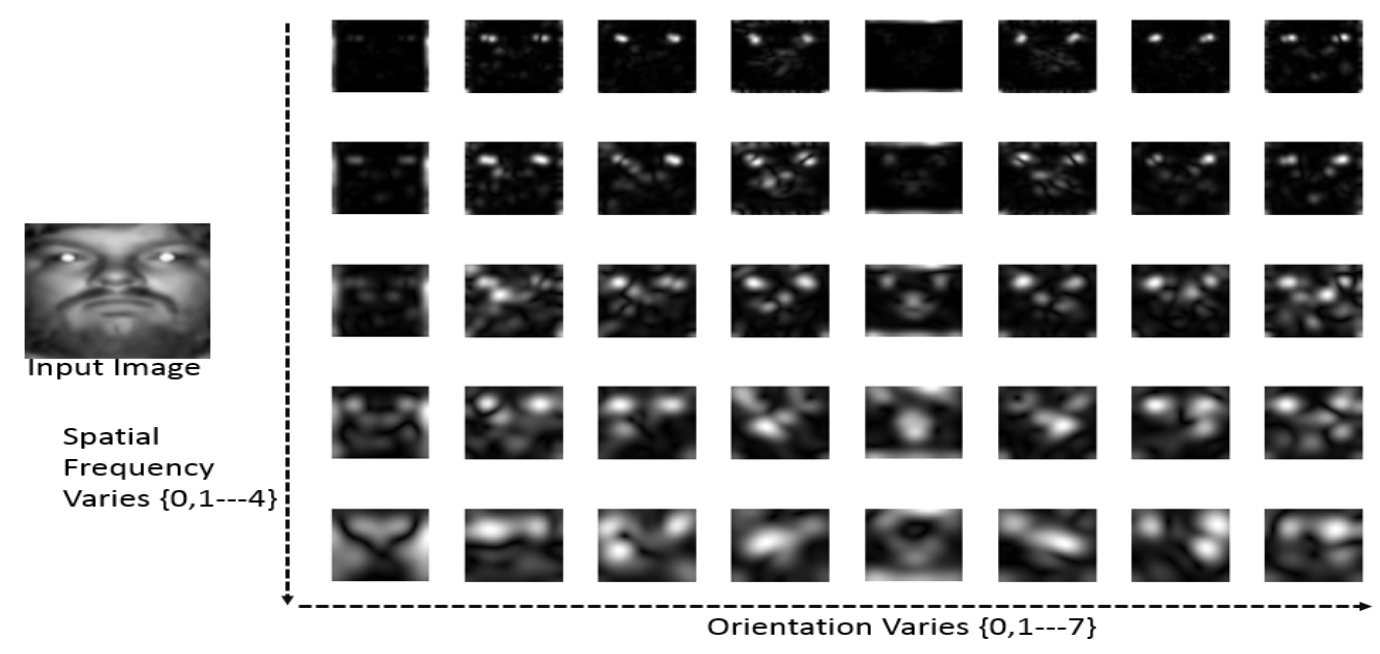

Figure 4.2: Magnitude of Gabor wavelet transformation.

images [78]. This feature descriptor method is most suitable to get the texture information of our NIR face images. Gabor filters, with a selected set of frequency and orientation can be grouped together to form a filter bank. This process is very similar to the way the human visual system works when trying to recognize different objects [77]. The features extracted from Gabor filtered images are more robust to illumination and facial expressions. In this work, 40 Gabor wavelets with the scale value $\nu$ lies in range from $\{0,1,2,3,4\}$ and eight orientations $\mu$ in range of $\{0,1,-, 7\}$ are used (see Fig. 4.2).

HOG Method: $H O G$ has proved to be one of the most successful local shape descriptor and significantly outperforms the available feature sets [65]. The authors used the developed extractor for human detection with images with a large range of poses and backgrounds. The main idea is to estimate the local histograms of image gradient orientation in a dense grid. The appearance and shape of local objects can be well described by the distribution of intensity gradients. Since this method is based on the local cells, which results in significant invariance to the geometric and photometric transformations. In this method, image is divided into cells (overlapping or non-overlapping) and neighboring cells consist of a local region represented as a block. The local shape information (HOG features) is computed for each block. The extracted features of blocks are concatenated into a vector $x$ (components of $x$ are local features of image). The cell size $8 \times 8$ and block size $2 \times 2$ are selected (see Fig. 4.3 ). 


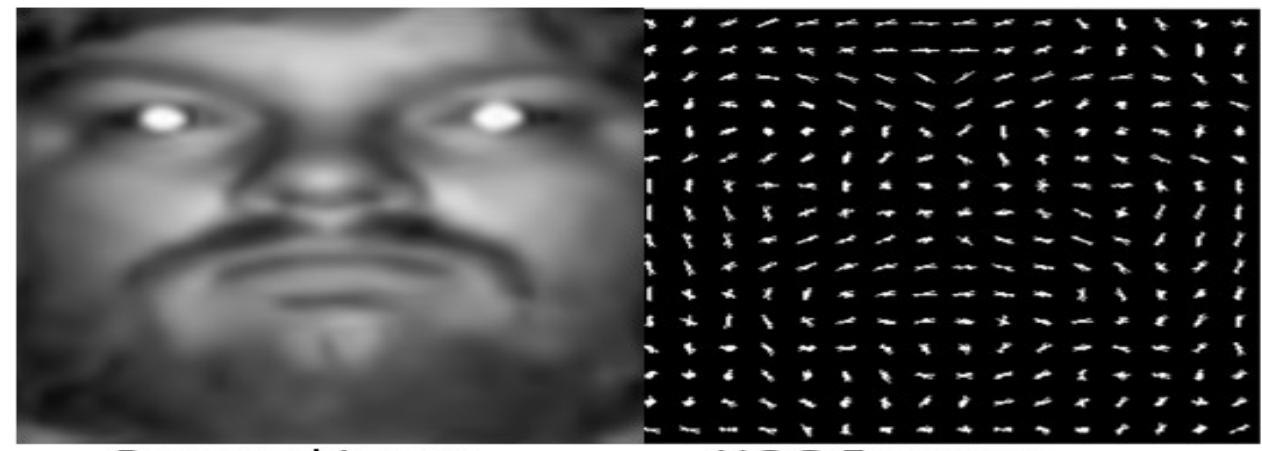

Restored Image

HOG Features

Figure 4.3: Visualization of HOG Features (right) for input restored image (local shape descriptors).

\subsubsection{Feature Subspace}

For NIR and VIS images, the features are extracted based on LBP, Gabor and HOG methods. After the extraction of features in both the cross-spectral bands (NIR and VIS images), the main challenge was to reduce the dimensionality of the feature space. The method used by Tan et al. [77] is adopted. They used LBP and Gabor image feature descriptors. This work is extended to LBP, Gabor and HOG. kernel linear discriminant analysis (KLDA) learning based method is used for the restored images from the developed image restoration method (good quality face images). In KLDA method, the vector $x$ is transformed to $y=w^{T} x$, where $w^{T}$ is the projection matrix. Here, $y$ is linear combination of all the components of vector $x$ and reflects the global features [77].

- Matching For the training phase, both VIS $(1 \mathrm{~m})$ and long range NIR images $(30 \mathrm{~m})$ are selected. Matching was performed only for non-overlapping subjects, those not present in the training set. To perform matching on test set, pre-processing for the restoration of good quality face images, and feature extraction steps are applied.

$$
\begin{gathered}
\Omega_{\text {gallery }}=P^{T} \phi\left(z_{\text {gallery }}\right)=\left(U \Lambda^{-1 / 2} V\right)^{T} k_{\text {gallery }} \\
\Omega_{\text {probe }}=P^{T} \phi\left(z_{\text {probe }}\right)=\left(U \Lambda^{-1 / 2} V\right)^{T} k_{\text {probe }}
\end{gathered}
$$

For a given input face image (gallery/probe), its Gabor, HOG, and LBP features are extracted and separately projected to the optimal discriminant feature space, as given in equation 
(4.1). In the projected space equation (4.1), $\Lambda$ is the diagonal matrix of non-zero eigen values, $U$ is the associated matrix of normalized eigenvectors and $k_{\text {gallery }} \in R^{M}$ is a kernel vector. The variable $V$, for kernel discriminative subspace is computed by solving the LDA eigen decomposition. The features extracted from the visible band face images (gallery set) [77] are projected to $\Omega_{\text {gallery }}$ feature space and probe face image features are projected to an optimal feature space $\Omega_{\text {probe }}$ (separately for each feature). The distance scores are measured using the cosine distance score method (as given in equation (4.3) ) that achieved best results when compared to the L2. The distance scores for each selected feature extraction method are computed.

$$
d_{\text {cos }}\left(\Omega_{\text {probe }}, \Omega_{\text {gallery }}\right)=-\frac{\Omega_{\text {probe }}^{T} \Omega_{\text {gallery }}}{\left\|\Omega_{\text {probe }}\right\|\left\|\Omega_{\text {gallery }}\right\|}
$$

To normalize the scores, a number of well known methods are available, such as z-score, min, max, tanh, etc. [3, 37]. In this work, score normalization is performed using the $z$-score (score values lies between 0 and 1). The normalized scores $z_{L B P}, z_{H O G}$ and $z_{G A B O R}$ are fused based on a decision score level method.

\subsubsection{Fusion of scores}

In most research papers, fusion is performed based on either the sum method or the weighted fusion method [3, 37, 77]. In this work to achieve the best rank identification accuracy, the fusion of scores is performed based on decision level scenarios. Among selected 11 different scenarios: (i) first three are based on single feature descriptor for example only Gabor, only HOG, only LBP, (ii) next six scenarios are based on two features descriptors, using sum and weighted fusion schemes. For sum fusion method, the fusion of normalized distance scores, $z 1$ for Gabor/KLDA, $z 2$ for LBP/KLDA and $z 3$ for HOG/KLDA is performed using four different cases (presented in pseudo code algorithm 1). For sum fusion three combinations are selected: Gabor+LBP, Gabor+HOG, and HOG+LBP. For weighted fusion combinations with two features are selected and weighted are assigned based on the performance of the individual feature descriptor. (iii) Last two scenarios are based on three feature descriptors, using sum and 


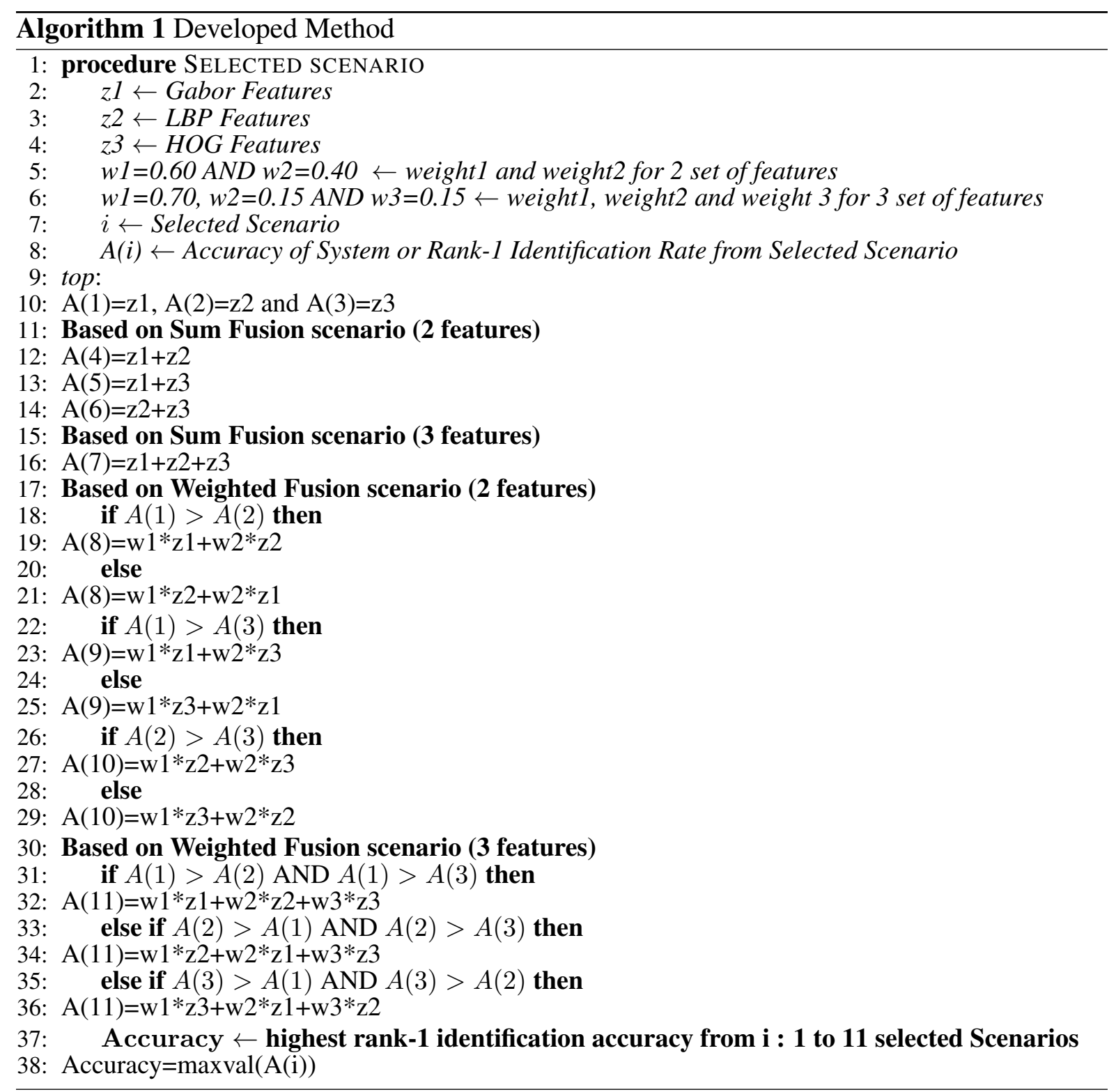

weighted fusion scheme. For sum fusion, Gabor+LBP+HOG is selected and for weighted fusion, weights are assigned to each descriptor based on the performance scores (distance scores) for each individually. Finally, out of these selected scenarios, the one which provided us with best rank-1 identification accuracy or rate is selected. 


\subsection{Study 2: Developed CNN Network for Automatic Predic- tion of Demographic Information}

Deep networks are similar to traditional neural networks, consisting of neurons, learnable weights and biases. The normal network consists of one to two hidden layers and are used for the classification. Whereas, the deep networks have more hidden layers and each hidden layer is connected to many other hidden layers. Deep networks are based on automatic extraction of most suitable higher level features unlike SVM and Bayes, where the selection of features is performed manually.

The deep networks consist of number of convolution layers followed by pooling layers, activation layers and fully connected layers [79].

- Convolution Layer: This is the first layer of the network. This is also defined as a convolutional filter that receives an input image or signal. It is a mathematical operation, where we apply a filter of size $3 \times 3$ or $5 \times 5$ to the patch of the image, as shown in Fig. 4.4. It multiplies each pixel in the filter by the value of the corresponding pixel in the image. In convolution layer each neuron is only connected to few neurons from the previous layers and the same set of weights is used for each neuron. In comparison to fully connected layer in this layer, the less number of connections and weights are responsible for cheap networks in terms of memory and compute power.

\begin{tabular}{lll|ll}
$\mathbf{1}$ & $\mathbf{1}$ & $\mathbf{1}$ & $\mathbf{0}$ & $\mathbf{0}$ \\
\hline 0 & 1 & 1 & 1 & 0 \\
0 & 0 & 1 & 1 & 1 \\
0 & 0 & 1 & 1 & 0 \\
0 & 1 & 1 & 0 & 0
\end{tabular}

Input Image

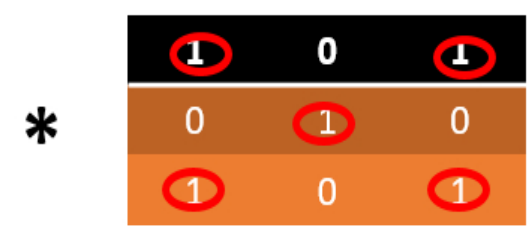

Kernel Filter $(3 \times 3)$

Figure 4.4: Convolution

- Pooling Layer: The max pooling layer, which is applied to perform the down sampling and smoothing to reduce the sensitivity of filters to noise and variation. This layer 
eliminates the non-maximal values and reduces the computation for upper layers. It is invariant to translation and is defined as smart way to deal with dimensionality problem.

- Activation Layer: Activation layer is applied to network to control how the signal flows from one layer to another layer. The most common function of activation layer is Rectified Linear Unit (ReLU). ReLU plays an important role in dealing with the problem of non-linearity. It converts all the negative numbers to zero.

- Fully Connected Layer: In the fully connected layer each neuron is connected to every neuron in the previous layer and each connection has it's own weights [79].

- Softmax Layer: The last layer is Softmax layer that computes scores for each class.

The data filtering is performed for two scenarios, (i) Intra-spectral, where the visible, NIR or SWIR images are selected for both the training and testing, (ii) Cross-spectral, where the visible images are selected for the training and NIR or SWIR images for testing. To perform the intra-spectral and cross-spectral data filtering, the visual geometry group (VGG) CNN architecture [79] is selected. It consists of a number of convolutional layers (a bank of linear filters), followed by max pooling layers and a rectification layer, such as the rectified linear unit (ReLU) along with fully connected layers [52, 79, 80]. The pooling layer computes the maximum of local region (down sampling operation).

\subsubsection{Network Architecture}

In first convolution layer, 20 filters of size $5 \times 5$ are applied to the input, followed by a max pooling layer taking the maximal value of $2 \times 2$ with two-pixel strides. The output of the previous layer is processed by a second convolution layer, with 50 filters of size $5 \times 5$, followed by a max pooling layer with the same stride value. The second layer is further processed by a third convolution layer with 500 filters of size $4 \times 4$. This layer is followed by an activation layer (ReLU) [80]. Finally, a third layer with 500 filters of size $2 \times 2$ is processed by the last convolution layer. The output of this layer is fed to a Softmax layer that assigns a label based on the selected database and training model: 
- For the night time long range NIR imaging system, it will assign a label male or female in terms of gender and Asian or Caucasian in terms of ethnicity class.

- For Multi-Scenario Database in SWIR band, it will assign a label indoors or outdoors, with or without glass and active illuminator on.

- For Single Sensor Multi-wavelength database in SWIR band, it will assign a label 1150 or 1250 or 1350 or 1450 or $1550 \mathrm{~nm}$.

- For Multi-Sensor database in VIS band, it will assign label, phone type, indoors or outdoors and close or far distance.

\section{- Challenges:}

- No pre-trained network model is available to use for this CNN network to perform the classification for the challenging database collected in infrared band (NIR and SWIR). To address this problem, the models are trained on our original database.

- Selection of hyper-parameters (e.g. momentum, epoch, learning rate and step size).

\subsection{Study 3: Developed Image Restoration Scheme}

Face images captured in the NIR band appear different from the face images captured in visible (VIS) band. The long standoff NIR face images were captured outdoors, and in challenging conditions where the background was not necessarily uniform and objects, such as vehicles and trees, may hinder the automatic detection of faces. To remove the difference in appearance between face images captured in NIR and VIS band and to improve the quality of images, an image restoration approach is developed. The pre-processing of images is composed of three main steps: geometrical normalization $(G N)$, image restoration from the developed approach and photometric normalization $(P N)$. In image restoration, the good quality face images are reconstructed from an image enhancement method using a denoising $(D N)$ and a superresolution $(S R)$ method. Finally, $P N$ is used to compensate for illumination variations. The advantage of the developed pre-processing steps is that they can eliminate the irrelevant information while, still preserving face appearance details that are required for face recognition. 
- Geometrical Normalization It is composed of two steps, eye detection and affine transformation and compensates for slight perturbations in the frontal pose as described in Chapter 4 in section 4.1.1. All the faces are canonicalized to the same dimension of $128 \times 128$ pixels.

- Image Restoration Scheme It is composed of a set of algorithms namely $S R$ and $D N$. Both of these algorithms are described below. However, the main questions are, do we need to use them independently or in combination with each other? and which combination will result in better rank-1 identification accuracy in various cross spectral FR experiments?

(1) Super-Resolution: In this work, restoration is performed based on example based $S R$ method [81] and $D N$ method [82]. SR method is based on the single image up-scaling and does not rely on another external example database or source. This method is based on the observation of local self-similarity [LSF] of images, not only globally between different features, but also at localized regions [82].

The same technique developed by Freedman et al. [81] is adopted and the algorithm for our challenging database at long distances is optimized, where an input image $I_{0}$ is interpolated to a high resolution image using custom filters. The up-scaled image $L_{1}\left(L_{1}=\mu\left(I_{0}\right)\right)$ lacks upper frequency band which is proportional to the selected scaling factors (scaling factor value is 1.25 in this work). These missing upper frequency bands are filled using a non-parametric patch based model, which relies on LSF. To generate the exemplar patches, an input image is decomposed into low frequency and high frequency components $L_{0}\left(L_{0}=\mu\left(D\left(I_{0}\right)\right)\right.$. The high frequency prediction is conducted or computed by matching every patch in $L_{1}$ with its most similar patches in $L_{0}$. The low resolution image $\left(L_{0}\right)$ has high frequency content compatible with the interpolated image $L_{1}$. For each patch in the interpolated image, its closest match in the low-passed image is searched, and finally, the corresponding high-pass values to the high resolution image are copied. This task is performed for all the patches in the high resolution image.

(2) De-noising: For the images collected in nighttime environment, at long standoff distances, to improve the quality of images (to remove the noise), a $D N$ algorithm based on wavelets is applied. In equation (4.4), $F(i, j)$ represents the noise free image and $Y(i, j)$ is the image corrupted 


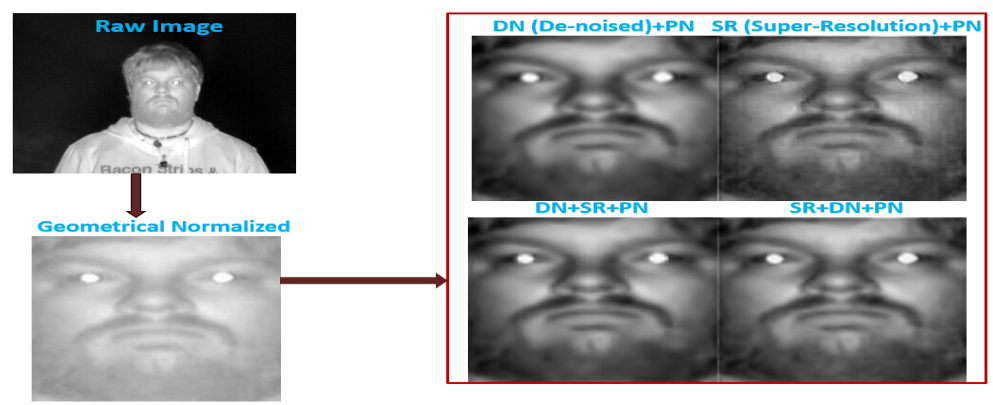

Figure 4.5: Raw image (top left), only geometrically normalized image (bottom left) and restored images from developed algorithm (right).

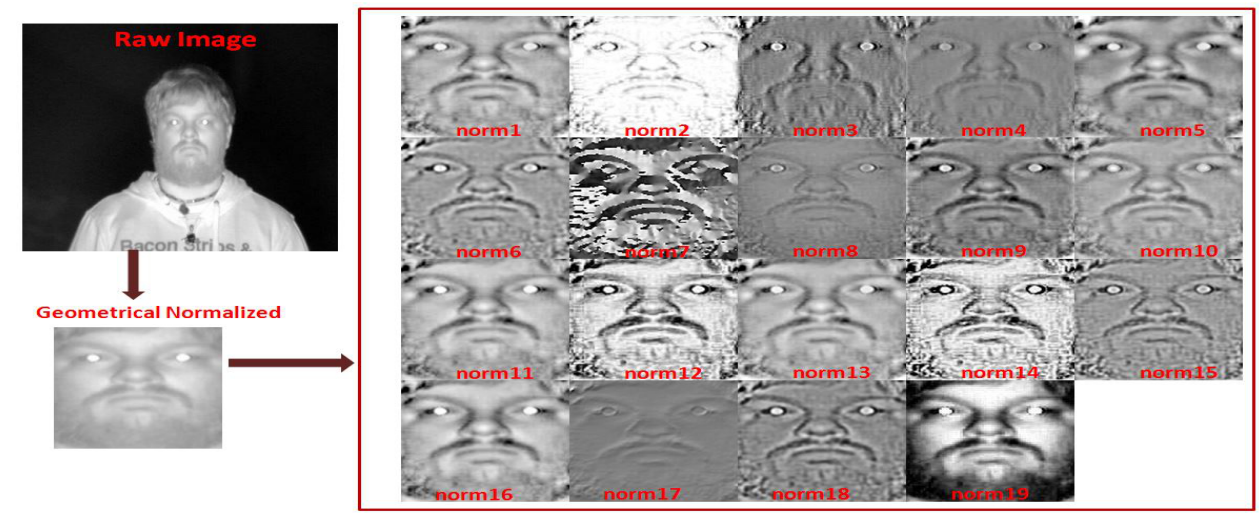

Figure 4.6: Raw image (top left), geometrically normalized image (bottom left) and photo-metrically normalized images from a selected set of normalization techniques [83] (right).

with independent gaussian noise. $Z(i, j)$ is assumed to have a normal distribution with $N(0,1)$.

$$
Y(i, j)=F(i, j)+\sigma * Z(i, j)
$$

The steps involved for this are, (i) Discrete wavelet transform $(D W T)$ is applied to the noisy images by using a Daubechies wavelet, (ii) Hard-thresholding is applied to obtain the estimated wavelet coefficient and, (iii)Inverse discrete wavelet transform (IDWT) is applied to reconstruct the de-noised image from estimated wavelet coefficient.

- Photometric Normalization $P N$ is applied to the raw (only $G N$ ) and restored face images to compensate for changes in illumination conditions. In this work, empirically 19 different $P N$ techniques [83] are investigated including: adaptive non local means (norm 1), adaptive single scale retinex (norm 2) etc. (as shown in Fig. 4.6). To optimize the set up (for each scenario), the $P N$ method that provides us with the best identification rate or accuracy is selected. The 
performance results with rank-1 identification accuracy are shown in the experimental results section (see Figs. 7.1 and 7.2).

\subsubsection{Selection of Pre-processing Combination}

The main challenge is to select the best order of combinations for pre-processing, as we cannot randomly select either $P N, D N$ or $S R$. To deal with this problem five combinations

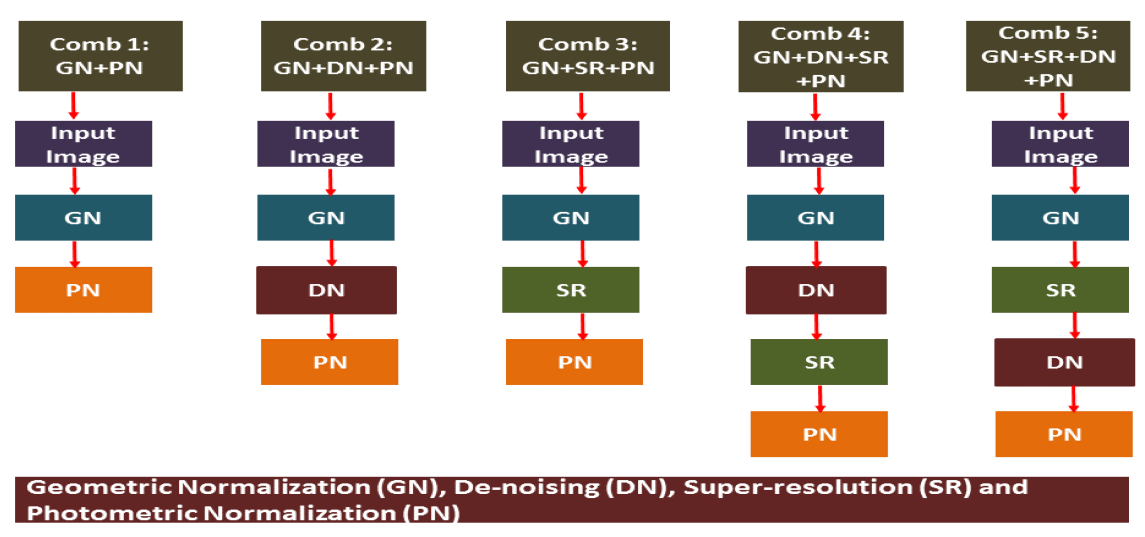

Figure 4.7: Block diagram representation for the selected order of each pre-processing technique.

are selected such as comb1 $(G N+P N), c o m b 2(G N+D N+P N), c o m b 3(G N+S R+P N), c o m b 4$ $(G N+D N+S R+P N)$ and $c o m b 5(G N+S R+D N+P N)$ (see Fig. 4.7). The selection of the order of each of pre-processing techniques is critical and is also of key importance in improving crossspectral matching performances in terms of rank-1 identification accuracy. The results for $L B P$, LTP, Gabor, HOG are shown in experimental results section in Figs. 7.1 and 7.2

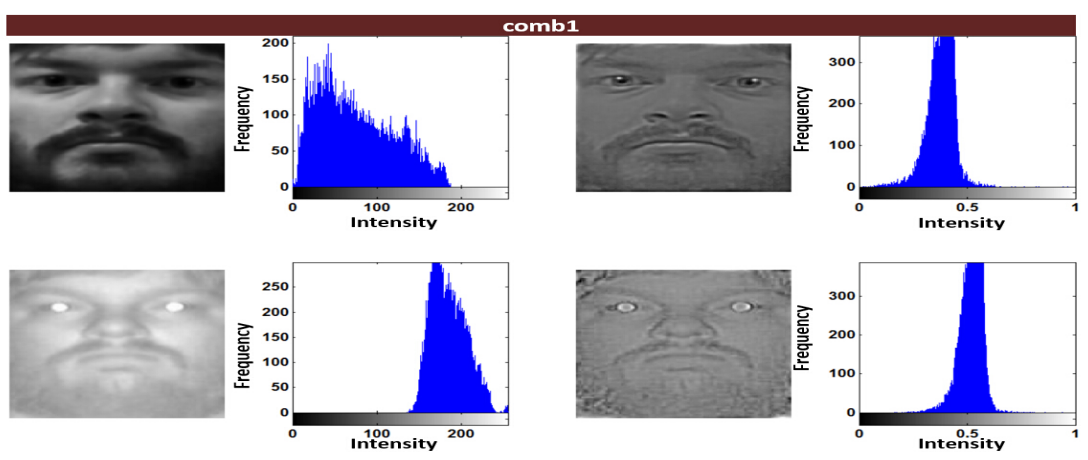

Figure 4.8: For $P N$ method applied to raw images (comb1), gallery (VIS-top right) and probe (NIRbottom right).

First a set of experiments are performed including: applying $P N$ directly on raw images 
combl and then on restored images ( $D N$ and $S R)$. In Fig. 4.8, histogram plots for gallery (VIS) and probe image (NIR), where $P N$ is implemented on raw data and in Fig. 4.9 histogram plots for the $P N$ methods applied to the restored face images are presented. From Fig. 4.8, it can easily be interpreted that there is a large variation between the intensity values for gallery and probe images. Whereas, this variation is minimized when the $P N$ is applied on the restored

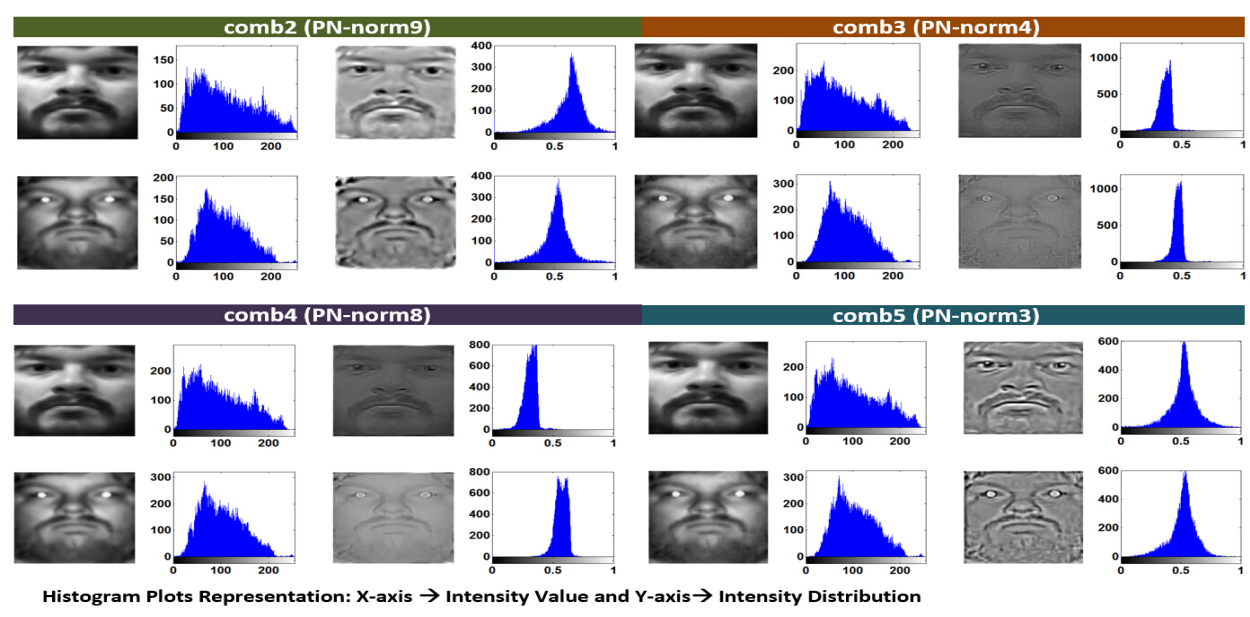

Figure 4.9: For $P N$ technique applied to restored images (using the developed image restoration method).

images (specifically for restored images using comb3 and comb4), as shown in Fig. 4.9. 


\section{Chapter 5}

\section{Multi-Feature Scenario Dependent Fusion}

\section{Scheme for Night Time Long Range}

\section{Imagery System in NIR Band}

In this chapter, first the baseline results from commercial and academic face matchers are presented. Intra-spectral and cross-spectral face matching results for the NIR database collected in night time and at long standoff distances are discussed using the developed multi-feature scenario dependent fusion scheme in study 1 . It is investigated whether the developed approach from study 1 improves the baseline performance.

\subsection{Baseline Face Recognition Tools}

To perform the baseline identification for intra-spectral and cross-spectral scenarios, both commercial and academic softwares are used. For the commercial off-the-shelf (COTS) software, Identity Tools G8 (www.11id.com) provided by L1 Systems is used. For the academic Academic Face Identification Evaluation System (AFIES) provided by Colorado state university [75] is used. The software consists of Principle Components Analysis (PCA) [8486], a combined Principle Components Analysis and Linear Discriminant Analysis algorithm (PCA+LDA) [87], and the Bayesian Intra-personal/Extra-personal Classifier (BIC), using either the Maximum likelihood (ML) or the Maximum a posteriori (MAP) hypothesis [88]. 
While the pre-processing routines employed by the commercial software are not known. For academic software a normalization scheme that compensates for slight perturbations in the frontal pose is employed. It consists of eye detection and sequence of normalization steps including, (i) integer to float conversion, (ii) geometric normalization (lines up human chosen eye coordinates), (iii) masking (crops the image using an elliptical mask and image borders such that only the face from forehead to chin and cheek to cheek is visible), and, (iv) pixel normalization (scales the pixel values to have a mean of zero and a standard deviation of one). Eventually it provides a set of canonical faces that are all warped to the same dimension of $150 \times 130$ pixels [75].

\subsection{Face Recognition Studies}

\subsubsection{Matching Across Different Scenarios}

For face matching the datasets briefly discussed in subsection 3.1 are utilized. Datasets of face images from 103 subjects consists of: a visible (baseline) dataset and four night-time datasets (including non-facial context data) at 30m, 60m, 90m and $120 \mathrm{~m}$, with eight NIR face images per subject. After that the following experiments are performed:

Experiment 1: Visible vs. Visible (intra-spectral) face matching - In this only commercial software is used and it provided the rank-1 identification rates.

Experiment 2: NIR vs. NIR (intra-spectral) and intra-distance face matching - In this experiments are performed when both the gallery and probe images were selected from NIR band. The experiments were conducted for $30 \mathrm{~m} v \mathrm{~s} .30 \mathrm{~m}, 60 \mathrm{~m} v \mathrm{~s} .60 \mathrm{~m}, 90 \mathrm{~m} v \mathrm{~s} .90 \mathrm{~m}$, and $120 \mathrm{~m} v \mathrm{~s}$. $120 m$.

Experiment 3: In this, experiments are conducted for cross-spectral and cross-distance scenarios, i.e. the baseline visible images in the gallery set were matched against the NIR images captured at $30 \mathrm{~m}, 60 \mathrm{~m}, 90 \mathrm{~m}$ and $120 \mathrm{~m}$ respectively.

Note that the identification performance of the system is evaluated through the cumulative match characteristic (CMC) curve. The CMC curve measures the $1: m$ identification system performance, and judges the ranking capability of the identification system. Here it is inves- 
Table 5.1: Summary of total number of face recognition experiments are performed for each dataset, with and without demographic filtering. To perform the FR experiments, two FR crossscenarios are selected cross-distance (CD) and cross-spectral (CS).

\begin{tabular}{|c|c|c|c|c|}
\hline Datasets & $\begin{array}{c}\text { Train/Test } \\
\text { Datasets }\end{array}$ & \# Scenarios & $\begin{array}{c}\text { AFIES, COTS and } \\
\text { Developed MFSDF }\end{array}$ & $\begin{array}{c}\text { \# Total } \\
\text { Experiments }\end{array}$ \\
\hline All & 4 & 3 CD + 4 CS & $6+1+2$ & 252 \\
\hline Male & 4 & 3 CD + 4 CS & $6+1+2$ & 252 \\
\hline Female & 4 & 3 CD + 4 CS & $6+1+2$ & 252 \\
\hline
\end{tabular}

tigated whether the change in distance $(30 \mathrm{~m}$ to $60 \mathrm{~m}, 90 \mathrm{~m}$ and $120 \mathrm{~m})$ and sensor (gallery in VIS and Probe in NIR), affects the performance of face recognition system. For the database collected under un-controlled conditions, MFSDF method to improve the identification rank-1 performance of the system is developed.

The experiments aim to illustrate how the baseline NIR FR system operates when compared to a conventional visible FR system (where face images are captured under controlled conditions). We need to know what is the rank-1 identification rate when matching NIR to NIR good quality and long range face images (30m vs. $30 \mathrm{~m})$. Finally, we need to know the extent of which the rank-1 identification rate is affected when standoff distance increases. This is established by performing intra - and cross-distance NIR FR matching when going from $30 \mathrm{~m}$ to $120 \mathrm{~m}$. Cross-spectral and cross-distance matching is also investigated using the same commercial, academic and the developed algorithms.

Table 5.1, describes information the total number of selected datasets, cross-scenarios and face recognition algorithms. For each dataset the $50 \%$ data is randomly selected as training set and rest of data set as testing set. This process is repeated 4 times, using random selection of training and testing set each time. Two cross-scenarios are selected: cross-distance (CD) and cross-spectral (CS). For CD NIR vs. NIR, face matching is performed for 3 sets: $30 \mathrm{~m}$ vs. $60 \mathrm{~m}, 30 \mathrm{~m} v \mathrm{~s} .90 \mathrm{~m}$ and $30 \mathrm{~m} v \mathrm{~s} .120 \mathrm{~m}$. For CS VIS vs. NIR, face matching is performed for 4 sets: $1 \mathrm{~m}$ vs. $30 \mathrm{~m}, 1 \mathrm{~m}$ vs. $60 \mathrm{~m}, 1 \mathrm{~m}$ vs. $90 \mathrm{~m}$ and $1 \mathrm{~m}$ vs. $120 \mathrm{~m}$. Cross-scenarios are investigated using academic, commercial and the developed method. AFIES and developed system are training based methods. Face recognition academic software is based on PCA, PCAEUC, BIC-ML, BIC-MAP, LDA-EUC and LDA-ldaSoft, and the developed method is based on 
Table 5.2: In this table the intra-spectral, intra-distance matching scenarios without demographic filtering for the database collected from 103 subjects are investigated. Experimental results are presented while running all CSU FR algorithms when using 20\%, 40\% and 60\% of the NIR data for training and the rest data $(80 \%, 60 \%$ and $40 \%$ respectively) for testing. When CSU is used, the experiments were run 10 times and the identification rank-1 accuracy presented here are the means. G8 is also tested for each scenario. Note that there is one case $(90 \mathrm{~m})$ where the identification rank-1 accuracy was about $38 \%$ vs. $59 \%$ with the $120 \mathrm{~m}$ data. This was because the experiment was run once and it was noticed that some $90 \mathrm{~m}$ face images selected for matching were more heavily affected by background noise (e.g. passing cars and illumination) due to the time of the collection. There is no overlap of subjects between training and testing.

\begin{tabular}{|c|c|c|c|c|c|c|c|c|c|c|c|c|}
\hline \multirow{3}{*}{ FR Algorithm } & \multicolumn{12}{|c|}{ Intra-Spectral (NIR vs. NIR), Intra-Distance Matching - Rank-1 Identification Accuracy } \\
\hline & \multicolumn{3}{|c|}{$30 \mathrm{~m}$} & \multicolumn{3}{|c|}{$60 \mathrm{~m}$} & \multicolumn{3}{|c|}{$90 \mathrm{~m}$} & \multicolumn{3}{|c|}{$120 \mathrm{~m}$} \\
\hline & $20 \%$ & $40 \%$ & $60 \%$ & $20 \%$ & $40 \%$ & $60 \%$ & $20 \%$ & $40 \%$ & $60 \%$ & $20 \%$ & $40 \%$ & $60 \%$ \\
\hline Bayesian MAP & 0.99 & 0.99 & 0.99 & 0.90 & 0.95 & 0.95 & 0.95 & 0.98 & 0.99 & 0.92 & 0.96 & 0.98 \\
\hline Bayesian ML & 0.99 & 0.99 & 0.99 & 0.99 & 0.99 & 0.99 & 0.94 & 0.96 & 0.98 & 0.91 & 0.95 & 0.99 \\
\hline LDA Euclidean & 0.99 & 0.99 & 1.00 & 0.99 & 0.99 & 1.00 & 0.96 & 0.99 & 1.00 & 0.92 & 0.97 & 1.00 \\
\hline LDA lda_Soft & 0.99 & 0.99 & 1.00 & 0.98 & 0.99 & 1.00 & 0.95 & 0.99 & 1.00 & 0.92 & 0.98 & 1.00 \\
\hline PCA Euclidean & 0.97 & 0.98 & 1.00 & 0.96 & 0.90 & 0.98 & 0.91 & 0.93 & 0.94 & 0.87 & 0.91 & 0.94 \\
\hline G8 & \multicolumn{3}{|c|}{0.95} & \multicolumn{3}{|c|}{0.69} & \multicolumn{3}{|c|}{0.37} & \multicolumn{3}{|c|}{0.5} \\
\hline
\end{tabular}

with and without mask multi feature scenario dependent fusion scheme. Whereas, commercial software is non-training based method. In total, 9 different FR experiments (AFIES -6, COTS -1 and the developed MFSDF -2) are applied. The last column in the table represents the total number of face matching experiments performed for each dataset.

\subsubsection{Baseline and Developed System Matching Results Before Demo-} graphic Filtering

Intra-Spectral and Intra-Distance Face Matching: In this, NIR to NIR, intra-distance face images are compared. The experiments performed are:

- $30 m$ vs. $30 m$

- $60 m$ vs. $60 m$

- $90 m$ vs. $90 m$

- $120 m$ vs. $120 m$

For this purpose G8 (COTS) and the academic software (AFIES) [75]) are employed while the training set size was varied to determine whether larger training sets will result in higher 
Table 5.3: In this table the intra-spectral, cross-distance matching scenarios (without demographic filtering) are investigated. Experimental results are presented when running all FR algorithms using 50\% of the NIR data for training and the rest of the data for testing (database consists of 103 subjects). For each algorithm including, AFIES, COTS and the developed MFSDF, the experiments were run 4 times and the rank-1 identification accuracy is presented here. There is no overlap of subjects between training and testing.

\begin{tabular}{|c|c|c|c|}
\hline $\begin{array}{c}\text { FR Algorithm } \\
\text { Rank-1 } \\
\text { Identification Accuracy }\end{array}$ & $\begin{array}{l}\text { Intra-Spec } \\
30 \text { vs. } 60 \mathrm{~m} \\
50 \%\end{array}$ & $\begin{array}{l}\text { al (NIR vs. } \\
30 \text { vs. 90m } \\
50 \%\end{array}$ & $\begin{array}{l}\text { SS-Distance Matching } \\
30 \text { vs. } 120 \mathrm{~m} \\
50 \% \\
\end{array}$ \\
\hline $\begin{array}{c}\text { AFIES } \\
\text { Bayesian MAP } \\
\text { Bayesian ML } \\
\text { LDA Euclidean } \\
\text { LDA lda_Soft } \\
\text { PCA Euclidean } \\
\text { PCA MahaCosine }\end{array}$ & $\begin{array}{l}0.85 \\
0.86 \\
0.88 \\
0.88 \\
0.68 \\
0.86\end{array}$ & $\begin{array}{l}0.52 \\
0.60 \\
0.61 \\
0.64 \\
0.27 \\
0.68\end{array}$ & $\begin{array}{l}0.36 \\
0.38 \\
0.38 \\
0.40 \\
0.20 \\
0.46\end{array}$ \\
\hline COTS & 0.16 & 0.02 & 0.01 \\
\hline $\begin{array}{c}\text { Developed Methods } \\
\text { MFSDF Without Mask } \\
\text { MFSDF With Mask } \\
\text { Fusion of MFSDF (best set) } \\
\text { With and Without Mask }\end{array}$ & $\begin{array}{l}0.89 \\
0.90\end{array}$ & $\begin{array}{l}0.66 \\
0.68\end{array}$ & $\begin{array}{l}0.55 \\
0.54 \\
\mathbf{0 . 6 4}\end{array}$ \\
\hline
\end{tabular}

recognition performance independent of whether operating at $30 \mathrm{~m}$ or $120 \mathrm{~m}$ standoff distance.

- Randomly selected 20,40,60\% of the images for training and the rest images were used for testing.

- This process was repeated 10 times.

The identification performance results of intra-spectral experiments are summarized in Table 5.2. Based on the results of the baseline experiments, it was determined that with academic software the identification rate was $100 \%$ at Rank-1 (as represented in Table 5.2). The results from AFIES outperformed the COTS software, where the identification rate is $37 \%$ for $90 \mathrm{~m}$ and $59 \%$ for $120 m$.

Intra-Spectral and Cross-Distance Face Matching: In this experiment, compared crossdistance face images, i.e. the baseline NIR face images $(30 \mathrm{~m})$ were compared to NIR images captured from $60 \mathrm{~m}$ to $120 \mathrm{~m}$ :

- $30 m$ vs. $60 m$

- $30 m$ vs. $90 m$ 
- $30 m$ vs. $120 m$

The intra-spectral, cross-distance experiments are summarized in Table. 5.3, Fig. 5.1 and Fig. 5.2 .

Based on the results, it is determined that developed method and AFIES achieve similar identification results for $N I R 30 \mathrm{~m}$ gallery matched against $N I R 60 \mathrm{~m}$ probe. The identification rank-1 accuracy is $88 \%$ for AFIES (LDA-EUC and LDA lda-soft) and $93 \%$ from the developed method (fusion of mask and non-mask). On the other hand, identification results are very low using COTS software, where the identification rank-1 accuracy is $16 \%$.

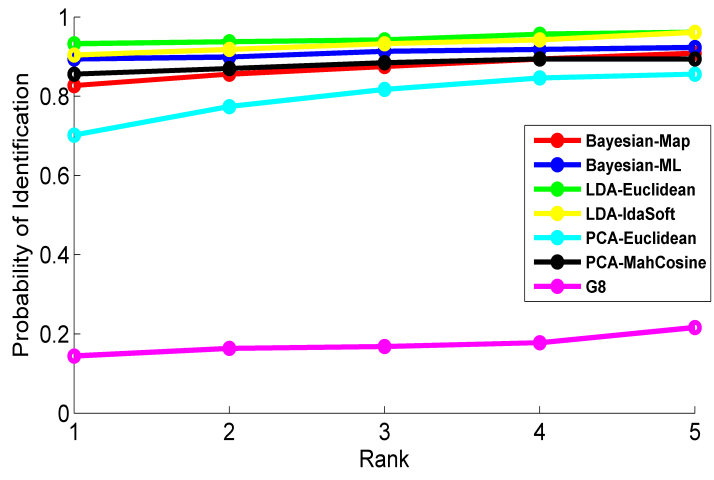

(a) AFIES

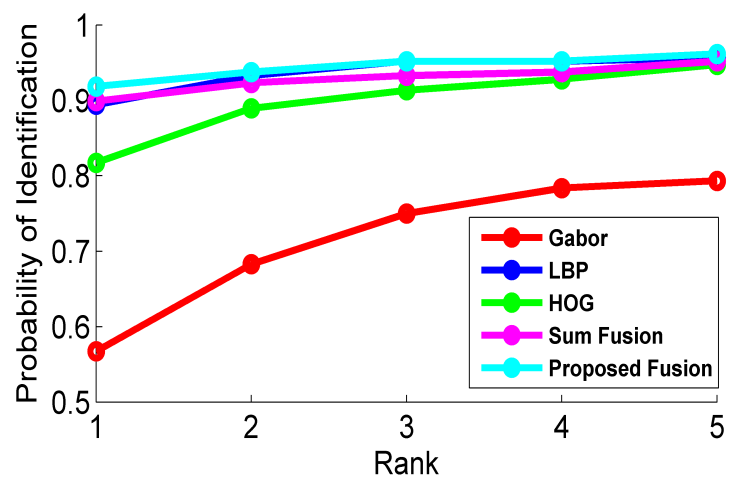

(c) Developed Without Mask.

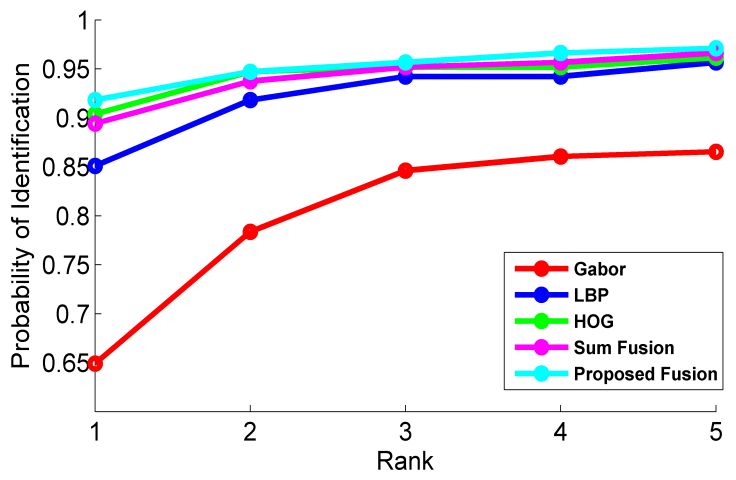

(b) Developed With Mask

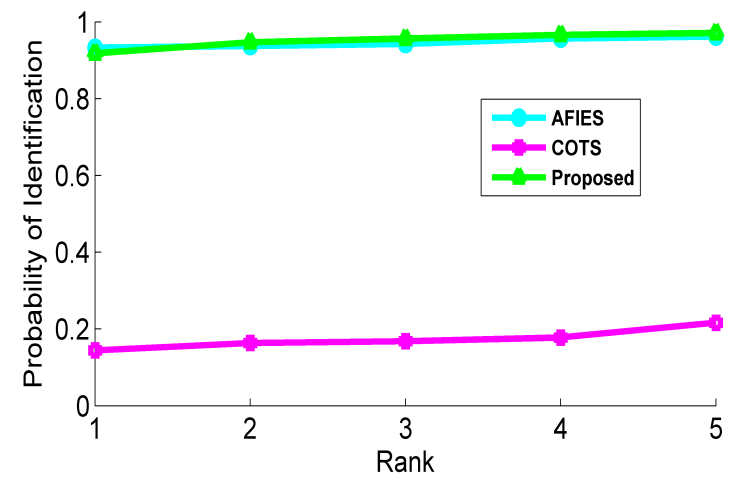

(d) AFIES and COTS vs. Developed.

Figure 5.1: Intra-spectral, cross-distance matching scenarios for NIR 30m (Gallery) against NIR 60m (Probe): Performance of baseline face recognition and developed MFSDF systems for cross-distance images without demographic filtering. Each algorithm was run 4 times and here the rank identification rate is presented for the best performing set.

For both $90 \mathrm{~m}$ and $120 \mathrm{~m}$ NIR probe images, a significant improvement is achieved from developed approach compared to AFIES. For example for $90 m$, the identification rank-1 accuracy is $81 \%$ (fusion of mask and no mask) from the developed method, whereas accuracy 


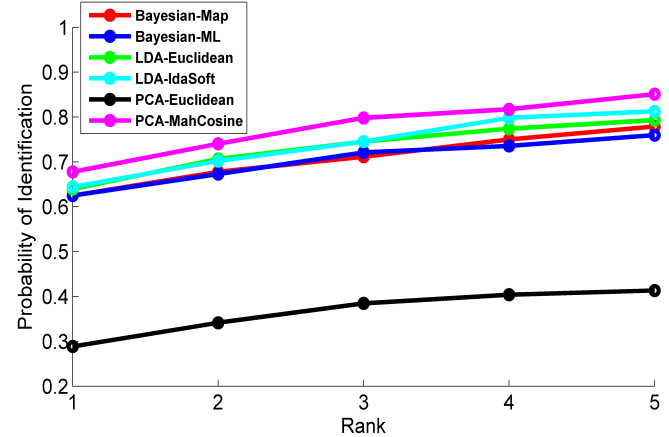

(a) AFIES Face Recognition System

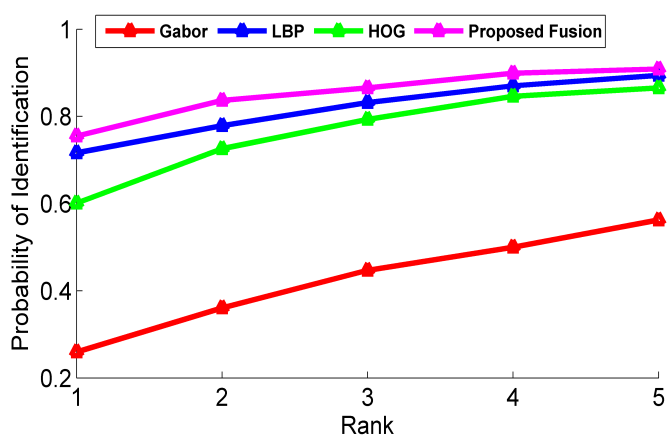

(c) Developed With Mask

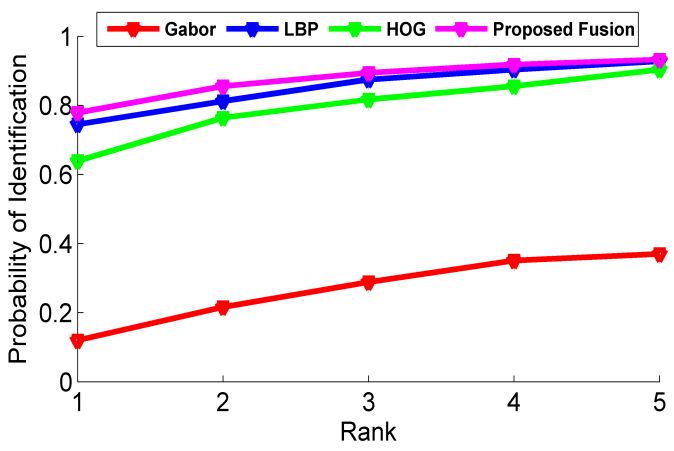

(e) Developed Without Mask

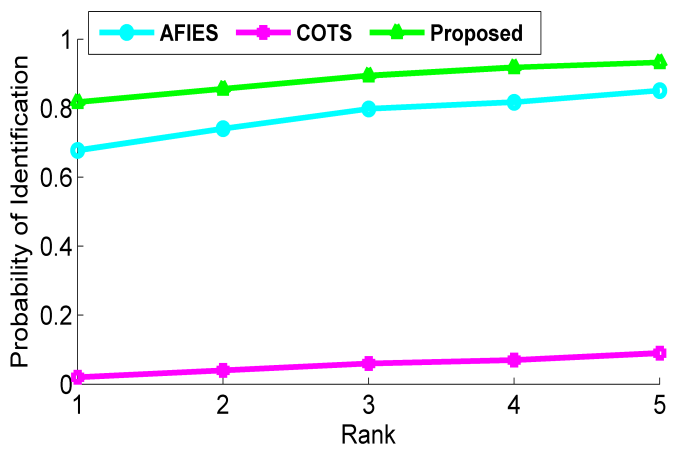

(g) AFIES and COTS vs. Developed

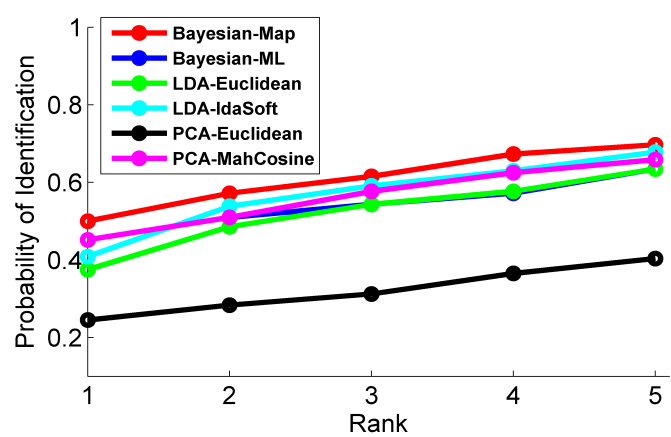

(b) AFIES Face Recognition System

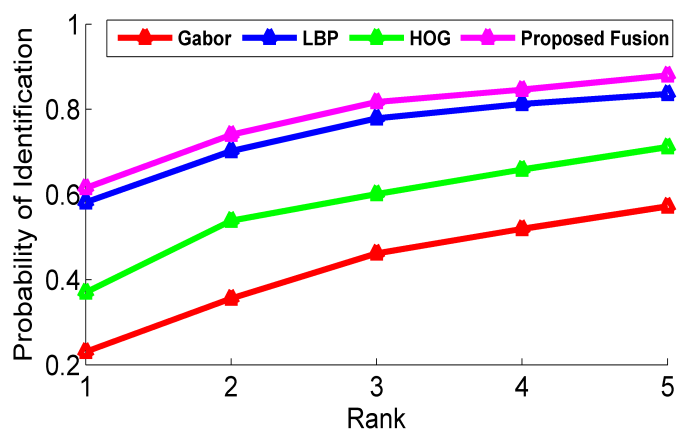

(d) Developed With Mask

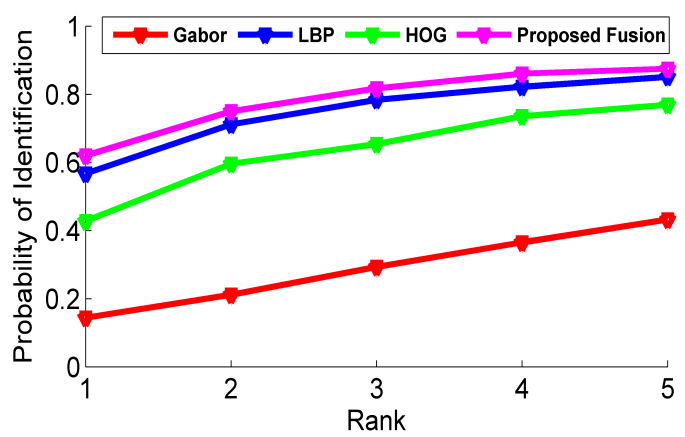

(f) Developed Without Mask

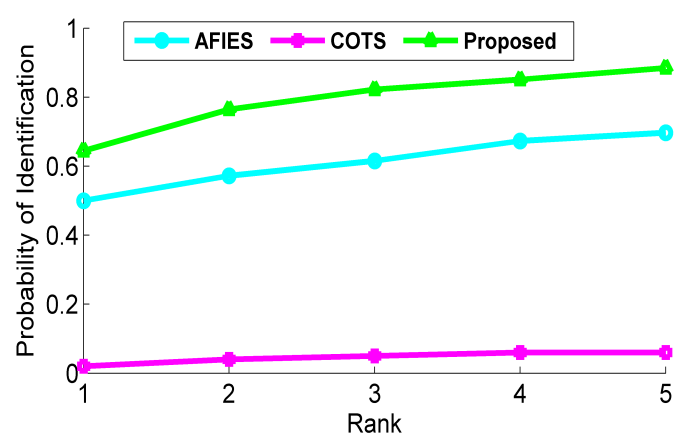

(h) AFIES and COTS vs. Developed

Figure 5.2: Intra-spectral, cross-distance matching scenarios for NIR 30m against NIR 90m (Left) and NIR 30m against NIR 120m (Right) without demographic filtering: Each algorithm was run 4 times and here the rank-1 identification accuracy is presented from best set. 
Table 5.4: In this table the cross-spectral, cross-distance matching scenarios (without demographic filtering) are investigated. Experimental results are presented when using $50 \%$ of the data for training and the rest data for testing. There is no overlap of subjects between training and testing. Experiments are run 4 times and here the rank-1 identification accuracy is presented.

\begin{tabular}{|c|c|c|c|c|}
\hline & \multicolumn{3}{|c|}{ Cross-Spectral (VIS vs. NIR), Cross-Distance Matching (Rank-1) } \\
Gallery: VIS 1m vs. & NIR 30m & NIR 60m & NIR 90m & NIRm \\
Rank-1 Identification Accuracy & $\mathbf{5 0 \%}$ & $\mathbf{5 0 \%}$ & $\mathbf{5 0 \%}$ & $\mathbf{5 0 \%}$ \\
\hline \hline AFIES & & & & \\
Bayesian MAP & 0.19 & 0.15 & 0.21 & 0.15 \\
Bayesian ML & 0.18 & 0.16 & 0.23 & 0.13 \\
LDA Euclidean & 0.19 & 0.20 & 0.13 & 0.11 \\
LDA lda_Soft & 0.19 & 0.22 & 0.13 & 0.12 \\
PCA Euclidean & 0.08 & 0.08 & 0.07 & 0.08 \\
PCA MahaCosine & 0.12 & 0.10 & 0.09 & 0.01 \\
\hline \hline COTS & 0.48 & 0.03 & 0.02 & 0.01 \\
\hline \hline Developed Methods & & & & \\
MFSDF Without Mask & 0.56 & 0.34 & 0.21 & 0.16 \\
MFSDF With Mask & 0.50 & 0.37 & 0.23 & 0.16 \\
\hline \hline
\end{tabular}

is only $68 \%$ from AFIES (PCA MahaCosine). For $120 m$, AFIES achieves $46 \%$ identification rate, while the developed method achieves $64 \%$ rank-1 accuracy.

The CMC curves for first 5 ranks, for the best performed set, out of randomly selected 4 different sets, are represented in Figs. 5.1 and 5.2. The identification accuracy is $77 \%$ for without mask, $75 \%$ for with mask and $81 \%$ for fusion of mask and non mask for the $90 m$ (see Fig. 5.2 and $64 \%$ for fusion of mask and no mask method for $120 \mathrm{~m}$ probe.

Cross-Spectral and Cross Distance Face Matching: In this, following visible to NIR face images are compared:

- VIS $1.5 m$ vs. NIR 30m

- VIS $1.5 m$ vs. NIR $60 m$

- VIS $1.5 m$ vs. NIR 90m

- VIS $1.5 m$ vs. NIR $120 m$

For cross-spectral, experiments are summarized in Table 5.4 and Fig. 5.3 (VIS vs. NIR 30m 
and VIS vs. NIR 60m). In all tables the mean rank-1 identification rates when using AFIES, COTS and developed MFSDF system are presented.

Based on the results, it is determined that the developed method achieves better performance results compared to AFIES and COTS system. For gallery at $30 \mathrm{~m}$ and probe at $60 \mathrm{~m}$, the identification rank-1 accuracy is 56\% (without mask) from the developed method, 19\% from AFIES and $48 \%$ from COTS. There is significantly better performance from developed approach over the academic and commercial method for $60 \mathrm{~m}$ and $90 \mathrm{~m}$ distance. For $60 \mathrm{~m}$, the identification accuracy is 37\% for developed MFSDF, $22 \%$ for AFIES and 3\% from COTS. For $90 m$, the identification accuracy is $23 \%$ for developed MFSDF and AFIES and $2 \%$ from COTS. For $120 m$, results are similar between developed and AFIES (15\%) and very low performance results are from COTS.

The CMC curves for first 5 ranks when compared visible to NIR face images captured at $30 \mathrm{~m}$, for the best performing set out of randomly selected 4 different sets, are presented in Fig. 5.3. The identification accuracies are 58\% for without mask, 52\% for with mask from developed system, $21 \%$ for AFIES and 51\% for COTS.

COTS provided us with good results only for one case, where the gallery images are matched against NIR 30m. The main reason for better performance of commercial system is that images are still with good quality. In this work, the database under un-controlled conditions at long distances (for example at $60 \mathrm{~m}, 90 \mathrm{~m}$ and $120 \mathrm{~m}$ ) is collected. The poor quality of the images (at long distances) results in poor performance of the COTS.

In order to examine the effectiveness of FR algorithms, each experiment is repeated 4 times for cross-scenarios using academic, commercial and developed face recognition methods. To see the performance variation between three FR matchers first the boxplots are plotted. In Fig 5.4, where the first 2 figures are for cross distance, and rest 4 figures are for cross spectral face matching.

In this the identification rank-1 accuracy from developed method is compared with AFIES. COTS performed well only for VIS $1 \mathrm{~m} v \mathrm{~s}$. NIR $30 \mathrm{~m}$ and accuracy is very low for rest of the 7 sets when gallery data (NIR 30m, VIS Im) is compared against probe data $(60 \mathrm{~m}, 90 \mathrm{~m}, 120 \mathrm{~m})$ as represented in Table 5.3 for cross distance and in Table 5.4 for cross spectral experiments. 


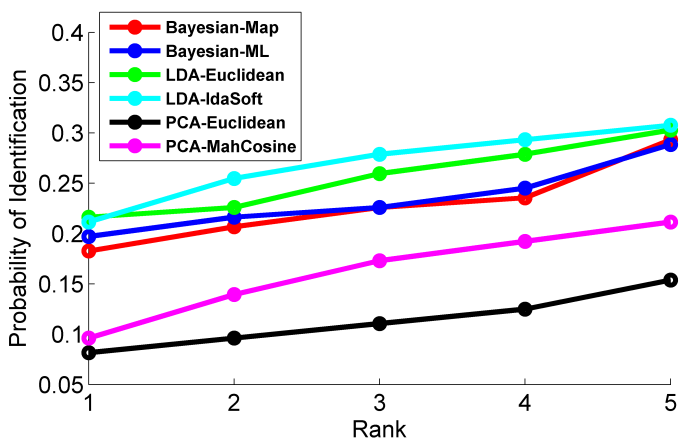

(a) AFIES Face Recognition System

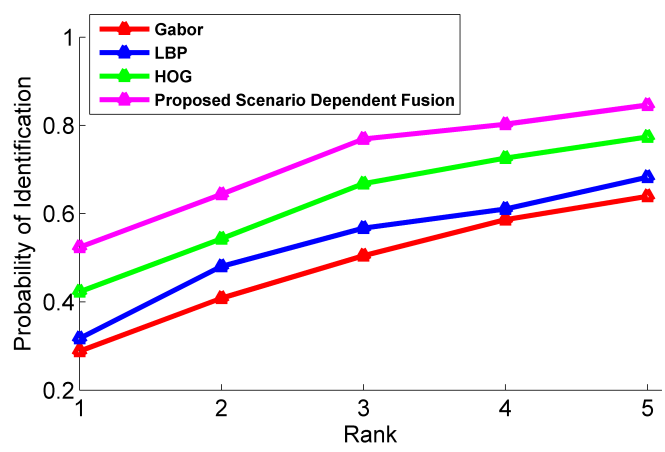

(c) Developed With Mask

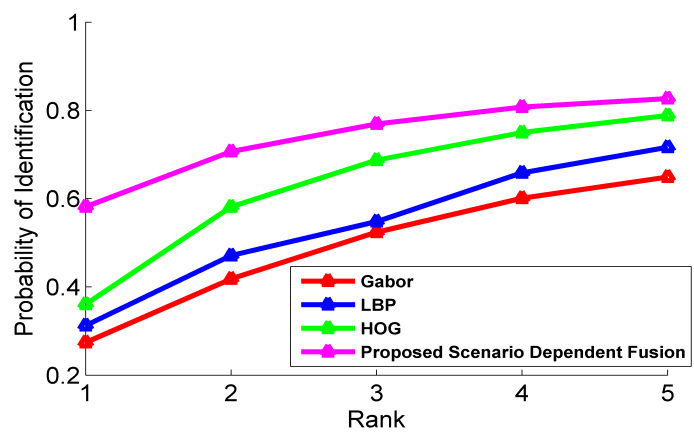

(e) Developed Without Mask

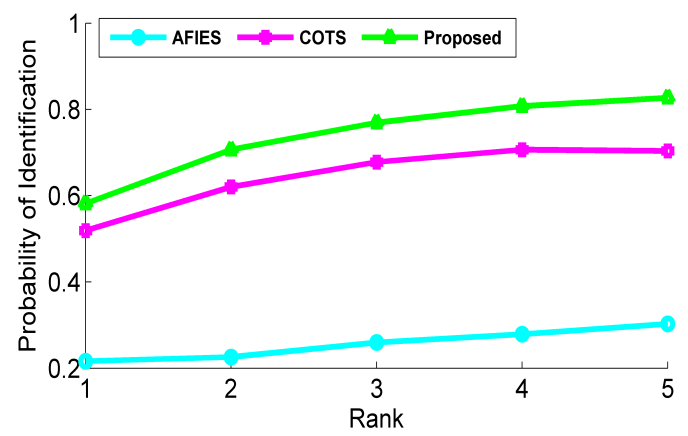

(g) AFIES and COTS vs. Developed

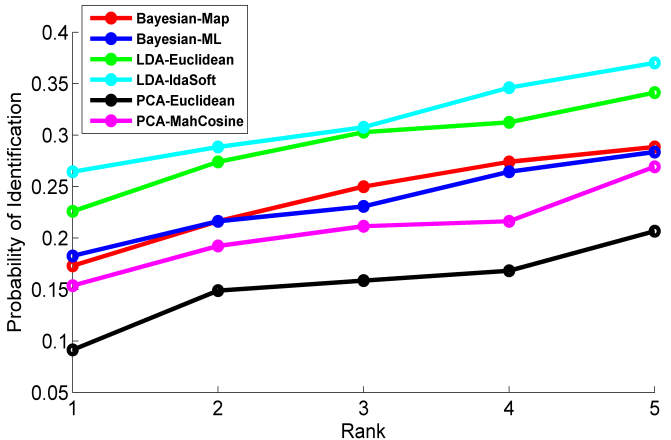

(b) AFIES Face Recognition System

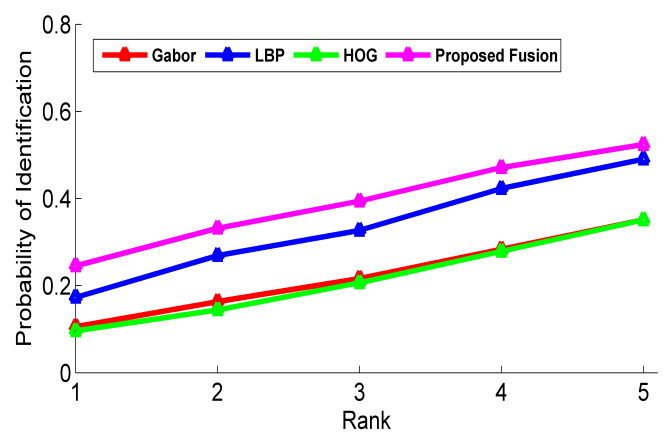

(d) Developed With Mask

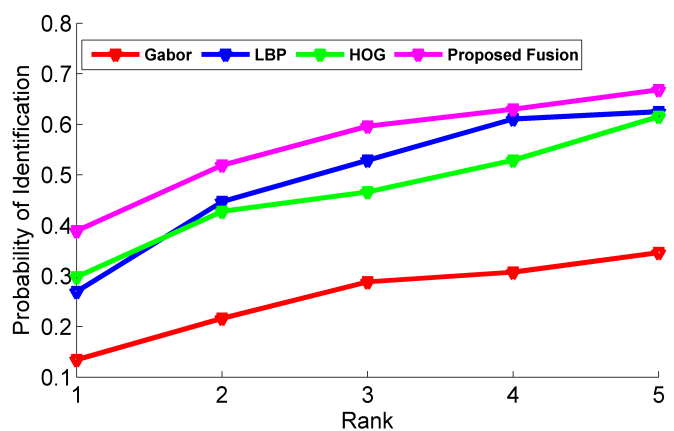

(f) Developed Without Mask

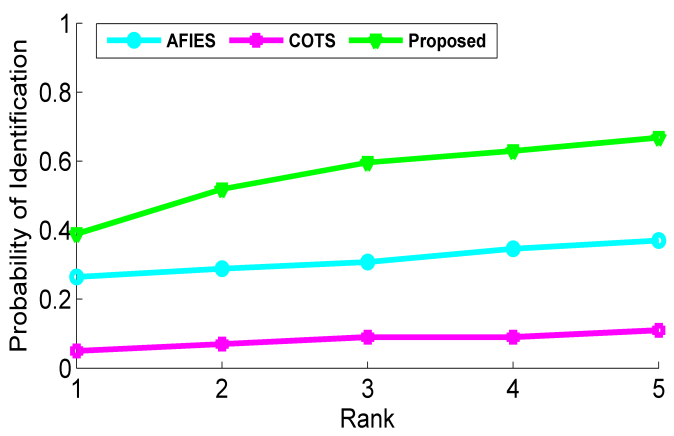

(h) AFIES and COTS vs. Developed

Figure 5.3: Cross-spectral, cross-distance matching scenarios for VIS $1 \mathrm{~m}$ (Gallery) against NIR 30m (Probe) (Left) and VIS $1 m$ (Gallery) against NIR 60m (Probe) (Right). 


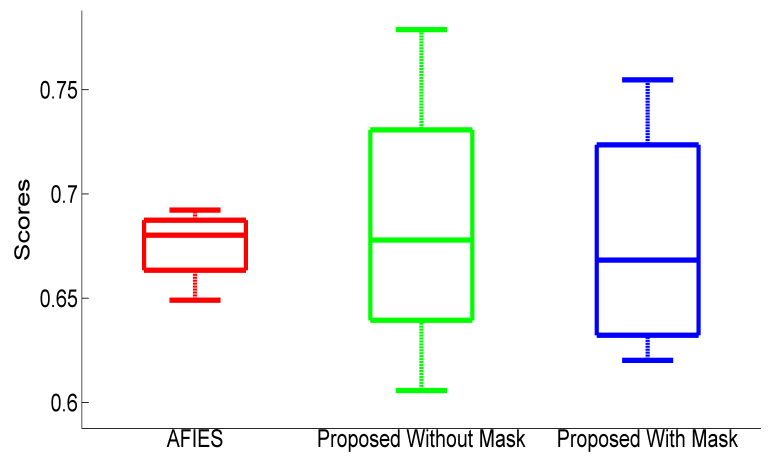

(a) NIR 30m vs. NIR 90m

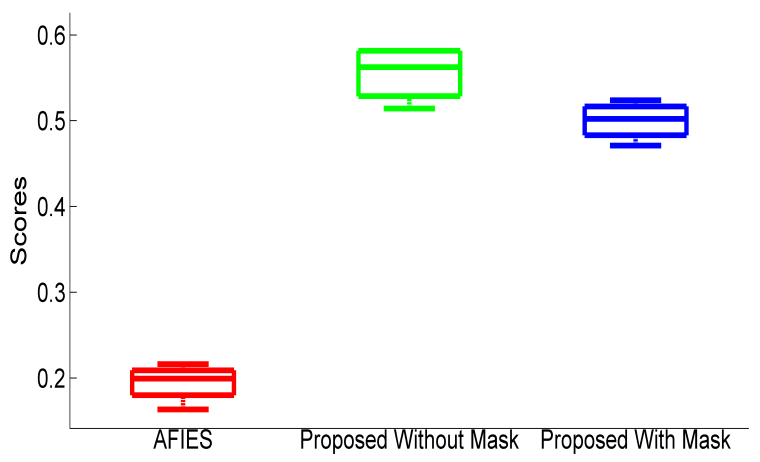

(c) VIS Im vs. NIR 30m

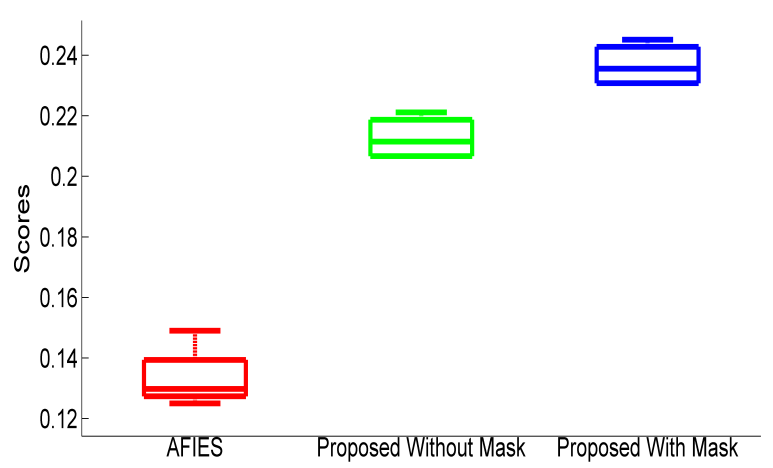

(e) VIS Im vs. NIR $90 m$

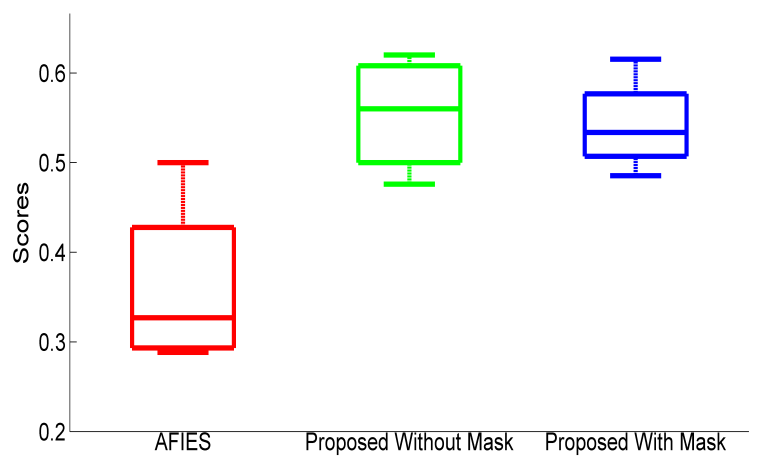

(b) NIR 30m vs. NIR $120 \mathrm{~m}$

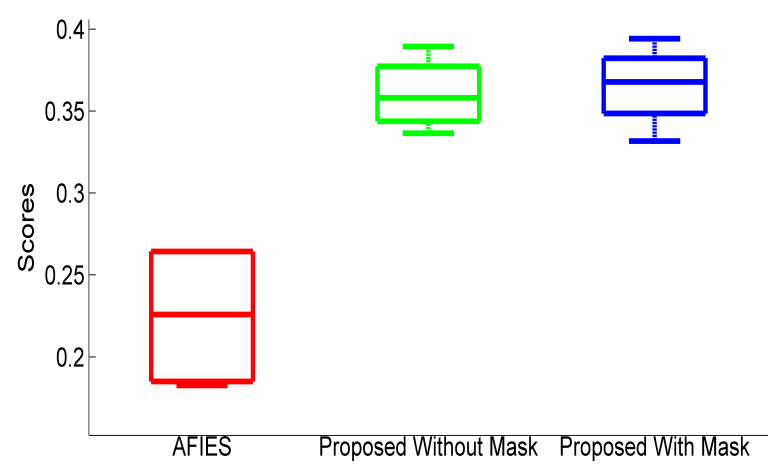

(d) VIS Im vs. NIR 60m

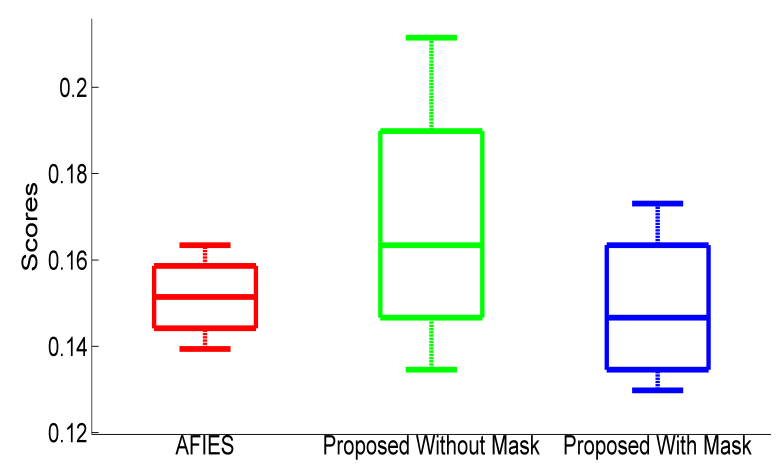

(f) VIS Im vs. NIR $120 \mathrm{~m}$

Figure 5.4: Box-plots for cross-distance using intra-spectral and cross-spectral matching scenarios after running each experiment 4 times.

Based on the mean and variance of boxplots, the matching accuracy from developed method (using either with or without mask), for NIR 30m vs. NIR 90m, is overall higher for developed method compared to AFIES. For NIR 30m vs. NIR 120m and from cross-spectral (all the 4 cases c, d, e and f), the developed method outperformed AFIES. 


\subsection{Conclusion from Study 1 for NIR Database}

\section{-Intra-spectral and Cross-Distance Rank-1 Identification Accuracy:}

- $30 \mathrm{~m}$ vs. $60 \mathrm{~m}$ : $16 \%$ using COTS, $88 \%$ using AFEIS to $93 \%$ using developed.

- $30 m$ vs. $90 m$ : $2 \%$ using COTS, $64 \%$ using AFEIS to $68 \%$ using developed.

- $30 m$ vs. $120 m$ : $1 \%$ using COTS, $40 \%$ using AFEIS to $64 \%$ using developed.

\section{- Cross-Spectral and Cross-Distance Rank-1 Identification Accuracy:}

- VIS 1.5 m vs. NIR 30m: $19 \%$ using COTS, $48 \%$ using AFEIS to 56\% using developed.

- VIS 1.5 m vs. NIR 60m: 3\% using COTS, 22\% using AFEIS to 37\% using developed.

- VIS 1.5 m vs. NIR 90m: 2\% using COTS, to $23 \%$ using AFEIS and the developed method.

- VIS $1.5 \mathrm{~m}$ vs. NIR 120m: 1\% using COTS, to 16\% using AFEIS and the developed method.

\subsection{Threats to Validity}

Campbell and Stanley [89] define two types of validities; internal and external. Cook and Campbell [90] extended the list to four types; internal, external, construct and conclusion. Problems that cause our representations to be wrong are defined as threats to validity. The validity is threatened either by poor theory or poor measurement [91, 92]. The basic principle of causality (cause and effect) determines whether the results and trends seen in experiments are actually caused by manipulations or whether from some other factor or confounding factor. The possible threats to this framework are discussed below:

\subsubsection{Construct Validity}

This validity concerns how well the proposed framework or theories are implemented into actual programs [93]. It is concerned with the relationship between the theory and observation 
[94]. If we are not building the right system, nothing else matters [89].

\section{Threats from Developed Study:}

- To construct an efficient face recognition system in this work, video sequences of fullfrontal faces are collected in visible and NIR band from the designed night time imagery system at fixed standoff distances. During data collection, change in the light conditions, environment conditions, face pose, and camera focus can affect the performance of face recognition system. These factors are defined as confounding factors and responsible for variation in the performance. For the data collection at long standoff distances, occluded faces from vehicles on the road, trees, and other subjects act as confounding factor.

- Developed approach is implemented using LBP, Gabor and HOG descriptors. If researchers want to add a new descriptor to the system, they need to modify the algorithm, otherwise the accuracy of the system will be affected.

- Developed approach is implemented using score level fusion scheme. If users want to add a new fusion scheme to the system, for example based on sensor level, decision level or feature level schemes, they need to modify the algorithm, otherwise the accuracy of the system will be affected.

\subsubsection{Internal Validity}

It is a measure which ensures that experimental design closely follows principal of cause and effect [95]. There is no third factor or confounding factor. There are some parameters that may affect the experimental finding. In this work, the internal validity is investigated based on parameters like training size, image window size and number of samples.

\section{Threats from Developed Study:}

- Performance of the developed face matching approach is dependent on the size of normalized image (using image registration). To use the new datasets, researchers need to normalize the face images, otherwise it may result in poor performance.

- Performance of the developed face matching approach is dependent on the training data 
set (Visible and NIR band). Hence, to use the new datasets (Face database in SWIR and Thermal band), researchers need to retrain the system.

\subsubsection{Conclusion Validity}

This validity is concerned with the relationship between the treatment and the outcome [96]. The developed system is reliable, achieved output results are same when the same set of experiments are repeated using random training and testing sets.

\section{Threats from Developed Study:}

- Face matching algorithm is working well for academic database with 103 subjects. In a scenario, where the researchers want to train the system on new database, they need to consider sufficient amount of database to train the system, otherwise the performance of the system may be affected from small sample size.

\subsubsection{External Validity}

This validity is concerned about generalization. The main concern is up to what level or point a result or outcome can be generalized to population, treatment variables and settings.

\section{Threats from Developed Study:}

- The threat may be caused if the developed system performs well only for a specific database and not for the unseen database. In this study, we tested the developed system on LDHF database collected under un-constrained conditions. Based on the results, it is concluded that the developed system performed well for other existing databases. However, to make the system robust it needs to be tested on other databases which could not be accessed. 


\section{Chapter 6}

\section{CNN based Demographic Filtering for}

\section{Night Time Long Range Imagery System}

\section{in NIR Band}

As discussed in Chapter 3, the main challenge for the NIR database collected under challenging conditions is the automatic demographic filtering of the data in terms of gender (male and female) and ethnicity (Asian and Caucasian) class. To address this problem, a deep learning based method for the demographic filtering is developed. The focus of this chapter is to discuss implementation of the developed convolutional neural network for demographic filtering from Study 2 and to determine whether the usage of demographic filtering can enhance recognition performance [69]. The architecture for this study is represented in Fig. 6.1

\subsection{Demographic Filtering from developed CNN Architec- ture}

\subsubsection{Training and Testing}

In the experiments performed in this study, the subjects in the training and test sets are different and the images are taken at different locations and days. CNN based gender and 


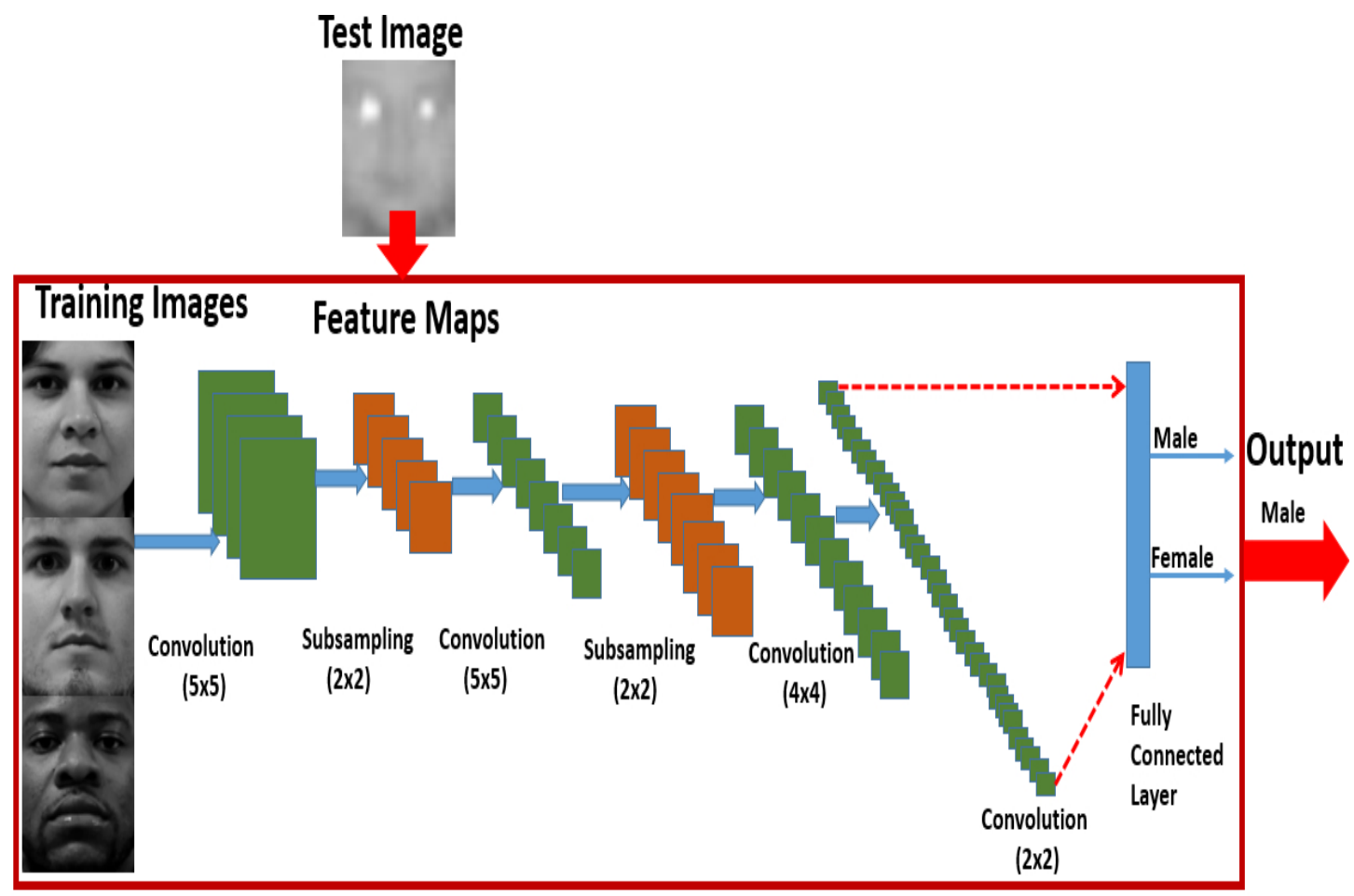

Figure 6.1: An overview of the developed $\mathrm{CNN}$ architecture.

ethnicity stratification approach is developed. This work is extended further to classify the gender class with the most significant attributes with in the class. For the male class, the facial hair as the most discriminative attribute are selected and grouped into male with or without beard. The female class is further grouped into female Asian or Caucasian.

Training Data: For the limited amount of training data, CNN pre-trained on large databases (ImageNets) is used by the researchers for the recognition and classification tasks. In this work, to collect a large training database from available image repositories and to label the database manually is time consuming process. There was no pre-trained multi-sensor network model available to use for $\mathrm{CNN}$ network, therefore in this work the models are trained for the original database.

To train the CNN network, two sets of experiments are performed:

- Experiment 1, where LDHF database [29, 31] is selected for the training and our database for the testing part.

- Experiment 2, 9 different databases are selected for the training and our database is se- 
lected for the testing.

\subsubsection{Ethnicity Class}

The two sets of experiments are conducted as follows:

\section{Experiment 1:}

- Scenario 1 , there is an overlap between the subjects for training and testing, the experiments are performed using our WVU database (VIS-VIS, VIS-NIR). The VIS face images at short distance of $1 \mathrm{~m}$ are selected to train the CNN network and the NIR face images, collected at long distances, for the testing. To perform the stratification experiments, $50 \%$ of the data (subjects) is selected to train the system and the rest for the testing. This process is repeated 4 times, where for each set the different training and testing data is selected.

- Scenario 2, the LDHF database is selected and extended the training data set by using also the WVU database. For testing, the WVU database collected in NIR band at long standoff distances is selected. To perform the stratification experiments, the data from the LDHF database and 50\% from WVU is selected to train the system. The rest of the data is selected for testing with no overlap of subjects.

Problem With Scenario 2: For the training data (WVU database + LDHF), the LDHF database consists of primarily Asian population data. To deal with this issue 9 different types of databases are selected.

\section{Experiment 2:}

To train the system, 9 different types of databases collected indoors, outdoors, with different camera sensors, expressions, ethnicity, locations and times are selected.

Testing Data: To evaluate the performance, database collected in our lab in visible band at short distance $(1.5 \mathrm{~m})$ and NIR face database collected in night time at long stand-off distances of $30 \mathrm{~m}$ to $120 \mathrm{~m}$ is selected. For the case when both the training and testing data are selected from same database, which is far from the realistic scenario. In this work, two scenarios are selected to perform the stratification: 
- Scenario 1: In this all the 9 databases including, WVU visible-thermal profile face (VTPF) database [97], WVUM database [98], Tinders Database [17], QFire Database, FEI face database [99], Libor Spacek Facial database [100] are selected to train the system and our database for the testing part.

- Scenario 2: $25 \%$ of our (VIS $1.5 \mathrm{~m}$ ) database and all the 9 databases are selected to train the system, where the rest of our database (VIS $1.5 \mathrm{~m}$ and NIR 30m to $120 \mathrm{~m}$ ) is selected for the testing without any overlap of the subjects.

\subsubsection{Gender Class}

- Scenario 1, where the LDHF face images in NIR band at a distance of $1 \mathrm{~m}$ are used for training and the WVU database (NIR dataset $30 \mathrm{~m}$ up to $120 \mathrm{~m}$ ) is selected for testing.

- Scenario 2, the VIS face images from the LDHF database are selected for training and the NIR dataset from the WVU database is selected for testing.

- Scenario 3, the VIS face images at a short distance from both LDHF and WVU databases are used for training, while the WVU database at long standoff distances is used for testing. There is no overlap of subjects for the training and testing. All the database from the LDHF (70 males and 30 females) and $20 \%$ of the data (subjects) from the WVU is selected to train the system. The rest of the $80 \%$ of the data (subjects) from the WVU is selected for testing. This process is repeated twice, where for each set the different training and testing data is selected.

In all these scenarios, training and test sets consist of images from different subjects, sensors, light conditions and locations. For experiment 2, the same set of databases are selected which are used for the Ethnicity class with two scenarios.

\subsubsection{Optimization}

An empirical optimization on epoch and momentum parameters is conducted that resulted in better ethnicity and gender based stratification accuracy. 
Training Data: VIS Images, Testing Data: VIS and NIR Images

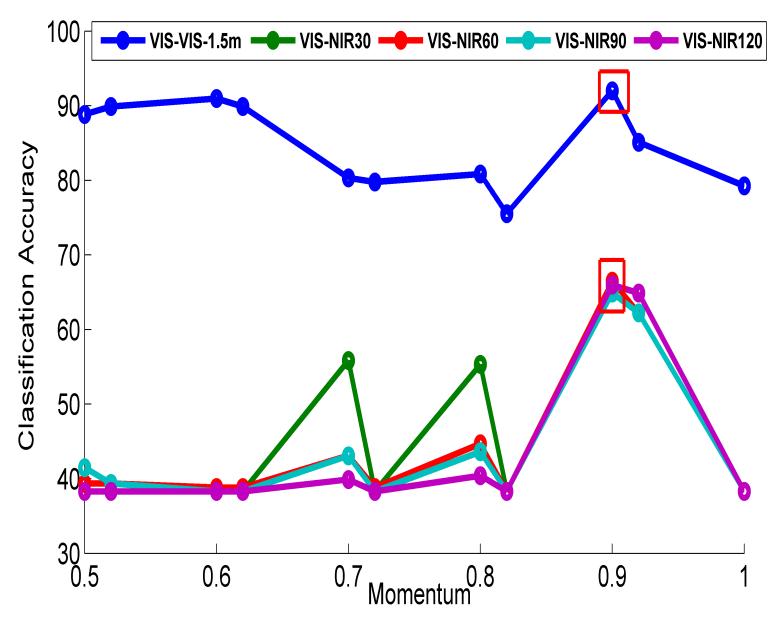

(a) Ethnicity Class

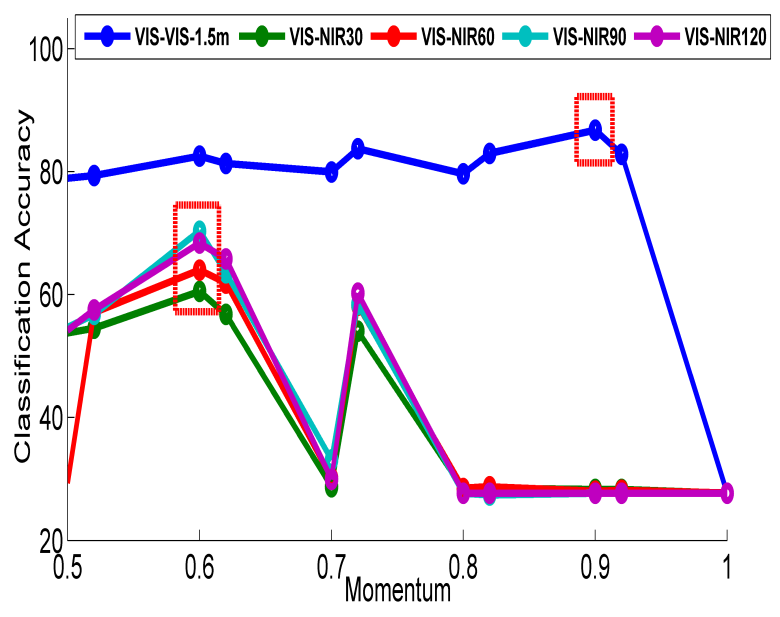

(b) Gender Class

Figure 6.2: Selection of Momentum for the Ethnicity and Gender Class. For intra-spectral stratification, visible images were used for training and testing. For cross-spectral stratification, visible images (VIS $1.5 \mathrm{~m}$ ) were used for training (to simulate the most challenging scenario of having a gallery set composed of only visible face images) and NIR images collected at a distance of $30 \mathrm{~m}, 60 \mathrm{~m}, 90 \mathrm{~m}$ and $120 \mathrm{~m}$ distance were used for the testing.

Selection of Epoch: A series of experiments are performed with the selected epoch values of $4,8, \ldots ., 52$. Based on the results, the best stratification or demographic filtering results are achieved for epoch value 16 for ethnicity class. The same set of experiments is performed for the gender class and an epoch value of 16 is selected.

Selection of Momentum: Based on [79], the momentum values lie in the range from 0.50 to 1. To select the best value, a series of experiments are performed with values of $0.50,0.52$, $0.54, \ldots, 1$. The experiments are conducted for both the ethnicity and gender classes. As shown in Fig. 6.2 for the ethnicity class the highest stratification accuracy is achieved when the momentum value is 0.90 . For the gender class, the highest stratification accuracy is achieved when the momentum value is 0.60 for NIR (30m up to $120 \mathrm{~m}$ ) and 0.90 for the VIS band in the testing set as shown in Fig. 6.2

In the first set of experiments, experiments aim to illustrate how the scenario adaptable deep learning system performs for intra-spectral band, where both the training and testing data is selected from the same band (VIS-VIS) under controlled conditions. Finally, cross-spectral experiments are performed in order to determine the extent of which the performance of the developed system to perform the demographic filtering or data stratification is affected when 
the standoff distance increases, when the face images from visible band (short distance) are selected for the training and NIR face images from a distance of $30 \mathrm{~m}$ up to $120 \mathrm{~m}$ for testing. To train the CNN network two scenarios are selected.

\subsubsection{Demographic Filtering Results for Ethnicity Class}

For the ethnicity class, from a set of values selected in range from 0.50 to 1 , the highest stratification accuracy results are achieved when the momentum value is 0.90 (for $1.5 \mathrm{~m} u p$ to $120 \mathrm{~m}$ ) as shown in Fig. 6.2. For model 1, stratification is performed where the network is trained when the momentum value is 0.90 and epoch value is 16 . The stratification accuracy is more than $85 \%$ for the Caucasian group. For model 2 (with a different set of learning parameters), the stratification accuracy is more than $75 \%$ for the Asian group. Finally, a significantly better improvement in the performance results is achieved, when combined the model 1 and model 2. The overall accuracy is almost $95 \%$ as shown in Table 6.1 for cross-spectral demographic filtering (VIS-NIR 30m).

Training Data: VIS Images, Testing Data: VIS and NIR Images

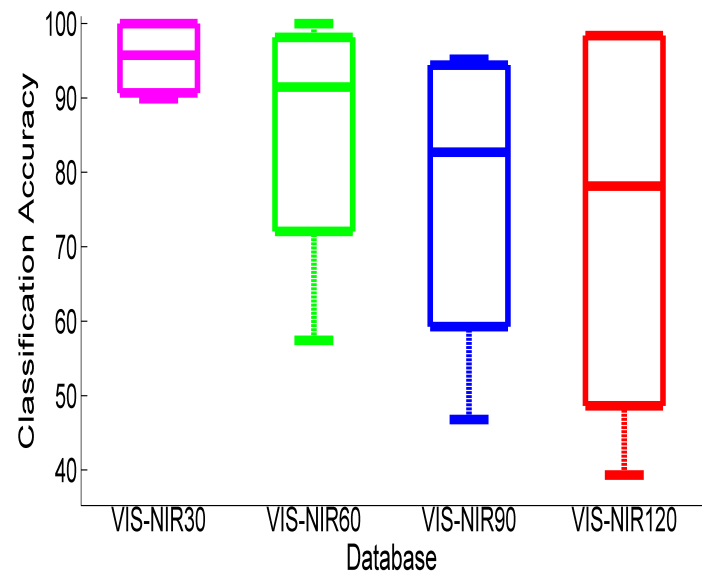

(a) Scenario 1

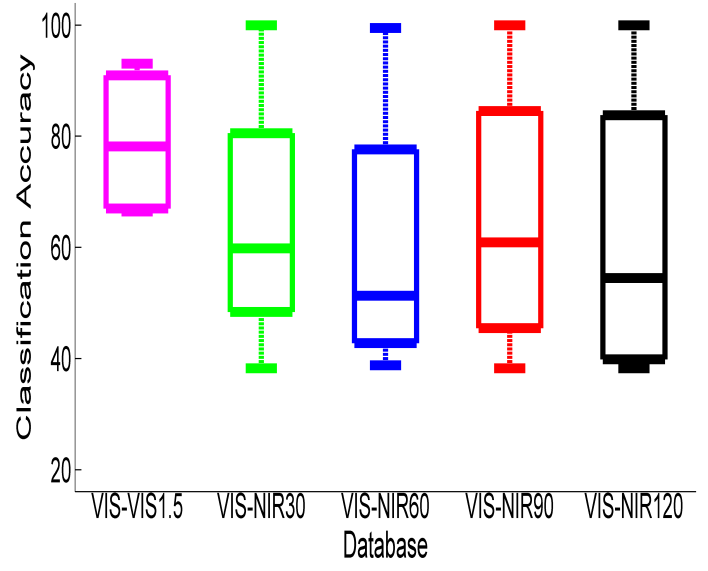

(b) Scenario 2

Figure 6.3: Demographic filtering results for the ethnicity class. Scenario 1 (Left), is based on overlapping of subjects between training and testing. Scenario 2 (Right), is based on no overlapping of subjects between training and testing.

In order to examine the effectiveness of $\mathrm{CNN}$ based demographic filtering system (trained on parameters selected from optimization), each experiment is repeated 4 different times, where each time a different training set was randomly selected and rest of the data was used for testing 
Table 6.1: Summary of stratification (demographic filtering) results for the ethnicity class based on $\mathrm{CNN}$ for each dataset. To perform CNN, the model is trained for the challenging testing database. WVU database consists of 103 subjects and LDHF database consists of 100 subjects. Scenario 1, WVU (50\%) database for training and the rest of the database for testing. Scenario 2, LDHF (ALL) and WVU (50\%) database for training and the rest of the database for testing.

\begin{tabular}{|c|c|c|}
\hline & \multicolumn{2}{|c|}{ Demographic Filtering Accuracy (\%) } \\
\hline $\begin{array}{c}\text { Datasets } \\
\text { Train-Test }\end{array}$ & Scenario 1 & Scenario 2 \\
\hline VIS-VIS 1.5m & 99.04 & 78.98 \\
\hline VIS-NIR 30m & 95.34 & 64.49 \\
\hline VIS-NIR 60m & 85.10 & 60.23 \\
\hline VIS-NIR 90m & 76.86 & 65.02 \\
\hline VIS-NIR 120m & 73.53 & 61.83 \\
\hline
\end{tabular}

(Scenario 1 and Scenario 2). To see the performance variation in the stratification results with increasing distance for the testing set, the boxplots are plotted as shown in Fig. 6.3 (each boxplot is based on results from 4 randomly selected training and testing sets). Based on mean and variance plots, the better data stratification or demographic filtering results are achieved for testing data at distance of $30 \mathrm{~m}$ for Scenario 1.

Table 6.1 depicts the accuracy results for the ethnicity class from deep learning system. Based on the results, it is determined that (trained model on WVU database) for the ethnicity class for scenario 1, the stratification accuracy is $95 \%$ when the VIS dataset was used for training while the NIR 30m dataset for testing. The system achieves promising stratification performance results, when the NIR face images at a long standoff distance of $60 \mathrm{~m}, 90 \mathrm{~m}$ and $120 \mathrm{~m}$ are selected for testing. The decrease in the performance of the system for scenario 2 with the extended database is due to the variation in light conditions and sensors, under which the training images were collected.

In experiment 2, 9 databases are used to train the system. To perform the stratification, two scenarios are selected:

Scenario 1: In this total 9 databases are selected for training and our database is used for testing.

Scenario 2: Both our database and 9 other databases are used for the training and our database is used for the testing (without overlap of subjects). 
Table 6.2: Summary of stratification results for the Ethnicity class based on CNN for each dataset. To perform stratification, model is trained on 9 different databases. Scenario 1, 9 databases for training and WVU database for testing. Scenario 2, 9 other databases and WVU $25 \%$ database for the training and the rest of the WVU database for testing.

\begin{tabular}{|c|c|c|}
\hline \multicolumn{3}{|c|}{ Demographic Filtering Accuracy (\%) } \\
\hline \multicolumn{2}{|c|}{ Ethnicity Class: Scenario 1- Training with Extended Database) } \\
\hline \multicolumn{2}{|c|}{ Datasets: Train VIS (9 Databases) vs. Test (Own Database) } \\
\hline Parameters & CNN & BOW \\
\hline VIS 1.5m & 71.33 & 45.65 \\
\hline NIR 30m & 57.88 & 58.56 \\
\hline NIR 60m & 58.02 & 52.04 \\
\hline NIR 90m & 60.60 & 59.24 \\
\hline NIR 120m & 65.63 & 62.23 \\
\hline
\end{tabular}

For intra-spectral case, for both the scenarios 1 and 2, the face images collected in the visible band (at different locations, time, expressions and illumination conditions, expressions) are selected. The network is trained with fixed momentum value of 0.92 , learning rate of 0.02 and batch size of 100. The epoch value is selected in the range from $4-52$ with a interval of 4 and, finally, the values are selected where achieved the best stratification results. For crossspectral case, images collected in the visible band are selected to train the network and NIR images collected at a distance of $30 \mathrm{~m}$ to $120 \mathrm{~m}$ are selected for the testing. To compare the results with baseline system, the bag of words model is selected.

\subsubsection{Demographic Filtering Results for Gender Class}

- For Scenario 1, NIR images are used for training. For Scenario 2, VIS images are used for training. In both the scenarios, LDHF database is used for the training and WVU database is used for testing. For Scenario 2, the highest stratification accuracy achieved was $78 \%$, as presented in Table 6.3 . However, the results were not satisfactory $(32 \%$ accuracy) when the NIR images collected from long standoff distance were used.

- Scenario 3, the VIS band face images from both the LDHF and WVU databases are used for training, while the WVU database is used for testing. A significant improvement in the classification results is achieved with extended database used for training based on 
both the WVU and LDHF database.

The model parameters are selected for the values, where the highest stratification accuracy is achieved. For model 1, stratification is performed where the network is trained with the momentum value of 0.60 for the NIR class and 0.90 for the VIS class. The stratification accuracy is more than $85 \%$ for the female group. For model 2 (with different set of learning parameters), the stratification accuracy is more than $90 \%$ for the male group. Finally, a significantly better improvement in the performance results is achieved when combined the model 1 and model 2. The overall stratification or filtering accuracy is more than $95 \%$ as presented in Table 6.3 for intra-spectral stratification (VIS-VIS $1.5 \mathrm{~m}$ ).

Table 6.3: Summary of demographic filtering results for the gender class based on CNN for each dataset. To perform CNN, model is trained for the challenging testing database. Scenario 1, where NIR images are used for training. For Scenario 2, VIS images are used for training. In both the scenarios, LDHF database is used for the training and WVU database is used for testing. Scenario 3, the VIS band face images from both the LDHF and WVU databases are used for training, while the WVU database for testing.

\begin{tabular}{|c|c|c|c|}
\hline \multicolumn{4}{|c|}{ Demographic Filtering Accuracy (\%) } \\
\hline \multicolumn{4}{|c|}{ CNN based Stratification into Gender Class } \\
\hline Parameters & Scenario 1 & Scenario 2 & Scenario 3 \\
\hline VIS 1.5m & 31.92 & 78.28 & 96.41 \\
\hline NIR 30m & 32.40 & 32.65 & 86.14 \\
\hline NIR 60m & 32.40 & 32.52 & 89.45 \\
\hline NIR 90m & 30.95 & 32.04 & 93.52 \\
\hline NIR 120m & 32.16 & 32.04 & 94.12 \\
\hline
\end{tabular}

\subsubsection{Demographic Filtering Results for Male Class into With or Without}

\section{Beard}

In this, CNN network is developed for the grouping of the male class into with or without beard. Table 6.4 describes the accuracy results for the developed CNN architecture with best epoch value and baseline model.

- For Intra-spectral demographic filtering, the highest stratification accuracy achieved is $54 \%$ from scenario 1 as presented in Table 6.4 with epoch value of 4 from a range of 
epoch value from $(4,8, \ldots, 52)$.

- For Cross-spectral demographic filtering, the stratification accuracy is more than $60 \%$ for scenario 1, when the visible images are selected for the training and NIR images collected at a distance of $30 \mathrm{~m}$ for testing.

- VIS vs. NIR 60m, the stratification accuracy is more than $65 \%$ from scenario 2 and better results are achieved than the baseline model (BOW).

- VIS vs. NIR 90m, the stratification accuracy is more than $60 \%$ from scenario 2 and better results are achieved than the baseline model (BOW).

- VIS vs. NIR 120m, the stratification accuracy improved from $40 \%$ (the baseline BOW model) to $67 \%$ from developed CNN network.

Based on the results, it is concluded that for cross-spectral, better performance results are achieved from the developed CNN network for scenario 2. For intra-spectral, the better performance results are achieved from the baseline model (BOW) for scenario 1.

Table 6.4: Summary of demographic filtering results for the ethnicity and gender class based on $\mathrm{CNN}$ for each dataset. To perform $\mathrm{CNN}$, the model is trained for the challenging testing database.

\begin{tabular}{|c|c|c|c|c|c|}
\hline \multicolumn{6}{|c|}{ Demographic Filtering Accuracy (\%) for Male Class into With or Without Beard } \\
\hline \multicolumn{6}{|l|}{ Datasets: Train vs.Test } \\
\hline VIS vs. & VIS $1.5 m$ & NIR 30m & NIR 60m & NIR 90m & NIR 120m \\
\hline Scenario 1: CNN & 53.75 & 60.36 & 63.21 & 61.25 & 62.50 \\
\hline Scenario 1: BOW & 69.46 & 62.14 & 62.68 & 61.607 & 40.36 \\
\hline Scenario 2: CNN & 52.64 & 55.53 & 68.51 & 63.94 & 67.31 \\
\hline Scenario 2: BOW & 45.53 & 40.00 & 38.04 & 37.32 & 37.67 \\
\hline \multicolumn{6}{|c|}{ Demographic Filtering Accuracy for Female Class into Asian or Caucasian Class } \\
\hline Scenario 1: CNN & 74.24 & 66.67 & 57.95 & 57.58 & 64.01 \\
\hline Scenario 1: BOW & 75.75 & 56.06 & 55.68 & 56.06 & 57.58 \\
\hline Scenario 2: CNN & 71.12 & 71.00 & 68.50 & 64.00 & 60.00 \\
\hline Scenario 2: BOW & 36.00 & 59.50 & 66.00 & 58.00 & 62.00 \\
\hline
\end{tabular}




\subsubsection{Demographic Filtering Results for Female Class into Asian or Cau- casian}

In this, CNN network is developed for the grouping of the female class into Asian or Caucasian (intra-spectral and cross-spectral stratification). In Table 6.4, stratification results are presented with best epoch value. Based on the results, it is concluded that the best performance results for cross-spectral stratification are achieved from developed CNN network in comparison to the baseline BOW model.

- VIS-NIR 30m, the stratification accuracy reaches greater than $70 \%$ from developed CNN network for scenario 2. The stratification accuracy is improved from $59 \%$ for baseline BOW model to $71 \%$ for the developed CNN network.

- VIS-NIR 90m, the stratification accuracy improves from 58\% from baseline BOW model to $64 \%$.

- Stratification results are similar for VIS-NIR60m and VIS-NIR 120m and for intra-spectral.

\subsection{Face Matching based on Demographic Information}

The use of demographic filtering (ethnicity and gender class) is developed to improve the performance of face recognition systems. In this work, three different face recognition experiments are conducted: AFIES, COTS and developed MFSDF method. Table 6.5 provides an overview of the number of datasets used, as well as the cross-scenarios and face recognition algorithms selected. In this work, the datasets, with and without the usage of demographic filtering or soft biometric traits are selected. Without grouping, all data is used to perform the face matching experiments. With grouping, the ethnicity class (Caucasian and Asian) and gender class (Male and Female) is used. The stratified database with labels; Male, Female, Asian and Caucasian from the developed deep learning system are used for the data with grouping. For each dataset, the $50 \%$ data is randomly selected as the training set and rest of the data is used as the testing set with no subject overlap. 
Table 6.5: Summary of total number of FR experiments performed, with and without grouping of data in terms of ethnicity and gender class. To perform the FR experiments, two FR crossscenarios are selected cross-distance (CD) and cross-spectral (CS).

\begin{tabular}{|c|c|c|c|c|}
\hline Datasets & $\begin{array}{c}\text { Train/Test } \\
\text { Datasets }\end{array}$ & \# Scenarios & CSU, COTS & $\begin{array}{c}\text { \# Total No. of } \\
\text { Experiments }\end{array}$ \\
\hline All & 4 & $3 \mathrm{CD}+4 \mathrm{CS}$ & $6+1+2$ & 252 \\
\hline Caucasian & 4 & $3 \mathrm{CD}+4 \mathrm{CS}$ & $6+1+2$ & 252 \\
\hline Asian & 4 & $3 \mathrm{CD}+4 \mathrm{CS}$ & $6+1+2$ & 252 \\
\hline Male & 4 & $3 \mathrm{CD}+4 \mathrm{CS}$ & $6+1+2$ & 252 \\
\hline Female & 4 & $3 \mathrm{CD}+4 \mathrm{CS}$ & $6+1+2$ & 252 \\
\hline
\end{tabular}

This process is repeated several times, using random selection of the training and test sets each time. Two cross-scenarios are selected: cross-distance (CD) and cross-spectral (CS).

- For CD, NIR vs. NIR face matching is performed for 3 sets: $30 \mathrm{~m} v s .60 \mathrm{~m}$, $30 \mathrm{~m}$ vs. $90 \mathrm{~m}$ and $30 \mathrm{~m}$ vs. $120 \mathrm{~m}$.

- For CS, VIS vs. NIR face matching is performed for 4 sets: $1.5 m$ vs. $30 m, 1.5 m$ vs. $60 \mathrm{~m}$, $1.5 m$ vs. $90 m$ and $1.5 m$ vs. $120 m$.

- Cross-scenarios are investigated using academic and commercial (non-training based) face matching schemes.

- Each experiment is repeated 4 times for two FR cross-scenarios.

- In total, 252 FR experiment are conducted considering two FR cross-scenarios.

\subsubsection{Demographic-based Cross-Distance Matching}

The face matching experiments are performed for the demographic grouping in terms of gender and ethnicity class.

\section{Gender Class-Male: Cross-Distance Scenarios}

- For $60 m$ and $90 m$ (probe images), it is determined that identifications results are similar for the developed and AFIES method. From developed method, the rank-1 identification accuracy is $94 \%$ for $60 \mathrm{~m}$ and $81 \%$ for $90 \mathrm{~m}$ as shown in Table 6.6 . 
- For $120 m$ distance, developed method performs better and the rank-1 identification rate is improved from $52 \%$ to $71 \%$.

Table 6.6: The intra-spectral, cross-distance matching scenarios for Male Class are investigated. The experimental results when running all FR algorithms using $50 \%$ of the NIR data for training and rest of the data for testing (No overlap of subjects) are presented. For each algorithm including, AFIES, COTS and developed MFSDF, the experiments were run 4 times and the rank-1 average identification accuracy is presented.

\begin{tabular}{|c|c|c|c|}
\hline $\begin{array}{c}\text { FR Algorithm } \\
\text { Rank-1 Identification } \\
\text { Accuracy }\end{array}$ & $\begin{array}{c}\text { Intra-Spectr } \\
30 m \text { vs. } 60 m \\
50 \%\end{array}$ & $\begin{array}{c}\text { I (NIR vs. NII } \\
30 m \text { vs. } 90 m \\
50 \%\end{array}$ & $\begin{array}{c}\text { s-Distance Matching } \\
30 m \text { vs. } 120 m \\
50 \%\end{array}$ \\
\hline MALE DATA & & & \\
\hline $\begin{array}{c}\text { AFIES } \\
\text { Bayesian MAP }\end{array}$ & 0.92 & 0.69 & 0.40 \\
\hline Bayesian ML & 0.93 & 0.74 & 0.47 \\
\hline LDA Euclidean & 0.93 & 0.69 & 0.45 \\
\hline LDA lda_Soft & 0.93 & 0.69 & 0.47 \\
\hline PCA Euclidean & 0.79 & 0.39 & 0.28 \\
\hline PCA MahaCosine & 0.94 & 0.79 & 0.52 \\
\hline COTS & 0.19 & 0.02 & 0.03 \\
\hline Developed Methods & & & \\
\hline MFSDF Without Mask & 0.92 & 0.78 & 0.57 \\
\hline MFSDF With Mask & 0.92 & 0.71 & 0.52 \\
\hline $\begin{array}{l}\text { Fusion of MFSDF (best set) } \\
\text { With and Without Mask }\end{array}$ & 0.94 & $\mathbf{0 . 8 1}$ & 0.71 \\
\hline FEMALE DATA & & & \\
\hline $\begin{array}{c}\text { AFIES } \\
\text { Bayesian MAP }\end{array}$ & 0.78 & 0.53 & 0.38 \\
\hline Bayesian ML & 0.82 & 0.56 & 0.42 \\
\hline LDA Euclidean & 0.74 & 0.47 & 0.38 \\
\hline LDA lda_Soft & 0.75 & 0.46 & 0.39 \\
\hline PCA Euclidean & 0.56 & 0.21 & 0.17 \\
\hline PCA MahaCosine & 0.84 & 0.61 & 0.49 \\
\hline Developed Methods & & & \\
\hline COTS & 0.21 & 0.07 & 0.06 \\
\hline MFSDF Without Mask & 0.65 & 0.51 & 0.44 \\
\hline MFSDF With Mask & 0.71 & 0.47 & 0.41 \\
\hline $\begin{array}{l}\text { Fusion of MFSDF (best set) } \\
\text { With and Without Mask }\end{array}$ & 0.82 & 0.74 & 0.54 \\
\hline
\end{tabular}

Gender Class- Female: Cross-Distance Scenarios For intra-spectral matching (NIR vs. NIR) based on the results:

- For $60 \mathrm{~m}$ (probe images), it is determined that identifications results are similar for developed method and AFIES. From developed method, the rank-1 identification accuracy is 


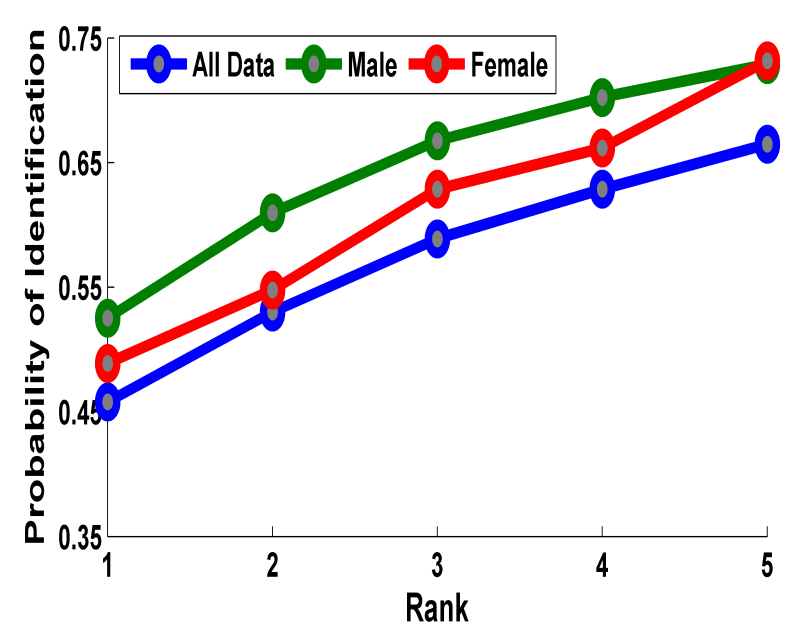

(a) Gender Class vs. All Data

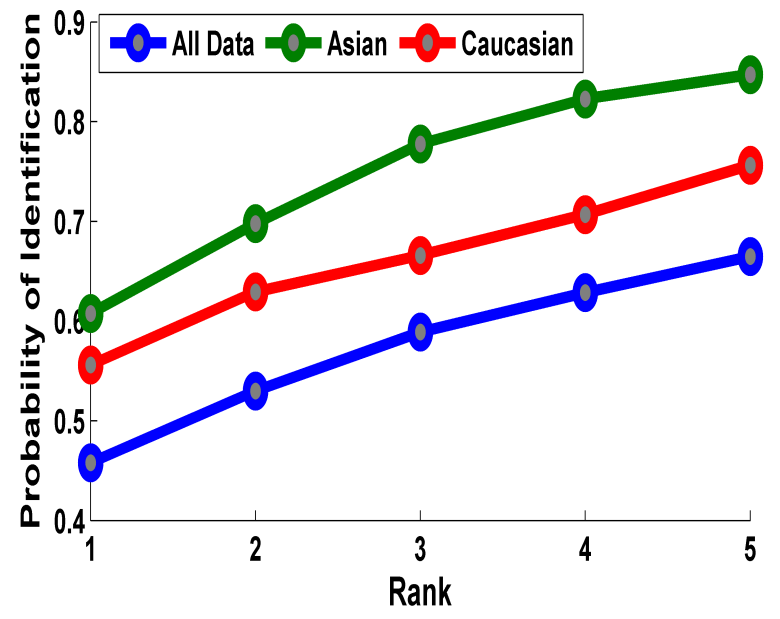

(b) Ethnicity Class vs. All Data

Figure 6.4: Cross-distance matching scenarios for NIR 30m (Gallery) against NIR 120m (Probe): Performance results when the academic CSU face recognition evaluation tool is used. Each algorithm was run several times and the average rank-1 identification accuracy is presented, i.e. (a) Gender class with Male and Female Group vs. all data without any grouping. (b) Ethnicity class with Asian and Caucasian Group against all data without any grouping (Right).

$82 \%$ for $60 m$ as represented in Table 6.6

- For $90 \mathrm{~m}$ and $120 \mathrm{~m}$, it is determined that developed method performs better and the rank1 identification accuracy improved from $61 \%$ to $74 \%$ for $90 \mathrm{~m}$ and $49 \%$ to $54 \%$ for $120 \mathrm{~m}$.

- For the best performed algorithm out of set of algorithms included in the CSU academic matcher, CMC curves for the first 5 ranks are represented in Fig. 6.4 .

- Ethnicity Class: From grouping of the data in terms of ethnicity, the identification performance is improved from $46 \%$ to $61 \%$ for the Asian and $56 \%$ for the Caucasian groups for $120 \mathrm{~m}$ distance data as presented in Table 6.7. The results are similar for $60 \mathrm{~m}$ and $90 \mathrm{~m}$ data, with and without the usage of soft biometric traits in terms of ethnicity. For the first 5 ranks (rank identification accuracy) the CMC curves are represented in Fig. 6.4 for the gender and ethnicity class, for the best performed algorithm out of set of algorithms included in the CSU academic matcher. 
Table 6.7: The intra-spectral, cross-distance matching scenarios for with and without grouping of data in terms of ethnicity class are compared in this table. The experimental results when running all CSU FR algorithms and using 50\% of the NIR data for training and the rest of the data for testing (No overlap of subjects) are presented. The experiments were performed 4 times and the rank-1 average identification accuracy is presented here.

\begin{tabular}{|c|l|l|l|l|l|l|l|l|l|}
\hline $\begin{array}{c}\text { FR Algorithm } \\
\text { Rank-1 Identification } \\
\text { Accuracy }\end{array}$ & \multicolumn{3}{|c|}{$\begin{array}{c}\text { 30 vs. 60 m } \\
\text { 50\% }\end{array}$} & \multicolumn{3}{|c|}{$\begin{array}{c}\text { 30 vs. 90 m } \\
\mathbf{5 0 \%} \%\end{array}$} & \multicolumn{3}{c|}{$\begin{array}{c}\text { 30 vs. 120 m } \\
\text { A0\% }\end{array}$} \\
\hline & All Data & Asian & Caucasian & All Data & Asian & Caucasian & All Data & Asian & Caucasian \\
\hline Bayesian MAP & 0.85 & 0.87 & 0.86 & 0.52 & 0.62 & 0.44 & 0.36 & 0.44 & 0.29 \\
\hline Bayesian ML & 0.86 & 0.85 & 0.88 & 0.60 & $\mathbf{0 . 7 0}$ & 0.60 & 0.38 & 0.50 & 0.38 \\
\hline LDA Euclidean & $\mathbf{0 . 8 8}$ & 0.80 & $\mathbf{0 . 9 0}$ & 0.61 & 0.56 & 0.58 & 0.38 & 0.44 & 0.44 \\
\hline LDA lda_Soft & $\mathbf{0 . 8 8}$ & 0.83 & 0.89 & 0.64 & 0.53 & 0.57 & 0.40 & 0.45 & 0.43 \\
\hline PCA Euclidean & 0.68 & 0.67 & 0.65 & 0.27 & 0.36 & 0.22 & 0.20 & 0.22 & 0.19 \\
\hline PCA MahaCosine & 0.88 & 0.87 & 0.89 & $\mathbf{0 . 6 8}$ & 0.69 & 0.68 & $\mathbf{0 . 4 6}$ & $\mathbf{0 . 6 1}$ & $\mathbf{0 . 5 6}$ \\
\hline
\end{tabular}

\subsubsection{Demographic-based Cross-Spectral Matching}

Cross-spectral face matching experiments are performed in terms of gender and ethnicity class.

Gender Class - Male: For cross-spectral scenario, based on the results for male class, it is determined that developed system outperformed both the commercial and academic software for all four cases, when gallery images (VIS $1 \mathrm{~m}$ ) matched against probe images (NIR 30m, NIR 60m, NIR 90m and NIR 120m) as represented in Table 6.8. The face matching results for:

- 30m, identification performance is improved from 34\% (AFIES), 52\% (COTS) to $61 \%$ using developed approach.

- 60m, identification performance is improved from 33\% (AFIES), 3\% (COTS) to $46 \%$ using developed.

- 90m, identification performance is improved from 2\% for (COTS) to $29 \%$ for Developed and results are similar for AFIES (26\%).

- $\mathbf{1 2 0 m}$, is improved from $18 \%$ (AFIES) to $30 \%$ using developed approach.

Based on the results for female class, for NIR 30m, COTS performs better than both academic and developed system. The demographic filtering and good quality of the images are the main reason for better performance of commercial system. The performance results for (AFIES) and developed method are similar. Moreover, for each of these three FR matchers 


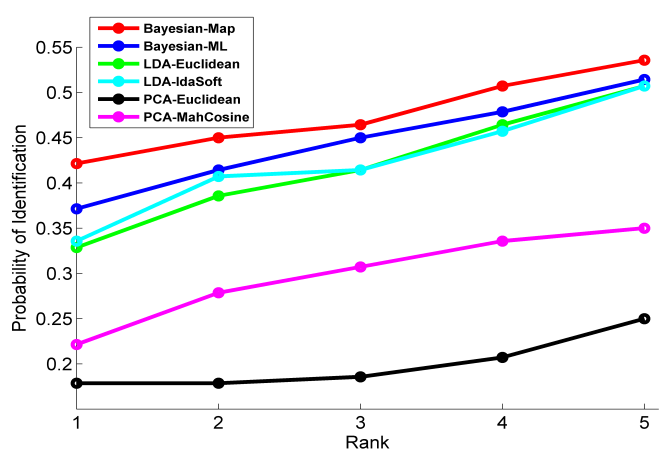

(a) AFIES Face Recognition System

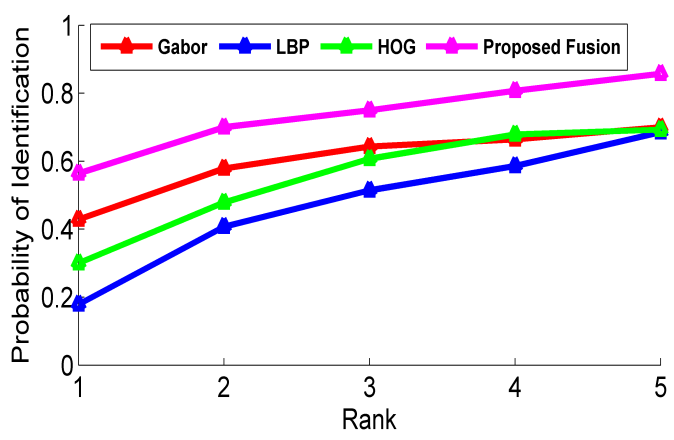

(c) Developed With Mask

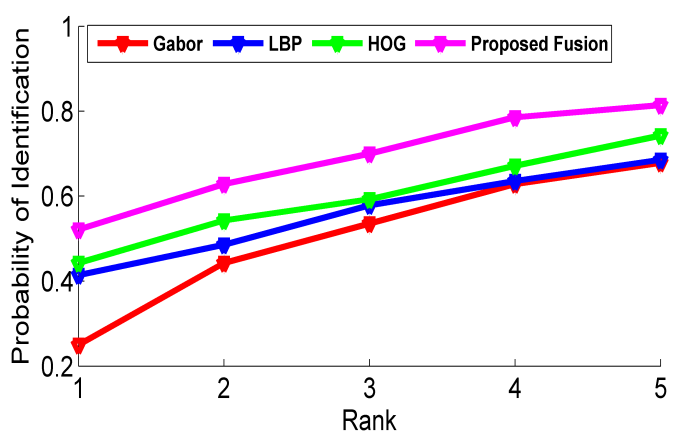

(e) Developed Without Mask

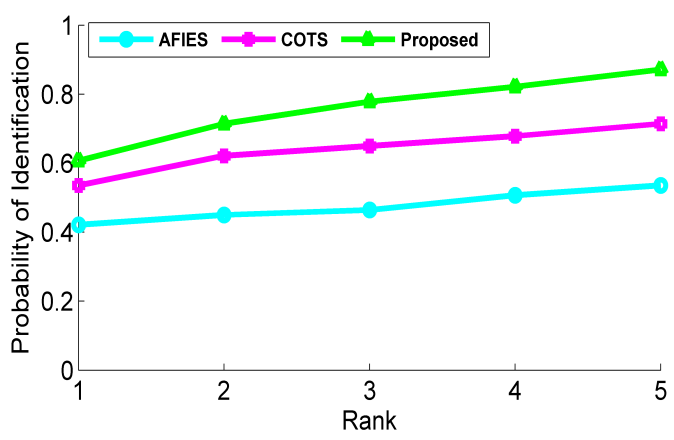

(g) AFIES and COTS vs. Developed

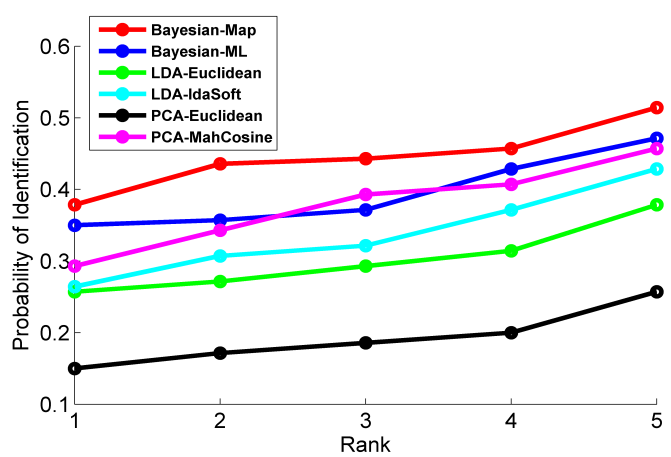

(b) AFIES Face Recognition System

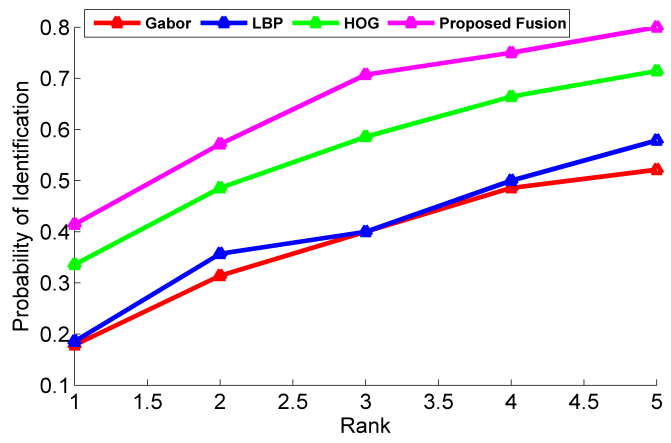

(d) Developed With Mask

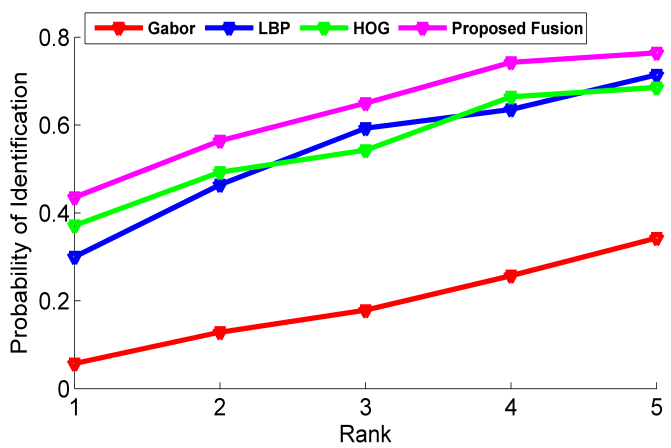

(f) Developed Without Mask

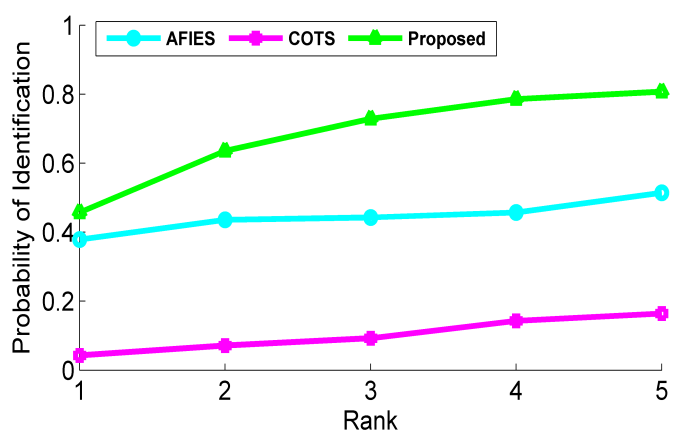

(h) AFIES and COTS vs. Developed

Figure 6.5: Cross-spectral matching scenarios for Male Data, VIS Im (Gallery) against NIR $30 \mathrm{~m}$ (Probe) (Left) and VIS 1m (Gallery) against NIR $60 \mathrm{~m}$ (Probe)(Right). 
Table 6.8: The cross-spectral, cross-distance matching scenarios are investigated. The experimental results when using $50 \%$ of the data for training and the rest data for testing are presented. Experiments were performed 4 times and the rank-1 average identification accuracy is presented.

\begin{tabular}{|c|c|c|c|c|}
\hline $\begin{array}{c}\text { FR Algorithm } \\
\text { VIS 1m vs. }\end{array}$ & \multicolumn{4}{|c|}{ Cross-Spectral (VIS vs. NIR), Cross-Distance Matching } \\
NIR 30 m m & NIR 60 m & NIR 90 m & NIR 120 m \\
(Rank-1 Identification Accuracy) & $\mathbf{5 0 \%}$ & $\mathbf{5 0 \%}$ & $\mathbf{5 0 \%}$ & $\mathbf{5 0 \%}$ \\
\hline MALE DATA & & & & \\
\hline $\begin{array}{c}\text { AFIES } \\
\text { Bayesian MAP }\end{array}$ & 0.34 & 0.33 & 0.18 & 0.14 \\
\hline Bayesian ML & 0.31 & 0.29 & 0.19 & 0.14 \\
\hline LDA Euclidean & 0.30 & 0.27 & 0.25 & 0.17 \\
\hline LDA lda_Soft & 0.32 & 0.28 & 0.26 & 0.18 \\
\hline PCA Euclidean & 0.17 & 0.15 & 0.15 & 0.08 \\
\hline PCA MahaCosine & 0.20 & 0.26 & 0.18 & 0.14 \\
\hline COTS & 0.52 & 0.03 & 0.02 & 0.02 \\
\hline Developed Methods & & & & \\
\hline MFSDF Without Mask & 0.47 & 0.39 & 0.24 & 0.23 \\
\hline MFSDF With Mask & 0.51 & 0.39 & 0.23 & 0.25 \\
\hline Fusion of MFSDF (best set) & & & & \\
\hline With and Without Mask & 0.61 & 0.46 & 0.29 & 0.30 \\
\hline FEMALE DATA & & & & \\
\hline AFIES & & & & \\
\hline Bayesian MAP & 0.30 & 0.25 & 0.17 & 0.12 \\
\hline Bayesian ML & 0.31 & 0.22 & 0.17 & 0.12 \\
\hline LDA Euclidean & 0.26 & 0.21 & 0.18 & 0.14 \\
\hline LDA lda_Soft & 0.27 & 0.23 & 0.18 & 0.12 \\
\hline PCA Euclidean & 0.20 & 0.13 & 0.11 & 0.11 \\
\hline PCA MahaCosine & 0.19 & 0.16 & 0.12 & 0.23 \\
\hline COTS & 0.65 & 0.08 & 0.08 & 0.03 \\
\hline Developed Methods & & & & \\
\hline MFDF Without Mask & 0.26 & 0.22 & 0.18 & 0.19 \\
\hline MFSDF With Mask & 0.32 & 0.27 & 0.26 & 0.16 \\
\hline
\end{tabular}

(AFIES, COTS and developed MFSDF), performance of the system is not improved and the rank-1 identification rate is close to results without any demographic filtering. In some of the cross scenarios, FR system did not perform well and the rank-1 identification accuracy decreased from $23 \%$ (without demographic filtering) to $16 \%$ (AFIES) for NIR 60m, $56 \%$ (without demographic filtering) to $32 \%$ (developed) for NIR $30 \mathrm{~m}$ and $37 \%$ to $27 \%$ (developed) for NIR $30 m$ of the system (Table 6.8).

Based on the results, we can conclude that (for all three FR matchers), female class is more challenging to match in comparison to both male class (with demographic filtering) and all 
data (without filtering). Klare et al.[101] reported that, factors such as, use of cosmetics and small size of female face, results in higher within-class variance and affects the identification performance of system.

- Ethnicity Class: The visible $(1.5 \mathrm{~m})$ to NIR $(30 \mathrm{~m}, 60 \mathrm{~m}, . ., 120 \mathrm{~m})$ face images are matched. In Table 6.9, the results for with and without the usage of soft biometric traits in terms of ethnicity, using both the academic and commercial matchers are shown. In order to examine the effectiveness of these matchers, each experiment is repeated four times, where training set is randomly selected and rest of the data is used for testing. In Table 6.9, the rank-1 average identification accuracy is represented for probe images captured for $30 \mathrm{~m}$ to $120 \mathrm{~m}$ distance.

- For 30m distance, the rank-1 identification accuracy improved from $48 \%$ to $70 \%$ for the Asian group. The results are similar for the Caucasian group when using the COTS matcher.

- For the $60 m$ data, the identification performance improved from $3 \%$ to $48 \%$ for the Asian group.

- For the $90 m$ data, the identification performance improved from $13 \%$ to $32 \%$ for the Asian group while similar results are achieved for the Caucasian group, when academic face matcher is used.

- For the $120 m$ data, the identification accuracy improved from $15 \%$ to $28 \%$ for the Asian group when academic matcher is used.

For all distances, better performance results are achieved for the Asian group in comparison to the original FR system, namely when no gender or ethnicity demographic filtering was used.

The CMC curves (identification accuracy) for the first 5 ranks for the gender and ethnicity classes of algorithms included in the CSU academic matcher, are represented in Fig. 6.6. A set of experiments were performed where each time a different training set was randomly selected.

Commercial matcher is used on all cross-scenarios. The good results are obtained only for the cross-spectral scenario (i.e. visible $1.5 \mathrm{~m}$ gallery against the NIR $30 \mathrm{~m}$ probe images), where 


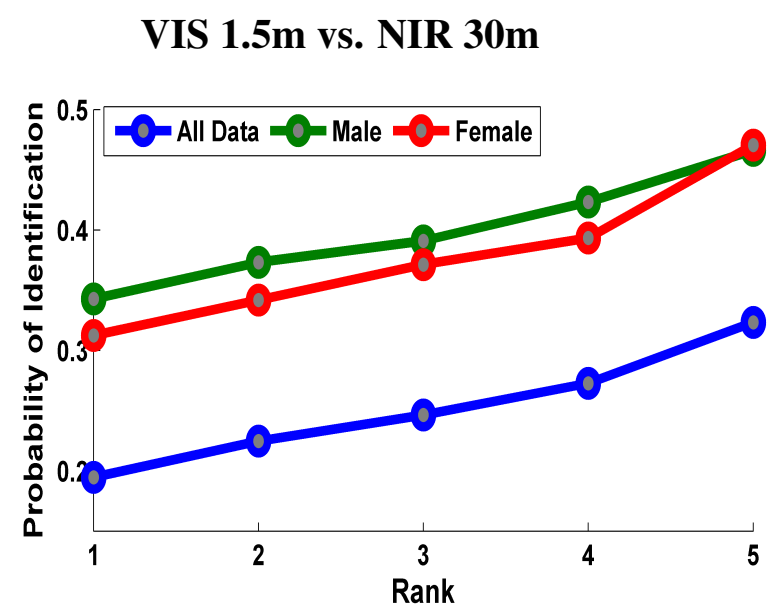

(a) Gender Class vs. All Data

\section{VIS 1.5m vs. NIR 30m}

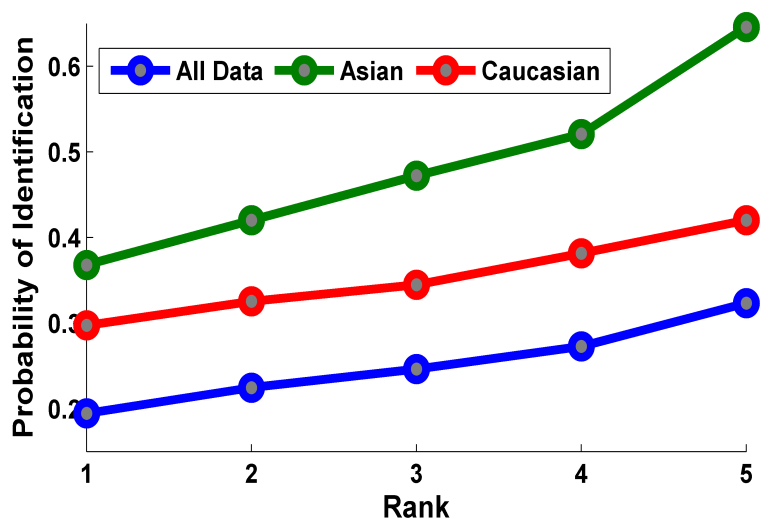

(b) Ethnicity Class vs. All Data

Figure 6.6: Cross-Spectral matching scenarios for VIS $1.5 \mathrm{~m}$ (Gallery) against NIR 30m (Probe): Performance of academic CSU face recognition system. Each algorithm was run several times and the rank-1 identification accuracy is presented. Gender class with Male and Female Group vs. all data without any grouping (Left). Ethnicity class with Asian and Caucasian Group against all data without any grouping (Right).

Table 6.9: The cross-spectral, cross-distance matching scenarios for with and without grouping of data in terms of ethnicity class are compared. The experimental results when running all CSU FR algorithms using 50\% of the NIR data for training and the rest of the data for testing are presented. The experiments were performed 4 times and the rank-1 average identification accuracy is presented. COTS was also tested here.

\begin{tabular}{|c|c|c|c|c|c|c|c|c|c|c|c|c|}
\hline \multirow{3}{*}{$\begin{array}{c}\text { FR Algorithm } \\
\text { Gallery VIS } 1.5 \mathrm{~m} \text { vs. }\end{array}$} & \multicolumn{12}{|c|}{ Cross-Spectral rank-1 identification accuracy for Ethnicity } \\
\hline & \multicolumn{3}{|c|}{ NIR 30m } & \multicolumn{3}{|c|}{ NIR 60m } & \multicolumn{3}{|c|}{ NIR 90m } & \multicolumn{3}{|c|}{ NIR 120m } \\
\hline & All Data & Asian & Caucasian & All Data & Asian & Caucasian & All Data & Asian & Caucasian & All Data & Asian & Caucasian \\
\hline Bayesian MAP & 0.19 & 0.34 & 0.29 & 0.15 & 0.31 & 0.19 & 0.13 & 0.23 & 0.09 & 0.15 & 0.26 & 0.14 \\
\hline Bayesian ML & 0.18 & 0.37 & 0.30 & 0.16 & 0.31 & 0.23 & 0.12 & 0.20 & 0.14 & 0.13 & 0.24 & 0.12 \\
\hline LDA Euclidean & 0.19 & 0.36 & 0.26 & 0.20 & 0.39 & 0.20 & $\mathbf{0 . 1 3}$ & 0.30 & 0.11 & 0.11 & 0.25 & \begin{tabular}{|l}
0.16 \\
\end{tabular} \\
\hline LDA lda_Soft & 0.19 & 0.33 & 0.26 & 0.22 & 0.38 & 0.20 & 0.13 & 0.32 & 0.10 & 0.12 & 0.28 & 0.16 \\
\hline PCA Euclidean & 0.08 & 0.12 & 0.15 & 0.08 & 0.13 & 0.11 & 0.06 & 0.09 & 0.10 & 0.08 & 0.10 & 0.09 \\
\hline PCA MahaCosine & 0.12 & 0.24 & 0.23 & 0.10 & 0.27 & 0.19 & 0.09 & 0.27 & 0.14 & 0.08 & 0.23 & 0.13 \\
\hline COTS & 0.48 & 0.70 & 0.47 & 0.03 & 0.48 & 0.67 & 0.02 & 0.04 & 0.06 & 0.01 & 0.05 & 0.05 \\
\hline
\end{tabular}

images are of good quality. The CMC curves for the first 5 ranks, for the gender and ethnicity class, are shown in Fig. 6.7

\subsubsection{Face Matching With Extended Gallery Data}

In this experiment, to extend the gallery data, Long Distance Heterogeneous Face Database (LDHF) database collected from Kang et al. [71], with 100 subjects is used. LDHF data contains VIS and NIR images at distance of $1 \mathrm{~m}, 60 \mathrm{~m}, 100 \mathrm{~m}$ and $150 \mathrm{~m}$. To perform crossdistance face matching experiments, NIR images collected at distance from $1 \mathrm{~m}$ to $150 \mathrm{~m}$ are selected. For cross-spectral, VIS images at a distance of $1 \mathrm{~m}$ and NIR images at a distance of 


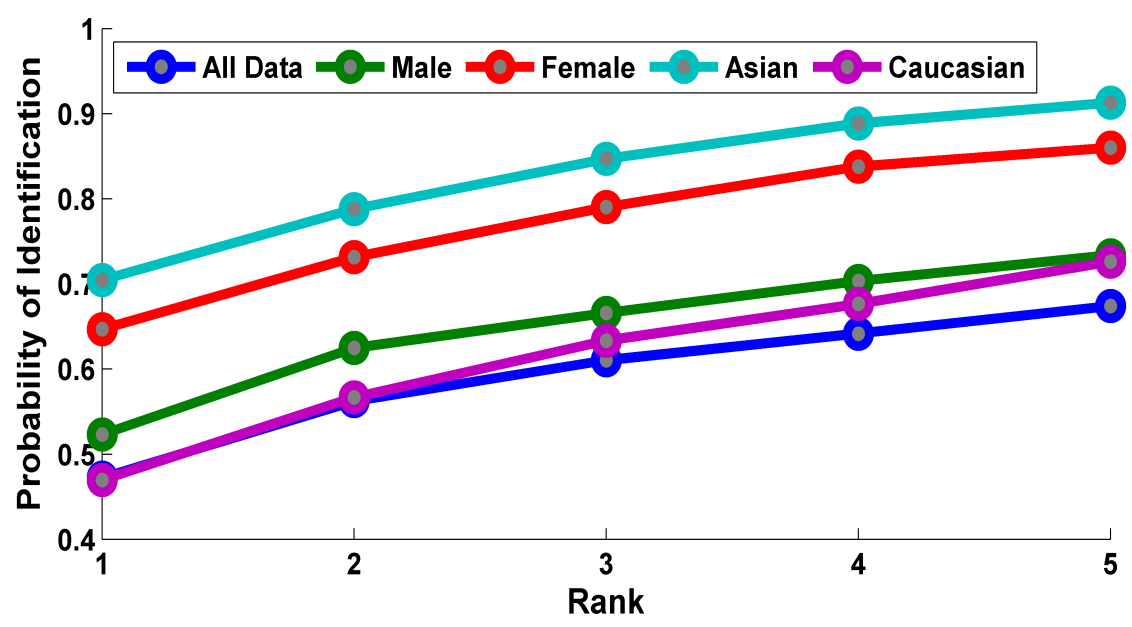

Figure 6.7: Cross-Spectral matching scenarios for VIS (Gallery) against NIR 30m (Probe): Performance of COTS.

$1 \mathrm{~m}, 60 \mathrm{~m}, 100 \mathrm{~m}$ and $150 \mathrm{~m}$ distance are selected. The same set of experiments are performed for with and without demographic filtering using cross-distance and cross-spectral face matching scenarios.

For cross-distance face matching, results are presented in Table 6.10. Based on the results, it is concluded that for $60 \mathrm{~m}$, identification rank-1 performance is higher for AFIES system compared to developed system with and without demographic filtering. For $90 \mathrm{~m}$ and $120 \mathrm{~m}$, developed system achieves better performance results compared to AFIES. For example, the rank-1 identification accuracies at $90 \mathrm{~m}$ and $120 \mathrm{~m}$ distances are $42 \%$ and $30 \%$ (All Data), $48 \%$ and $36 \%$ (Male Class) and 25\% and 20\% (Female Class) respectively.

For cross-spectral scenario, face matching experiments are conducted using 5 different sets. Set 3 to Set 5 are based on selection of both the databases to train the system (our and LDHF data) and rest of the data for testing.

- Set1, where LDHF data base is used for training and our database is used for testing.

- Set2, where our database is used for training and LDHF database is used for testing.

- Set3, LDHF data is used for testing.

- Set4, our data is used for testing.

- Set5, both are used for testing (non-overlapping of training/testing). 
Table 6.10: In this table investigate the intra-spectral, cross-distance matching scenarios with and without demographic filtering, with extended gallery: experimental results when running all FR algorithms using LDHF data for training and our data for testing.

\begin{tabular}{|c|c|c|c|}
\hline \multirow{2}{*}{$\begin{array}{c}\text { FR Algorithm } \\
\text { Rank-1 Identification } \\
\text { Accuracy }\end{array}$} & \multicolumn{3}{|c|}{ Intra-Spectral (NIR vs. NIR), Cross-Distance Matching } \\
\hline & $\begin{array}{c}30 m \text { vs. } 60 m \\
50 \%\end{array}$ & $\begin{array}{c}30 m \text { vs. } 90 m \\
50 \%\end{array}$ & $\begin{array}{c}30 m \text { vs. } 120 m \\
50 \%\end{array}$ \\
\hline \multicolumn{4}{|l|}{ ALL DATA } \\
\hline \multicolumn{4}{|l|}{ AFIES } \\
\hline Bayesian MAP & 0.77 & 0.34 & 0.12 \\
\hline Bayesian ML & 0.78 & 0.38 & 0.20 \\
\hline LDA Euclidean & 0.69 & 0.35 & 0.20 \\
\hline LDA lda_Soft & 0.36 & 0.11 & 0.07 \\
\hline PCA Euclidean & 0.58 & 0.21 & 0.09 \\
\hline PCA MahaCosine & 0.67 & 0.31 & 0.17 \\
\hline \multicolumn{4}{|l|}{ Developed Methods } \\
\hline MFSDF Without Mask & 0.71 & 0.42 & 0.30 \\
\hline \multicolumn{4}{|l|}{ MALE DATA } \\
\hline \multicolumn{4}{|l|}{ AFIES } \\
\hline Bayesian MAP & 0.84 & 0.43 & 0.30 \\
\hline Bayesian ML & 0.84 & 0.42 & 0.30 \\
\hline LDA Euclidean & 0.75 & 0.39 & 0.15 \\
\hline LDA lda_Soft & 0.37 & 0.15 & 0.05 \\
\hline PCA Euclidean & 0.65 & 0.23 & 0.11 \\
\hline PCA MahaCosine & 0.73 & 0.32 & 0.18 \\
\hline \multicolumn{4}{|l|}{ Developed Methods } \\
\hline MFSDF Without Mask & 0.80 & 0.48 & 0.36 \\
\hline \multicolumn{4}{|l|}{ FEMALE DATA } \\
\hline LDA Euclidean & 0.44 & 0.11 & 0.17 \\
\hline LDA lda_Soft & 0.11 & 0.05 & 0.09 \\
\hline PCA Euclidean & 0.41 & 0.13 & 0.15 \\
\hline PCA MahaCosine & 0.46 & 0.13 & 0.07 \\
\hline Developed Methods & & & \\
\hline MFSDF Without Mask & 0.45 & 0.25 & 0.20 \\
\hline
\end{tabular}

Based on the results, it is concluded that the best performance results are achieved from Set3, where both LDHF and our data is used for training and only LDHF database is used for testing. The main reason for better performance for Set 3 is the quality of the images. To verify the face recognition results, a number of image quality assessment (IQA) methods are used, e.g. universal image quality (UIQ), structural similarity index method (SSIM) and feature similarity index for image quality (FSIM). The visible images collected under controlled conditions (VIS $1 \mathrm{~m})$ are used as reference images. The images collected at a distance of $60 \mathrm{~m}$ in NIR band are 
Table 6.11: Measure of statistics (mean, median, minimum and maximum) for attained image quality scores: experimental results when visible images are used as reference images and long distance images (NIR 60m) as query images.

\begin{tabular}{|c|cc|cc|cc|}
\hline IQA Methods & \multicolumn{2}{|c|}{ UIQ } & \multicolumn{2}{|c|}{ SSIM } & \multicolumn{2}{c|}{ FSIM } \\
\hline Data Type & LDHF & OUR & LDHF & OUR & LDHF & OUR \\
Mean & 0.88 & 0.73 & 0.43 & 0.28 & 0.74 & 0.68 \\
Median & 0.90 & 0.74 & 0.43 & 0.28 & 0.75 & 0.68 \\
Minimum & 0.66 & 0.44 & 0.34 & 0.12 & 0.69 & 0.59 \\
Maximum & 0.95 & 0.80 & 0.53 & 0.39 & 0.80 & 0.75 \\
\hline
\end{tabular}

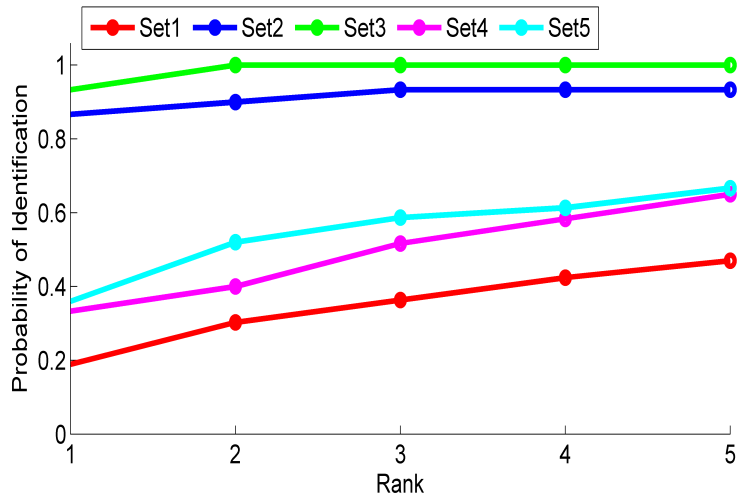

(a) Female: VIS $1 \mathrm{~m}$ vs. NIR 30m

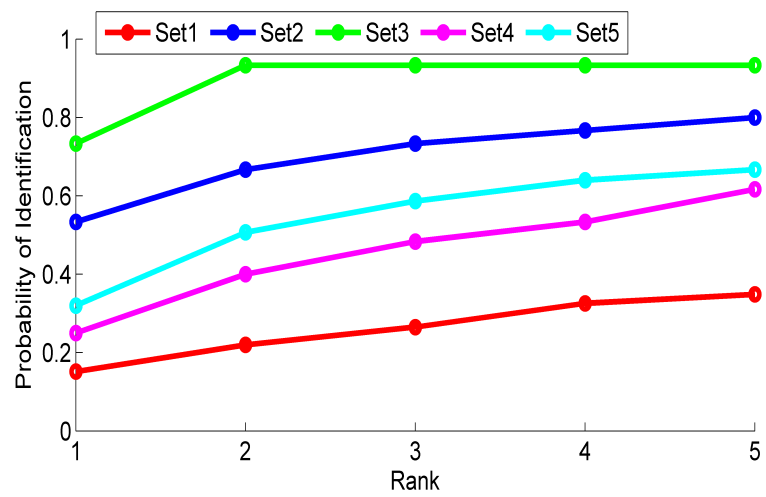

(c) Female: VIS 1m vs. NIR 60m

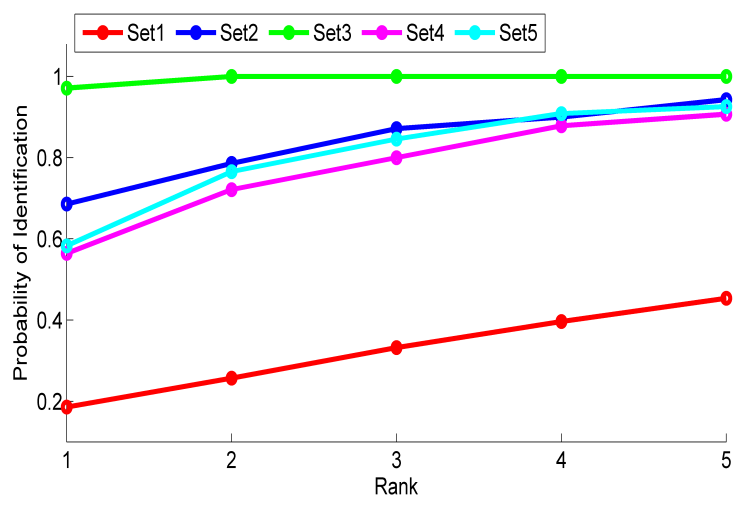

(b) Male: VIS $1 \mathrm{~m}$ vs. NIR 30m

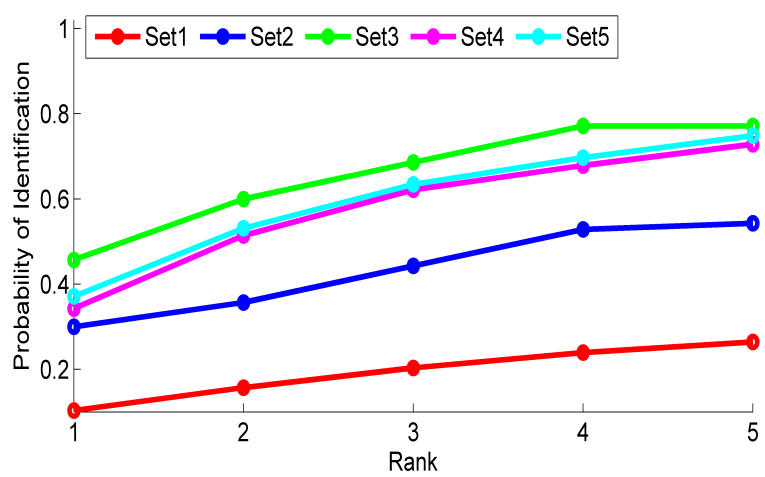

(d) Male: VIS $1 \mathrm{~m}$ vs. NIR $60 \mathrm{~m}$

Figure 6.8: Cross-spectral matching scenarios for VIS 1 m against NIR 30m (Left) and VIS $1 \mathrm{~m}$ against NIR $60 m$ (Right) with extended gallery set (with demographic filtering).

selected as query images. The quality score values lie between 0 and 1 .

Experimental results showed that for LDHF database (100 subjects), quality scores mean value is 0.88 for UIQ, 0.43 for SSIM and 0.74 for FSIM. On the other hand, for our database (103 subjects), quality scores mean value is 0.73 for UIQ, 0.28 for SSIM and 0.68 for FSIM. The statistical analysis results for quality scores (for both the databases) are presented in Tab. 6.11 
It is investigated whether demographic filtering, in particular the gender class (male and female) and extended gallery data, contribute to FR performance under certain conditions, and this has never been investigated in the past, as commented above. For the evaluation of the face identification performance, rank curves $(\mathrm{CMC})$ were generated as shown in Fig. 6.8. In rank curves, the recognition rate is plotted as a function of the rank.

\subsection{Conclusion From Study 3}

\subsubsection{Intra-spectral and Cross-Distance using Developed Approach. Nor- malized images are used to perform the experiments.}

- 30m vs. 60m:, All Data: $93 \%$ - > Male Class $->94 \%->$ Female Class $->82 \%$.

- 30m vs. 90m:, All Data: $68 \%$ - > Male Class $->81 \%->$ Female Class $->74 \%$.

- 30m vs. 120m:, All Data: $64 \%$ - > Male Class $->71 \%->$ Female Class $->54 \%$.

\subsubsection{Cross-spectral and Cross-Distance: Using Developed Approach}

- VIS 1.5 m vs. NIR 30m:, All Data: 56\% - > Male Class - >61\% - > Female Class $->32 \%$.

- VIS 1.5 m vs. NIR 60m: , All Data: 37\% - > Male Class - > 46\% - > Female Class $->27 \%$.

- VIS 1.5 m vs. NIR 90m:, All Data: 23\% - > Male Class - > 29\% - > Female Class $->26 \%$.

- VIS 1.5 m vs. NIR 120m:, All Data: $16 \%$ - > Male Class - > 30\% - > Female Class $->19 \%$.

Based on the conducted study, it is concluded that for both the cross-distance and cross spectral scenarios, developed approach outperformed commercial and academic matchers. 


\subsubsection{Cross-spectral and Cross-Distance for Male Class: COTS vs. Aca- demic vs. Developed Approach}

- VIS $1.5 m$ vs. NIR 30m, the identification improved from 52\% (COTS), 34\% (AFEIS) to $61 \%$ using developed.

- VIS $1.5 \mathrm{~m}$ vs. NIR 60m, the identification improved from 3\% (COTS), 33\% (AFEIS) to $46 \%$ (developed).

- VIS $1.5 m$ vs. NIR 120m, improved from $2 \%$ (COTS), $18 \%$ (AFEIS) to 30\% (developed).

In this experiment cross-distance images, i.e. the baseline NIR images $(30 \mathrm{~m})$ to NIR images captured at $60 \mathrm{~m}, 90 \mathrm{~m}$ and $120 \mathrm{~m}$ respectively are compared. Equal importantly, as a case study (see more information below) it is also investigated that whether demographic filtering can assist in improving cross-distance and cross-spectral FR performance. With extended gallery and with more variation in the training data (including data collected under different conditions such as sensors, distances e.g. LDHF data is used for training and our data for testing and vice versa, both the databases are used for training and testing), it is shown that, it will improve the robustness of the face recognition method as expected.

\subsection{Threats to Validity}

In this chapter, before conducting the face matching experiments, deep learning based networks are developed for the demographic filtering in terms of gender and ethnicity class. The networks are developed to improve the performance of face recognition system.

\subsubsection{Construct Validity}

To construct an efficient classification network, the model hyper parameters were empirically optimized and the models were trained for this challenging database. The visible and NIR databases were selected to conduct the experiments.

Threats from Developed Study: 
- For the database collected under variable light conditions, environment conditions, face poses, face expressions, and camera focus can affect the performance of classification model. These factors act like confounding factor.

- Confounding factor is when the database collected from a specific race or ethnicity class. For the demographic filtering in terms of ethnicity, when the model is trained for a specific class, it results in poor performance for the race or groups those are not included in the model. In this study, the database consists of Caucasian, Asian and Hispanic class. The database was very limited for Hispanic class and in Asian class no middle eastern subjects were available in infrared band images. This is one of the limitation of this study. Hence, to use the network for new ethnic class, researchers need to train the network for the classes those are not present in the database to generate the models. Otherwise, it may result in poor performance.

- Although deep learning networks are robust, the classification performance is affected by selected set of values for hyper parameters (epoch, momentum and learning rate) and the database selected to train the network. These factors are defined as confounding factors and are responsible for variation in the performance. To deal with this threat, the CNN network is optimized, the network is trained from the scratch and the hyper parameters are selected where the highest classification accuracy results are achieved. Finally, this is evaluated on the database collected under challenging conditions (in night time and at long standoff distances). Hence, any change in the values for hyper parameters and training size from the researchers may affect the performance of the system.

\subsubsection{Internal Validity}

In this study, a CNN network is developed based on MatConvNet toolbox. Based on the results, it is claimed that demographic filtering improves the performance of face recognition system.

\section{Threats from Developed Study:}

- Performance of the developed network is dependent on the number of layers (Convolu- 
tion layer, pooling layer, activation layer and fully connected layer). Any change in the network, e.g. change in the layers and the order of layers, change in the filter size for convolution layer, and change in activation function for activation layer may affect the performance of the system.

- Performance of the classification network is dependent on the size of normalized images (using image registration). To use the new datasets, researchers need to resize the face images to size of $32 \times 32$ pixels, otherwise it may result in poor performance.

- Performance of the developed face matching approach is dependent on the training data set (Visible and NIR band). Hence, to use the new datasets (Face database in SWIR and Thermal band), researchers need to retrain the system.

\subsubsection{Conclusion Validity}

The developed classification network for the demographic filtering is reliable and achieved output results are same when the experiments are repeated using random training and testing sets.

\section{Threats from Developed Study:}

- System is developed in Matlab environment using MatConvNet. The developed system is compatible for Matlab R2014b or later, NVIDA GPU card with compute capability of 3.0 or greater and CUDA toolkit version 6.0 or later.

- CNN based classification network is working well for academic database with 103 subjects. In a scenario, where the researcher wants to train the system on new database, they need to consider the sufficient amount of database to train the system, otherwise the performance of the system may be affected from small sample size.

\subsubsection{External Validity}

The validity is concerned about generalization. To make the system more generalized and robust, the classification and face matching experiments are performed with extended database 
(LDHF). Based on the results, it is concluded that designed model improved the performance of system.

\section{Threats from Developed Study:}

- The threat may be caused if the developed system performs well only for a specific database and not for the unseen database. In this study, when LDHF database is selected to perform the classification, better classification results achieved for gender class. However, for the ethnicity class the results were not satisfactory. LDHF database consists of primarily Asian population and to deal with this threat databases over scattered ethnic groups are selected. 


\section{Chapter 7}

\section{Developed Image Restoration Scheme for Night Time Long Range Imagery System in NIR Band}

The focus of this chapter is to determine whether the restored images from the developed image restoration method in study 3 can enhance recognition performance of the un-constrained database collected in NIR band.

\subsection{Baseline Face Matching Results using developed Image Restoration}

Fig. 7.1, shows the extent to which the performance of a FR system is improved by selected set of pre-processing combinations (see Fig. 4.7 for selected sets of combinations). Based on the results, it is concluded that the selection of a certain combination of pre-processing techniques is critical in improving cross-spectral matching performances in terms of rank-1 identification accuracy.

The experimental results demonstrate that when the LBP/LTP descriptors are employed:

- Better results are obtained for comb2, when first use $D N$ and then $P N$ (single scale self quotient). 
- This order (comb 2) results in $40 \%$ rank-1 identification accuracy (e.g. for LBP-CHI)

- 30 percent point (pp) improvement using developed restoration method in study 2 in comparison to when using the raw images without selected pre-processing combination (No Norm).

Similar results are achieved for other descriptors and distance metrics (see Fig. 7.1).

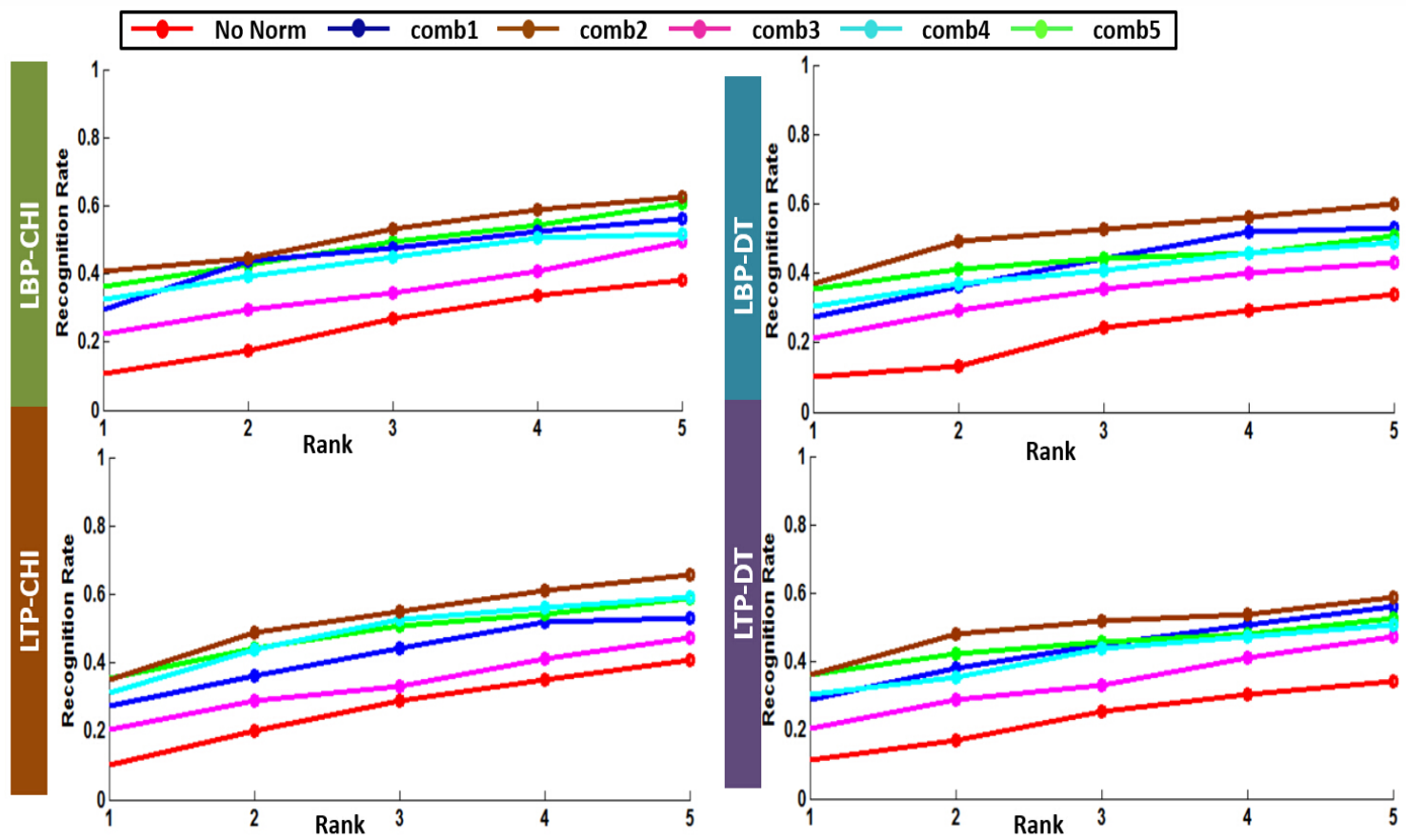

Figure 7.1: CMC curves comparing the identification performance (first 5 ranks) for cross-spectral matching (Gallery-VIS and Probe-NIR): LBP-CHI (top-left), LBP-DT (top-right), LTP-CHI (bottomleft) and LTP-DT (bottom-right). Five pre-processing combinations selected such as comb1, comb2, comb3, comb4 and comb5.

\subsection{Matching using developed Image Restoration and Fusion Scheme}

The experimental results indicate that for LBP/LTP based methods, the best overall identification accuracy reaches $40 \%$ using developed image restoration scheme and hence these results are not satisfying. Thus, an alternative, more complicated, but much more efficient approach is investigated. The developed image restoration and fusion multi-feature dependent fusion schemes are combined (as described in Chapter 5). The DT and CHI distance transform 
methods are replaced by a kernel subspace method [77]. To train the system, 52 subjects and 8 samples are used per subject ( 4 face images in VIS and 4 face images in NIR band for each subject). The rest of the data is used for testing, i.e. 4 face images for each test subject in the visible band for gallery set and 4 face images in the NIR band for probe set. Note that there is no subject overlap between the training and test sets.

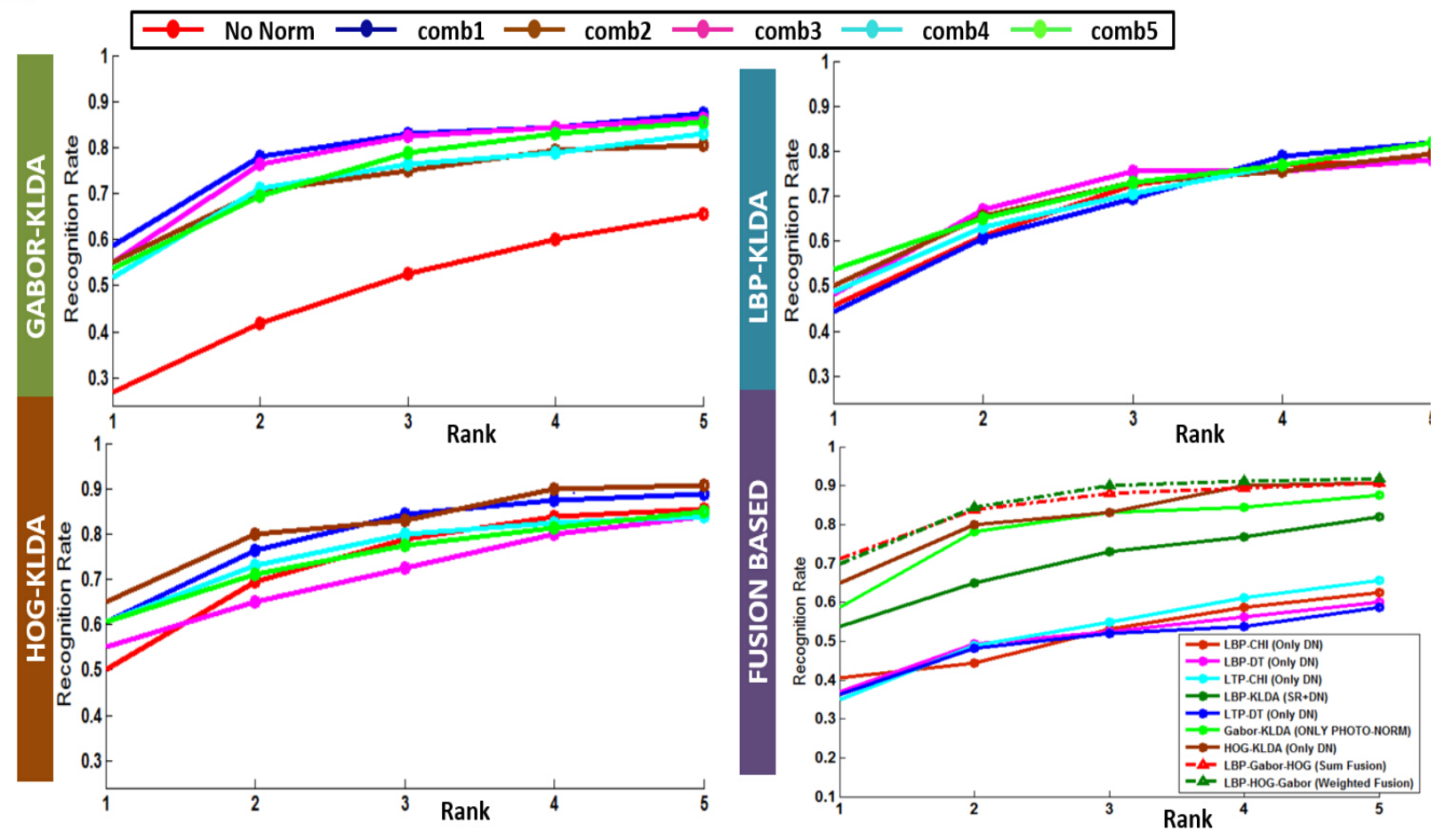

Figure 7.2: CMC curves comparing identification performance (for rank to rank 5) results, for crossspectral matching, for five selected combinations of pre-processing techniques: LBP/KLDA (top-right), GABOR/KLDA (top-left) and HOG/KLDA (bottom-left).

The same procedure is followed as applied before when using the LBP and LTP descriptors including: usage of various pre-processing combinations based on developed image restoration approach following the $P N$ method. 5 different combinations for pre-processing (see Fig. 4.7) are investigated and, finally, the best combination is selected based on the performance results. It is concluded that (as shown in Fig. 7.2), the selection of the combination of pre-processing technique is critical in improving the matching performance for each selected feature descriptor.

First the PN is selected, for each feature descriptor and the best combination that provided us with best rank-1 identification accuracy is identified. The results are shown in Fig.7.2. The experimental results demonstrate that when HOG/KLDA descriptor is employed, better results 
are obtained for comb2, i.e. when first denoising and then isotropic smoothing is used. This ordered pre-processing of the face images results in $65 \%$ rank-1 identification accuracy (see bottom-left table in Fig. 7.2).

When the LBP/KLDA descriptor is used, better results are obtained for comb5, where, first, super-resolution, second, denoising and, then, single scale self quotient normalization are used. This ordered pre-processing of the face images results in 53\% rank-1 identification accuracy (see top-left Fig. 7.2). Finally, when the Gabor/KLDA descriptor is used, better results are obtained for combl, i.e. when the Tann and Triggs photometric normalization technique is used. This order results in 58\% rank-1 identification accuracy (see top-right Fig. 7.2). The selection of pre-processing combination and PN techniques depend on the feature descriptor used. For each pre-processing combination tested, the best achieved rank-1 identification accuracy is presented in the table found at the bottom right corner of Fig. 7.2 .

\section{Face Matching Results for All Data using Study 1 followed by Study 3}

- For HOG descriptor better results are obtained for comb2 and the rank-1 identification accuracy improved from $50 \%$ to $65 \%$.

- For LBP descriptor better results are obtained for combl and the rank-1 identification accuracy improved from $20 \%$ to $53 \%$.

- Based on developed fusion scheme (Study 1), where selected each feature descriptors with the best selected combination for image restoration (study 3), 70\% rank-1 identification accuracy is achieved.

\subsection{Matching using developed Image Restoration, Fusion Scheme and Demographic Information}

The use of demographic information (soft biometrics) before the face matching experiments can improve the performance of traditional FR systems. Soft biometric traits are physical and behavioral features (weight, height, gender, ethnicity, etc.), and offer several advantages over the traditional systems. In this work, three main strata or traits: gender, ethnicity and facial hair 
are considered. For female data, the database is grouped into Female Asian (FA) and Female Caucasian (FC) class. For male data, the database is grouped into male with beard (MWB) and male without beard (MWOB) class.

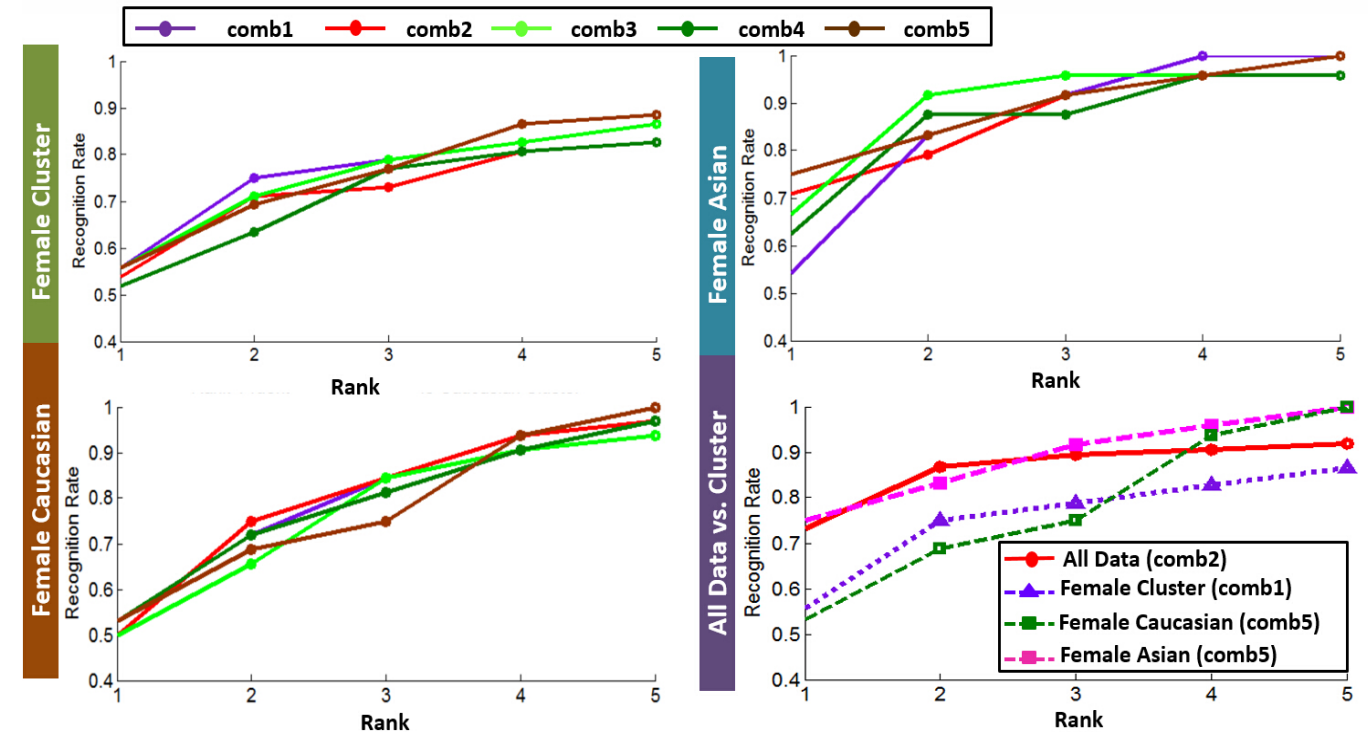

Figure 7.3: Cross-spectral matching results for Female Stratum: CMC curves comparing the performance for the face images restored from the developed method with raw images.

Based on the face identification results for female stratum as shown in Fig. 7.3 , it is concluded that selection of combination of pre-processing technique (comb1, comb 2,..., comb5) is the key factor in improving the matching performance. The experimental results demonstrate that for FA stratum, better results are obtained for comb5, when first $S R$, second $D N$ and then $P N$ (adaptive single scale retinex named as norm 2 see in top-right Fig. 7.3) are used. The performance of the system is improved (rank-1 identification accuracy) from $55 \%$ to $75 \%$. For FC stratum, results are similar for all the combinations (see in bottom-left). The comb5 is selected, based on results for first 5 ranks and this combination results in 53\% rank-1 identification accuracy. For a comparison between with and without the usage of stratification of the database results are presented in bottom-right. The results (rank) from face identification experiments are verified with results from statistical tests.

The same set of experiments are repeated for MWB and MWOB class. Based on the statistical hypothesis results, it is evaluated that comb5 for MWOB and comb2 for MWB are selected as the best combinations. The rank-1 identification accuracies with best results using the five pre-processing combinations for MWB and MWOB are shown in Fig. 7.4. Based on 
the results, it is concluded that better performance results are obtained from comb2 for MWB and from comb5 for MWOB. The performance of the system is improved (rank-1 identification accuracy) from 58\% to $75 \%$ for MWOB (bottom left), for comb5, $63 \%$ to $82 \%$ for MWB (top right).

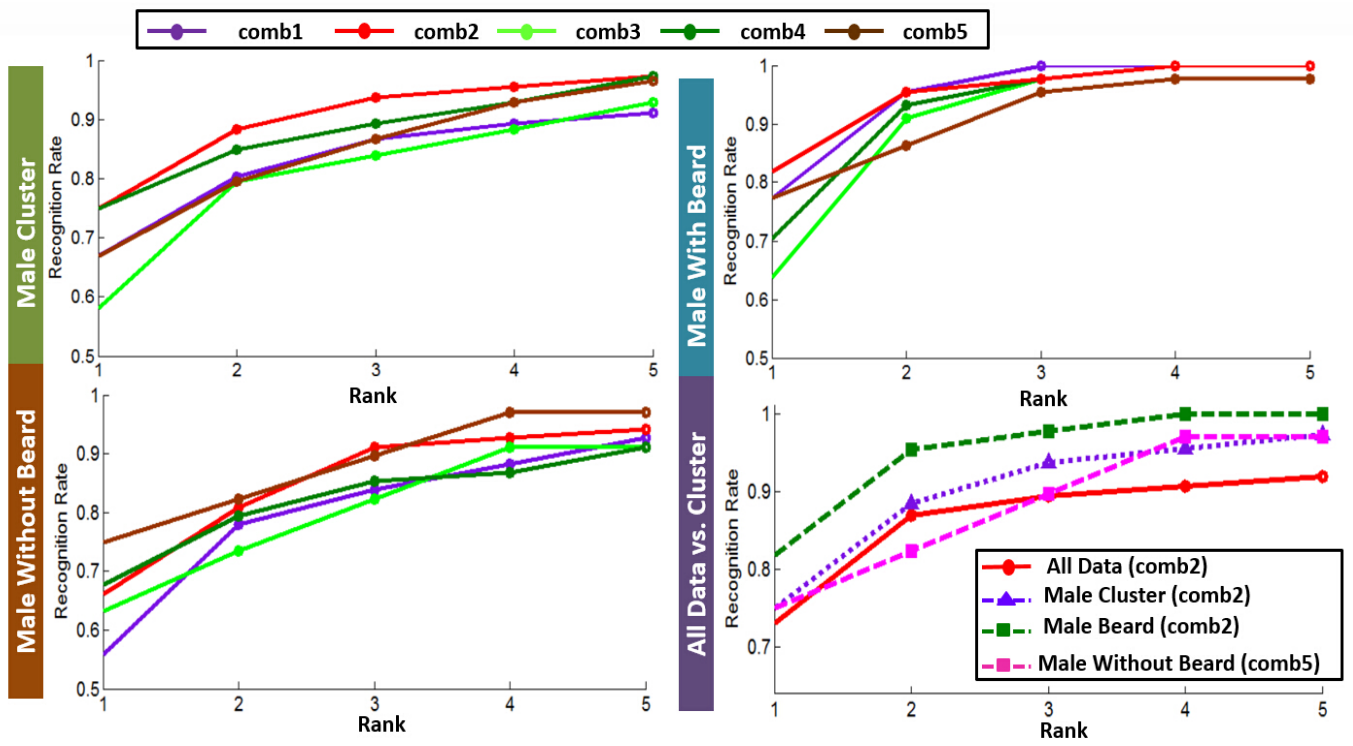

Figure 7.4: Cross-spectral matching results for Male Cluster: CMC curves comparing the performance of FR system for the face images restored from the developed method with raw images.

\subsubsection{Cross-Matching results for Long Distances}

Each experiment (five scenarios: All Database, MWB, MWOB, FA and FC) is repeated 10 times to remove the bias in the results. The training and testing data are randomly selected. For the scenario without the usage of demographic information (All Data), the best rank-1 identification ranks are reported in Table 7.1. For the selected set of soft biometric traits, the best rank-1 identification accuracies are reported in Table 7.2. The same set of experiments are performed (10 times) for NIR database at distance of $60 \mathrm{~m}, 90 \mathrm{~m}$ and $120 \mathrm{~m}$ for the five scenarios (All Database, FA, FC, MWB and MWOB).

Based on the results for the scenario with all the database for 4 different distances NIR $30 m, 60 m, 90 m$ and $120 m$ (see Table 7.1), it was determined that for all distances, better performance results are achieved from the developed methods in comparison to the commercial off the shelf (COTS) G8 system (L1 Systems). 
Table 7.1: Cross-spectral Matching Results (Gallery in VIS (1m-indoor) under controlled conditions and Probe images in NIR): Rank identification accuracies for probe images at 4 different distances in nighttime environment: $30 \mathrm{~m}, 60 \mathrm{~m}, 90 \mathrm{~m}$ and $120 \mathrm{~m}$ distance.

\begin{tabular}{|c|l|l|l|l|l|}
\hline Rank Identification Accuracy & R1 & R2 & R3 & R4 & R5 \\
\hline Probe Images at 30 m & & & & & \\
\hline Pre-processing : Combination & & & & & \\
\hline comb1 & $\mathbf{0 . 7 3}$ & $\mathbf{0 . 8 6}$ & $\mathbf{0 . 8 8}$ & $\mathbf{0 . 9 1}$ & $\mathbf{0 . 9 2}$ \\
comb2 & 0.73 & 0.86 & 0.89 & 0.90 & 0.91 \\
comb3 & 0.69 & 0.83 & 0.85 & 0.91 & 0.94 \\
comb4 & 0.67 & 0.83 & 0.89 & 0.91 & 0.92 \\
comb5 & 0.70 & 0.78 & 0.82 & 0.85 & 0.91 \\
COTS & $\mathbf{0 . 4 8}$ & $\mathbf{0 . 5 1}$ & $\mathbf{0 . 5 5}$ & $\mathbf{0 . 6 0}$ & $\mathbf{0 . 6 2}$ \\
\hline Probe Images at 60m & & & & & \\
\hline comb1 & 0.44 & 0.59 & 0.69 & 0.74 & 0.79 \\
comb2 & $\mathbf{0 . 5 6}$ & $\mathbf{0 . 6 7}$ & $\mathbf{0 . 7 6}$ & $\mathbf{0 . 8 4}$ & $\mathbf{0 . 8 6}$ \\
comb3 & 0.51 & 0.61 & 0.70 & 0.77 & 0.85 \\
comb4 & 0.48 & 0.63 & 0.73 & 0.77 & 0.83 \\
comb5 & 0.39 & 0.54 & 0.61 & 0.68 & 0.78 \\
COTS & $\mathbf{0 . 0 1}$ & $\mathbf{0 . 0 2}$ & $\mathbf{0 . 0 3}$ & $\mathbf{0 . 0 4}$ & $\mathbf{0 . 0 5}$ \\
\hline Probe Images at 90 $\mathbf{m}$ & & & & & \\
\hline comb1 & 0.20 & 0.30 & 0.40 & 0.45 & 0.52 \\
comb2 & 0.25 & 0.33 & 0.40 & 0.47 & 0.53 \\
comb3 & $\mathbf{0 . 3 0}$ & $\mathbf{0 . 3 9}$ & $\mathbf{0 . 4 9}$ & $\mathbf{0 . 5 4}$ & $\mathbf{0 . 5 9}$ \\
comb4 & 0.22 & 0.30 & 0.36 & 0.40 & 0.44 \\
comb5 & 0.24 & 0.30 & 0.37 & 0.41 & 0.47 \\
COTS & $\mathbf{0 . 0 1}$ & $\mathbf{0 . 0 1}$ & $\mathbf{0 . 0 2}$ & $\mathbf{0 . 0 2}$ & $\mathbf{0 . 0 4}$ \\
\hline Probe Images at 120 m & & & & & \\
\hline comb1 & 0.16 & 0.26 & 0.37 & 0.41 & 0.47 \\
comb2 & 0.23 & 0.37 & 0.44 & 0.51 & 0.54 \\
comb3 & $\mathbf{0 . 2 8}$ & $\mathbf{0 . 4 0}$ & $\mathbf{0 . 4 8}$ & $\mathbf{0 . 5 2}$ & $\mathbf{0 . 5 8}$ \\
comb4 & 0.20 & 0.30 & 0.36 & 0.42 & 0.46 \\
comb5 & 0.18 & 0.28 & 0.32 & 0.36 & 0.43 \\
COTS & $\mathbf{0 . 0 1}$ & $\mathbf{0 . 0 1}$ & $\mathbf{0 . 0 2}$ & $\mathbf{0 . 0 4}$ & $\mathbf{0 . 0 4}$ \\
\hline & & & & \\
\hline
\end{tabular}

- For $30 m$ distance, performance reaches almost $48 \%$ for COTS system, whereas, the accuracy reaches almost $73 \%$ when using the developed approach. For the $30 \mathrm{~m}$ distance dataset, the rank-1 identification accuracy is very close to each other for combl and comb2. The comb1 is selected based on a comparison among the first 5 identification ranks.

- For the $60 m$ NIR images collected at long standoff distance, the rank-1 identification accuracy is very low when the COTS system software is used. The better performance results are achieved from the developed pre-processing and fusion schemes, i.e. the rank1 identification accuracy from developed approach reaches to $56 \%$ for comb2 (see Table 
7.1).

- For the $90 \mathrm{~m}$ images collected at long standoff distance, the rank-1 accuracy is $1 \%$ when the COTS system software is used. The better performance results are achieved from the developed pre-processing and fusion schemes, i.e. the rank-1 accuracy reaches to $30 \%$ for comb3.

- Finally, for $120 \mathrm{~m}$ images the rank-1 accuracy from developed approach reaches to $28 \%$ for comb3.

Table 7.2: Cross-spectral matching results for Soft Biometric Traits (Gallery in VIS ( $1 m$-indoor) under controlled conditions and Probe images in NIR): Rank identification accuracy for probe images at 4 different distances in nighttime environment: $30 \mathrm{~m}, 60 \mathrm{~m}, 90 \mathrm{~m}$ and $120 \mathrm{~m}$ distance.

\begin{tabular}{|c|c|c|c|c|c|}
\hline $\begin{array}{c}\text { Rank Identification Accuracy } \\
\text { Distances }\end{array}$ & R1 & $\mathbf{R 2}$ & $\mathbf{R 3}$ & R4 & R5 \\
\hline \multicolumn{6}{|l|}{ Female Asian: } \\
\hline $30 \mathrm{~m}$ & 0.75 & 0.83 & 0.91 & 0.95 & 1.00 \\
\hline $60 \mathrm{~m}$ & 0.37 & 0.58 & 0.79 & 0.96 & 0.96 \\
\hline $90 \mathrm{~m}$ & 0.41 & 0.54 & 0.67 & 0.87 & 1.00 \\
\hline $120 \mathrm{~m}$ & 0.38 & 0.58 & 0.75 & 0.91 & 0.96 \\
\hline \multicolumn{6}{|l|}{ Female Caucasian: } \\
\hline $30 \mathrm{~m}$ & 0.53 & 0.68 & 0.75 & 0.93 & 1.00 \\
\hline $60 \mathrm{~m}$ & 0.50 & 0.66 & 0.72 & 0.84 & 0.84 \\
\hline $90 \mathrm{~m}$ & 0.28 & 0.47 & 0.63 & 0.78 & 0.88 \\
\hline $120 \mathrm{~m}$ & 0.25 & 0.47 & 0.56 & 0.65 & 0.75 \\
\hline \multicolumn{6}{|l|}{ Male With Beard: } \\
\hline $30 \mathrm{~m}$ & 0.82 & 0.95 & 0.97 & 1.00 & 1.00 \\
\hline $60 \mathrm{~m}$ & 0.52 & 0.73 & 0.89 & 0.98 & 0.98 \\
\hline $90 \mathrm{~m}$ & 0.43 & 0.54 & 0.75 & 0.84 & 0.90 \\
\hline $120 \mathrm{~m}$ & 0.38 & 0.52 & 0.68 & 0.80 & 0.82 \\
\hline \multicolumn{6}{|l|}{ Male Without Beard: } \\
\hline $30 \mathrm{~m}$ & 0.75 & 0.82 & 0.89 & 0.97 & 0.97 \\
\hline $60 \mathrm{~m}$ & 0.49 & 0.72 & 0.80 & 0.84 & 0.88 \\
\hline $90 \mathrm{~m}$ & 0.38 & 0.53 & 0.58 & 0.65 & 0.71 \\
\hline $120 \mathrm{~m}$ & 0.27 & 0.43 & 0.50 & 0.61 & 0.65 \\
\hline
\end{tabular}


Table 7.3: In this table investigate the cross-spectral, cross-distance matching scenarios for database before and after the usage of developed image restoration and demographic filtering of male class into with or without beard and female class into Asian and Caucasian class: Experiments were run several times and the rank- 1 identification accuracy presented here (average). Developed-Set 1: Only Developed Fusion Scheme applied to the images, Developed-Set 2: Both developed image restoration and fusion scheme applied to the images. For Set 2 with Male and Female Class further sub-clustering is performed: MWB, MWOB, FA and FC class

\begin{tabular}{|c|c|c|c|c|}
\hline Rank-1 Identification Accuracy & \multicolumn{3}{|c|}{ Cross-Spectral (VIS vs. NIR), Cross-Distance Matching } \\
VIS 1.5m vs. & NIR 30 m & NIR 60 m & NIR 90 m & NIR 120 m \\
\hline ALL DATA & & & & \\
AFIES & 0.19 & 0.22 & 0.21 & 0.15 \\
COTS & 0.48 & 0.03 & 0.02 & 0.01 \\
Developed-Set 1 & 0.56 & 0.37 & 0.23 & 0.16 \\
Developed-Set 2 & $\mathbf{0 . 7 3}$ & $\mathbf{0 . 5 6}$ & $\mathbf{0 . 3 0}$ & $\mathbf{0 . 2 8}$ \\
\hline MALE Class & & & & \\
AFIES & 0.34 & 0.33 & 0.26 & 0.18 \\
COTS & 0.52 & 0.03 & 0.02 & 0.02 \\
Developed-Set 1 & 0.61 & 0.46 & 0.29 & 0.30 \\
Developed-Set 2: MWB & $\mathbf{0 . 8 2}$ & 0.52 & $\mathbf{0 . 4 3}$ & 0.38 \\
Developed-Set 2: MWOB & $\mathbf{0 . 7 5}$ & 0.49 & $\mathbf{0 . 3 8}$ & 0.27 \\
\hline Female Class & & & & \\
AFIES & 0.30 & 0.25 & 0.18 & 0.23 \\
COTS & 0.65 & 0.08 & 0.08 & 0.03 \\
Developed-Set 1 & 0.32 & 0.27 & 0.26 & 0.16 \\
Developed-Set 2: FA & $\mathbf{0 . 7 5}$ & 0.37 & 0.41 & 0.38 \\
Developed-Set 2: FC & 0.53 & 0.50 & 0.28 & 0.25 \\
\hline \multicolumn{2}{|}{}
\end{tabular}

\subsection{Conclusion from Study 3}

\subsubsection{Comparison between Developed Study 1 vs. Academic and Com- mercial Matcher}

- For All Database (without usage of demographic information)

- Rank-1 identification accuracy improved from $48 \%$ for COTS system, $19 \%$ for AFIES to $56 \%$ when using developed approach for (see in Table 7.3) $30 \mathrm{~m}$ distance.

- Rank-1 identification accuracy improved from 3\% for COTS system, 22\% for AFIES to $37 \%$ using developed approach.

- Rank-1 identification accuracy improved from $3 \%$ for COTS system to $23 \%$ using developed approach and results are similar for AFIES for $90 \mathrm{~m}$ distance.

- Rank-1 identification accuracy improved from $1 \%$ for COTS system to $16 \%$ using devel- 
oped approach and results are similar for AFIES for $120 m$ distance.

\subsubsection{Comparison between Developed Study 1 vs. Study 1 (Fusion Scheme) followed by Study 3 (Image Restoration)}

- For All Database (without usage of demographic information)

- Rank-1 identification accuracy improved from $56 \%$ to $73 \%$ for $30 m$ distance (see in Table 7.3 ).

- Rank-1 identification accuracy improved from $37 \%$ to $56 \%$ for $60 \mathrm{~m}$ distance.

- Rank-1 identification accuracy improved from $23 \%$ to $30 \%$ for $90 \mathrm{~m}$ distance.

- Rank-1 identification accuracy improved from $16 \%$ to $28 \%$ for $120 \mathrm{~m}$ distance.

The identification experiments are performed for the database with demographic information or soft biometric traits (FA, FC, MWB and MWOB) and each experiment is repeated 10 times (randomly selected training and test data) and results from best set is reported in Table 7.3 .

\section{- With usage of demographic information: Female Class for 30m distance}

- Female Class, rank-1 identification accuracy improved from 32\% (Only Study 1) (see in Table 7.3) to 58\% where Study 1 followed by Study 2 and Study 3 (see Fig. 7.3).

- Female Asian Class, rank-1 identification accuracy improved from 58\% to 75\% (Study1 followed by Study 2 and Study 3), see in Table 7.3.

- Results are similar from Study 3 for all the combinations for Female Caucasian and Female Class.

- With usage of demographic information: Male Class for 30m distance

- Male Class, rank-1 identification accuracy improved from 61\% (Only Study 1) (see in Table 7.3) to 76\% where Study 1 followed by Study 2 and Study 3 (see Fig. 7.4). 
- Male Class With Beard, the rank-1 identification accuracy improved from $76 \%$ to $82 \%$ (Study1 followed by Study 2 and Study 3), see in Table 7.3).

- Results are similar from Study 3 for all the combinations for Male Without Beard and Male Class.

For $90 \mathrm{~m}$ and $120 \mathrm{~m}$ distance there is a large improvement in the results for the data with demographic information. For $90 m$, the performance of the systems is improved from $30 \%$ to $41 \%$ for FA, $43 \%$ for MWB and $38 \%$ for MWOB. For $120 m$, the performance of the system is improved from $28 \%$ to $38 \%$ for FA, and $38 \%$ for MWB. For $30 m$ distance, without stratification for all the database the rank-1 identification accuracy is achieved $73 \%$ from the developed method (see in Table 7.1). It was determined that the better performance results are achieved after stratification e.g. the rank-1 identification accuracy is $75 \%$ for FA, $75 \%$ for MWOB and $82 \%$ for MWB.

The overall best results are achieved for FA, MWB and MWOB (soft biometric traits) for $30 \mathrm{~m}, 90 \mathrm{~m}$ and $120 \mathrm{~m}$ in comparison to all the database (without stratification) as illustrated in Table 7.2 For one of the soft biometric trait (FC), we were not able to achieve as good results as originally expected. A valid reason for these results is the type of stratum and there is a very small difference in the subject's appearance. For the $60 \mathrm{~m}$ distance, the results are very close, with and without using stratification. The developed methods including: image restoration, feature selection, decision level fusion and, finally, the selection of soft biometric traits (stratification of database) result in significant improvement in face identification results than available commercial FR matcher.

\subsection{Threats to Validity}

\subsubsection{Construct Validity}

In this study an image restoration scheme is developed to restore the good quality images for the database collected under challenging conditions in night time and at long standoff distances.

\section{Threats from Developed Study:}


- The developed system is based on wavelet based de-noising method to remove the noise and example based super-resolution method for image up-scaling. In a scenario, where the researchers want to restore the system on new or other existing methods, they need to redesign the system, otherwise the performance of the system will be affected.

\subsubsection{Internal Validity}

Based on the results, it is claimed that restored images improve the performance of face recognition system for with and without grouping of databases.

\section{Threats from Developed Study:}

- Performance of the developed scheme is dependent on the scaling factor for superresolution method and the type of window function for de-noising method i.e. Hamming, Hanning, Bartlett, Blackman, Kaiser, Chebwin, Butterworth, etc. To use the new window function, window size or scaling factor, researchers need to redesign the system, otherwise it may result in poor performance.

\subsubsection{Conclusion Validity}

Based on the results, it is determined that conclusions made from study 3 are justified. Based on face matching experiments for both the cross-distance and cross-spectral scenarios, the developed image restoration scheme improves the rank identification accuracy.

\section{Threats from Developed Study:}

- Testing the developed approach on a small dataset is a common threat. In this work, the developed approach is tested on the database collected under 4 standoff distances of 30 , 60, 90 and 120 meters.

\subsubsection{External Validity}

The validity is concerned about generalization. The main concern is up to what level or point a result or outcome be generalized to population, treatment variables and settings.

\section{Threats from Developed Study:}


- The developed image restoration scheme is working well for academic database with 103 subjects in NIR band. However, to make the system robust it needs to be tested on other databases e.g. thermal databases. Hence, results may be affected for the databases collected in other bands. 


\section{Chapter 8}

\section{Study 4: Statistical Analysis}

In this chapter, the statistical tests are implemented to find the statistical significance of incorporating developed image restoration and score level fusion schemes, as well as of the database stratification or demographic filtering in terms of gender, ethnicity, and facial hair. The effect of statistical significance study to FR performance is also discussed. The conducted set of statistical tests are represented by a pipeline which consists of selection of statistical test for the database, hypothesis test 1, hypothesis test 2 and hypothesis test 3 as shown in Fig. 8.1 What follows is a discussion on the hypothesis tests performed. The reasoning on the selection of particular tests on specific scenarios is investigated.

\subsection{Statistical Hypothesis Testing}

In this work, for FR studies the features are extracted using the LBP, Gabor and HOG feature descriptors. While performing face matching experiments, the distance scores are calculated using cosine similarity metric to compare faces in the normalized projected space, as described in Chapter 4 (section 4.1.3). These match scores are used as sample data to perform the statistical tests.

(i) Hypothesis Test 1: It is evaluated whether the fusion of match scores from three selected feature descriptors will have a statistically significant impact on the performance of face recognition system. 


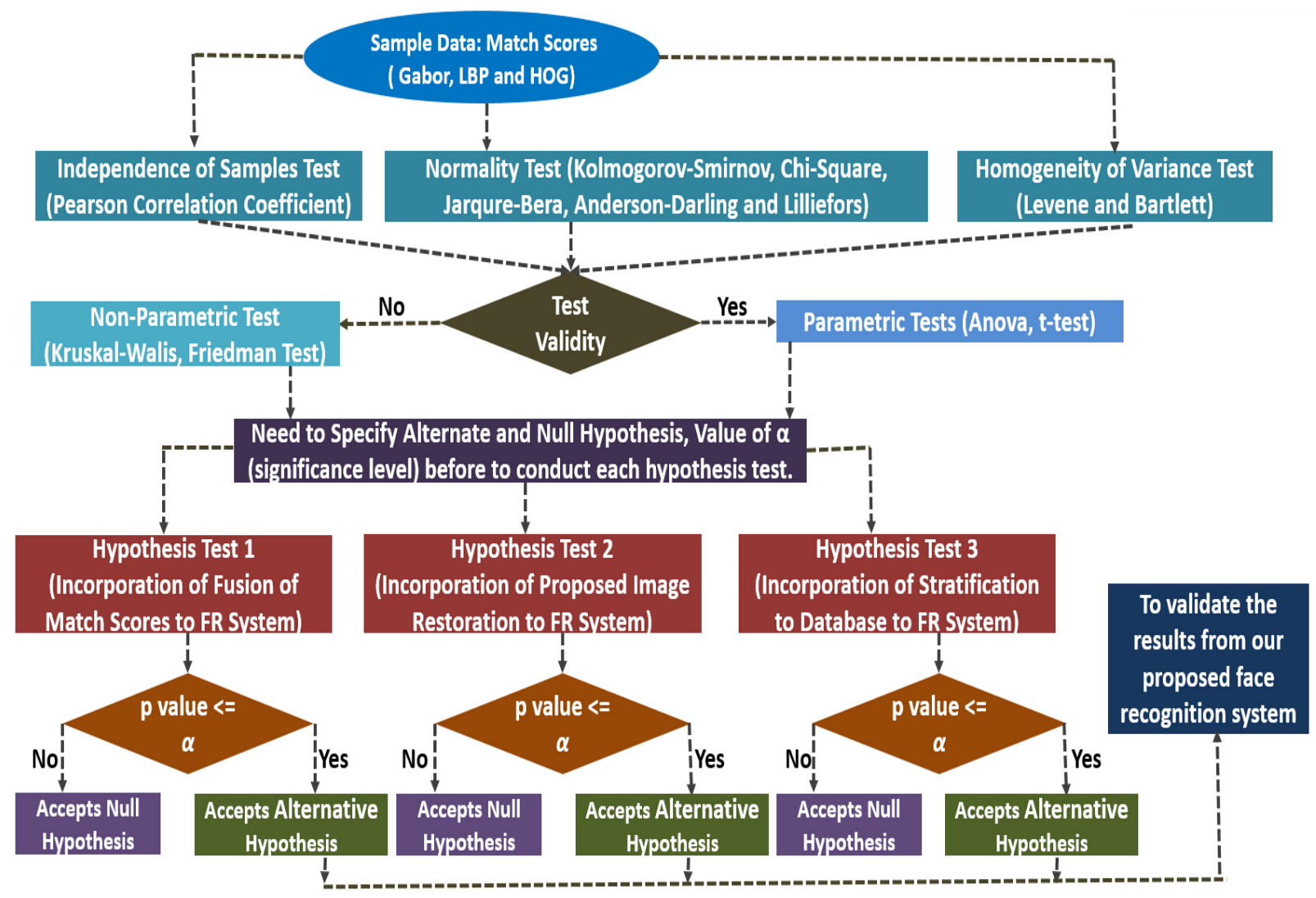

Figure 8.1: Pipeline for statistical significance tests.

(ii) Hypothesis Test 2: It is evaluated whether the developed image restoration algorithm will have a statistically significant impact on the performance of face recognition system.

(iii) Hypothesis Test 3: It is evaluated whether incorporating the database stratification into four strata including; Female Asian, Female Caucasian, Male with Beard and Male without Beard will have a statistical significant impact on the performance of face recognition system.

The designed null and alternative hypotheses are:

Null Hypothesis:

- Test 1: Performance results are identical for three selected feature descriptors and there is no statistical significant difference between performance results when each individual feature descriptor is used.

- Test 2: Performance results are identical for all the five pre-processing combinations (see Fig. 4.7) and no patterns are observed. 
- Test 3: Performance results are identical, with and without performing database stratification.

Alternative Hypothesis: Reject the null hypothesis, meaning there is a statistical significant difference between performance results for the selected hypotheses tests (i.e., Test 1, Test 2 and Test 3).

To find the statistical significance, it involves the terms such as $\mathrm{p}$ value and significance level and the detailed information is provided below:

- $p$ value is the probability of observing an effect, given that null hypothesis is true.

- Significance level $(\alpha)$ is the probability of rejecting null hypothesis when it is true. For example for a selected value of 0.05 for significance level, indicates a $5 \%$ risk of concluding that a difference exists when there is no actual difference.

- Statistically significant results are obtained, when $p$ value is less than or equal to $\alpha$.

- Additionally, skew-ness and existence of outliers was examined by using box-plots.

\subsection{Selection of Statistical Test}

Statistical tools are categorized into two main groups: parametric and non-parametric. Parametric tests assume that the population from which the samples are drawn has specific characteristics [102] including: (i) Samples are independent, (ii) Samples are drawn from normally distributed populations, and, (iii) Population variances are equal. On the other hand, nonparametric tests make assumptions related to the dependence and independence of samples but no assumptions is made about the population [103]. When the assumptions are violated (sample are normally distributed and independent samples), it leads to a threat to conclusion validity.

- The match scores from three selected feature descriptors are used as sample data to check the validity of the assumptions for the parametric tests.

- First, the independence/dependence of samples is tested. 
- Second, tests are performed to find the normal distribution.

- Finally, tests are performed to check the homogeneity of variances.

To conduct the tests, one factor (independent variable) with three groups or levels (match scores from three feature descriptors) is used. The dependent variable is the performance of the system. The statistical test for the sample data is selected based on the results from these tests. This is discussed in detail below.

\subsubsection{Independence/Dependence of Samples}

In this work the same subjects are selected to find the match scores from three feature descriptors, namely LBP (group1), Gabor (group2) and HOG (group3). To find the association with in and between the groups, the Pearson correlation coefficient (which is a measure of the linear dependence between two groups) is calculated. The results are presented in Table 8.1 The groups are always correlated to themselves (as the diagonal entities are 1). Group1 and Group2 are correlated but not as much as the other pairs of groups. However, it does not necessarily imply that the sample data (match scores) is independent.

Table 8.1: Correlation coefficient when Pearson test is used for match scores for Group 1 (Gabor), Group 2 (LBP) and Group 3 (HOG) for comb2.

\begin{tabular}{|c|c|c|c|}
\hline Groups & Group 1 & Group 2 & Group 3 \\
\hline Group 1 & 1.00 & 0.46 & 0.58 \\
Group 2 & 0.46 & 1.00 & 0.73 \\
Group 3 & 0.58 & 0.73 & 1.00 \\
\hline
\end{tabular}

\subsubsection{Statistical Test to find Distribution}

The normality of the match score distributions obtained by each feature descriptor is investigated. For this purpose the following five tests are used, the Kolmogorov-Smirnov test, Chisquare goodness-of-fit test, Jarque-Bera test, Anderson-Darling test and Lilliefors test [104]. In each experiment, the null hypothesis is that the distribution is normal. On the other hand, the alternative hypothesis rejects the null hypothesis and states that the distribution is not normal. 
Based on the results, it is determined that the returned value of $h$ is 1 and the value of $\mathrm{p}$ is less than the significance level $(\alpha=0.05)$ for all these tests, as presented in Table 8.2 . This means that the null hypothesis is not true (distributions are not normal). Therefore, the assumption that distribution for the database is normal is not valid. In Table 8.2, the results are shown for the Gabor descriptor. The same procedure is followed for LBP and HOG feature descriptors.

All these tests are performed on the selected five combinations as shown in Fig. 4.7. Here, the results for comb2 are presented. The distribution of match scores using the selected set of feature descriptors is shown in Fig. 8.2 (a)

Table 8.2: The distribution of match scores for features extracted using the Gabor descriptor when the second combination of pre-processing algorithms is used - see Fig. 4.7.

\begin{tabular}{|c|c|c|}
\hline Test & h & $\mathbf{p}$ \\
\hline Kolmogorov-Smirnov & 1 & 0 \\
Anderson-Darling & 1 & 0.001 \\
Jarque-Bera & 1 & 0 \\
Lilliefors & 1 & 0.005 \\
Chi-square goodness-of-fit & 1 & 0 \\
\hline
\end{tabular}

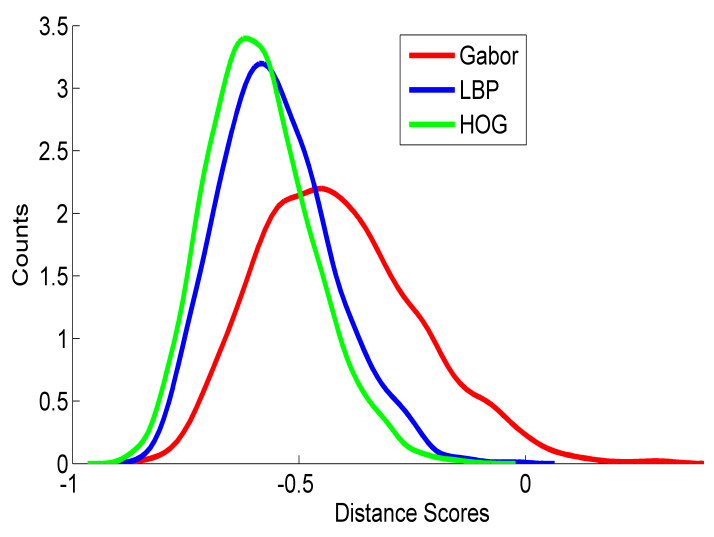

(a) Distribution plots

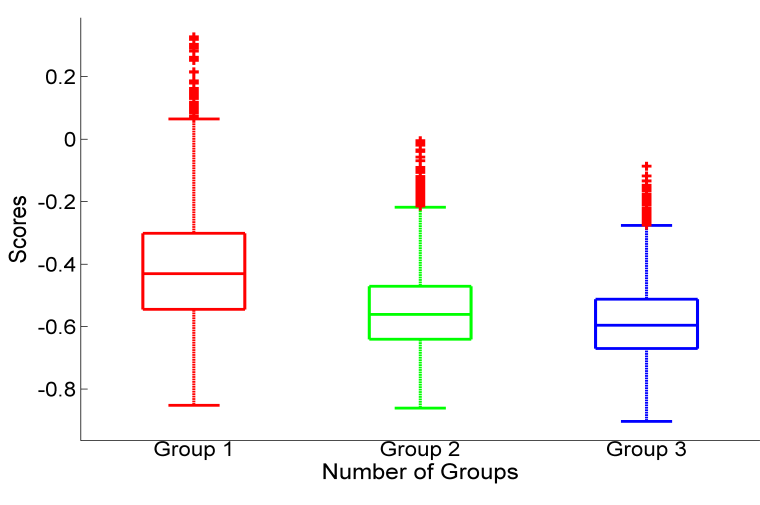

(b) Box-plots for distance scores

Figure 8.2: Validation check for parametric test.

\subsubsection{Statistical Tests to check Homogeneity of Variance}

In this test, it is determined whether the groups (i.e., the LBP, Gabor and HOG feature descriptors) have very different variances or not. Levene and Bartlett tests are used [105] in 
order to find the homogeneity of variance between the aforementioned group pairs.

Null hypothesis states that the columns of data or groups come from data with the same variance. The alternative hypothesis states that not all the groups have same variance and rejects the null hypothesis. The returned p-value is 0 (see Table 8.3 ), indicating that Levenes test rejected the null hypothesis that variances are equal across all the three columns. The alternative hypothesis is accepted as shown in Fig. 8.2 (b).

Table 8.3: Homogeneity of variance using Levene test for match scores for Group 1 (Gabor), Group 2 (LBP) and Group 3 (HOG) for comb2.

\begin{tabular}{|c|c|c|c|}
\hline Groups & Counts & Mean & Std Dev. \\
\hline Group 1 & 6400 & -0.41 & 0.18 \\
Group 2 & 6400 & -0.55 & 0.13 \\
Group 3 & 6400 & -0.59 & 0.12 \\
Pooled & 19200 & -0.52 & 0.14 \\
\hline \multicolumn{4}{|c|}{ p-value $=0$} \\
\hline
\end{tabular}

Based on results from statistical tests, all of the assumptions to implement the parametric tests are not valid for the multi-spectral, mutli-scenario database used for NIR to visible face matching. Thus, the non-parametric test (such as the Krushal-Walis and Friedman test) [106] are selected to perform the hypotheses tests 1,2 and 3. These methods are based on rank scores. The Krushal-Walis test assumes that the samples are independent for each level (group). Unfortunately, this assumption does not allow us to use the same sample data for testing (hypothesis), and thus, the Friedman's test is selected in this work. The evaluation results using the selected non-parametric test for the aforementioned hypothesis are provided in the experimental results section below.

\subsection{Statistical Hypothesis Test 1}

The Friedman test is implemented. The groups consist of match scores from three different feature descriptors, Gabor (Group 1), LBP (Group 2) and HOG (Group 3). The distance matrix is obtained from each feature descriptor, where each probe image (total 160 probe face images) is compared against all the gallery images (total 40 face images) and match scores are represented in rows for each probe $(160 \times 40)$. To reduce the dimensionality, feature vectors are 
Table 8.4: The match scores are converted into rank scores for each probe subject to perform the Friedman test. Here, the rank scores are represented for the first 9 samples (in total $6400 \times 3$ ), where rows represent rank scores for the first probe image when compared against the first 9 gallery images and columns represent the feature descriptors.

\begin{tabular}{|c|c|c|c||c|c|c|}
\hline Scores: & \multicolumn{2}{|c||}{ Match } & \multicolumn{3}{c|}{ Rank } \\
\hline Sample & Gabor & LBP & HOG & Gabor & LBP & HOG. \\
\hline s1 & -0.76 & -0.77 & -0.83 & 3 & 2 & 1 \\
s2 & -0.28 & -0.50 & -0.49 & 3 & 1 & 2 \\
s3 & -0.07 & -0.57 & -0.50 & 3 & 1 & 2 \\
s4 & -0.31 & -0.70 & -0.72 & 3 & 2 & 1 \\
s5 & -0.46 & -0.60 & -0.54 & 3 & 1 & 2 \\
s6 & -0.16 & -0.74 & -0.70 & 3 & 1 & 2 \\
s7 & -0.36 & -0.66 & -0.60 & 3 & 1 & 2 \\
s8 & -0.39 & -0.77 & -0.56 & 3 & 1 & 2 \\
s9 & -0.24 & -0.78 & -0.69 & 3 & 1 & 2 \\
\hline
\end{tabular}

represented into one column $(6400 \times 1)$. In total, the dimensionality is $(6400 \times 3)$, where column represents the number of feature descriptors used. These match scores are used as sample data.

The "match" scores are converted to "rank" scores (represented as columns) for each row based on scores from each descriptor, in Table 8.4 the results are presented for the first 9 samples.

In this experiment, the null hypothesis states that three groups have identical effects or it can be interpreted as that means are same. The alternative hypothesis states that there is a difference between the three groups. As shown in Table 8.5 .

- The chi-square distribution method is used to find the probability distribution.

- The value 0.05 is selected for $\alpha$.

- Second column shows the sum of square $(S S)$ between the groups using the ranks instead of match scores.

- Third column shows the degree of freedom $d f$ associated with each group.

- Fourth column is mean square (MS), which is ratio of SS/df.

- Chi-square static is represented in column five

- $p$ value for chi-square static is represented in last column of table. 
Table 8.5: Friedman and Post-hoc tests for match scores to find out the statistical significant difference between groups.

\begin{tabular}{|c|c|c|c|c|c|}
\hline \multicolumn{6}{|c|}{ Freidman Test } \\
\hline Source & SS & df & MS & Chi-sq & Prob $>$ Chi-sq \\
\hline Columns & 5233.64 & 2 & 2616.82 & 5233.64 & 0 \\
\hline Error & 7566.36 & 12798 & 0.59 & & \\
\hline Total & 12800 & 19199 & & & \\
\hline \multicolumn{6}{|c|}{ Post Hoc: Tukey - Kramer } \\
\hline Group1 & Group2 & $\begin{array}{l}\text { Lower } \\
\text { Limit }\end{array}$ & $\begin{array}{c}\text { Difference } \\
\text { group means }\end{array}$ & $\begin{array}{l}\text { Upper } \\
\text { Limit }\end{array}$ & $\begin{array}{c}\mathrm{p} \\
\text { value }\end{array}$ \\
\hline 1 & 2 & 0.78 & 0.82 & 0.86 & 0 \\
\hline 1 & 3 & 1.21 & 1.26 & 1.30 & 0 \\
\hline \multirow[t]{2}{*}{2} & 3 & 0.40 & 0.44 & 0.48 & 0 \\
\hline & $\begin{array}{c}\mathrm{m}=\text { Estimated values } \\
\text { of the means } \\
\text { for each group }\end{array}$ & $\begin{array}{l}\text { Standard } \\
\text { Errors }\end{array}$ & & & \\
\hline Group 1 & 2.6939 & 0.0125 & & & \\
\hline Group 2 & 1.8716 & 0.0125 & & & \\
\hline Group 3 & 1.4345 & 0.0125 & & & \\
\hline
\end{tabular}

The value of $p$ is less than $\alpha$ (significance level). Based on the results, it is concluded that results are significant and we can reject the null hypothesis that all the means are same and no patterns are observed.

The information provided from this test is, whether the samples are from same distribution and if there is a statistical significance between the groups. On the contrary, it could not tell us which specific groups are statistically different from each other. A post hoc test is performed to find out which specific groups are significant, including; Tukey, Bonferroni, Dunn-sidak, Fischers least significant difference and Scheffe test [107]. In Table 8.5, based on the post hoc test, the returned value of $p$ is 0 and is less than $\alpha$ (significance level) of 0.05 , and it is concluded that there is a significant difference between groups 1 and 2, 1 and 3 and 2 and 3.

To find the correlation, the Pearson correlation coefficient $(r)$ is calculated. As shown in Fig. 8.3, the groups are negatively correlated, which means that when the values for one group increase, they decrease for second group and vice versa. In Fig. 8.3, the first group (var1) represents the rank scores for Gabor features, group 2 (var2) represents for LBP and group 3 (var3) for HOG feature descriptor. The small value of $p$ (less than significance level $0.05)$, represents, that the correlation $R(i, j)$ is significant. Where, $i$ and $j$ represents the two 


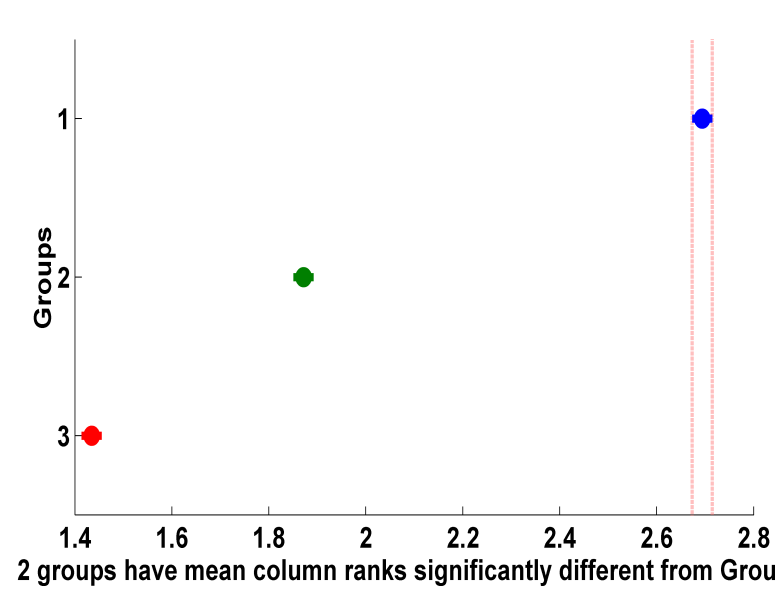

(a) Post-hoc test

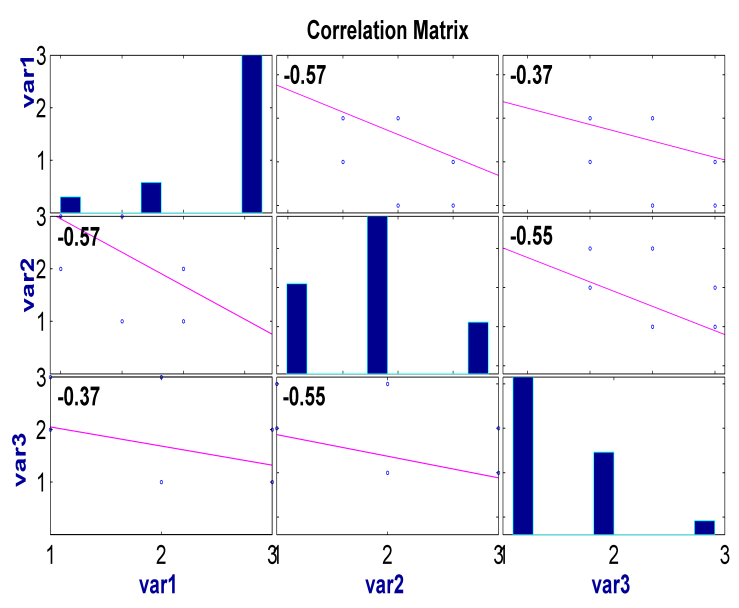

(b) Pearson correlation test

Figure 8.3: For Post-hoc test (Left), y-axis represents the three selected groups such as Group 1 (GaborBlue), Group 2 (LBP-Green) and Group 3 (HOG-Red) and x-axis represents the rank scores. The association between the groups is calculated using Pearson correlation test (Right).

different groups selected. The null hypothesis is rejected based on the statistical tests performed including, Friedman test, post-hoc test and correlation relationship. It is concluded that there is no statistical significance difference and the alternative hypothesis is accepted.

Fusion Studies: Based on [108, 109], when we combine the matchers that are negatively correlated, it may result in a significant improvement in performance than combining two strong biometric matchers [109]. To improve the overall performance, in this work, score level fusion schemes are adopted for the match scores achieved using three different feature descriptors. Pearson correlation is shown in Fig. 8.3 (var 1 for group 1, var 2 for group 2 and var 3 for group $3)$.

In this work, 8 different fusion scenarios based on sum and weighted fusion are investigated. Here, schemes $A 1, A 2$ and $A 3$ are without any fusion and are based on individual methods. For sum fusion method, the fusion of normalized distance scores, $z 1$ for Gabor/KLDA, $z 2$ for LBP/KLDA and $z 3$ for HOG/KLDA is performed using four different cases. When the weighted fusion method is used, the weights are assigned to the normalized scores based on the values that were achieved from the other independent methods tested. When the weighted fusion scheme is used, weights are automatically selected for each combination (combl to comb5). The fusion of normalized distance scores with assigned weights is performed using four different cases (Gabor-HOG, Gabor-LBP, LBP-HOG and Gabor-LBP-HOG). The final 
results are presented with rank-1 identification accuracy after the fusion for all of the four cases.

Based on the statistical results, best performance (rank-1 identification accuracy) is achieved for the fusion scheme $A 8$ (weighted fusion with green box) as shown in Fig. 8.4. The number and location of these outliers is also used for the selection of the best combination in terms of performance. The large number of these outliers for the distance scores in the range from 0.90 to 1.0 results in poor performance of the system. The rank-1 identification accuracy along with total number of outliers is represented in Table 8.6. The first three scenarios $(A 1, A 2, A 3)$ are performance of face recognition using individual feature descriptors without fusion. These three scenarios are not included in Table 8.6. Fusion scenarios are selected from $A 4$ to $A 11$ as shown in Fig. 8.4. The first four fusion schemes are based on sum fusion and rest on weighted fusion.

Table 8.6: Number of outliers and location of these outliers from box-plots for comb5.

\begin{tabular}{|c|c|c|c|c|c|}
\hline $\begin{array}{c}\text { Fusion } \\
\text { Scheme }\end{array}$ & $\begin{array}{c}\text { No. of } \\
\text { Outliers }\end{array}$ & $\begin{array}{c}\text { No. of } \\
\text { Outliers } \\
\text { (0.90 to 1.0) }\end{array}$ & Median & Std. Dev. & $\begin{array}{c}\text { FR Rank-1 } \\
\text { Identification Accuracy }\end{array}$ \\
\hline A4 & 45 & 5 & 0.38 & 0.15 & 0.70 \\
A5 & 60 & 6 & 0.39 & 0.15 & 0.65 \\
A6 & 75 & 6 & 0.35 & 0.14 & 0.60 \\
A7 & 50 & 9 & 0.39 & 0.15 & 0.66 \\
A8 & $\mathbf{4 5}$ & $\mathbf{3}$ & $\mathbf{0 . 3 7}$ & $\mathbf{0 . 1 4}$ & $\mathbf{0 . 7 1}$ \\
A9 & 71 & 11 & 0.39 & 0.15 & 0.65 \\
A10 & 67 & 4 & 0.35 & 0.14 & 0.59 \\
A11 & 42 & 4 & 0.36 & 0.13 & 0.66 \\
\hline
\end{tabular}

From Table 8.6, the combination $A 8$ is selected, as this has less number of outliers, considering factors such as the location of outliers (more are in numbers in region from 0.0 to 0.2 , less in numbers in range of 0.9 to 1.00$)$, median and value of standard deviation.

Based on the statistical significance tests, the best combination is selected $A 8$ (Table 8.6), and based on face recognition, best results for comb5 are achieved from $A 8$ as represented in Table 8.6 (in the last column with FR rank-1 identification accuracy). Based on the results, it is concluded that analysis results from statistical tests (hypothesis test 1 ) are validated for all the five combinations with face recognition experiments. 


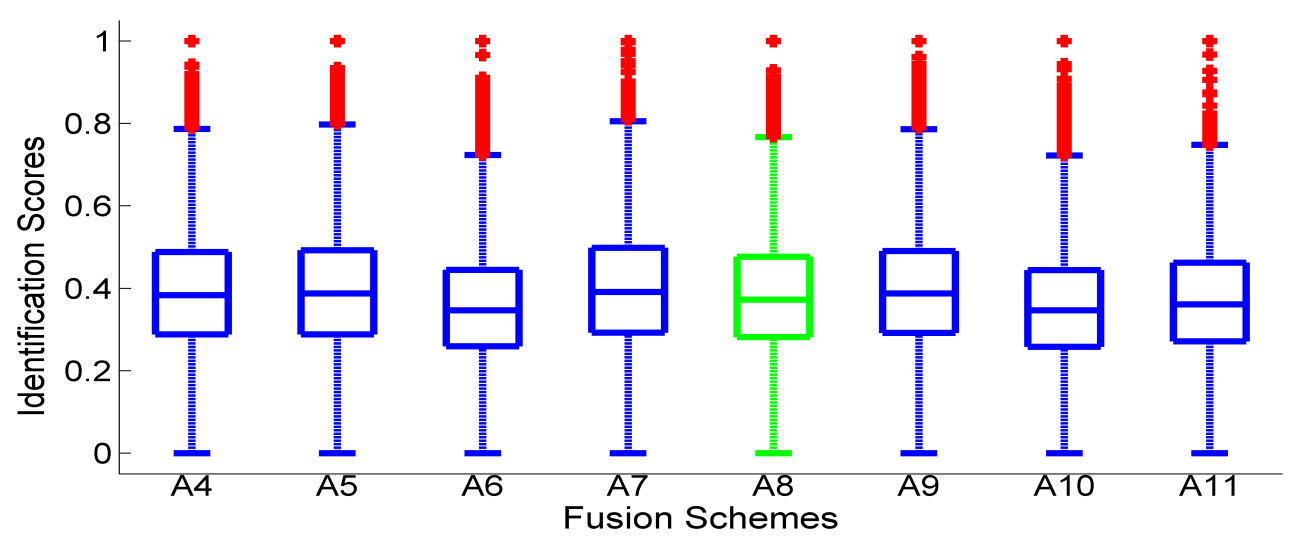

Figure 8.4: Boxplot for the distance scores when images are matched using different fusion schemes.

\subsection{Statistical Hypothesis Testing 2}

This hypothesis test is performed to test the significance of incorporating developed image restoration to FR system.

Null Hypothesis: The performance results are identical for all the five selected pre-processing combinations and no patterns are observed.

AlternativeHypothesis: Reject the null hypothesis and patterns are observed.

\section{- Cross-spectral matching results at distance of 30m:}

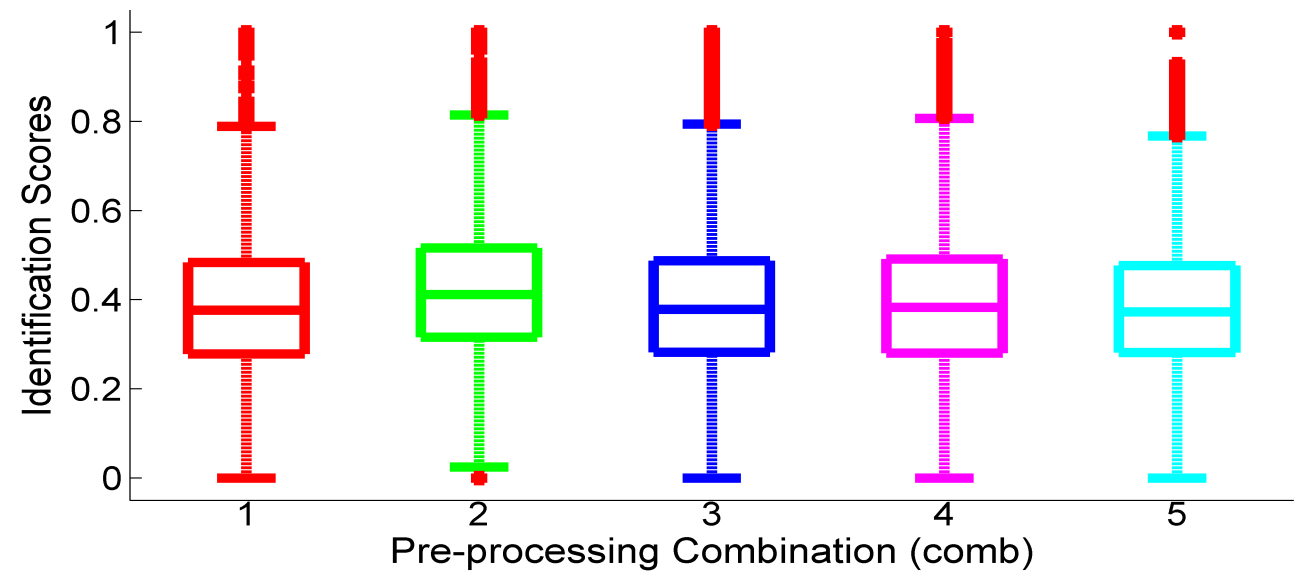

Figure 8.5: Box-plots for the distance scores using combination of pre-processing techniques.

For probe data (without stratification) collected at a distance of $30 \mathrm{~m}$, two statistical tests are applied for each combination, (with selected fusion scheme from hypothesis test 1 for comb5 see Fig. 8.4 with green box), first test is to find the distribution and second is to find the 
significance difference between the variance.

Based on distribution test, returned value of $h$ is 1 , and thus, the null hypothesis that distribution is normal is rejected. For the variance test, the returned value of $p$ is 0 , and thus, the null hypothesis that all columns have same variance is rejected.

Five combinations are considered for the pre-processing studies (see Fig. 4.7), for combl, $P N$ is applied directly to $G N$ raw images and for comb2 to $c o m b 5, P N$ is applied to the restored face images from developed method. From Table 8.7, it is concluded that the similar number of outliers, median and deviation value is achieved for comb1 and comb5. For comb2, the number of outliers in distance score lie in the range from 0.0 to 0.2 , which results in better performance (see Fig. 8.5 green box). On the other hand, there are larger number of outliers for comb3 and comb4, which results in poor performance of face recognition system. In the last column of the Table 8.7, the rank-1 identification accuracy for the FR system is presented. Based on the results, it is concluded that FR results are aligned with the conclusions drawn from the statistical significance test. Finally, based on results from statistical analysis and achieved rank-1 identification accuracy from face recognition system, combl is selected for the database at $30 \mathrm{~m}$.

Table 8.7: Selection of Pre-processing combination (for the face images at 30m) based on results from box-plots. Performance is affected by median, standard deviation, number of outliers and location of these outliers.

\begin{tabular}{|c|c|c|c|c|c|}
\hline $\begin{array}{c}\text { Pre-processing } \\
\text { combination }\end{array}$ & $\begin{array}{c}\text { No.of } \\
\text { Outliers }\end{array}$ & $\begin{array}{c}\text { No.of } \\
\text { Outliers } \\
(\mathbf{0 . 9 0} \text { to 1.0) }\end{array}$ & Median & Std. Dev. & $\begin{array}{c}\text { FR Rank-1 } \\
\text { Identification } \\
\text { Accuracy }\end{array}$ \\
\hline 1 & 41 & 11 & 0.38 & 0.14 & 0.73 \\
2 & 51 & 15 & 0.41 & 0.15 & 0.73 \\
3 & 61 & 18 & 0.38 & 0.15 & 0.69 \\
4 & 57 & 12 & 0.38 & 0.16 & 0.67 \\
5 & 45 & 3 & 0.37 & 0.14 & 0.70 \\
\hline
\end{tabular}

\subsection{Statistical Hypothesis Test 3}

In this the statistical significance of incorporating the stratified database for face recognition experiments is evaluated. Null hypothesis states that performance results are identical for with 
and without stratification of database. On the other hand, alternative hypothesis states that stratification results are significant.

Table 8.8: Selection of pre-processing combination (for stratified database) based on results from boxplots. Performance is affected by median, standard deviation, number of outliers and location of these outliers.

\begin{tabular}{|c|c|c|c|c|c|}
\hline $\begin{array}{c}\text { Pre-processing } \\
\text { combination }\end{array}$ & $\begin{array}{c}\text { No.of } \\
\text { Outliers }\end{array}$ & $\begin{array}{c}\text { No.of } \\
\text { Outliers } \\
\text { (0.90 to 1.0) }\end{array}$ & Median & Std. Dev. & $\begin{array}{c}\text { FR Rank-1 } \\
\text { Identification } \\
\text { Accuracy }\end{array}$ \\
\hline Female Caucasian & 11 & 2 & 0.28 & 0.19 & 0.53 \\
1 & 1 & 1 & 0.39 & 0.20 & 0.50 \\
2 & 12 & 3 & 0.30 & 0.19 & 0.50 \\
3 & 4 & 2 & 0.32 & 0.18 & 0.53 \\
4 & $\mathbf{2}$ & $\mathbf{2}$ & 0.30 & 0.18 & $\mathbf{0 . 5 3}$ \\
$\mathbf{5}$ & 4 & 1 & 0.26 & 0.18 & 0.53 \\
Female Asian & & 0 & 0.34 & 0.25 & 0.70 \\
2 & 0 & 1 & 0.37 & 0.20 & 0.66 \\
3 & 1 & 2 & 0.34 & 0.20 & 0.62 \\
4 & 2 & $\mathbf{0}$ & 0.39 & 0.21 & $\mathbf{0 . 7 5}$ \\
\hline
\end{tabular}

The best combination is selected, considering the factors with minimum number of outliers, minimum value of median and deviation. For example, for female Caucasian stratum as represented in Table 8.8 , the minimum number of outliers are achieved from comb2 with maximum value for median and standard deviation. For combl, the value for median and deviation is lowest, on the contrary with large number of outliers. Finally, the comb5 is selected as the best combination (for Female Asian - FA and Female Caucasian - FC) based on number of outliers, variation in median and standard deviation. To test the validity of results when using the statistical tests, the results are compared with achieved results from the face recognition studies. The achieved performance result are better when comb5 is selected - as represented in the last column of the Table 8.8. Based on the results, it is concluded that FR results are aligned with the conclusions drawn from the statistical significance test. 
Table 8.9: Rank-1 identification accuracy results for ALL Data without any image restoration, fusion and stratification.

\begin{tabular}{|c|c|c|c|}
\hline Features & LBP/KLDA & Gabor/KLDA & HOG/KLDA \\
\hline Only GN & 0.45 & 0.20 & 0.50 \\
\hline
\end{tabular}

\subsection{Conclusion from Study 4}

In this work, the impact of the statistical hypothesis tests is studied for the purpose of improving rank-1 identification accuracy results for the images captured in heterogeneous environments, before conducting the face matching experiments. Based on the results for the selection of statistical test, it is concluded that:

- Friedman test (non-parametric) is the most suitable for the database collected in this work.

- Three statistical hypothesis tests are performed and based on the results the null hypothesis is rejected. The significance of incorporating the fusion scheme, the image restoration process and stratification of the database, before conducting face matching studies is presented.

- Statistical hypothesis test results are aligned with face identification results. The maximum rank-1 identification accuracy is 50\% (HOG) for ALL Data (without any image restoration, fusion and stratification) as represented in Table 8.9. The rank-1 identification accuracy improved from $50 \%$ to $71 \%$ for image restoration, to $73 \%$ based on the fusion of scores and to $75 \%$ for the stratification of the database (FC class).

- Face matching experiments are verified with the results from the statistical analysis study. 


\section{Chapter 9}

\section{Multi-Scenario and Multi-Wavelength}

\section{SWIR based Facial Recognition System}

When the face database collected under challenging conditions in the SWIR band, it results into poor performance of the recognition system. To deal with this problem, one of the solution is the data filtering before conducting face matching experiments. The main challenge is the automatic image filtering when using the SWIR database that is composed of face images captured under multiple scenarios, including, data captured indoors, outdoors, with or without tinted glass panels placed in front of a subject's face or even when active illumination at 1550 nm was on or off. Another challenge was the automatic classification of multi-wavelength database into individual wavelengths. The multi-wavelength data was collected at five different SWIR wavelengths ranging from $1150 \mathrm{~nm}$ and up to $1550 \mathrm{~nm}$ in increments of $100 \mathrm{~nm}$ (SSMW database) as discussed in the Chapter 4. To address this problem, a CNN based classification framework is developed which is, scenario-dependent and sensor adaptable. The focus of this chapter is the implementation of the developed convolutional neural network for image filtering and to determine whether the usage of image filtering can enhance recognition performance. The classification and face matching was performed for two different challenging databases (multi-scenario and multi-wavelength) collected in our lab. An overview of the developed approach is represented in Fig. 9.1. 


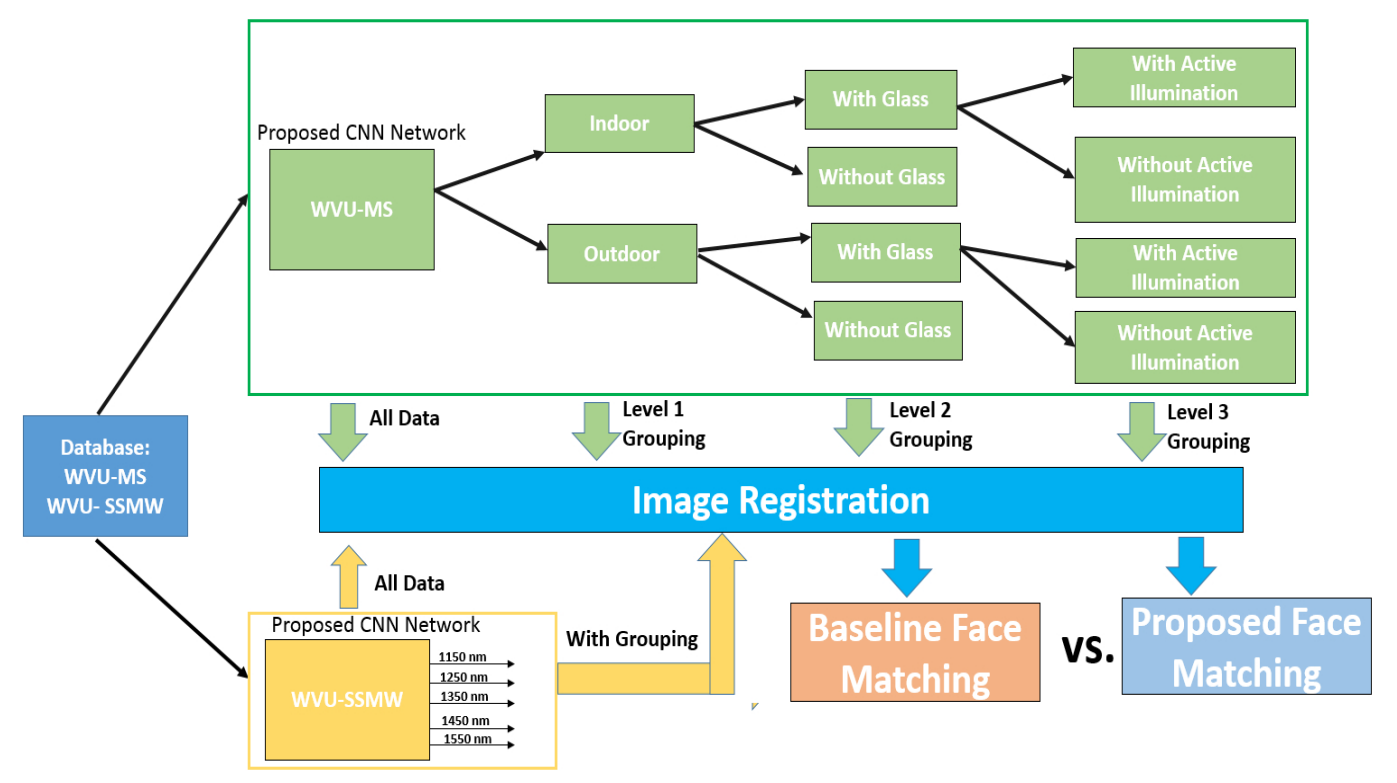

Figure 9.1: Overview of the developed data filtering network and face recognition system.

\subsection{Classification and Face Matching for Multi Scenario Database}

\subsubsection{Classification from Developed CNN Architecture}

The CNN network (as discussed in Chapter 4) is applied to perform the situation classification as follows:

- Level 1 classification, where the data collected under un-controlled conditions is used to classify into an indoor or outdoor class.

- Level 2 classification, a set of experiments are performed, where both indoor and outdoor face images are further classified into a with glass or no glass category.

- Level 3 classification, experiments are performed, where the level 2 face images are finally classified into with or without active illumination categories, before FR algorithms are used, as shown in Fig. 9.2

To perform the face matching experiments, the developed score level fusion based on MFSDF scheme (from study 1) is applied, and finally, the results are compared with baseline face matchers. Furthermore, based on a set of experiments, it is shown that the rank-1 identification accuracy obtained from grouping of the data and developed face matching approach results in higher recognition performance across all the scenarios. 


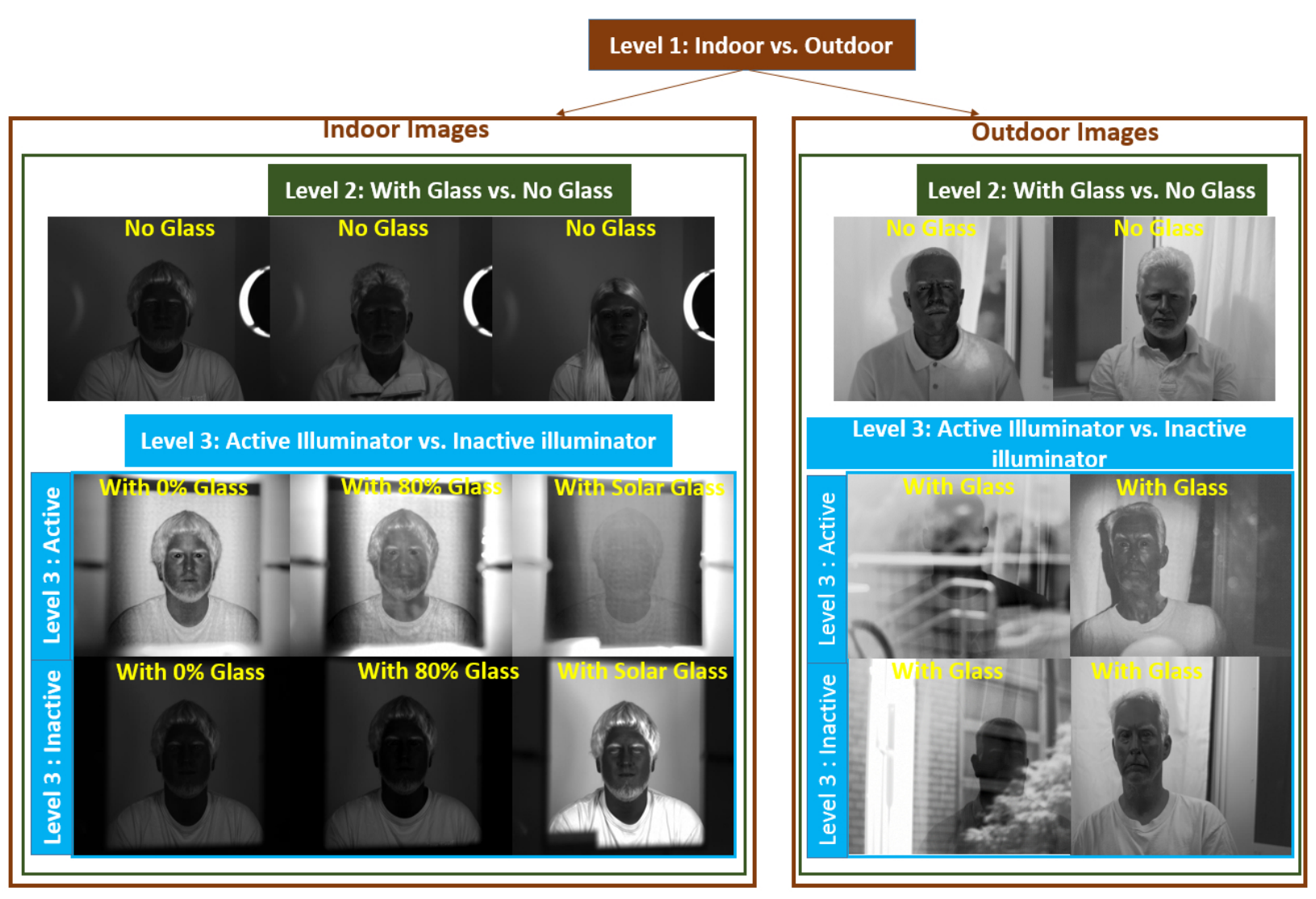

Figure 9.2: An overview of the developed hierarchical classification approach when using SWIR face images captured under various challenging conditions.

\subsubsection{Training and Testing}

There is no pre-trained data available for this challenging database i.e. with tinted glass (0\%, 80\% and Solar-cool tinted glass panels) and active illuminator in the SWIR band. To address this problem, WVU-MS database is selected to train and test the network (with frontal view). The models are trained on our database for each level (Level 1 to Level 3) and the model parameters were empirically optimized (e.g. epoch, momentum value set to 0.92 , batch size set to 100 and learning rate set to 0.002 ) to perform the level 1, 2 and 3 classification more accurately.

- Level 1 Classification Challenge: Indoor vs. Outdoor: To train the CNN network, the data is divided into different set sizes $(20 \%, 40 \%$ or $60 \%)$ for training, while rest of the data is used for testing. This process is repeated five different times for each set, using random selection (without any overlap).

Epoch Selection: To select an epoch value that results in good performance results, a number 


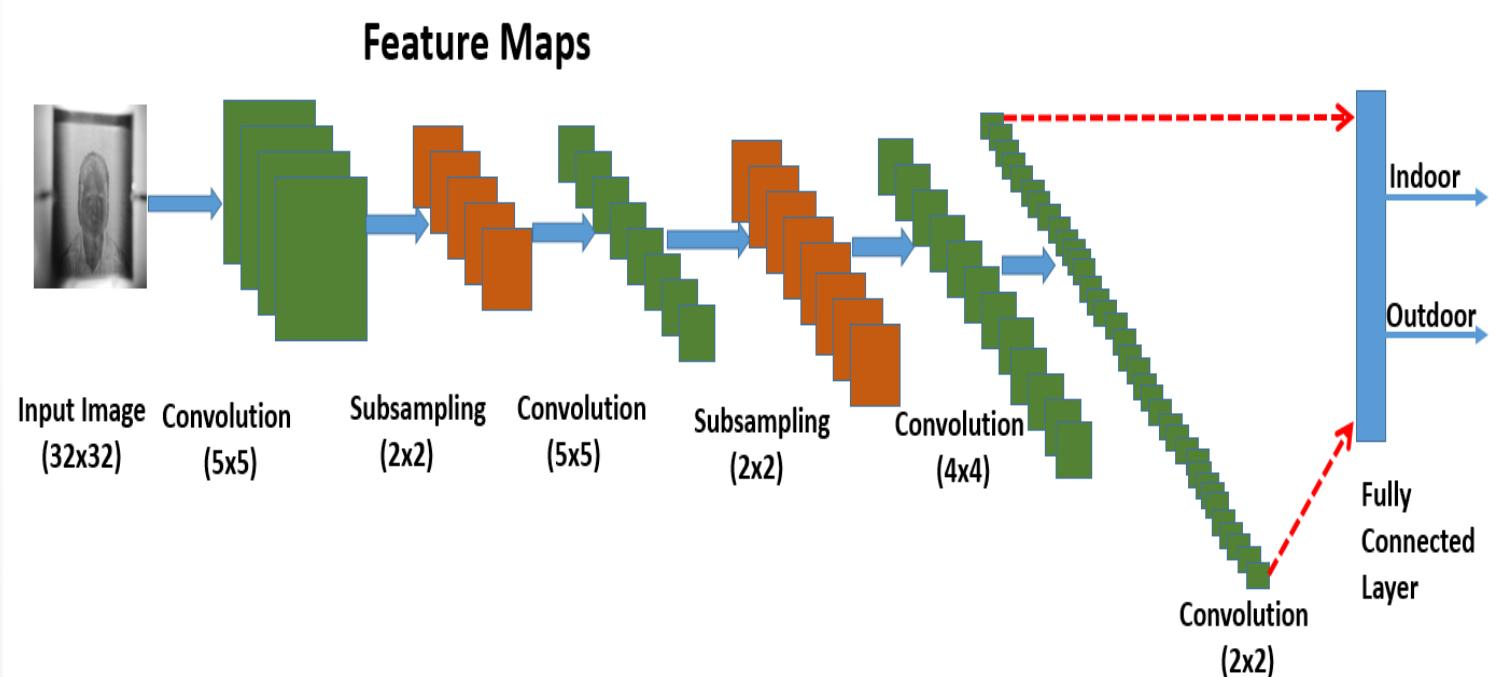

Figure 9.3: An overview of the developed CNN architecture.

of classification experiments are performed with a pre-defined set of epoch values, namely $4,8,12, \ldots, 52$. To better illustrate the variation in the performance results, see the boxplots in Fig. 9.4(a),

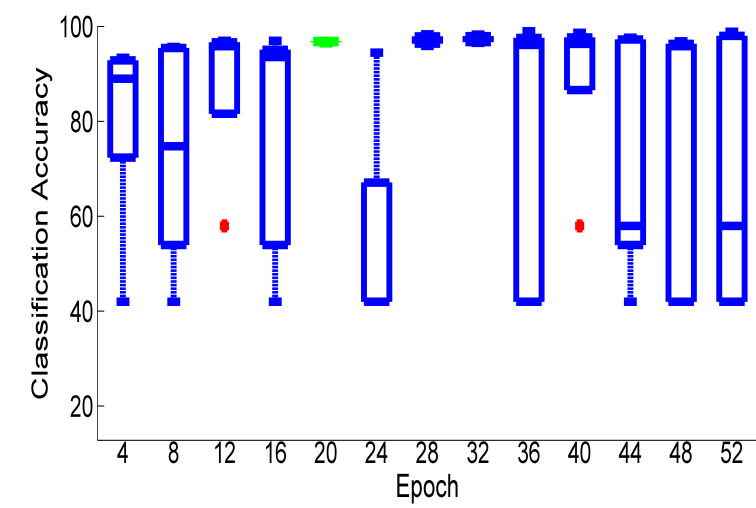

(a) Epoch Selection

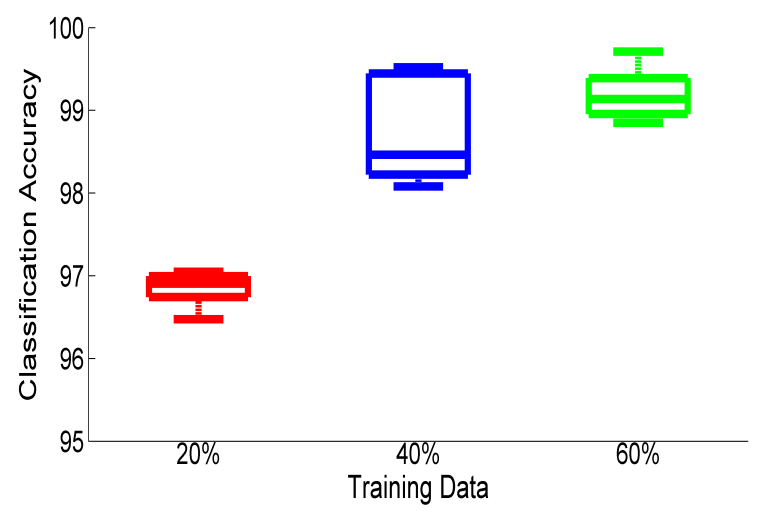

(b) Classification results

Figure 9.4: Level 1 (Indoor vs. Outdoor): Classification accuracy results with a selected set of epoch and training sets for CNN. Each boxplot is based on results from 5 randomly selected training and testing sets.

Based on the mean and variance plots, it is determined that the best classification results are achieved for the epoch value of 20 . The same set of experiments are repeated, when $40 \%$ and $60 \%$ of the data is used for training and the rest of the data is used for testing. It is concluded an epoch value of 20 results in the best performance, for all the \% of data values used for training. 
Classification Results: Based on mean and variance plots, the classification results are similar for $20 \%, 40 \%$ and $60 \%$ training sets and the classification accuracy reaches greater than $95 \%$ as shown in Fig. 9.4(b) Finally, a 20\% training set is selected, as it offers the least computational cost. The classification results for all five sets are presented in Table 9.1

- Level 2 Classification Challenge: No Glass vs. With Glass: For Indoor class (labeled

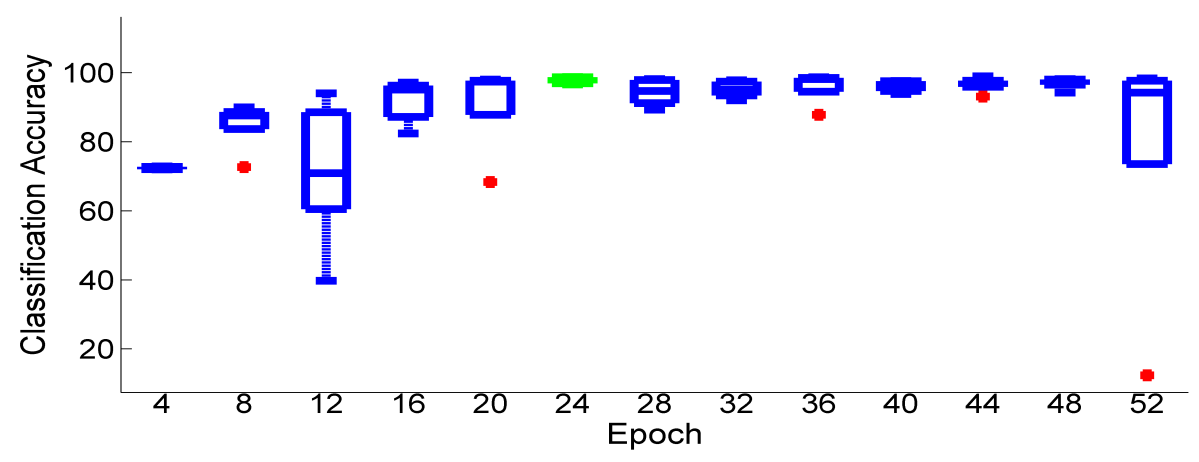

(a) Epoch Selection

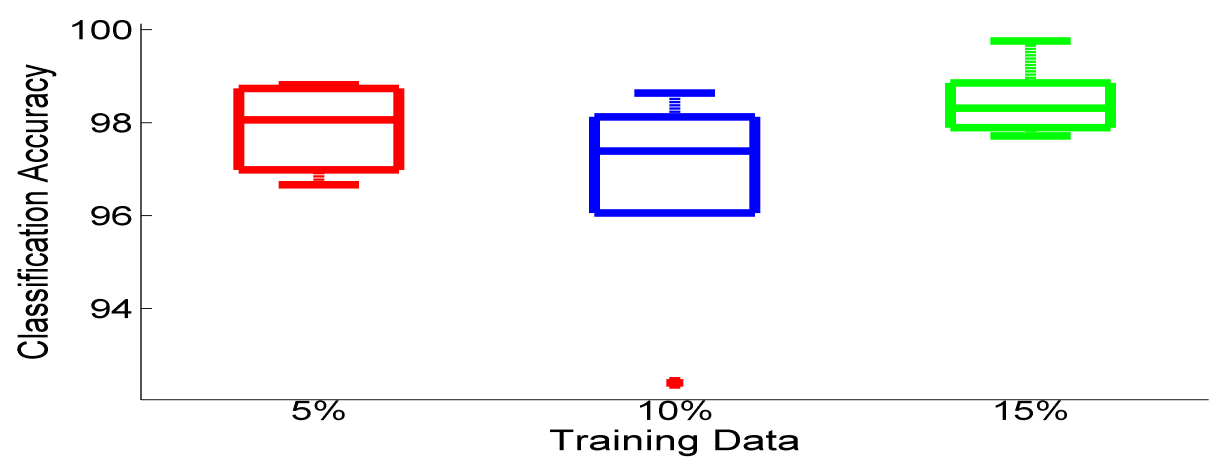

(b) Indoor Class

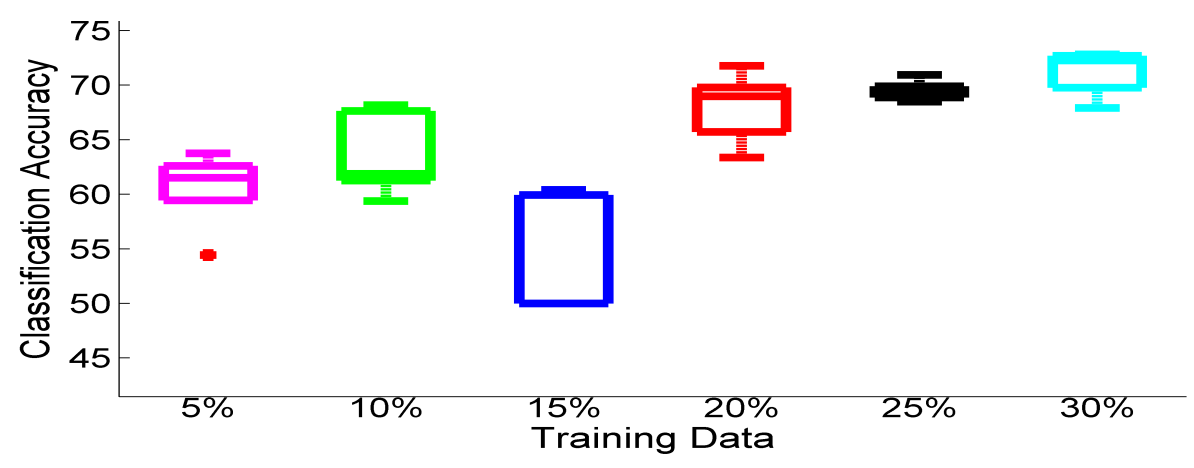

(c) Outdoor Class

Figure 9.5: Level 2 (With vs. No Glass): Classification results.

images from Level 1 classification), to train the CNN network, the data is randomly divided, 
based on training set sizes of 5\%,10\% and $15 \%$, while rest of the data is used as testing to evaluate the performance. For Outdoor class, data is randomly divided, based on training set sizes of $5 \%, 10 \%, \ldots, 30 \%$. This process was repeated five times. The variation in the selection of training sets between the indoor and outdoor class is due to image quality, where for the indoor class better performance results are achieved with a training set of $5 \%$.

Epoch Selection: First, experiments are performed for the indoor face data, where the aim was to classify them into with glass or no glass images (see Figure 1). To select an epoch value, performed the same set of experiments as described in Section 9.1. Based on mean and variance plots, the best classification results are achieved for an epoch value of 24 , for a training set of 5\% as shown in Fig. 9.5 (a). The same set of experiments are repeated, when selected the 10\% and $15 \%$ data for training.

Classification Results: Based on mean and variance plots, the classification results are similar for $5 \%, 10 \%$ and $15 \%$ training sets and the classification accuracy reaches greater than $95 \%$ as shown in Fig. 9.5(b). Finally, a 5\% training set is selected (the most challenging scenario).

Second, experiments are performed for outdoor face data, where the aim was to classify them into with glass or no glass categories (see Fig. 9.2). For the outdoor data category, 5\%, $10 \%, \ldots, 30 \%$ data is used for training and the rest for testing. Based on mean and variance plots, the best classification results are achieved for an epoch value of 44 , for training set size tested ranging from $5 \%$ to $30 \%$. The best classification results are achieved when $25 \%$ of data is used for training as shown in Fig. 9.5. (c).

- Level 3 Classification Challenge: With or Without Active Illumination: For this level of classification, the CNN network is trained as follows. The data is randomly divided based on training set sizes of $5 \%, 10 \%$ and $15 \%$, while rest of the data is used for testing. For the outdoor, with glass face dataset (labeled images from Level 2), the data is randomly divided into the training set sizes of $5 \%, 10 \%, \ldots, 30 \%$.

The same set of experiments are performed as described in Section 9.1 for epoch selection. Based on the results for the indoor class, the epoch value of 20 is selected when $5 \%$ of data is used for training, as shown in Fig. 9.6(a). Based on the results for the indoor class, it is concluded that the best performance results are achieved when $15 \%$ data is used for training and 


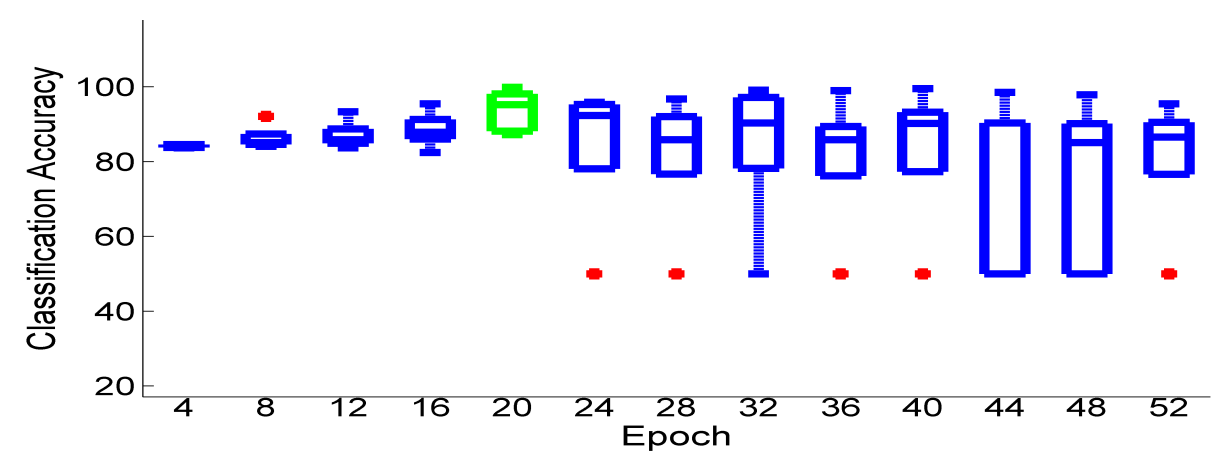

(a) Epoch Selection

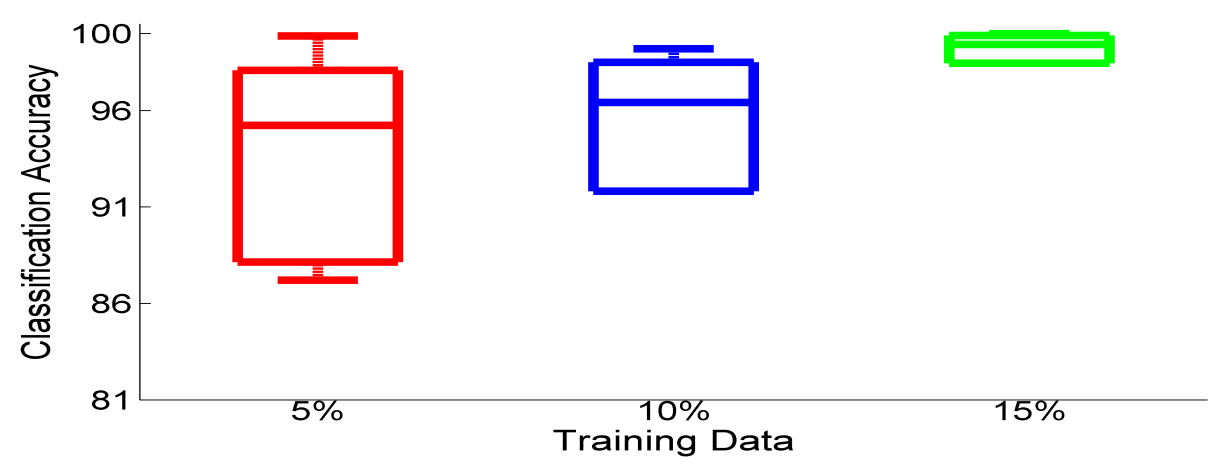

(b) Indoor Class

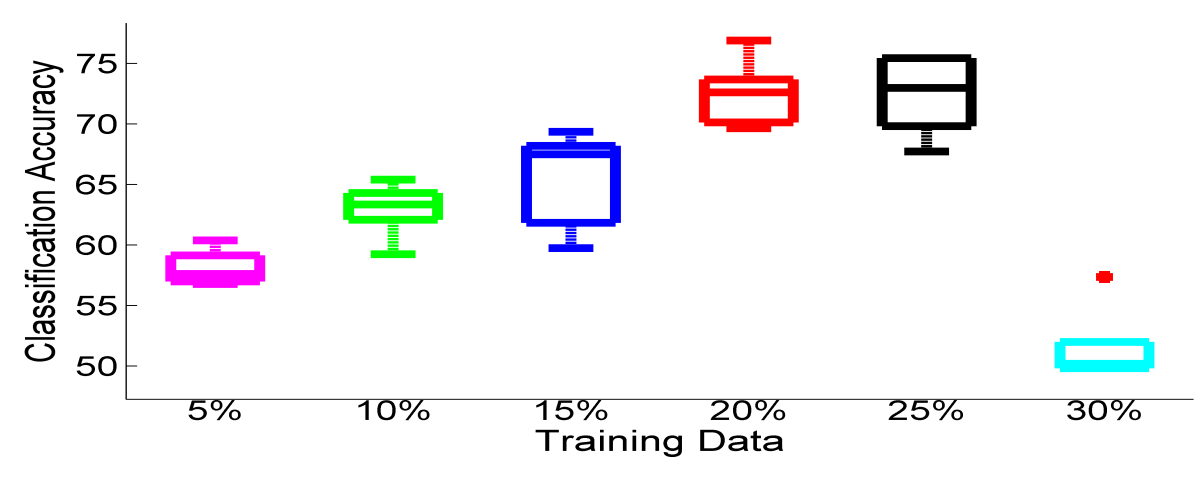

(c) Outdoor Class

Figure 9.6: Level 3 (With vs. Without Illumination): Classification results.

rest of the data is used for testing. The accuracy reaches greater than $98 \%$ for the classification as shown in Fig. 9.6(b). For the outdoor class, the best performance results are achieved when $20 \%$ data is used for training and rest of the data for testing. Please see classification results shown in Fig. 9.6(c).

The examples for the correctly classified (true positive) class for all the levels are provided in Fig. 9.7 and mis-classified images in Fig. 9.8 


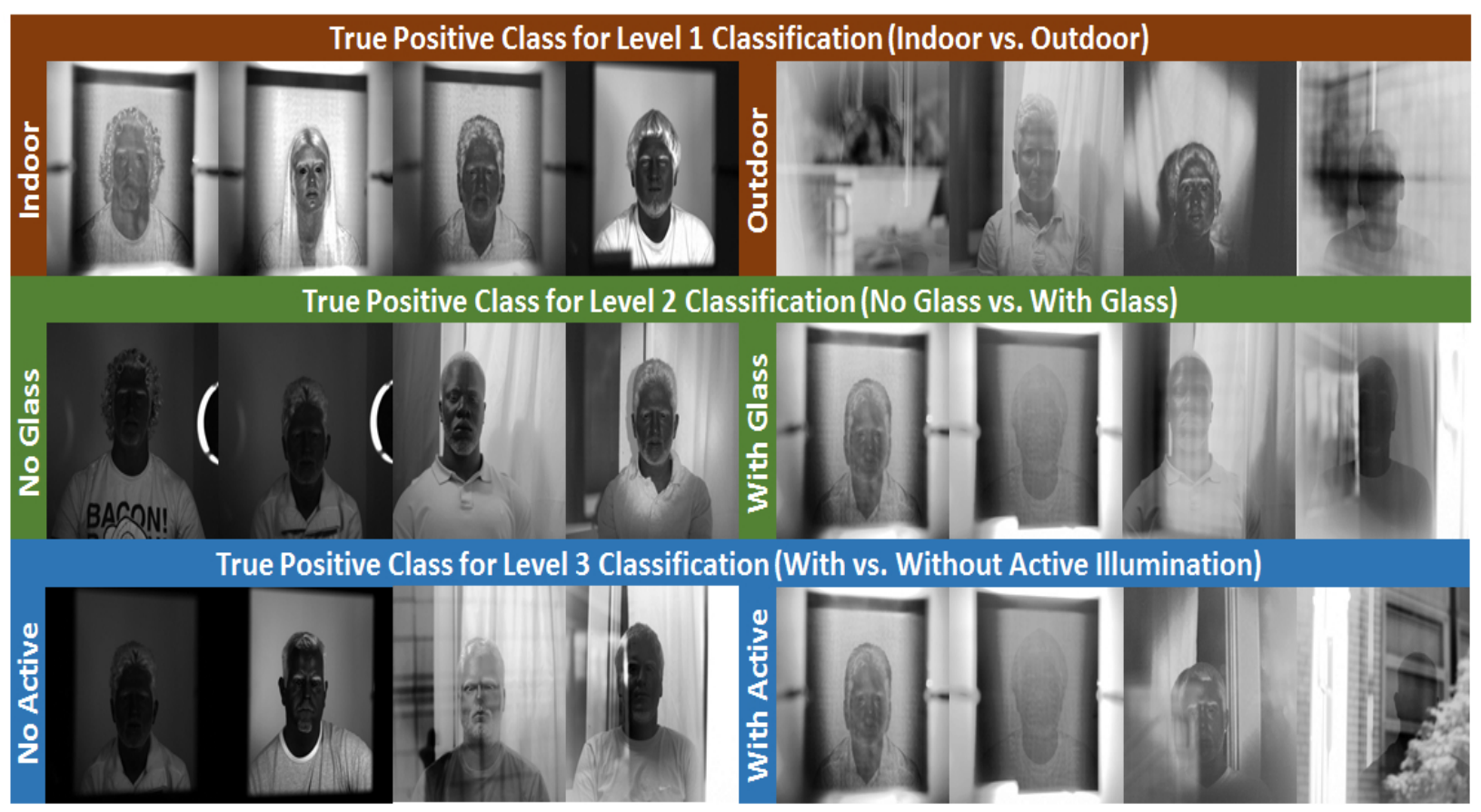

Figure 9.7: True positive examples from the developed CNN based classification approach. First row represents the correctly classified images from the level 1 classification into indoors or outdoors class. Second row for the level 2 classification into no glass or with glass. The first two images on the left represent classification for the indoor class into no glass and the other two images for the outdoor class into no glass. On the right side, the first two images represent the indoor class into with glass and the other two images for the outdoor glass into with glass. Third row represents the correctly classified images from the level 3 classification into with and without active illumination.

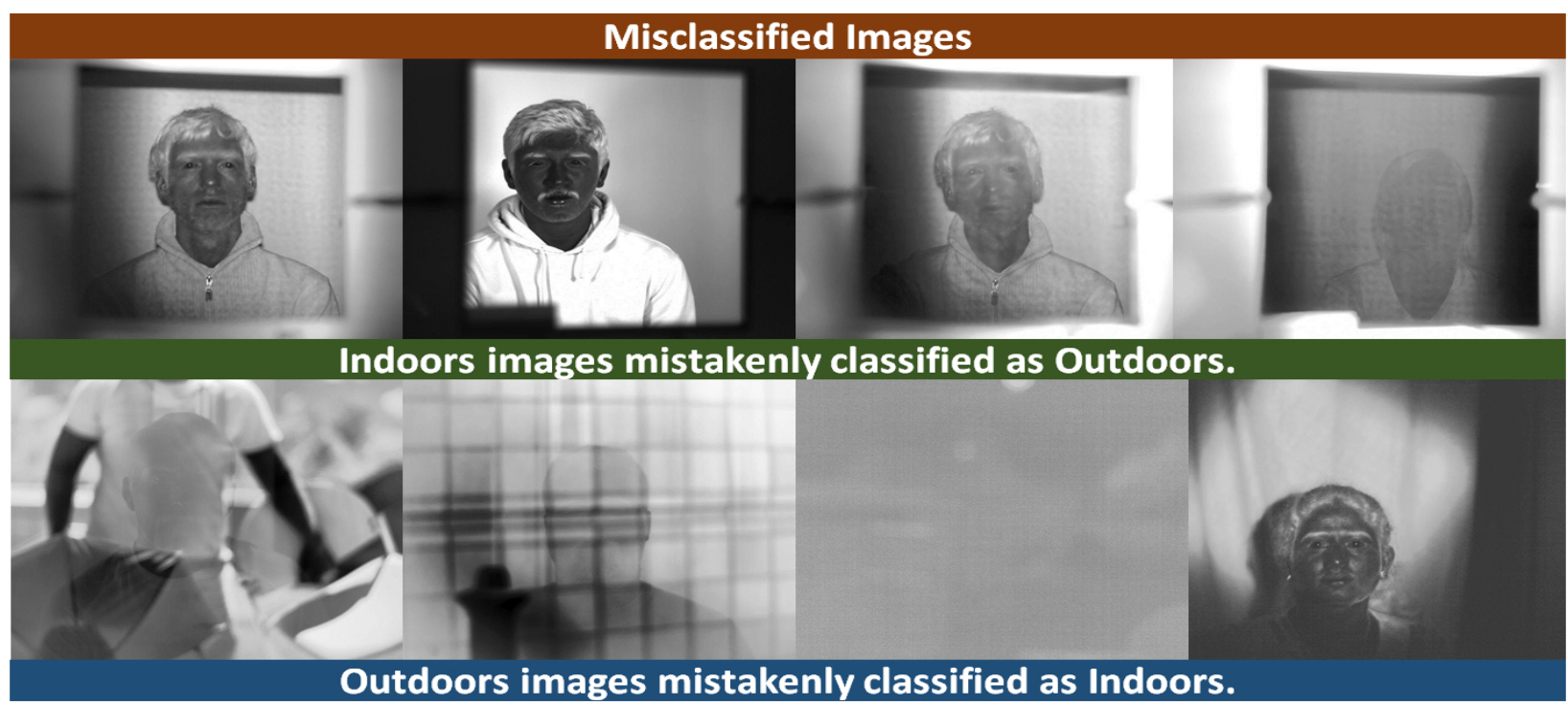

Figure 9.8: Misclassified images in level 1 classification. Top row: Indoor class images are falsely labeled as outdoor class. Bottom row: Outdoor images are falsely labeled as indoor. 
Table 9.1: Hierarchical classification results from CNN: randomly selected training sets.

\begin{tabular}{|c|c|c|c|c|c|}
\hline Datasets & set 1 & set 2 & set 3 & set 4 & set 5 \\
\hline \multicolumn{5}{|c|}{ Level 1: Indoor vs. Outdoor } \\
Train 20\% & 96.91 & 96.84 & 96.48 & 96.98 & 97.05 \\
Train 40\% & 98.46 & 98.27 & 99.52 & 98.08 & 99.42 \\
Train 60\% & 99.14 & 99.28 & 98.99 & 99.71 & 98.85 \\
\hline Level 2: With Glass vs. No Glass (Indoor) \\
Train 5\% & 96.67 & 98.71 & 98.82 & 97.10 & 98.07 \\
Train 10\% & 97.39 & 97.96 & 92.40 & 98.64 & 97.28 \\
Train 15\% & 99.76 & 98.32 & 97.72 & 98.56 & 97.96 \\
\hline Level 3: With vs. Without Illuminator (Indoor) \\
Train 5\% & 99.88 & 87.21 & 88.47 & 95.24 & 97.49 \\
Train 10\% & 96.45 & 99.21 & 98.28 & 95.90 & 79.63 \\
Train 15\% & 100.00 & 98.46 & 99.86 & 98.46 & 99.44 \\
\hline
\end{tabular}

\subsubsection{Cross-scenario Face Matching Results}

In cross-scenario FR studies, the system performance is evaluated using academic and the developed (MFSDF operator based on fusion scheme) face matchers. Intra-spectral (SWIR vs. SWIR) and cross-spectral (VIS vs. SWIR) face matching experiments are conducted. The main steps of face recognition system include, face normalization (neutral faces) and then identification followed by feature extraction and matching.

Two sets of face recognition experiments are performed using the texture based face matchers. First, experiments are performed with the original FR system, namely when no grouping of the database (level 1, level 2 and level 3) is used. Without grouping, all data is used to perform the face matching experiments. Second, experiments are conducted to determine whether the usage of grouping in terms of indoors or outdoors, with or no glass and with or without usage of active illumination can enhance the recognition performance. Cross-scenarios are investigated using the texture based face matching schemes.

- Normalization of Data: Faces are canonicalized from the geometrical normalization method 


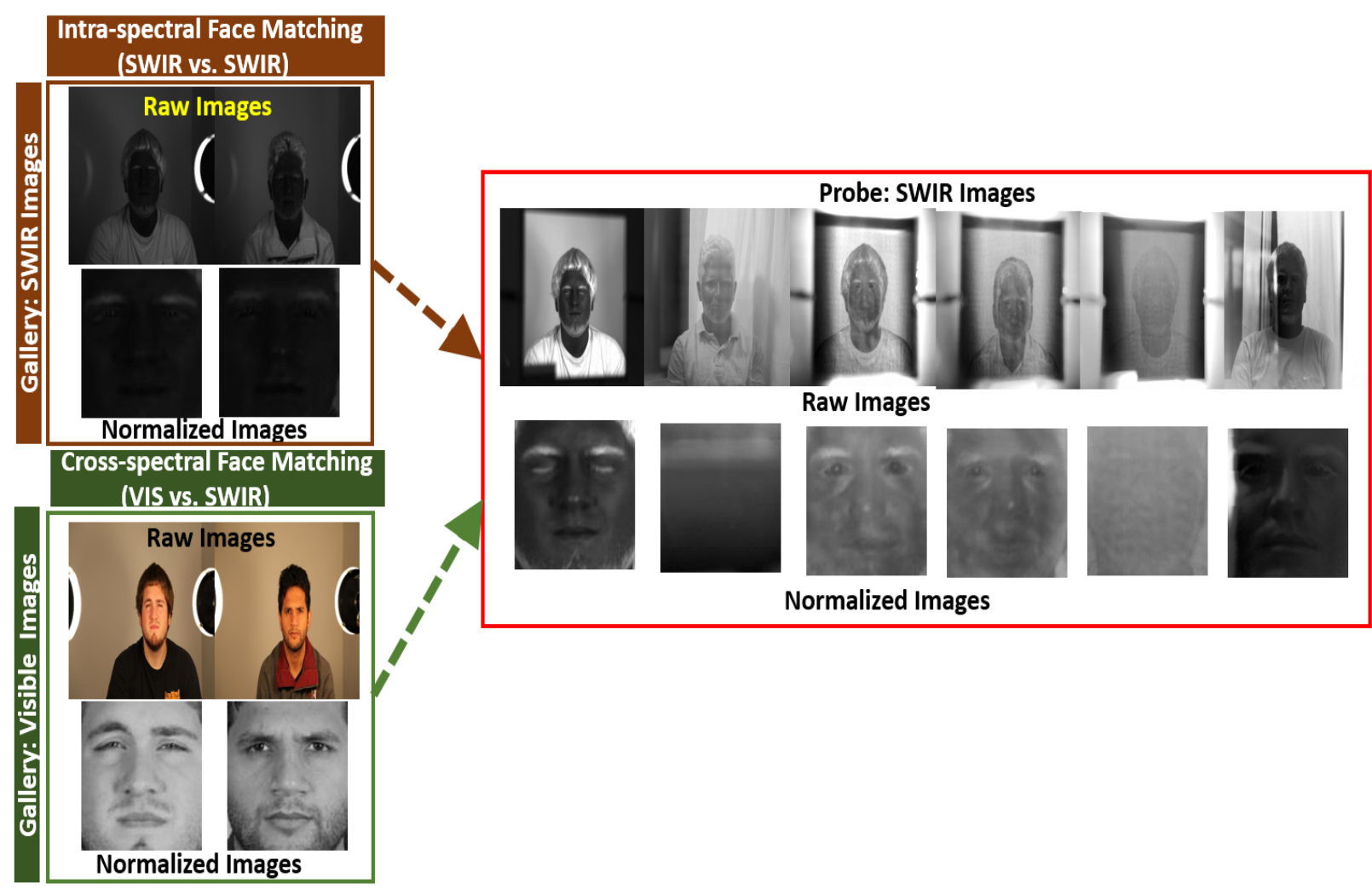

Figure 9.9: Face Matching Cross-Scenarios, Intra-spectral: Gallery images in the SWIR band matched against the Probe images in the SWIR band (Top). Cross-spectral: Gallery images in the Visible band matched against the Probe images in the SWIR band (bottom).

described in Chapter 4 to the same dimension of $128 \times 128$ pixels (see Fig. 9.9).

- Feature Extraction: To extract the features, LBP, Gabor and HOG descriptors are selected. The face images in our database contain unique characteristics and vary in sensor type (VIS and SWIR) as well as in the pose. To address this problem, kernel methods namely kernel linear discriminate analysis (KLDA) method is selected to extract the discriminant information from a high dimensional feature space.

The training data is used to find the non-linear directions and Fischer's linear discriminant by mapping into non-linear feature space. In the testing set, for each subject in the probe set (SWIR band), there is a corresponding gallery image (VIS band) as shown in Fig. 9.9. For each face image in the testing set (gallery and probe), first LBP, Gabor and HOG features are extracted and independently projected into the KLDA subspace (as discussed in Chapter 4 in equations 4.1 and 4.2). The distance scores are measured using the cosine distance score method (as illustrated in equation (4.3)) that achieved the best results in comparison to the L2 
normalization. As such, the distance scores for each selected feature extraction method are computed. The score normalization is performed using the $z$-score (score values lie between 0 and 1). Finally, the scores are fused based on the developed fusion scheme (Study 1) as presented in pseudo code algorithm 1 in Chapter 4.

\subsubsection{Baseline Face Matching Results}

Following two different sets of face matching experiments were selected:

Intra-Spectral Matching: In this experiment, the baseline images in SWIR band are compared to SWIR images captured indoors, outdoors (with and no glass and with and without the usage of active illumination respectively) as shown in Fig. 9.9. The results are compared with and without the usage of grouping. The identification results using both the academic and commercial face matchers are summarized in Table 9.2. Based on the results, it is determined that for the level 1 , the rank-1 identification accuracy improved from $48 \%$ to $62 \%$ for indoor class. The CMC curves for the first 5 ranks, for the best performed set out of randomly selected sets, are represented in Fig. 9.10(b).

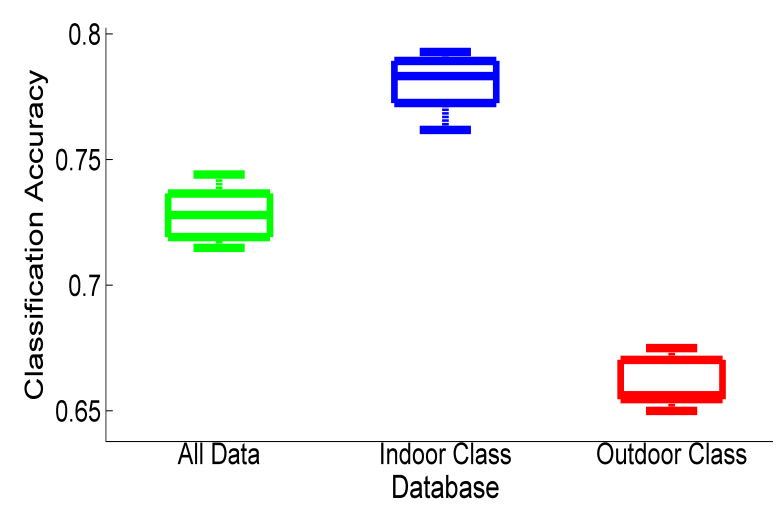

(a) All Data vs. Level 1 Grouping from Developed

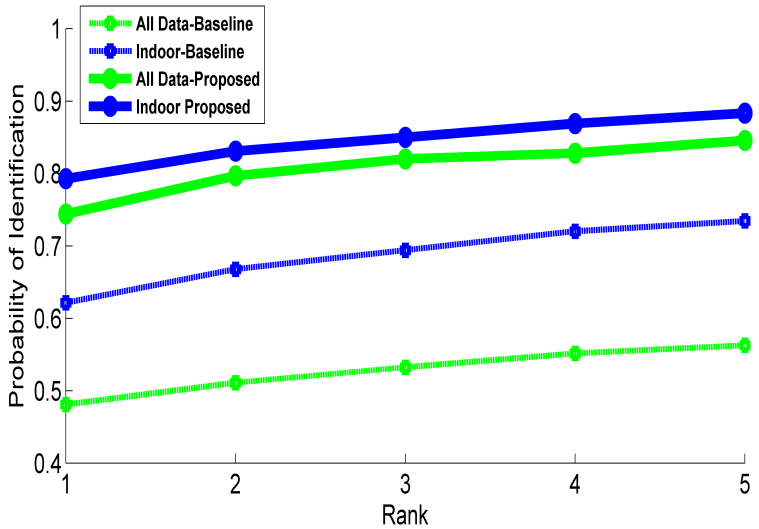

(b) Baseline vs. Developed

Figure 9.10: Intra-spectral face matching scenarios for SWIR (Gallery) against SWIR (Probe) for with and without grouping of the data into indoors or outdoors. Left: Box-plots for intraspectral face matching after running each experiment 5 times. Right: Each algorithm was run 5 times and here the rank-1 identification accuracy is presented from the best set.

Cross-Spectral Matching: The visible (controlled conditions) face images are compared to SWIR face images captured indoors, outdoors, with and without glass and active illumination 
Table 9.2: In this table the results for the intra-spectral, cross-spectral matching scenarios for with and without grouping of the data are presented. Intra-spectral matching scenario for SWIR (Gallery) against SWIR (Probe) under unconstrained conditions. Cross-spectral matching scenario for VIS (Gallery) against SWIR (Probe) under unconstrained conditions. NIR 60m (Probe)

\begin{tabular}{|c|c|c|c|c|c|}
\hline \multicolumn{7}{|c|}{ Intra-spectral: SWIR vs. SWIR } \\
\hline \hline Datasets & LBP-CHI & LTP-DT & LBP-DT & LTP-CHI & Developed \\
\hline All Data & 0.39 & 0.48 & 0.39 & 0.47 & 0.75 \\
Indoor & 0.44 & 0.62 & 0.43 & 0.60 & 0.80 \\
\hline \multicolumn{7}{|c|}{ Cross-Spectral: VIS vs. SWIR } \\
\hline \hline All Data & 0.07 & 0.20 & 0.08 & 0.20 & 0.67 \\
\hline \multicolumn{7}{|c|}{ Level 1: Indoor vs. Outdoor } \\
Indoor & 0.15 & 0.25 & 0.16 & 0.27 & 0.67 \\
Outdoor & 0.09 & 0.20 & 0.10 & 0.20 & 0.57 \\
\hline Level 2: With Glass (WTG) vs. Without Glass (WTOG) \\
Indoor WTOG & 0.26 & 0.46 & 0.28 & 0.46 & 0.96 \\
Indoor WTG & 0.13 & 0.22 & 0.14 & 0.23 & 0.63 \\
Outdoor WTOG & 0.10 & 0.25 & 0.11 & 0.25 & 0.77 \\
Outdoor WTG & 0.08 & 0.14 & 0.08 & 0.14 & 0.37 \\
\hline \multicolumn{7}{|c|}{ Level 3: With Active Illuminator (WTIL) vs. } \\
Indoor WTOIL & 0.21 & 0.34 & 0.22 & 0.36 & 0.89 \\
Indoor WTIL & 0.06 & 0.11 & 0.06 & 0.10 & 0.40 \\
Outdoor WTOIL & 0.16 & 0.32 & 0.16 & 0.33 & 0.78 \\
Outdoor WTIL & 0.03 & 0.07 & 0.03 & 0.07 & 0.78 \\
\hline
\end{tabular}

images. Cross-spectral face matching results are summarized in Table 9.2 for with and without the usage of grouping of the data.

Based on the results, it is determined that for the level 1 classification, the rank-1 identification accuracy improved from $20 \%$ to $27 \%$ for the indoor class. For the level 2 classification, the identification performance improved from $20 \%$ to $46 \%$ for the indoor class without glass as shown in Fig. 9.11(b), For the indoor class with glass, the identification performance improved from $20 \%$ to $23 \%$. For the level 3 classification, the identification improved from $20 \%$ to $36 \%$ for the indoor class without the usage of active illumination. For the outdoor class without the usage of active illumination, the identification improved from $20 \%$ to $33 \%$ as presented in Table 9.2

For all the levels of the classification, better performance results are achieved in comparison to the original FR system, namely when no grouping of the data is performed. The CMC curves 


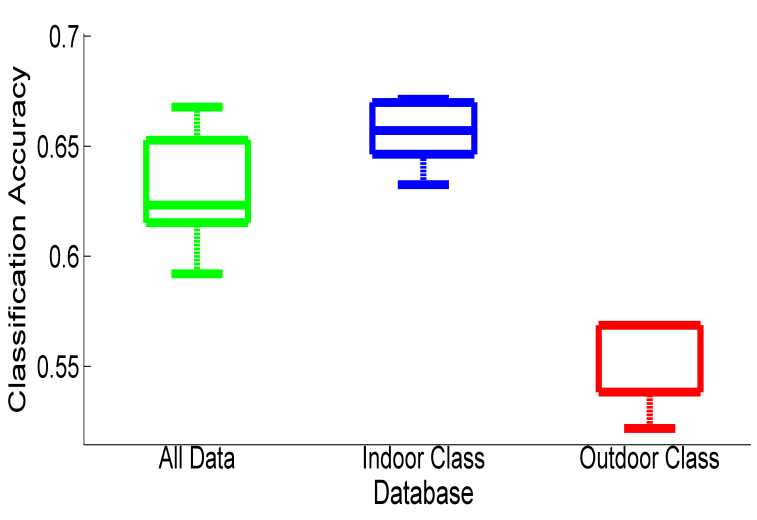

(a) All Data vs. Level 1 Grouping from Developed

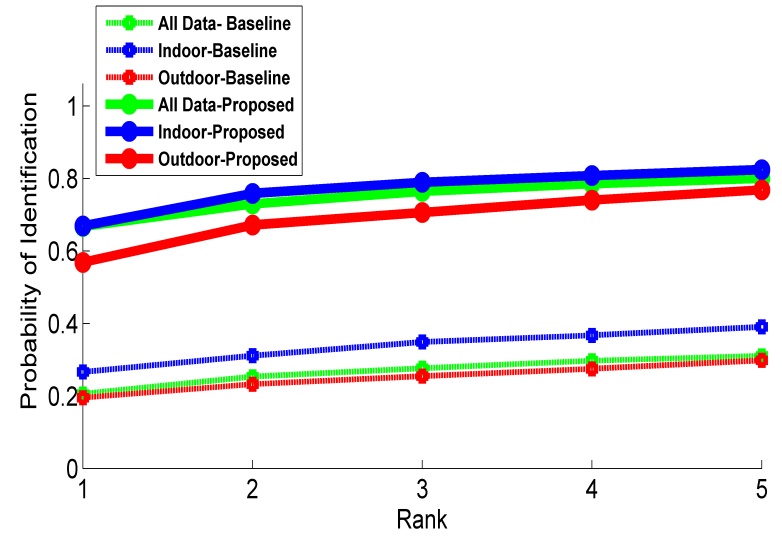

(b) Baseline vs. Developed

Figure 9.11: Cross-spectral face matching scenarios for VIS (Gallery) against SWIR (Probe) for with and without grouping of the data into indoors or outdoors. Left: Box-plots for crossspectral face matching after running each experiment 5 times. Right: The rank-1 identification accuracy is presented from the best set.

for the first 5 ranks, for the best performed set out of randomly selected sets, are presented in Figs. 9.12(b) (All Data vs. Indoor) and 9.12(c)(All Data vs. Outdoor).

\subsubsection{Face Matching Results using Developed method}

The experimental results indicate that for LBP/LTP based methods, the best overall accuracy reaches $62 \%$ (intra-spectral for indoor class) and $27 \%$ for cross-spectral with indoor class. These results are not satisfying. Thus, more complicated, but much more efficient approach is developed. First, DT and CHI distance transform methods are replaced by a kernel subspace method. A multi-feature score level fusion scheme is developed, where the fusion of the scores is performed based on the decision level scheme. In order to examine the effectiveness of FR algorithms, each experiment is repeated 5 times for each cross-scenario (i.e. each time a different training set was randomly selected) using the developed face recognition methods. The rank-1 identification accuracy is compared for the developed and baseline methods.

Intra-Spectral Matching: In this SWIR to SWIR face images are compared. 50\% of the data is used for the training (140 subjects and 7 samples per subject). The rest of the data (subjects) is used for testing (no overlap of subjects) i.e. 1 face image for each test subject in the SWIR band (Indoor class without glass) for gallery set and 6 face images for the SWIR probe set. Based on the results, it is determined that the developed approach achieved significant im- 
provement compared to baseline. For example, the rank-1 identification accuracy for All Data (without grouping) is $75 \%$ using the developed method, when compared to only $48 \%$ from the baseline. For the grouping of the data into indoor class, the rank-1 identification accuracy is $62 \%$. While from the developed method, the rank-1 accuracy is $80 \%$ as presented in Table 9.2 . The CMC curves for the first 5 ranks, for the best performed set are shown in Fig. 9.10(b), Cross-Spectral Matching: In this experiment, $50 \%$ of the data is used to train the system (140 subjects and 8 samples per subject - 1 face image in VIS and 7 face images in SWIR band for each subject). The rest of the data is used for testing i.e. 1 face image for each test subject in the visible band for gallery set and 7 face images in the SWIR for probe set (challenging database collected indoors, outdoors, with and without glass, with and without active illuminator). There is no subject overlap between the training and test sets.

All Data vs. Level 1: Based on the results, it is determined that the developed method achieved better performance results compared to the baseline systems. For all data without any grouping, the identification rank-1 accuracy is $67 \%$ from the developed method and $20 \%$ from the baseline (LTP-DT). The significant improvement is achieved from the developed method over the academic methods for grouping of the data into indoor and outdoor class. For indoor class, the identification accuracy is $67 \%$ for the developed and $27 \%$ from the baseline. For outdoor class, the identification accuracy is $57 \%$ for the developed, and $20 \%$ from the baseline as presented in Table 9.2. The CMC curves for the first 5 ranks, for the best performed set are shown in Fig. 9.11(b),

All Data vs. Level 2: Significant improvement is achieved from the developed method over the academic methods for further grouping of the indoor and outdoor class into with and without glass. For indoor class into indoor without tinted glass (WTOG), the identification rank-1 accuracy is $96 \%$ for the developed and $46 \%$ from the baseline as shown in Fig. 9.12(b), For indoor class into with glass (WTG), the rank-1 identification accuracy is $63 \%$ for the developed method and 23\% (LTI-CHI) from the baseline. For outdoor class into without glass, the identification accuracy is $77 \%$ for the developed, and $25 \%$ from the baseline as presented in Table 9.2. For the outdoor class into with glass, the rank-1 identification accuracy improved from 14\% (baseline) to 37\% from the developed system. The CMC curves for the first 5 ranks, for 


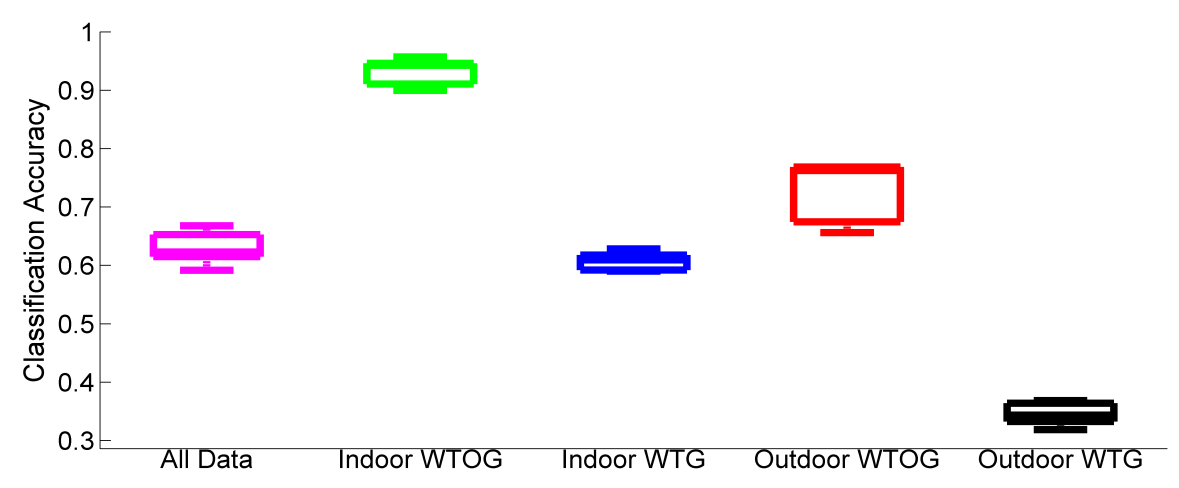

(a) All Data vs. Level 2 Grouping from Developed

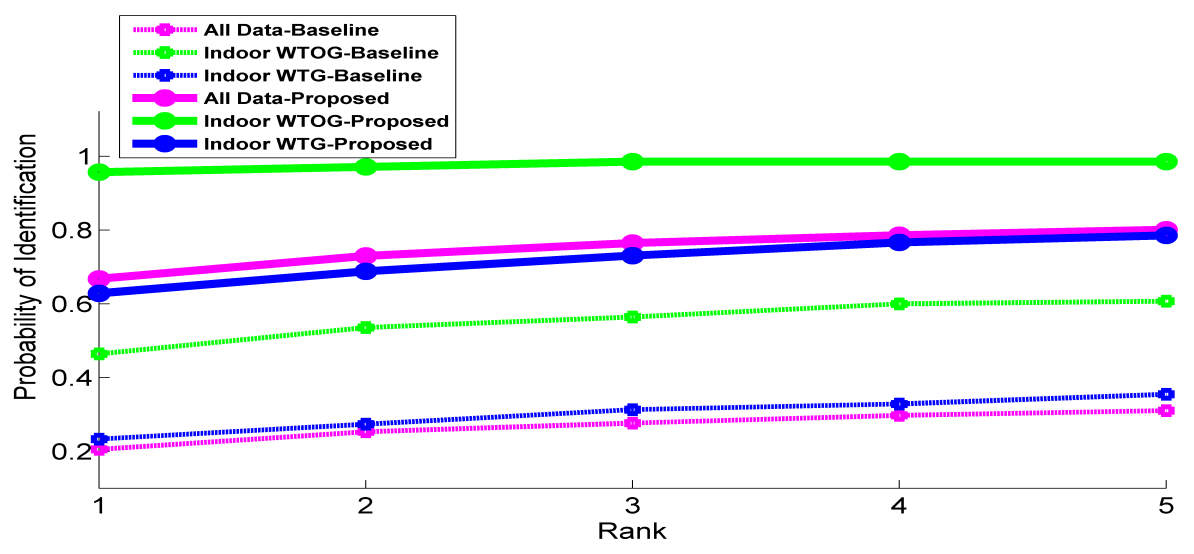

(b) Baseline vs. Developed (Indoor)

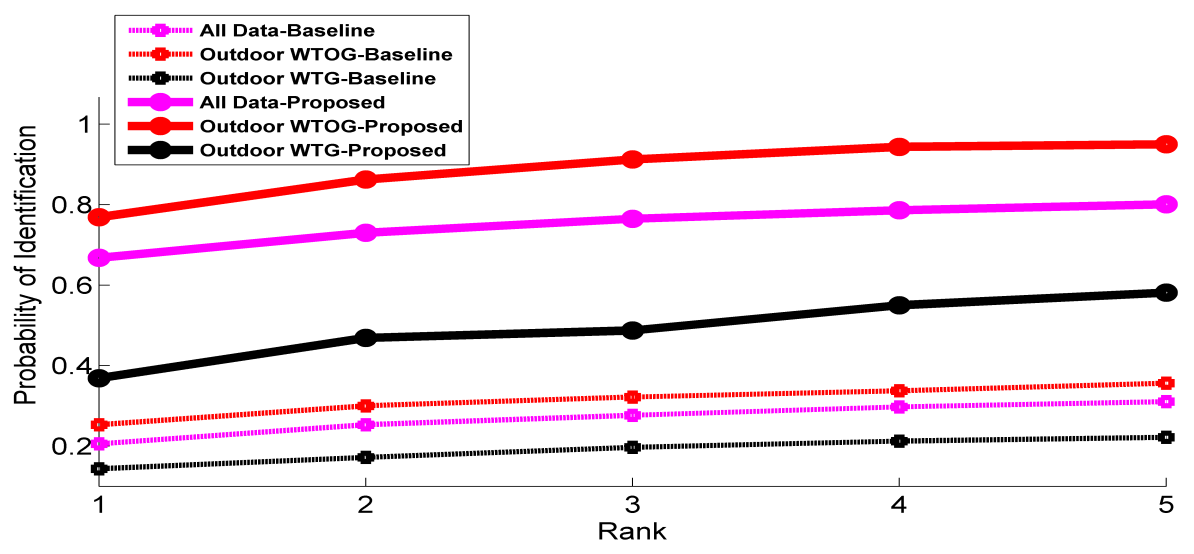

(c) Baseline vs. Developed (Outdoor)

Figure 9.12: Cross-spectral (VIS vs. SWIR) face matching results for with and without grouping of the data into indoors and outdoors class into with and without glass. Top: Box-plots for cross-spectral face matching after running each experiment 5 times. Bottom-Left: Each algorithm was run 5 times and here the rank-1 identification accuracy is presented from the best set for All Data (Without Grouping) and Indoor class into Indoor Without Glass (IndoorWTOG) or Indoor With Glass (Indoor-WTG). Bottom-Right: For All Data and Outdoor class into Outdoor Without Glass (Outdoor-WTOG) or Outdoor With Glass (Indoor-WTG). 


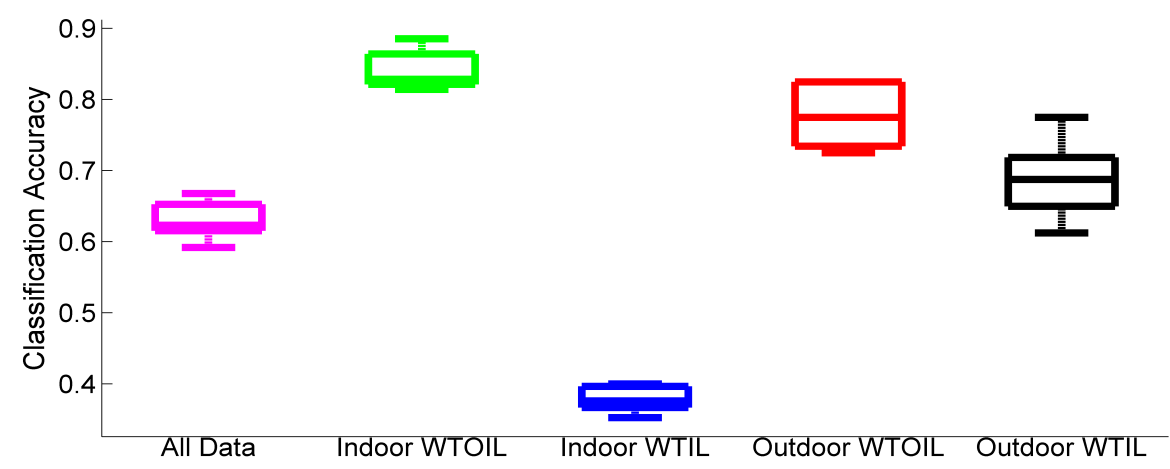

(a) All Data vs. Level 3 Grouping from Developed

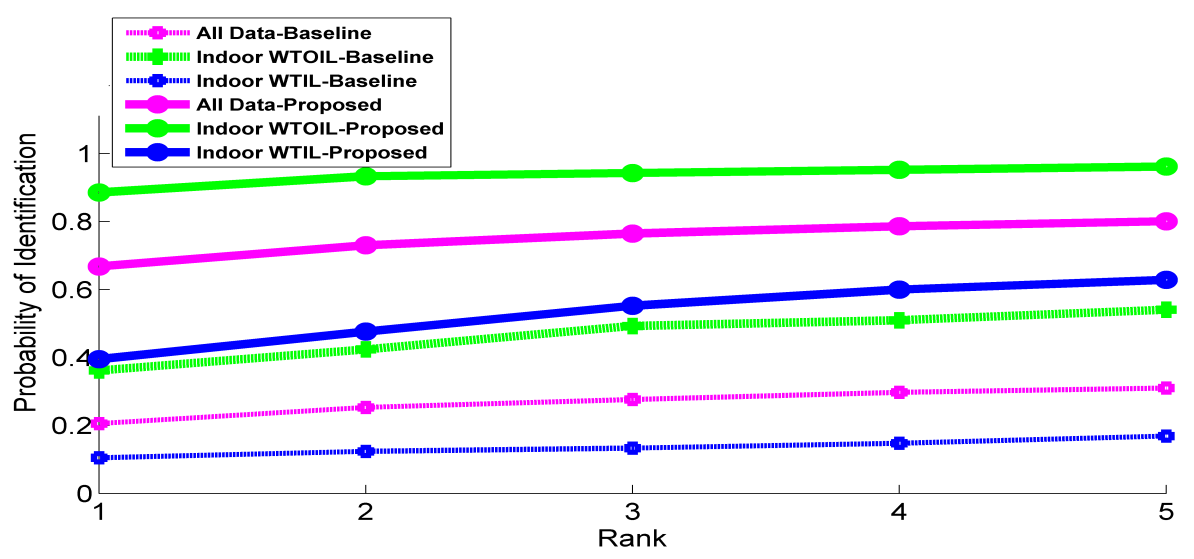

(b) Baseline vs. Developed (Indoor)

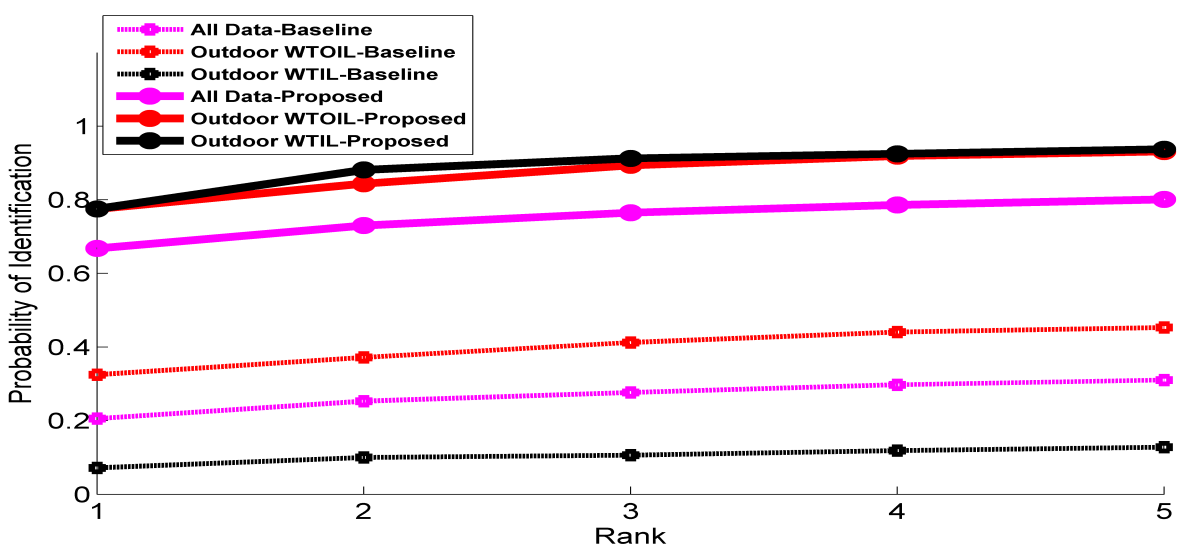

(c) Baseline vs. Developed (Outdoor)

Figure 9.13: Cross-spectral (VIS vs. SWIR) face matching results for with and without grouping of the data into indoors and outdoors class into with and without active illuminator. Top: Box-plots for cross-spectral face matching after running each experiment 5 times. BottomLeft: Each algorithm was run 5 times and here the rank-1 identification accuracy is presented from the best set for All Data (Without Grouping) and Indoor class into Indoor Without Illuminator (Indoor-WTOIL) or Indoor With Illuminator (Indoor-WTIL). Bottom-Right: For All Data and Outdoor class into Outdoor Without Illuminator (Outdoor-WTOIL) or Outdoor With Illuminator (Outdoor-WTIL). 
the best performed set are shown in Figs. 9.12(b) and 9.12(c).

Based on the results, it is determined that, after grouping of the data into Level 2 (indoor and outdoor class into WTOG and WTG), the identification rank-1 accuracy improved from $67 \%$ (All Data without grouping) to $95 \%$ for Indoor WTOG, and 77\% for the outdoor WTOG. For the Indoor WTG, results are similar and the performance is low for the outdoor WTG. There is a valid reason for the poor performance, for example the factors like the quality of probe image degrades due to tinted glass and outdoor conditions.

All Data vs. Level 3: Based on the results, it is concluded that using the developed method in comparison to the baseline method, the identification accuracy improved from $36 \%$ (baseline) to $89 \%$ (developed) for Indoor WTOIL, from $11 \%$ to $40 \%$ for Indoor WTIL, from $33 \%$ to $78 \%$ for Outdoor WTOIL and from $7 \%$ to $78 \%$ for Outdoor WTIL as presented in Table 9.2. The CMC curves for the first 5 ranks, for the best performed set are shown in Figs. 9.13(b) and 9.13(c),

Based on the results, it is determined that, after grouping of the data into Level 3 (indoor and outdoor class into WTOIL and WTIL), the identification rank-1 accuracy improved from $67 \%$ (All Data without grouping) to $89 \%$ for Indoor WTOIL, and $78 \%$ for the outdoor WTOIL and WTIL.

\subsection{Classification and Face Matching for SSMW Database}

To conduct this study, multi-wavelength data was captured at five different SWIR wavelengths ranging from $1150 \mathrm{~nm}$ to $1550 \mathrm{~nm}$ in the increments of $100 \mathrm{~nm}$ as described in Chapter 3. In [3], classification scheme was developed using the traditional machine learning models. An automated quality-based score level fusion scheme was developed for the classification of input multi-wavelength images. The image quality factors such as sharpness, blurriness, structural content and contrast are selected as features. These features (quality scores) were measured using reference and no-reference based quality assessment methods. The classification was performed using Bayesian and $k N N$ models as shown in Fig. 9.14. The classification accuracy was greater than $96 \%$. The key problem was the selection of features for the fusion, 
(a)

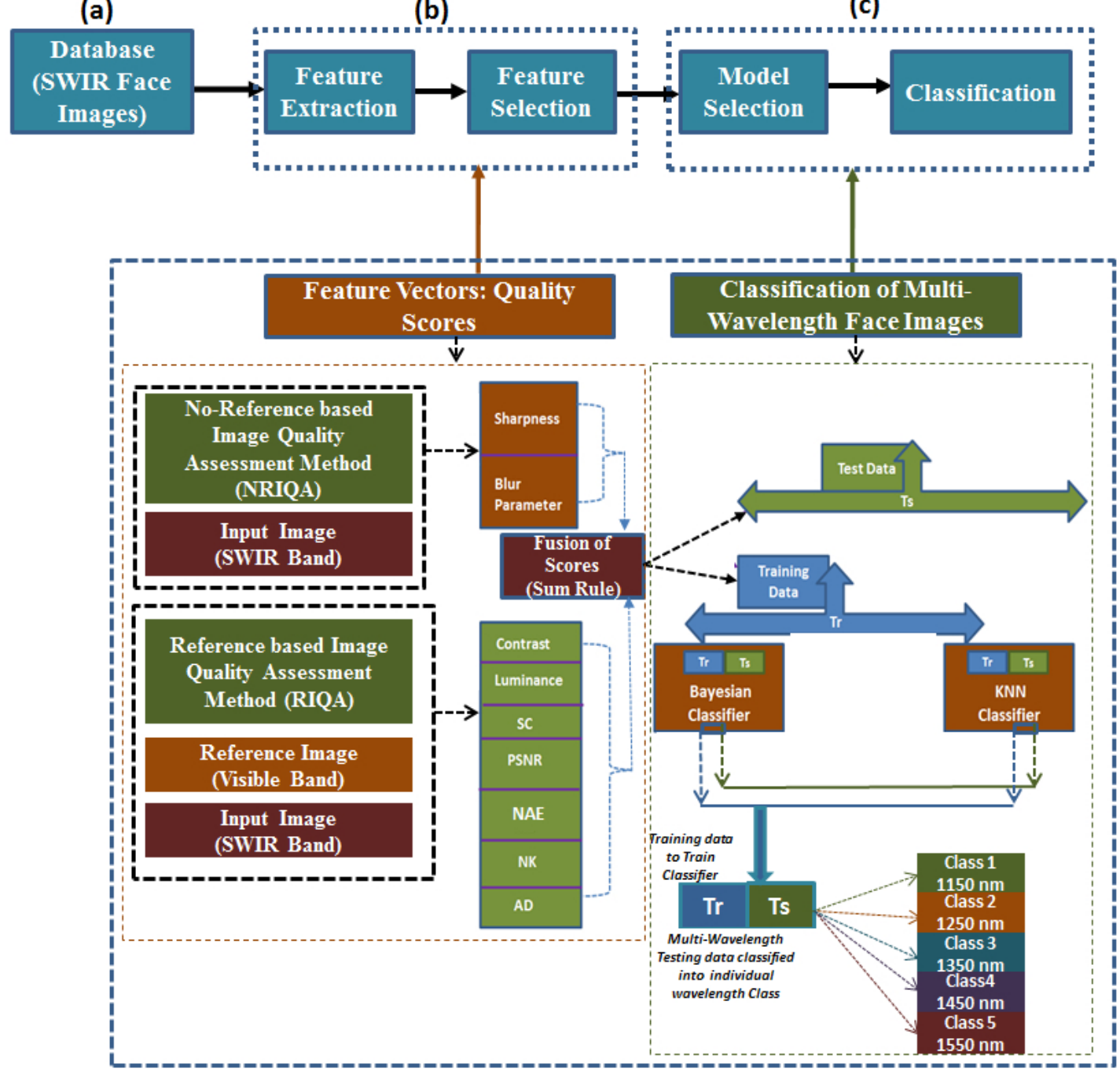

Figure 9.14: Overview of the developed fusion scheme that performs wavelength-based classification. (b) Feature extraction is performed based on methods presented in the bottom figure at left side and (c) classification is performed based on methods presented in bottom figure at right side [3].

as the computed image quality scores were close to each other [3, 74]. The classification was performed based on individual subjects. The main steps for the classification approach were; feature extraction, feature selection, model selection and evaluation of the classifier.

To address these issues, $\mathrm{CNN}$ based classification model was developed, where the selection of features is performed automatically. The classification is performed based on all data (all subjects). The classification results are compared from developed CNN network for individual subjects and all data.

The face data is categorized into individual wavelengths from the developed CNN based situation classification framework, before the FR algorithms are used. There is no pre-trained data available for the challenging SWIR datasets. To deal with this issue, the models are trained 
on our database. The model parameters (e.g. epoch and momentum) are empirically optimized and are used to perform the classification more accurately. After situation classification, a set of face matching experiments are performed where a developed face matching fusion approach is used, indicating that when fusion is supported by the classification framework, the rank-1 identification rate is significantly improved compared to when no classification is used whatsoever.

\subsubsection{Classification using Developed CNN Architecture}

To perform the classification, two databases collected in the lab namely WVU-SSMW and WVU Multi Scenario (MS) are selected. WVU-MS database consists of 140 subjects. The database consists of face images at five different wavelengths of 1150, 1250, 1350, 1450 and $1550 \mathrm{~nm}$. WVU-SSMW database consists of face images from 30 subjects at 1150, 1250, 1350, 1450 and $1550 \mathrm{~nm}$.

- Model Architecture: First images are rescaled to $32 \times 32$ and then applied to the CNN

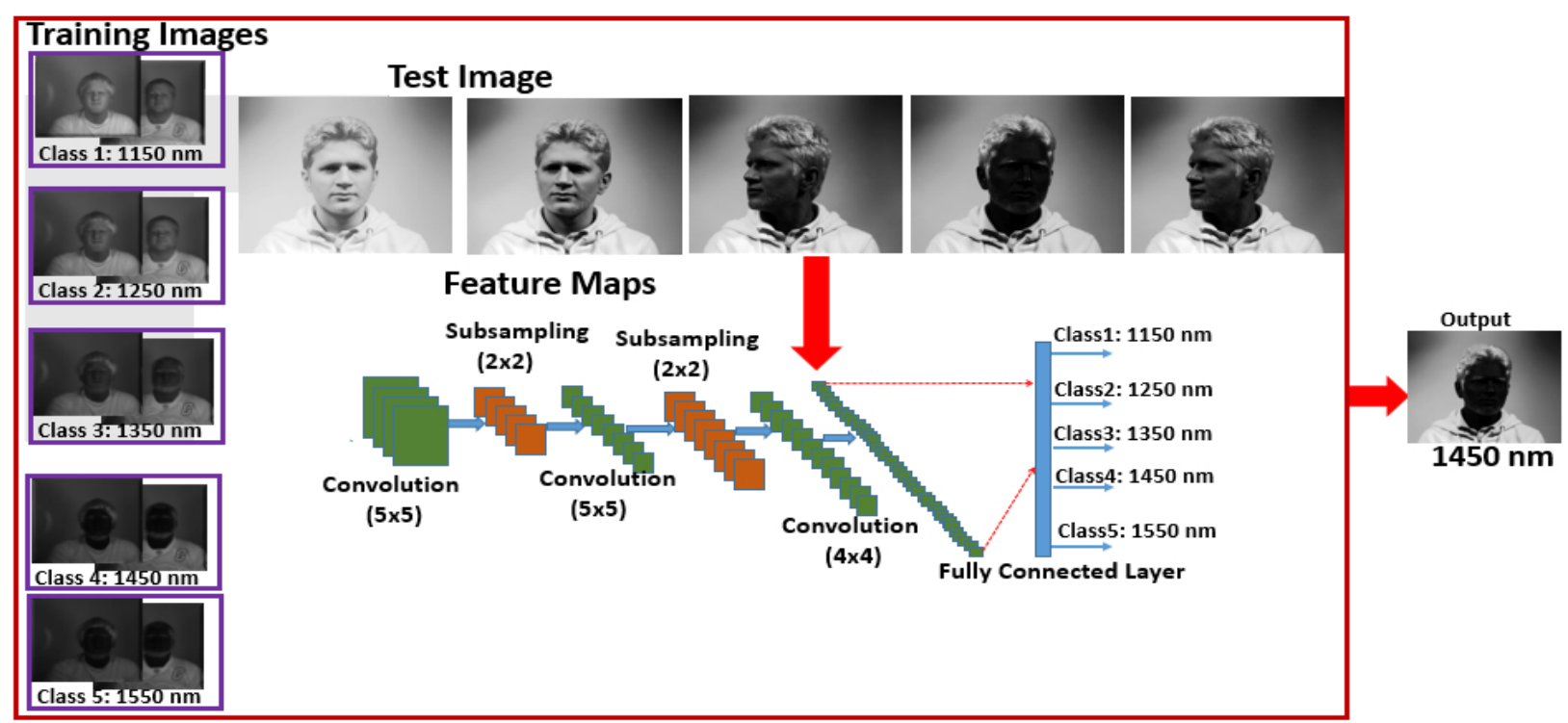

Figure 9.15: An overview of the developed CNN architecture.

network using the same architecture as discussed in Chapter 4. The output of this layer is fed to a soft-max layer that assigns a label to each class, i.e. 1150 or 1250 or 1350 or 1450 or 1550 nm for WVU-SSMW database as shown in Fig. 9.15 . 

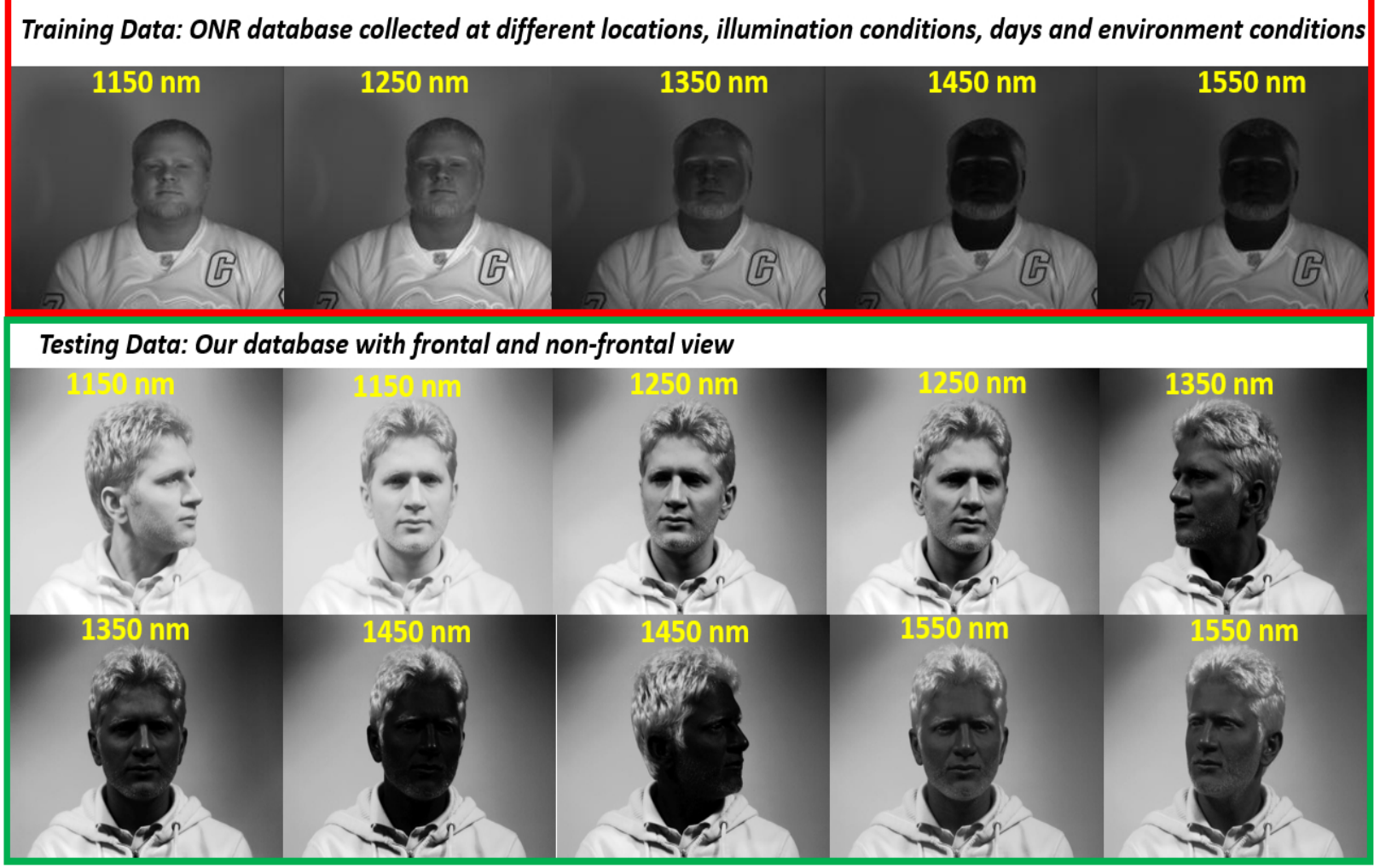

Figure 9.16: Training and Testing Data for CNN Architecture for Classification.

-Training and Testing Data: In the experiments performed, the subjects in the training and test sets are different, and the images are taken at different locations and days as shown in Fig. 9.16. Two scenarios are selected for the classification based on individual subjects while for the classification based on all data (all subjects) three scenarios are selected.

The classification is performed for two sets including:

- Set 1, is based on individual subjects and two scenarios are selected:

- Scenario 1, is based on the training using WVU-MS database $(1150,1250,1350,1450$ and $1550 \mathrm{~nm}$ ground truth images collected indoors in SWIR band). For the testing, WVU-SSMW database (individual subjects) is selected.

- Scenario 2, where the training data set is extended using WVU-SSMW database. The SWIR images $(1150,1250, \ldots, 1550 \mathrm{~nm})$ from WVU-MS and WVU-SSMW (20\% of data) are selected to train the CNN network and rest of the WVU-SSMW database is selected for the testing.

- Set 2, is based on all the data and three scenarios are selected: 
- Scenario 1, where, WVU-MS (DB1) face images (with frontal view) are used for the training and WVU-SSMW (DB2) database (with frontal and non-frontal view) is used for testing.

- Scenario 2, the WVU-MS database [61] is selected and the training data set is extended by using the WVU-SSMW database (only $20 \%$ subjects). The rest of the data from WVU-SSMW database is selected for testing (no overlap of subjects).

- Scenario 3, the WVU-MS database is selected and the training data set is extended by using the WVU-SSMW database (80\% subjects). The rest of the data from WVU-SSMW database is selected for testing (no overlap of subjects).

For Set 2, the momentum value of 0.92 and batch size of 100 are selected to train the CNN network. An empirical optimization on epoch parameter is conducted that resulted in better classification accuracy. A series of experiments are performed with the selected epoch values of $4,8, \ldots, 52$. This process is repeated five times for each epoch value using random selection of training and testing data (without overlap of subjects). For scenario 2 (set 2) using all the database (all subjects), based on mean and variance plots, the best classification results are achieved for an epoch value of 44 as shown in Fig. 9.17 and the classification accuracy is more than $80 \%$.

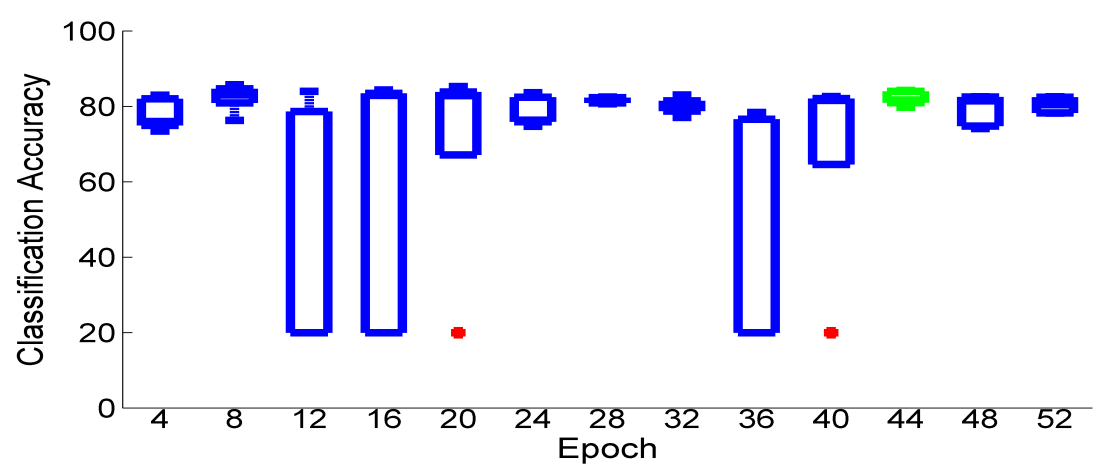

Figure 9.17: Classification accuracy results with a selected set of epoch for CNN. Each boxplot is based on results from 5 randomly selected training and testing sets.

For Set 2 based on all data, the classification accuracy is almost $35 \%$ for Scenario 1 (WVUMS for training). For Scenario 2 (with extended training using WVU-SSMW database), the 
classification accuracy is more than $80 \%$ and more than $95 \%$ for Scenario 3. In order to examine the effectiveness of classification framework, each experiment is repeated 4 times for each cross-scenario (i.e. each time a different training set was randomly selected) using the developed CNN network. The classification results after repeating each experiment 4 times are shown in Fig. 9.18

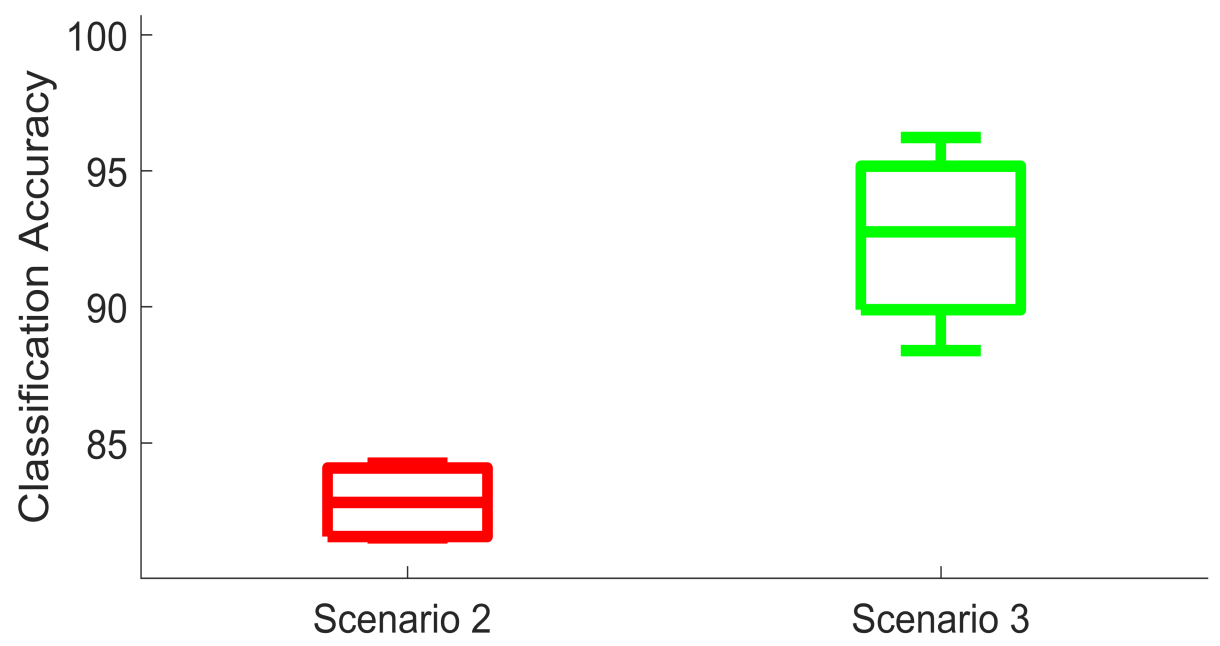

Figure 9.18: Classification accuracy results for Set 2: Scenario 2 and Scenario 3 with selected set of training sets for CNN. Box-plots are based on results from 4 randomly selected training and testing sets.

For Set 1 based on individual subjects the classification accuracy is almost $40 \%$ for Scenario 1 using the WVU-MS for training. For Scenario 2 with extended training set using WVUSSMW database, the classification accuracy is more than $90 \%$ as presented in Table 9.3 .

Table 9.3: Classification results based on developed CNN architecture.

\begin{tabular}{|c|c|}
\hline \multicolumn{2}{|c|}{ Classification Accuracy } \\
\hline Set 1 Database: Based on Individual Subjects \\
\hline Scenario 1 & 0.40 \\
\hline Scenario 2 & 0.94 \\
\hline \multicolumn{2}{|c|}{ Set 2 Database: Based on All Data } \\
\hline Scenario 1 & 0.36 \\
\hline Scenario 2 & 0.84 \\
\hline Scenario 3 & 0.96 \\
\hline
\end{tabular}

The promising classification performance results are achieved for the extended training database. The decrease in the performance of the system for scenario 1 using only WVU-MS 
database for the training is due to the variation in light conditions and sensors, under which the training images were collected. Moreover, the database consists of face images with only frontal view. Whereas, for the WVU-SSMW database (testing data) face images collected are left profile, right profile, and full frontal. To address this problem, the training data is extended in scenarios 2 and 3.

\subsubsection{Face Matching for SSMW Database}

In cross-scenario FR studies, the system performance is evaluated using academic and the developed face matchers based on score level fusion scheme. Cross-spectral (VIS vs. SWIR) face matching experiments are conducted. The main stages of face recognition system include, face normalization (neutral faces) and then identification, followed by feature extraction and matching.

\subsubsection{Frontal and Non-Frontal View Face Classification}

WVU-MS database consists of face images with frontal view. While, for WVU-SSMW database, the face images consist of both the full frontal (FF) and non-frontal (NFF) view. The classification of the face images into frontal or non-frontal is performed based on the developed weighted quality-based score level fusion scheme in [3]. The quality scores used for the classification of FF vs. NFF face images are computed based on reference and no-reference based quality assessment methods (such as the luminance, contrast, sharpness, blurriness). The five steps of the developed approach are presented in Fig. 9.19.

\subsubsection{Normalization of Data}

Eye locations were located automatically using algorithm developed in [98] and located eye coordinates were further used to normalize the face images to the same dimension of $128 \times 128$ pixels. 


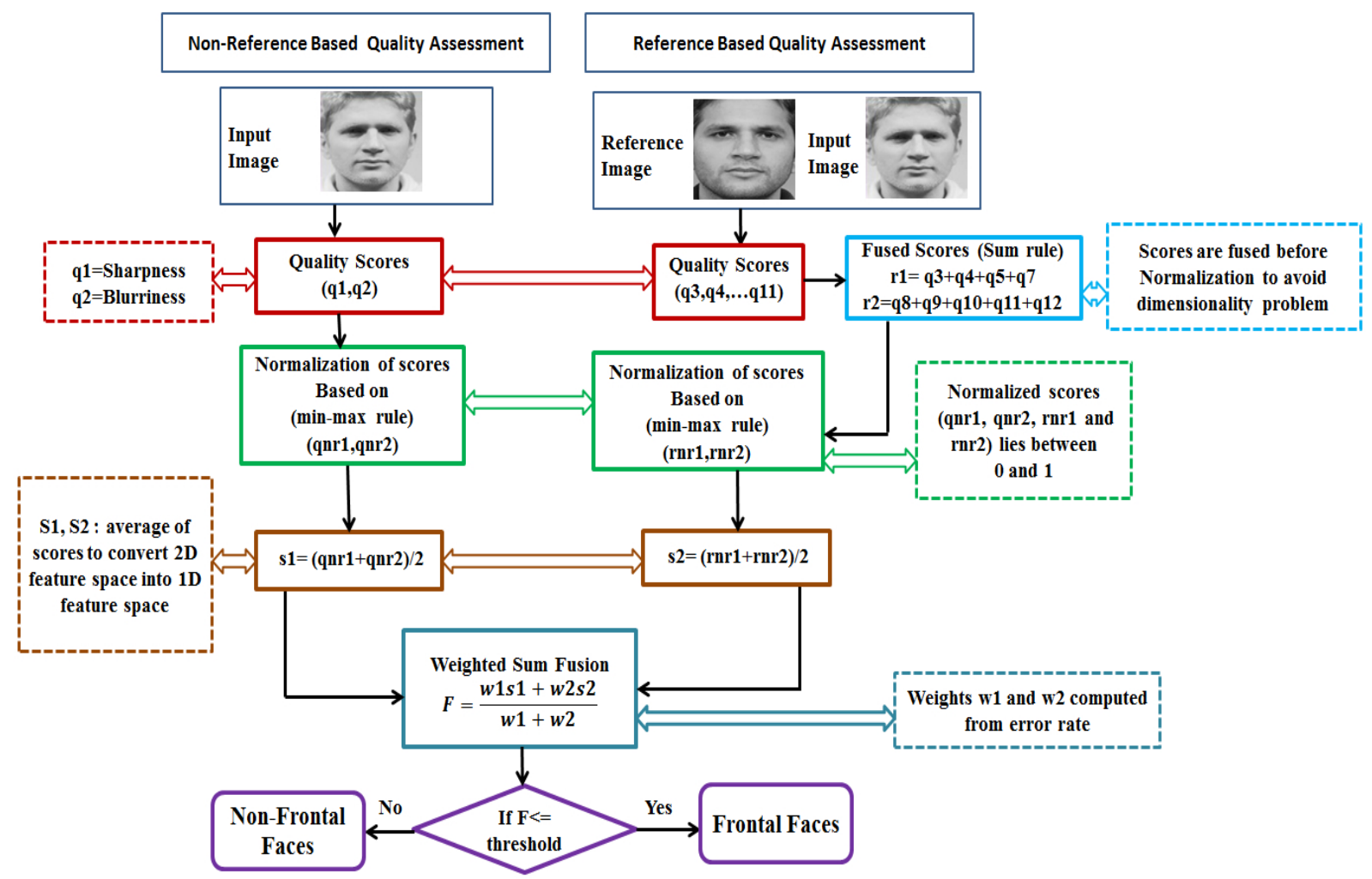

Figure 9.19: Flow chart diagram representing the process involved in the developed approach for classifying the frontal and non-frontal face images [3].

\subsubsection{Cross-Spectral Face Matching based on developed MFSDF Scheme}

The developed MFSDF based face matching scheme is applied to the challenging multiwavelength database where the face images are collected in the SWIR band with variation in pose angles as discussed in Chapter 3. The face images in the database contain unique characteristics and vary in sensor type (VIS and SWIR) as well as in the pose. To address this problem, KLDA method is selected to extract the discriminant information from a high dimensional feature space.

Two sets of face recognition experiments are performed using the developed MFSDF based face matcher. First, experiments are performed with the original FR system, namely when no grouping of the database $(1150,1250,1350,1450$ and $1550 \mathrm{~nm})$ is used. Without grouping, all data is used to perform the face matching experiments (VIS vs. All Data or Wavelengths). Second, experiments are performed to determine whether the usage of grouping in terms of individual wavelengths can enhance the recognition performance. Cross-scenarios are inves- 
Gallery: VIS

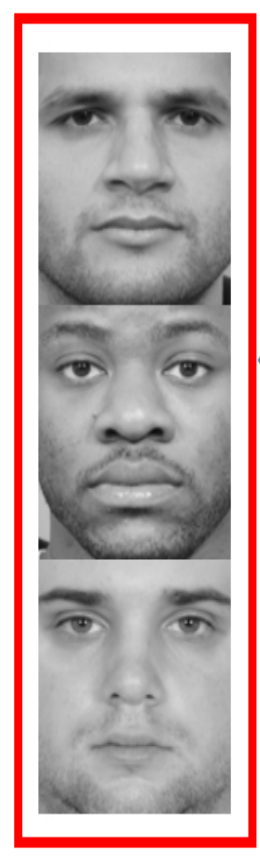

Probe: Face Images collected from SSMW Imaging System in SWIR

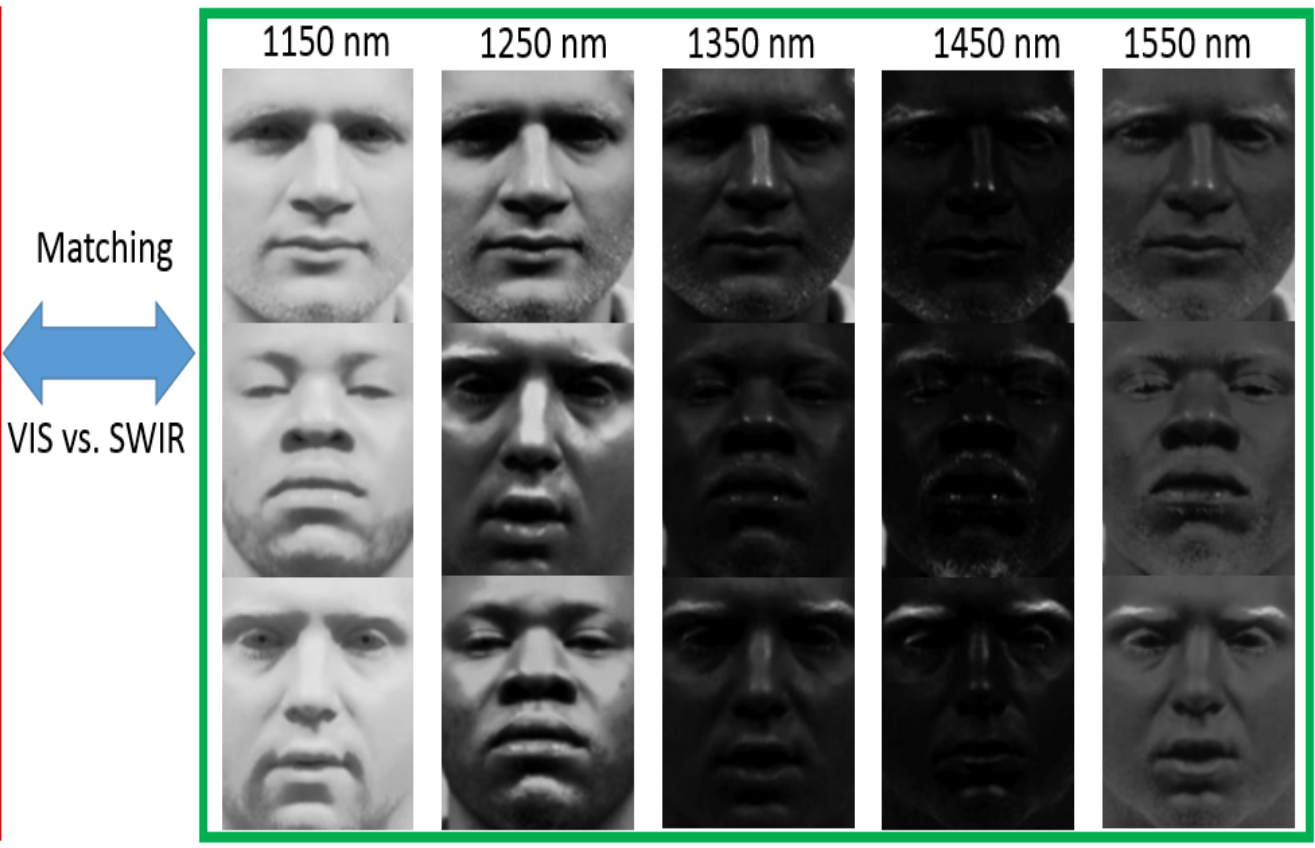

Figure 9.20: Cross-Spectral Face Matching: Gallery images in the VIS band (Left) are matched against the Probe images in the SWIR band captured from the designed SSMW system at five wavelengths of 1150, 1250, 1350, 1450 and $1550 \mathrm{~nm}$ (Right).

tigated using the developed face matching scheme. The classified database with labels; 1150 $\mathrm{nm}, 1250 \mathrm{~nm}, 1350 \mathrm{~nm}, 1450 \mathrm{~nm}, 1450 \mathrm{~nm}, 1550 \mathrm{~nm}$ from the developed deep learning system is used for face matching. For each dataset, the $50 \%$ data is randomly used for training set and rest of the data is used as the testing set, with no subject overlap. This process is repeated five times, using random selection of the training and test sets each time. For cross-scenarios, VIS vs. SWIR face matching is performed for 5 sets: VIS vs. $1150 \mathrm{~nm}$, VIS vs. $1250 \mathrm{~nm}$, VIS vs. $1350 \mathrm{~nm}$, VIS vs. $1450 \mathrm{~nm}$ and VIS vs. $1550 \mathrm{~nm}$ as shown in Fig. 9.21 .

Based on the results, it is determined that for grouping of data into individual wavelengths, the rank-1 identification accuracy improved from $66 \%$ to $87 \%$ for $1550 \mathrm{~nm}$ and from $66 \%$ to $80 \%$ for 1150 and $1350 \mathrm{~nm}$ wavelengths as shown in Fig. 9.21. For class with label of 1250 $\mathrm{nm}$ wavelength, the rank-1 identification accuracy improved from $66 \%$ to $70 \%$ but the rank-1 identification accuracy results are similar when the class with label of $1450 \mathrm{~nm}$ is selected. The poor performance is attributed to the atmospheric absorption effect for this wavelength. The spectral response (amount of absorbed and reflected light) is unique for each wavelength. 


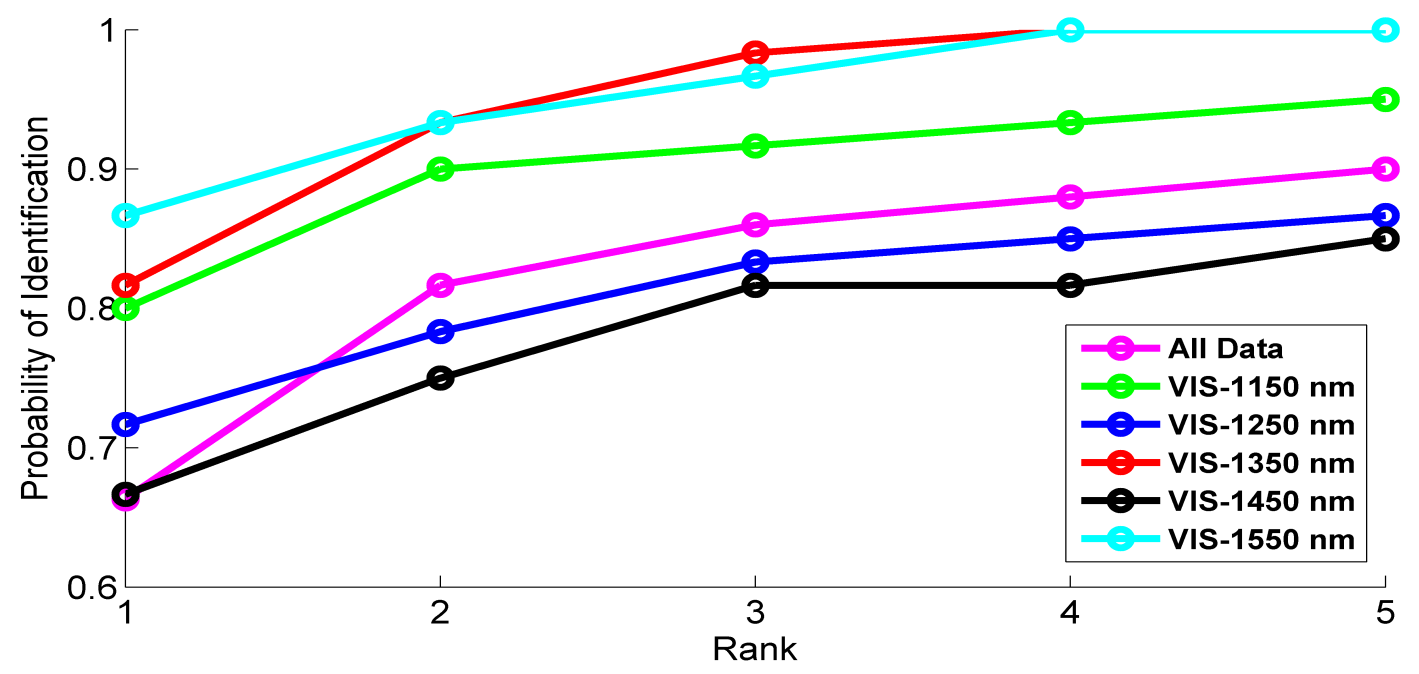

Figure 9.21: Cross-spectral face matching scenarios for VIS (Gallery) against SWIR (Probe) for with and without grouping of the data into individual wavelengths. The rank-1 identification accuracy is presented from the best set.

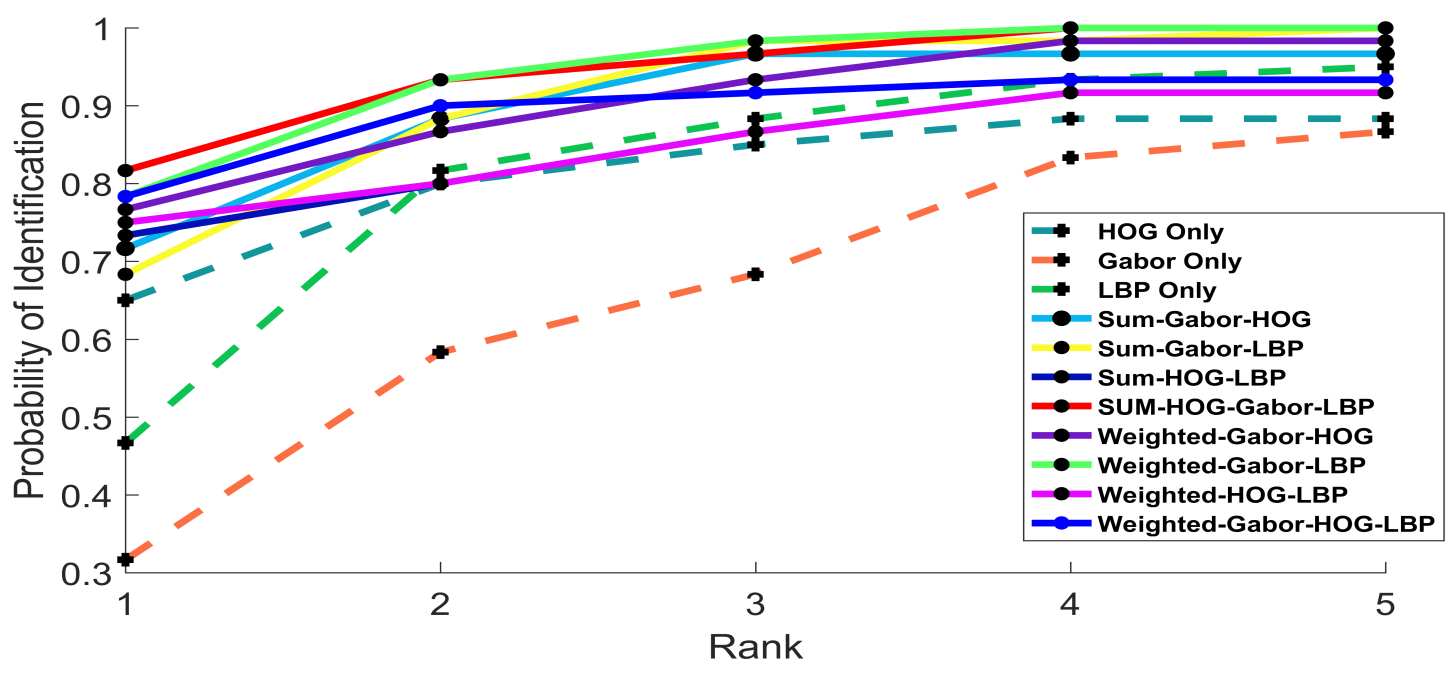

Figure 9.22: Cross-spectral face matching scenarios for VIS (Gallery) against SWIR $1350 \mathrm{~nm}$ (Probe) for the data into individual wavelengths. Comparison for individual vs. Fusion based matchers.

The present moisture content in the environment has an impact on the face appearance. Water vapors absorb more light energy absorption at $1450 \mathrm{~nm}$ and hence images appear dark for this wavelength as shown in Fig. 9.20. A comparison between the individual descriptor and based on the fusion schemes is presented in Fig. 9.22 for the probe images labeled with $1350 \mathrm{~nm}$ wavelength and Fig. 9.24 for probe images labeled as $1550 \mathrm{~nm}$. 


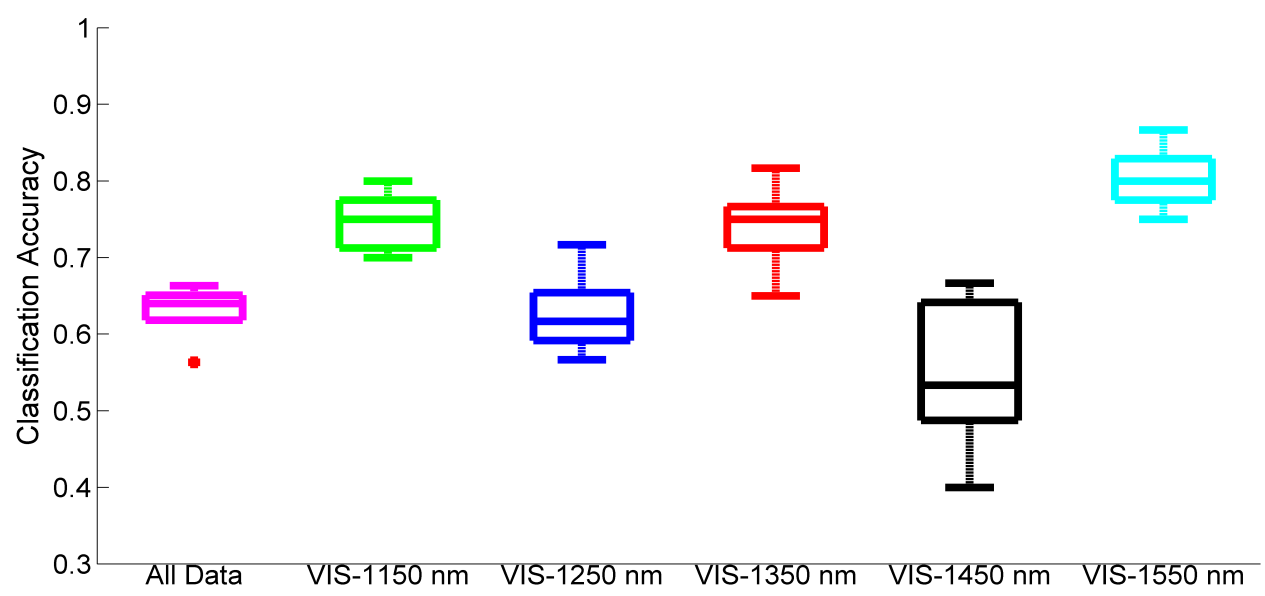

Figure 9.23: Cross-spectral face matching scenarios for VIS (Gallery) against SWIR (Probe) for with and without grouping of the data into individual wavelengths. Box-plots for crossspectral face matching after running each experiment 5 times.

Table 9.4: The Cross-spectral matching scenarios for VIS 1.5m (Gallery) against SWIR (1150, $1250,1350,1450$ and $1550 \mathrm{~nm}$ ) are performed. The experimental results are presented when the developed FR algorithms is run using 50\% of the data for training and the rest of the data for testing (with no subject overlap). The experiments were run 5 times and the rank-1 identification accuracy is presented here are as average.

\begin{tabular}{|c|c|c|c|c|c|}
\hline \multicolumn{7}{|c|}{ Gallery VIS vs. SWIR } \\
\hline Rank & Rank 1 & Rank 2 & Rank 3 & Rank 4 & Rank 5 \\
\hline All Data & 0.63 & 0.80 & 0.88 & 0.91 & 0.94 \\
$1150 \mathrm{~nm}$ & 0.74 & 0.83 & 0.87 & 0.90 & 0.91 \\
$1250 \mathrm{~nm}$ & 0.62 & 0.77 & 0.85 & 0.88 & 0.91 \\
$1350 \mathrm{~nm}$ & 0.74 & 0.91 & 0.97 & 1.00 & 1.00 \\
$1450 \mathrm{~nm}$ & 0.52 & 0.72 & 0.81 & 0.83 & 0.91 \\
$1550 \mathrm{~nm}$ & 0.80 & 0.88 & 0.93 & 0.96 & 0.97 \\
\hline
\end{tabular}

\subsection{Contribution}

\subsubsection{Evaluation for Multi-Scenario Database}

- A facial imaging system is developed in the SWIR band with an aim of improving the performance of face recognition system.

- The experimental results for the data filtering showed that for Level 1 classification, CNN 


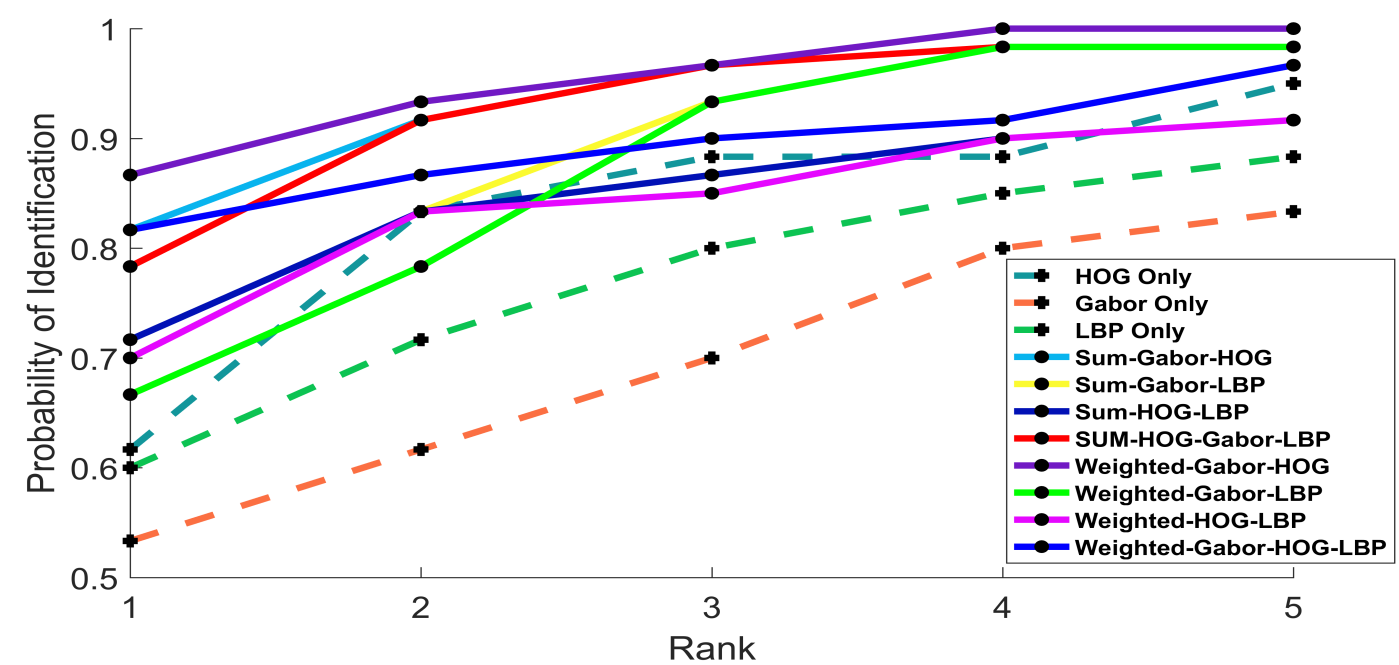

Figure 9.24: Cross-spectral face matching scenarios for VIS (Gallery) against SWIR $1550 \mathrm{~nm}$ (Probe) for the data grouped into individual wavelengths. Comparison of individual vs. Fusion based matchers.

provided with significantly improved classification accuracy, more than $95 \%$ in most of the tested scenarios. For example, the classification accuracy is more than $98 \%$ for level 2, and is more than $95 \%$ for level 3 classification.

- A series of face identification experiments are performed for intra-spectral and crossspectral scenarios. The results provide important evidence that the data filtering provide significant improvement in the rank-1 identification rate for both intra-spectral and crossspectral scenarios. For intra-spectral, the rank-1 identification accuracy improved from $75 \%$ to $80 \%$. For cross-spectral face matching, there is a significant improvement in the rank-1 identification accuracy from $67 \%$ to $96 \%$ for Level 2 classification for the indoor class without glass and $67 \%$ to $77 \%$ for the outdoor class without glass. The rank-1 identification accuracy reaches more than $85 \%$ for the indoor class without illuminator and more than $75 \%$ for outdoor class with or without illuminator. Based on the identification results, it is concluded that the developed MFSDF face matcher outperformed the baseline face matchers for both the cross- scenarios.

- Based on the experimental results, it is concluded that, (i) CNN can be used to classify the data in terms of indoors or outdoors, with or no glass and with or without the usage of active illumination, when using both constrained and unconstrained face datasets in the 
SWIR band. The grouping of the datasets in terms of indoors or outdoors, with or without glass and active illumination can provide significant improvement for face recognition in forensic applications.

\subsubsection{Evaluation for Single Sensor Multi-Wavelength Database}

In this work, the challenges of face recognition in the SWIR band are investigated for the face images collected in heterogeneous environments i.e. when the face images are captured using multi-spectral imaging system against the visible (good quality) images.

- A deep convolutional neural network is developed to automatically categorize face data captured under various challenging conditions, before the FR algorithms are used. The CNN model is trained using the challenging SWIR face database and, for each classification level, a series of tests are performed to select the network parameters that result in high classification accuracy.

- Based on the experimental results, it is concluded that CNN provided with significantly improved classification accuracy, more than $90 \%$ for the classification based on individual subjects (Scenario 2) and more than $80 \%$ for the set with all data (Scenario 2). The impact of grouping of the database is investigated and based on experimental results it is concluded that using grouping of the datasets results in significant improvement in the rank-1 identification accuracy for each cross-spectral scenario. For class with $1550 \mathrm{~nm}$ label, there is a improvement in the rank identification accuracy from $66 \%$ to $87 \%$ (from rank 1 to rank 5).

- Finally, it is demonstrated that for face images captured at $1550 \mathrm{~nm}$, high identification rates are obtained. This conclusion is particularly important for unconstrained FR scenarios, as the wavelength is eye safe and is preferred over other wavelengths for long stand of distances in night time environments.

- Based on the experimental results, it is concluded that: (i) CNN can be used to classify the data in terms of wavelength for both constrained and unconstrained face datasets in 
CHAPTER 9. MULTI-SCENARIO AND MULTI-WAVELENGTH SWIR BASED FACIAL RECOGNITION SYSTEM

the SWIR band. The grouping of the datasets in terms of individual wavelengths can provide significant improvement for face recognition in surveillance applications. 


\section{Chapter 10}

\section{Multi-Sensor based Facial Recognition}

\section{System}

To conduct this study, a unique multi-sensor database (DB1), using Samsung Zoom, Nokia 1020, iPhone 5S and Samsung S5, was collected containing face images indoors, outdoors, with yaw angle from $-90^{\circ}$ to $+90^{\circ}$ and at two distances of 1 and 10 meters [23] as discussed in Chapter 3. The main challenge was the automatic image filtering of multi-sensor database collected using different type of cell phone devices (Samsung Zoom, Nokia and iPhone), indoors, outdoors and at variable standoff distances. In this chapter, the deep learning based developed hierarchical classification network is discussed.

To deal with the problem for pose variations, face detection and pose estimation algorithms were used for classifying the facial images into a frontal or a non-frontal class. The complete pipeline for the developed system is shown in Fig. 10.1 .

The hierarchical classification of the data was performed as follows:

- Level 1, face images were classified based on cell phone type.

- Level 2, face images were further classified into indoor and outdoor images.

- Level 3, face images were classified into close (1m) and far, low quality, (10m) distance. 


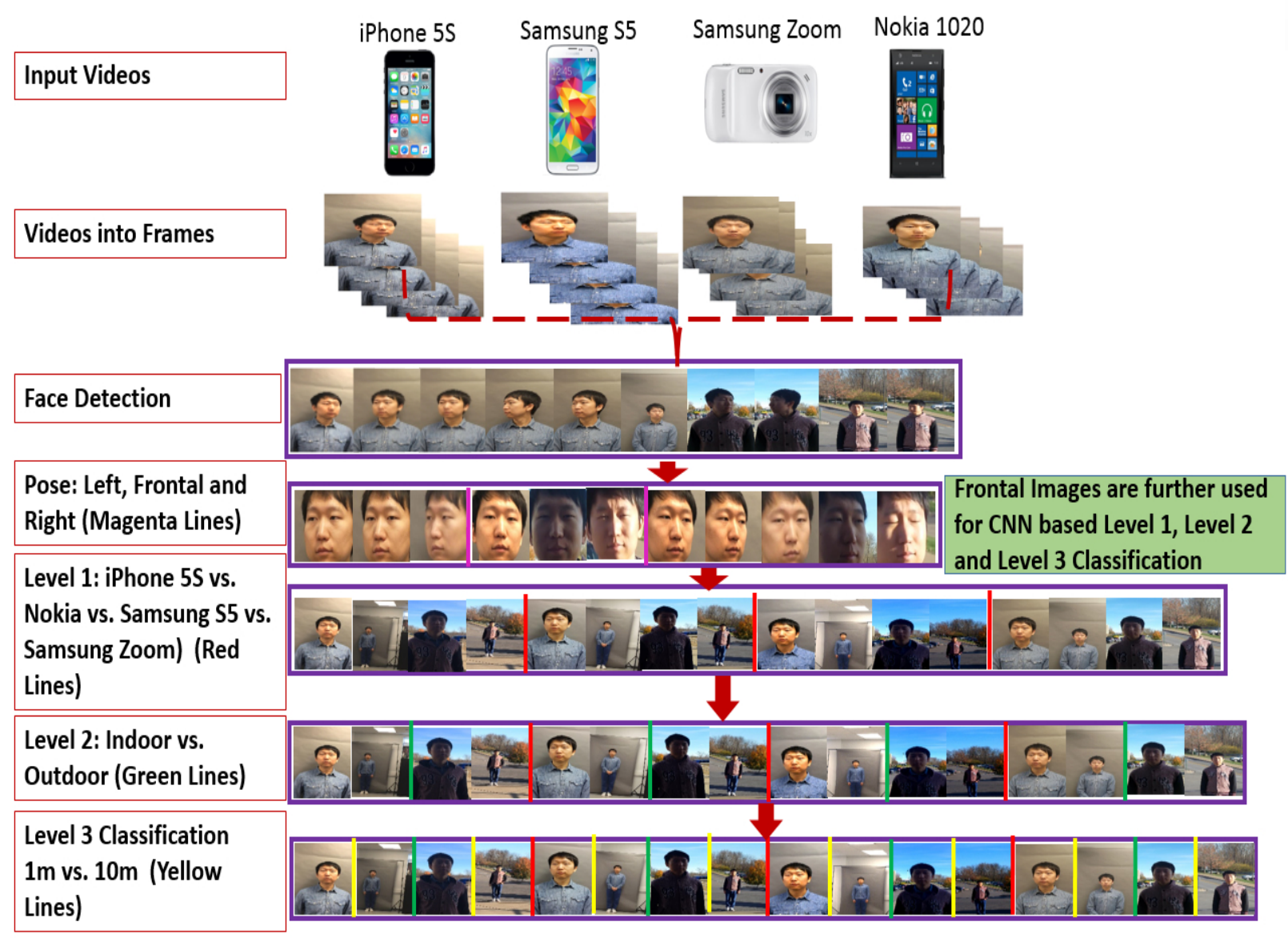

Figure 10.1: An overview of the developed hierarchical classification approach for the face images captured from the mobile phones under various conditions. Please note that after performing the first three steps (video into frames, face detection and pose estimation), the frontal face images were selected to perform the classification starting from top (Level 1) to bottom (Level 3). [23]

\subsection{Classification from Developed CNN Architecture}

A scenario dependent and sensor adaptive CNN network was developed, which is capable of classifying test images with class label of phone type, illumination condition and standoff distance (see in Fig. 10.2). The lower layer features are favorable for landmark location and pose estimation [57]. Whereas, the higher layer features are best fit to perform the classification task. This work is focused on higher layer features to perform the hierarchical classification. The network consists of convolutional layers, followed by pooling layers (max) and rectification layers, such as rectified linear unit (ReLU) along with fully connected layers as discussed in Chapter 4. Finally, the output was fed to Softmax layer that assigns a label for each class. For level 1 classification, the last Softmax layer assigns a label of phone type (iPhone 5S or 
Samsung galaxy S5 or Samsung zoom or Nokia). For level 2 classification, the last Softmax layer assigns a label of indoor or outdoor. Finally, for level 3 classification, Softmax provides a label close or far distance (see in Fig. 10.2).

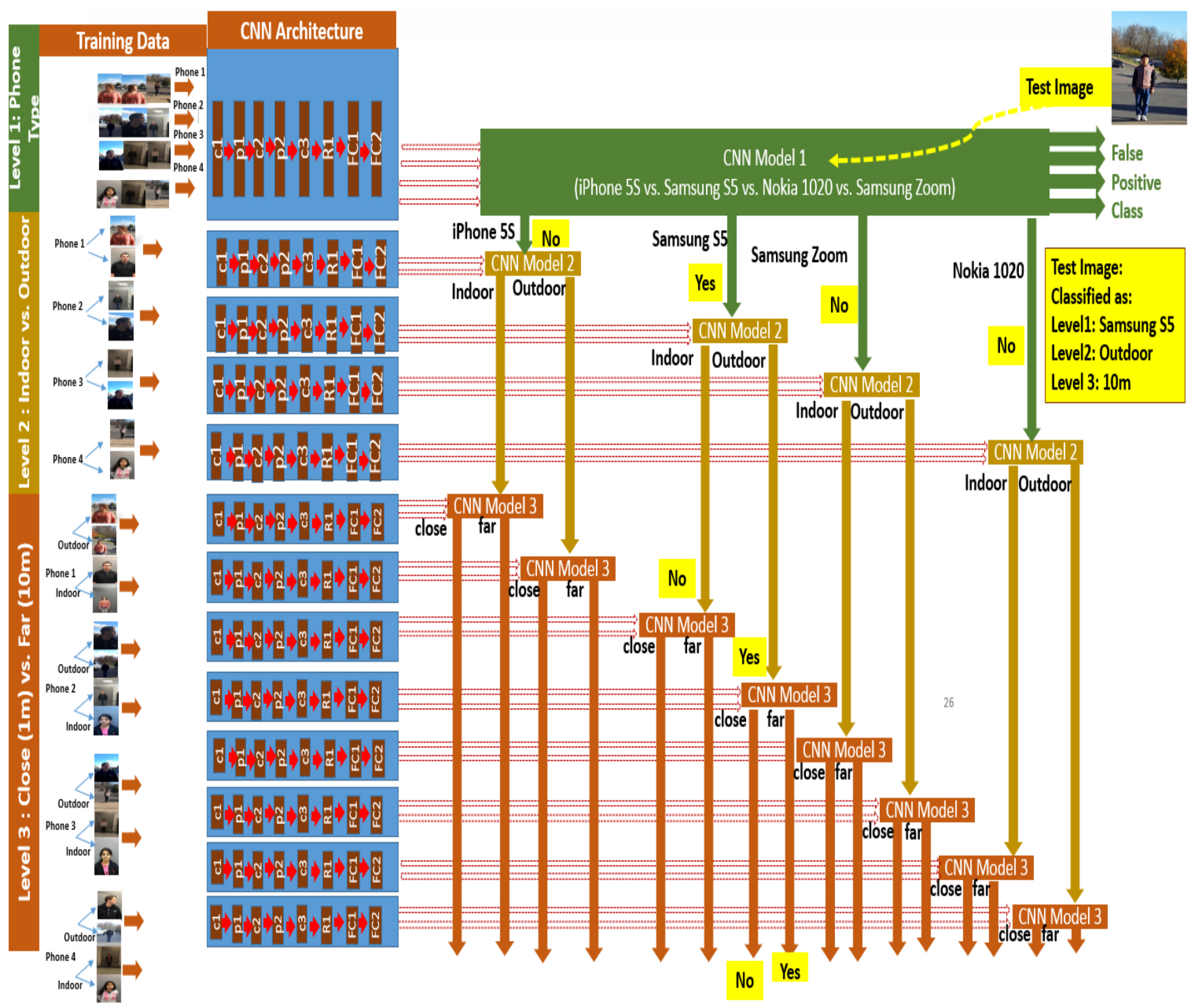

Figure 10.2: Developed CNN scheme to perform hierarchical classification. In CNN architecture: C represents the convolution layer, $\mathrm{P}$ the pooling layer, $\mathrm{R}$ the rectification layer and FC for the fully connected layer. The number represents the layer number for example, $\mathrm{C} 1$ represents the first convolution layer. CNN architecture consists of three convolution layers (C1, C2 and C3), two pooling layers (P1 and P2), one rectified layer (R1) and two fully connected layers (FC1 and FC2). 


\subsubsection{Training and Testing}

For the limited amount of training data, CNN pre-trained on large databases (ImageNets) is used by the researchers for the recognition and classification tasks [57]. In this work, to collect a large training database from available image repositories and to label the database manually is a time consuming process. There was no pre-trained multi-sensor network model available to use for the $\mathrm{CNN}$ network, therefore the models were trained on the original database for each level (level 1 to level 3). To train the system for each level, a fixed value of 0.92 for the momentum parameter, a batch size of 100 and a learning rate of 0.002 was selected. The classification framework was performed for 13 different set of epoch values, namely $4,8,12,16, \ldots ., 52$ for each level of the classification from level 1 to level 3. The classification results are presented in the experimental results section.

- Level 1: The input face database consists of images collected under variation in illumination conditions, standoff distances, sensor types, ethnicity and gender. To train the system, 4 labeled classes with phone type were used to train the system. The network was trained to classify each of the test images into the right phone type face image (e.g. face images collected using an iPhone, were categorized into the iPhone face folder).

- Level 2: To train the system, both indoor and outdoor face images were selected for each phone. Thus, the original face data categorized into a phone type face folder (Level 1), were further categorized into either an indoor or outdoor category.

- Level 3: To train the system, both $1 \mathrm{~m}$ and $10 \mathrm{~m}$ face images were selected for indoor and outdoor class. The data used for training the level 2 classifier was now further classified into either $1 \mathrm{~m}$ or $10 \mathrm{~m}$ distance category.

\subsubsection{Level 1: CNN based Phone Type Classification}

In this, $\mathrm{CNN}$ network was developed for the grouping of the database into four classes with labels iPhone 5S, Samsung Zoom, Nokia 1020 and Samsung S5. To train the CNN network, three scenarios were selected: 
- Scenario 1, the subjects in the training and test sets were different, and the images were taken at different locations and days. The database DB2 (collected outdoors at standoff distance of 2.5 to 80 meters) was used for the training and DB1 for testing.

- Scenario 2, DB1 is selected for training (50\%) and the rest of the database was used for testing without any overlap of subjects.

- Scenario 3, the images collected from both DB1 (50\%) and DB2 (All Data) were used for training, while the DB1 for testing. There was no overlap of subjects for training and testing.

Table 10.1 depicts the accuracy results for the grouping of data in terms of phone types from $\mathrm{CNN}$ used. Based on the results, it is concluded that the classification results of the highest accuracy were achieved from scenario 3 and the classification accuracy reaches almost $75 \%$.

Table 10.1: Classification results from CNN: Phone Type. DB1 consists of 100 subjects and DB2 consists of 80 subjects.

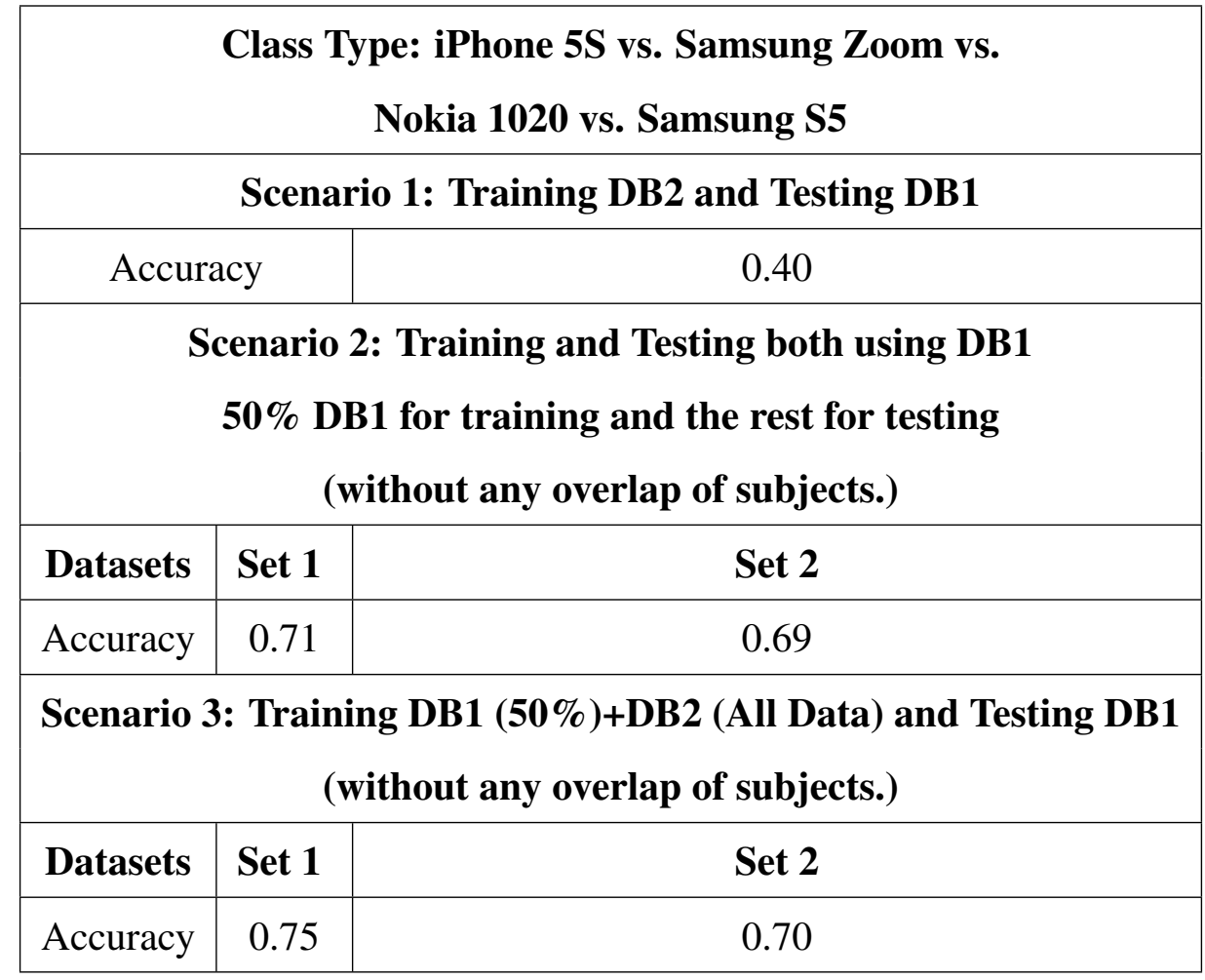




\subsubsection{Level 2: CNN based Conditional Classification}

For conditional class, the labeled database with phone type from level 1 classification were further classified into indoor and outdoor face images. To train the CNN network, the data was divided into different set sizes $(10 \%, 20 \%, 30 \%, 40 \%$ and $50 \%)$ for training, while the rest of the data was used for testing. In order to examine the effectiveness of the classification system, this process was repeated five times, where each time a different training set was randomly selected and rest of the data was used for testing (without overlap of subjects).

Classification was performed for 13 different sets of epoch values $4,8,12, \ldots ., 52$ for each phone and, finally, the value where the highest classification accuracy was achieved was selected from all five sets. The results for Samsung S4 Zoom phone are represented in Fig. 10.3, where the highest classification accuracy was achieved for the epoch value of 16 and $50 \%$ of the data was used for training.

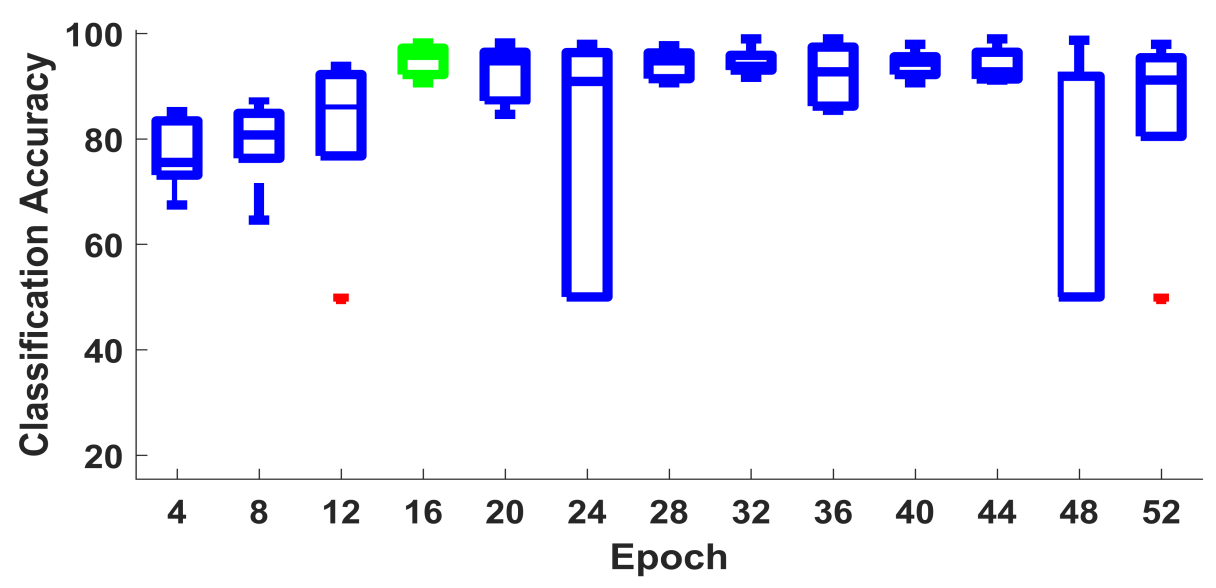

Figure 10.3: Classification accuracy vs. Epoch after running a set of five experiments.

In Table 10.3, classification results are presented with the epoch value which resulted in the highest accuracy for Samsung S4 Zoom and iPhone 5S. The same set of experiments were repeated for Samsung S5 and Nokia 1020. Based on the results, it was concluded that the classification results of the highest accuracy were achieved when $50 \%$ of the data was used for training. For Samsung Zoom, Nokia 1020 and Samsung S5, the classification accuracy on average from 5 sets reaches greater than $93 \%$. For iPhone $5 \mathrm{~S}$, the classification accuracy on average reaches more than $91 \%$. In Table 10.2, the classification results of the highest accuracy from each phone are presented. Based on mean and variance plots, it is concluded that the 
classification accuracy reaches approximately 95\% when the Nokia 1020 face dataset was used (this was the highest accuracy compared to any other phone specific face dataset used see Fig. 10.4).

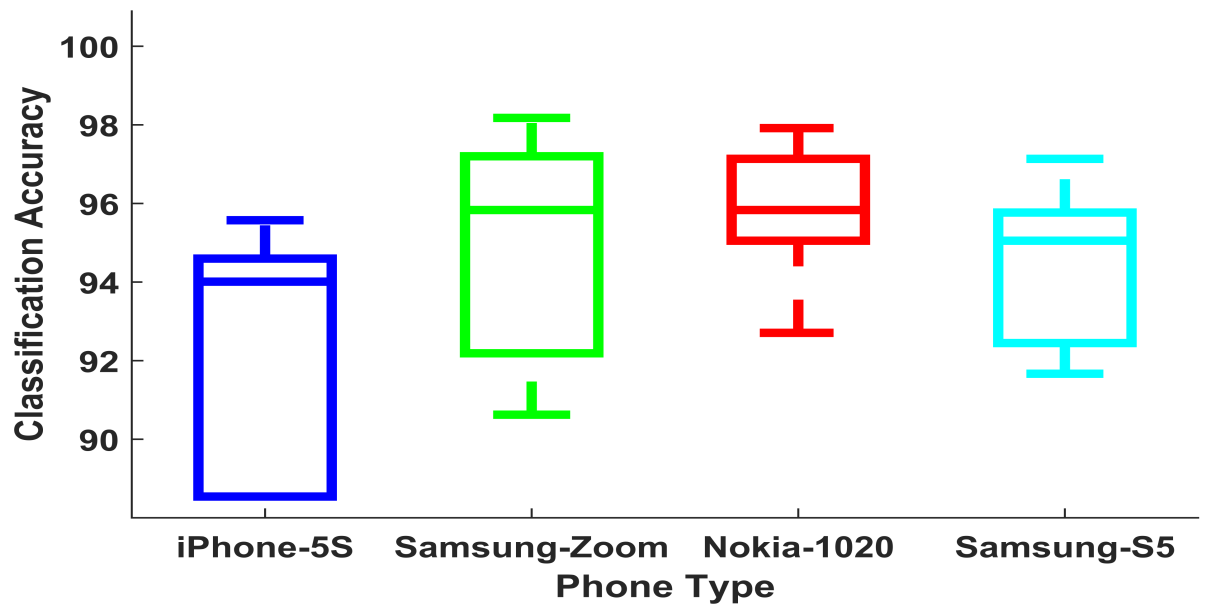

Figure 10.4: Classification accuracy results with a selected set of epoch and training sets for CNN. Each boxplot is based on results from 5 randomly selected training and testing sets.

Table 10.2: The best classification results from CNN for all the phones: Indoor vs. Outdoor.

\begin{tabular}{|c|c|c|c|c|}
\hline $\begin{array}{c}\text { Phone } \\
\text { Type }\end{array}$ & $\begin{array}{c}\text { iPhone } \\
\text { 5S }\end{array}$ & $\begin{array}{c}\text { Samsung } \\
\text { S5 }\end{array}$ & $\begin{array}{c}\text { Nokia } \\
\mathbf{1 0 2 0}\end{array}$ & $\begin{array}{c}\text { Samsung } \\
\text { Zoom }\end{array}$ \\
\hline & 95.57 & 97.13 & 97.91 & 98.17 \\
\hline
\end{tabular}

\subsubsection{Level 3: CNN based Standoff Distance Classification}

For standoff distance classification, the labeled indoor and outdoor data from level 2 classification was used to classify into either a close or a far distance class. The classification experiments were performed for 13 different set of epoch values of $4,8,12, \ldots, 52$. In Table 10.4 the highest classification accuracy results are presented for all the phones.

Based on the results, it is concluded that for Samsung S4 Zoom, for both the indoor and outdoor class, the classification accuracy reaches greater than 94\%. For iPhone 5S, with the indoor class, the classification accuracy reaches greater than $90 \%$ and for the outdoor class, the classification accuracy reaches almost 60\%. For Samsung S5, with the indoor class, the 
Table 10.3: Classification results from CNN: Indoor vs. Outdoor

\begin{tabular}{|l|l|l|l|l|l|}
\hline Datasets & set 1 & set 2 & set 3 & set 4 & set 5 \\
\hline \multicolumn{7}{|c|}{ Samsung Zoom } \\
train 10\% & 78.05 & 70.35 & 80.52 & 50.0 & 82.70 \\
train 20\% & 86.84 & 90.39 & 83.55 & 91.56 & 87.87 \\
train 30\% & 88.97 & 50.00 & 89.71 & 89.34 & 94.67 \\
train 40\% & 96.34 & 90.09 & 85.34 & 94.83 & 92.67 \\
train 50\% & 95.83 & 92.71 & 96.88 & 98.17 & 90.63 \\
\hline \multicolumn{7}{|c|}{ iPhone 5S } \\
train 10\% & 87.99 & 50.00 & 50.00 & 50.00 & 84.21 \\
train 20\% & 50.00 & 50.00 & 90.13 & 89.80 & 50.00 \\
train 30\% & 92.85 & 93.57 & 91.91 & 90.71 & 50.00 \\
train 40\% & 94.17 & 50.00 & 91.13 & 92.89 & 93.97 \\
train 50\% & 94.27 & 88.54 & 88.54 & 95.57 & 94.01 \\
\hline
\end{tabular}

classification accuracy reaches greater than $94 \%$ and for the outdoor class, the classification accuracy reaches greater than $75 \%$. Based on mean and variance plots, the highest classification accuracy results for the indoor dataset that was classified into close or far distance were achieved for Nokia 1020. For the same classification problem, while using outdoor dataset, the highest accuracy classification results were achieved when using the Samsung S4 Zoom (see Fig. 10.5).

In Table 10.5, the results are presented for two scenarios, where for scenario 1, the original raw images were selected, and for scenario 2 the detected face images were selected to perform the classification using CNN. Based on the results, it is concluded that overall, the highest classification accuracy results were achieved when the original database was selected to perform the classification. For the indoor class, the results are similar. However, for the outdoor class, scenario 1 outperformed scenario 2 . There is a valid reason for the outcome results: for the outdoor dataset, the images with background have more features to perform the classification, while, for the indoor class the background is uniform. 


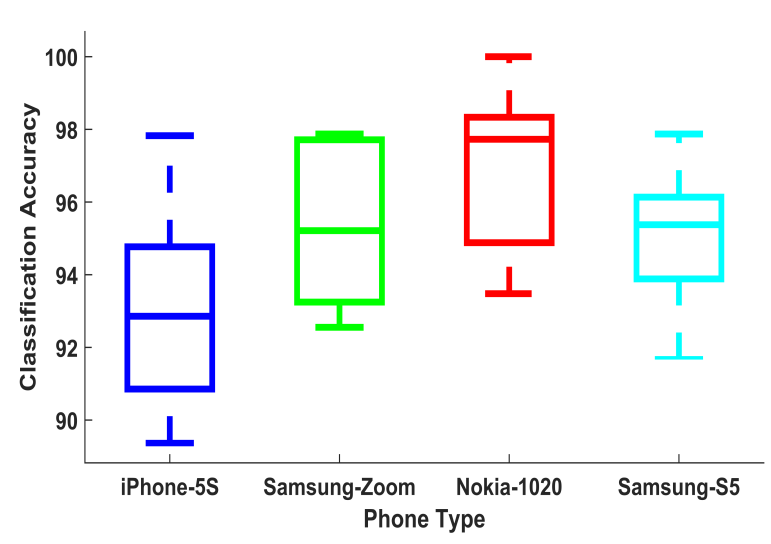

(a) Indoor Class: Close vs. Far Distance

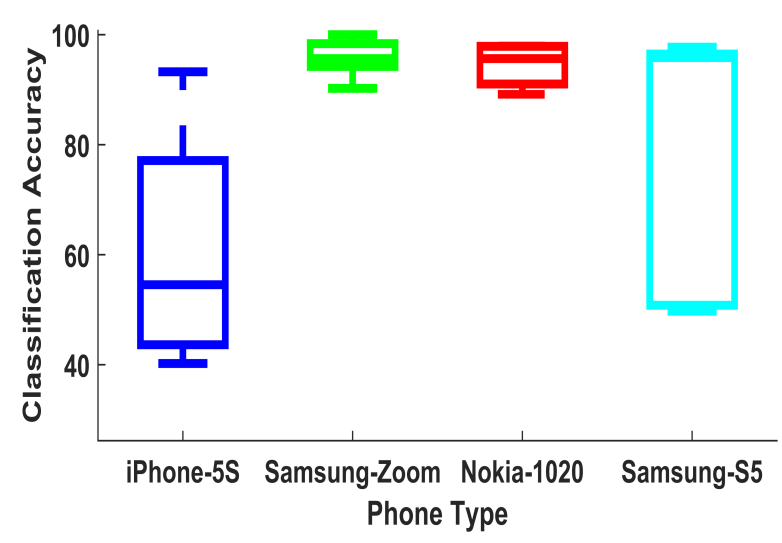

(b) Outdoor Class: Close vs. Far Distance

Figure 10.5: Classification results for Level 3 classification for all the phones.

Table 10.4: Classification accuracy results from CNN (\%): Close (1m) vs. Far (10m) distance.

\begin{tabular}{|c|c|c|c|c|c|}
\hline Datasets & set 1 & set 2 & set 3 & set 4 & set 5 \\
\hline \multicolumn{6}{|c|}{ Samsung Zoom } \\
Indoor & 97.87 & 97.66 & 95.21 & 92.55 & 93.48 \\
Outdoor & 95.56 & 97.82 & 95.65 & 100.00 & 90.24 \\
\hline \multicolumn{5}{|c|}{ iPhone 5S } \\
Indoor & 93.75 & 97.83 & 92.86 & 89.36 & 91.35 \\
Outdoor & 71.73 & 93.25 & 44.78 & 40.22 & 54.54 \\
\hline \multicolumn{5}{|c|}{ Nokia 1020 } \\
Indoor & 100.00 & 93.48 & 97.78 & 97.73 & 95.35 \\
Outdoor & 97.87 & 95.65 & 91.67 & 97.92 & 89.13 \\
\hline \multicolumn{7}{|c|}{ Samsung S5 } \\
Indoor & 97.87 & 94.62 & 95.38 & 91.67 & 95.56 \\
Outdoor & 96.06 & 49.73 & 95.83 & 51.16 & 97.72 \\
\hline
\end{tabular}

\subsection{Face Matching Results: With and Without Grouping}

\subsubsection{Classification of Frontal vs. Non-Frontal}

Face recognition and image classification systems perform well when dealing with fullfrontal face images. In this work, the collected database consists of face images with different 
Table 10.5: Highest classification accuracy results from CNN: Close (1m) vs. Far (10m) distance.

\begin{tabular}{|c|c|c|c|c|}
\hline $\begin{array}{c}\text { Phone } \\
\text { Type }\end{array}$ & $\begin{array}{c}\text { iPhone } \\
\text { 5S }\end{array}$ & $\begin{array}{c}\text { Samsung } \\
\text { S5 }\end{array}$ & $\begin{array}{c}\text { Nokia } \\
\mathbf{1 0 2 0}\end{array}$ & $\begin{array}{c}\text { Samsung } \\
\text { Zoom }\end{array}$ \\
\hline \multicolumn{5}{|c|}{ Scenario 1: With Raw Database } \\
\hline Indoor & 97.83 & 97.87 & 100.00 & 97.87 \\
\hline Outdoor & 93.25 & 97.72 & 97.92 & 100.00 \\
\hline \multicolumn{5}{|c|}{ Scenario 2: With Detected Face Database } \\
\hline Indoor & 95.23 & 91.12 & 98.40 & 92.02 \\
\hline Outdoor & 89.20 & 92.83 & 93.08 & 91.01 \\
\hline
\end{tabular}

poses. In order to keep only full-frontal face images to perform the classification experiments, an automated method was selected for the detection and estimation of face pose [110]. The classification was performed into frontal and non-frontal (for all the phones, indoors, outdoors, at close and far distance) based on the automated estimated pose angle.

- Face Detection: In this work, cascaded adaboost classifier was used for face detection [111] and the algorithm was adapted for the challenging multi-sensor database collected in our lab. The good results were achieved for the face images collected at close distance for indoors and outdoors, as shown in Fig. 10.6. The main challenge was to detect the face for the images collected outdoors, at far distance, with some pose angle and for the phone camera with low pixel quality. For some cases, no output or multiple outputs with false positives and false negatives were detected as shown in Fig. 10.7. To address the issue, for the output with multiple images, once the bounding boxes were found, the condition to search for a face bounding box was applied based on the size of box and the pixel location.

- Pose Estimation: There are three types of face pose rotation angles such as yaw, pitch and rolling angle [112]. For the database collected under un-controlled conditions, the algorithm developed by Aghajanian et al. [110] was used to estimate the face pose. The detected face images were classified into three categories, left profile, frontal and right 


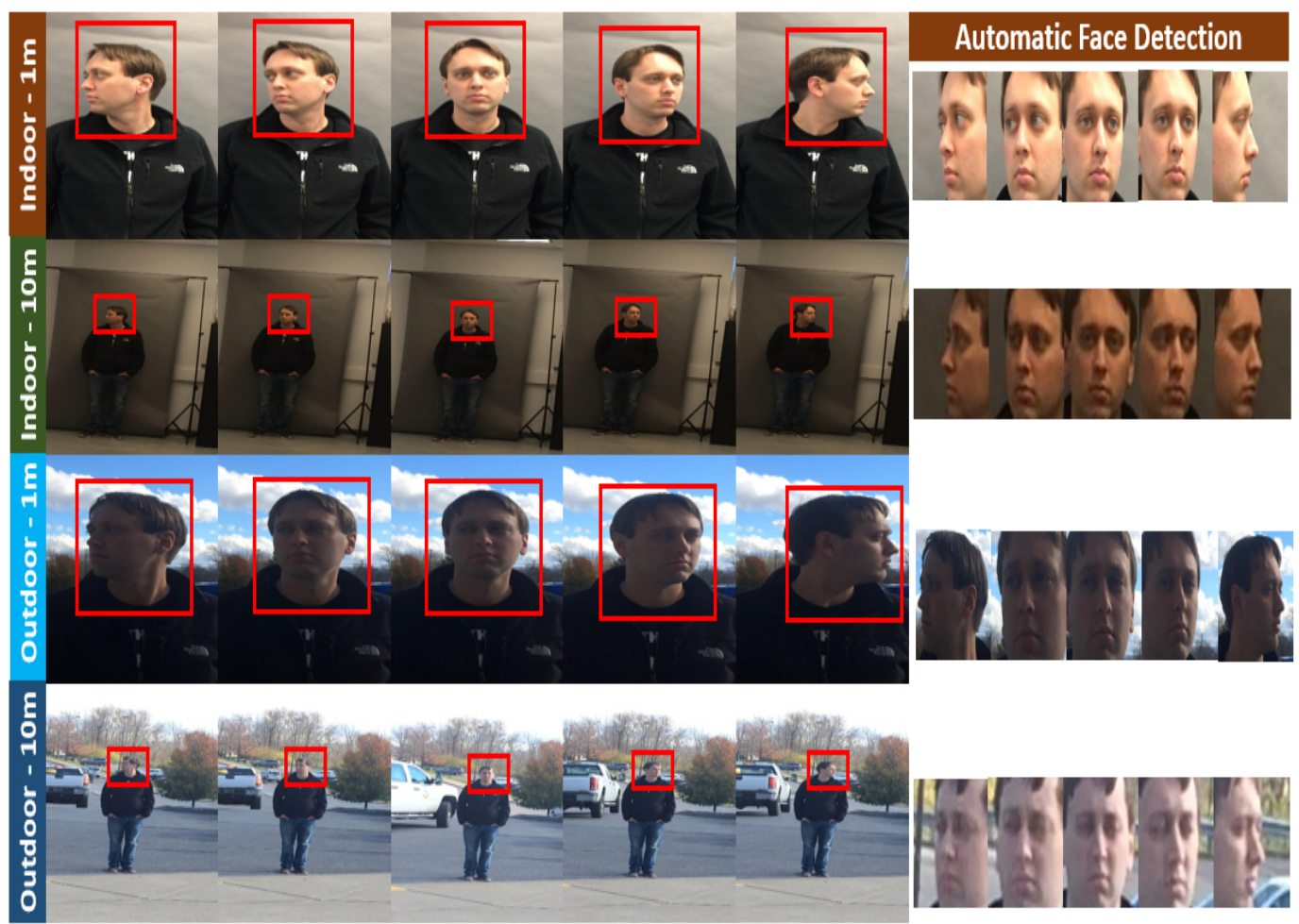

Figure 10.6: Face detection for the face images captured with the mobile phones under un-constrained conditions.

profile, with yaw angle from $-90^{\circ}$ to $90^{\circ}$.

Next, a probabilistic framework was generated, where the subject's face was represented by non-overlapping grid of patches and generative model based on this patch representation was further used for pose estimation on the test images. To perform these experiments, the radial bias functions $(\mathrm{RBF})$ was selected with size of RBF9D, patch grid resolution of $10 \times 10$, number of patches of 100 , and standard deviation for RBF of 45. The good results are achieved for the database collected from sensors with large size face images (using Samsung S4 Zoom and Nokia 1020) as shown in Fig. 10.8. It was more challenging to process face images of smaller spatial resolution (using the iPhone, Samsung S5). For the outdoor data at far distances, some of the faces were mis-classified with a wrong pose angles. The face images with frontal view and yaw angle of $0^{\circ}$ were classified as frontal and face images with left and right profile were classified as non-frontal (yaw angle less than and greater than $0^{\circ}$ ). 


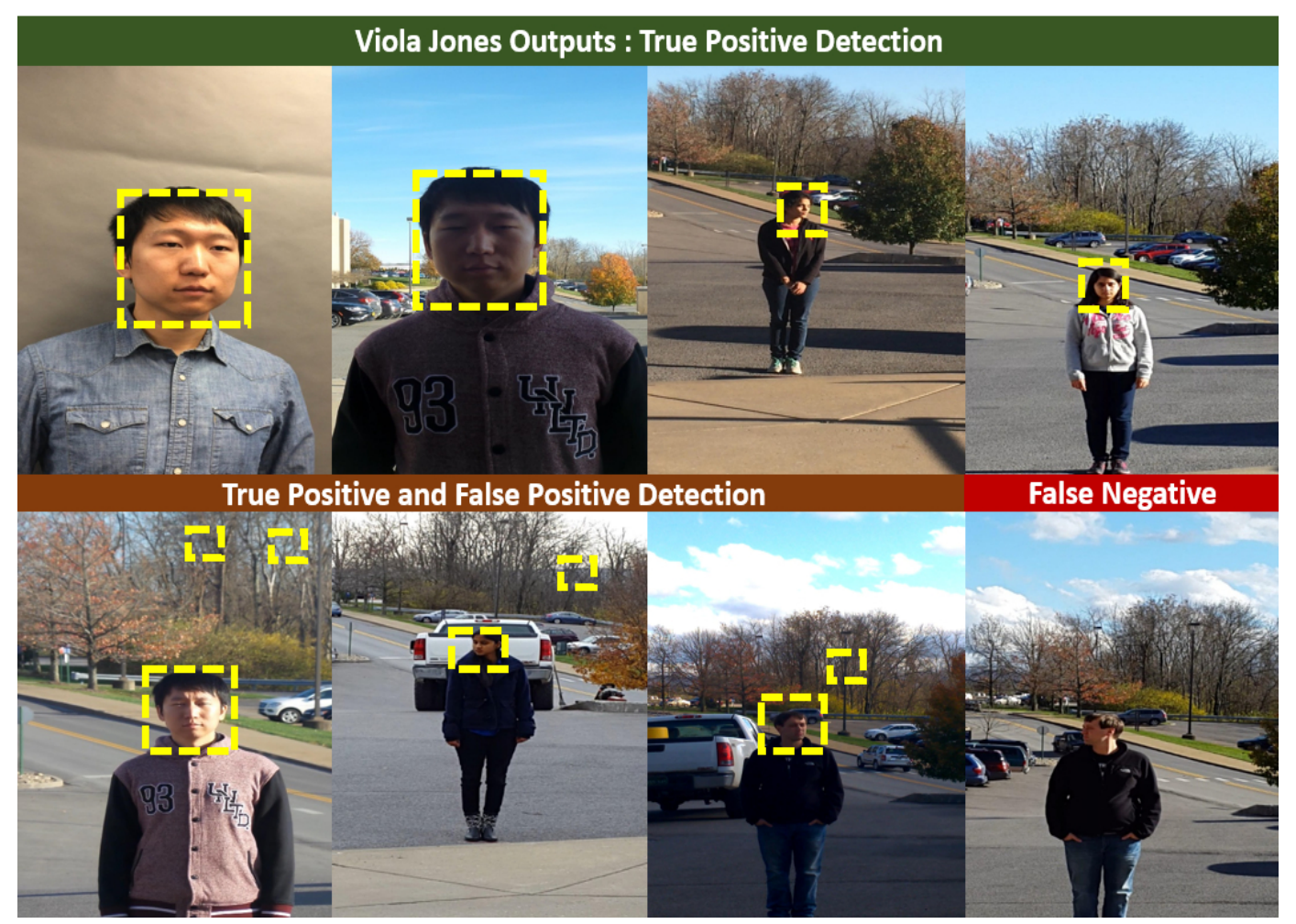

Figure 10.7: Viola Jones detector with True Positive, False Positive and False Negative results.

\subsubsection{Face Matching}

Local Binary Patterns: LBP matcher was used to extract the appearance and texture information from human faces and is invariant to changes in illumination conditions.

VGG Face Network: Although the VGG-Face network was trained on a specific set of identities, the features extracted in intermediate layers can be applied to other identities for recognition. To evaluate the dataset, the features extracted in the last fully connected layer before the Softmax as deep facial features (fc8 in [80]) were used. The feature vectors were compared using the Euclidean distance to generate a distance score between two face images.

\subsubsection{Face Matching Results: With and Without Grouping}

First, experiments were performed with the original FR system, namely when no grouping was used. Second, grouped datasets were used in terms of cell-phone type, indoors or outdoors and close or far distance. The identification results using LBP-CHI method are summarized in Table 10.6. Based on the results, it is determined that the rank-1 identification accuracy improved from 53\% (All Data) to 66\% - Level 1 (Samsung S4 Zoom), to 82\% - Level 2 (Sam- 


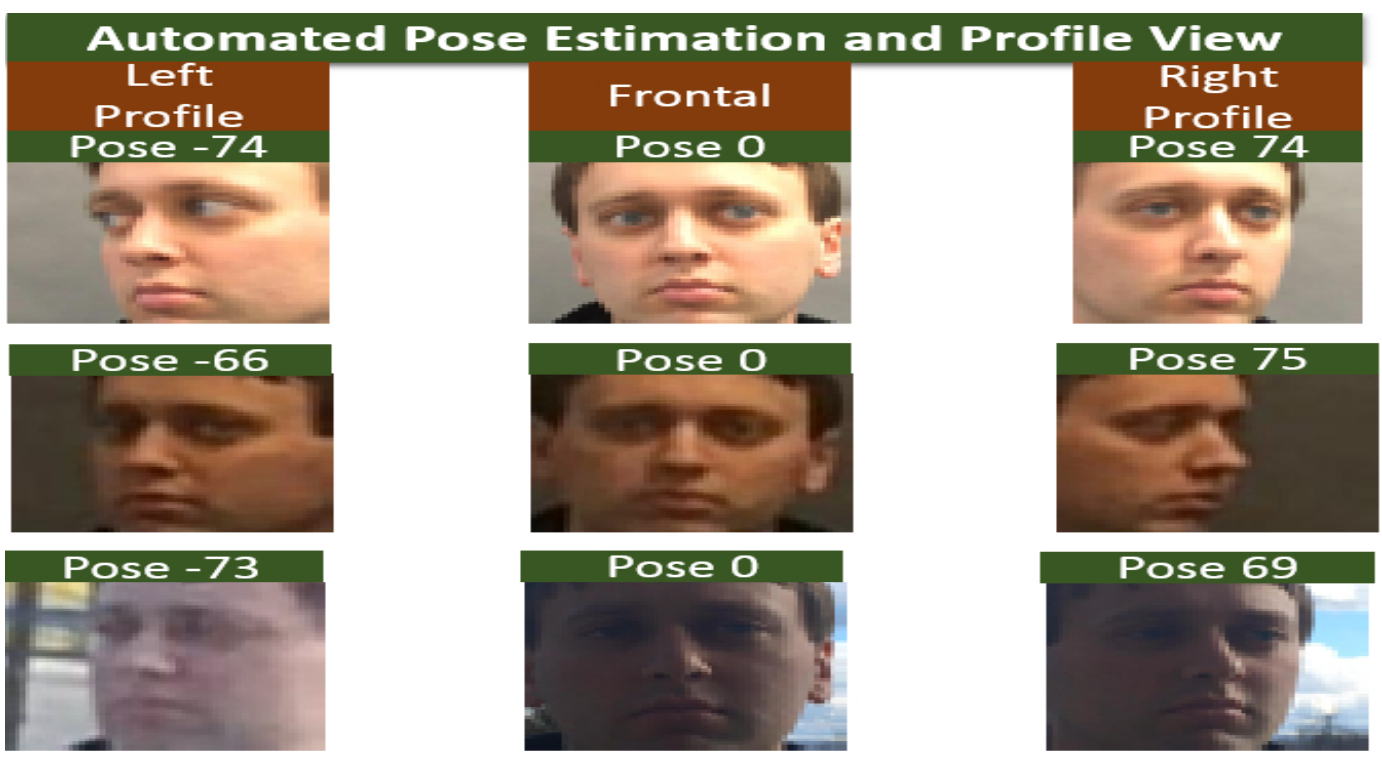

Figure 10.8: Pose estimation from $-90^{\circ}$ to $+90^{\circ}$ angle for the detected face images.

Table 10.6: Face matching for with and without grouping of data.

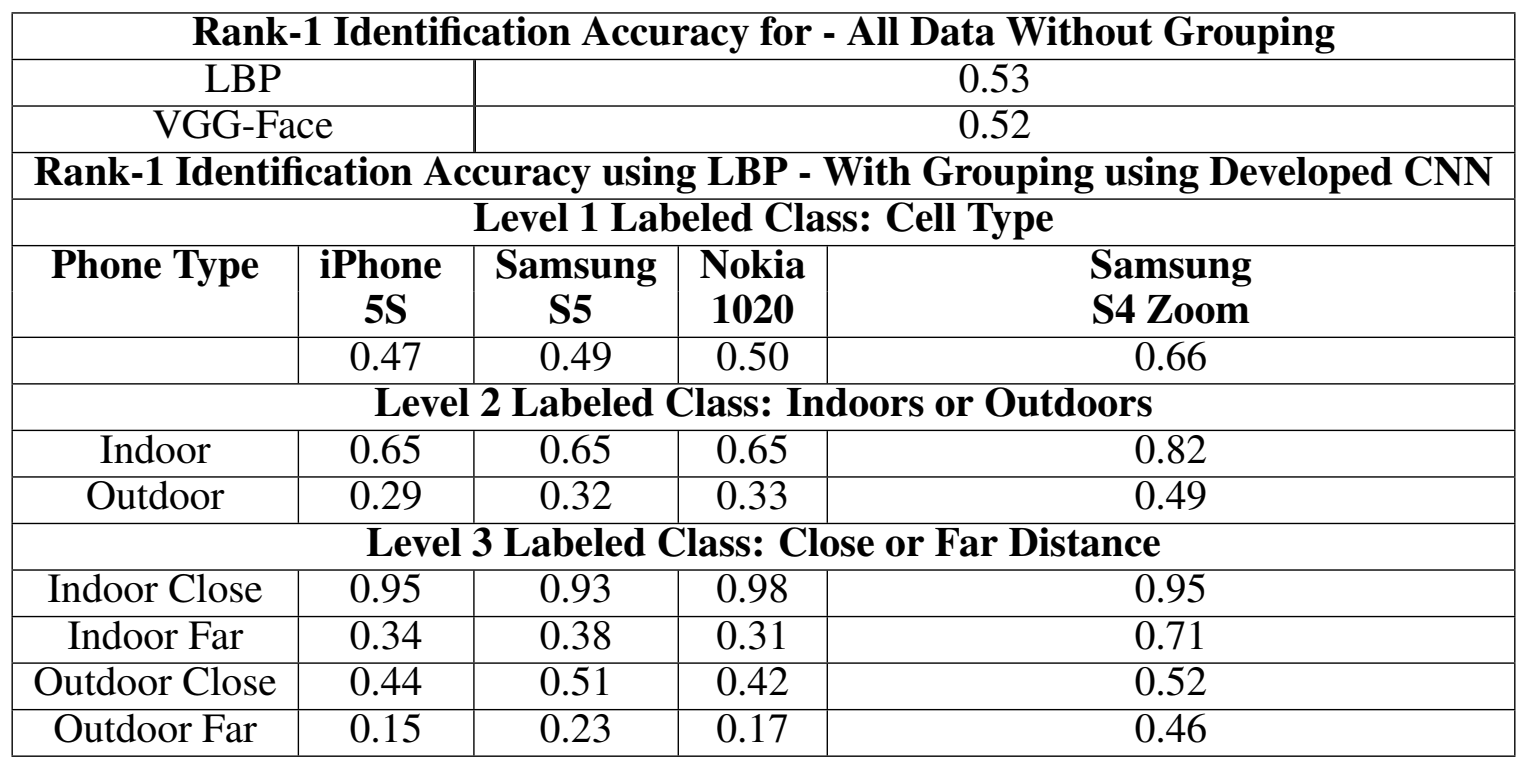

sung S4 Zoom Indoor) and to 95\% - Level 3 (Indoor Close) and to 71\% (Indoor Far). The face matching experiments are represented in Table 10.6. To show the impact of grouping or pre-screening of the database on face matching, the first 5 rank identification rates are shown in Table 10.7 
Table 10.7: Impact on Face Matching Accuracy when either of Data Grouping or Databased Pre-Screening was used

\begin{tabular}{|c|c|c|c|c|c|}
\hline Identification Accuracy & Rank 1 & Rank 2 & Rank 3 & Rank 4 & Rank 5 \\
\hline \multicolumn{6}{|c|}{ Face Matching - Without Grouping } \\
\hline LBP & 0.53 & 0.61 & 0.66 & 0.70 & 0.73 \\
\hline VGG-Face & 0.52 & 0.59 & 0.63 & 0.66 & 0.68 \\
\hline Face Matching - With Grouping (Level 1) \\
\hline LBP & 0.66 & 0.74 & 0.78 & 0.80 & 0.82 \\
\hline VGG-Face & 0.68 & 0.74 & 0.78 & 0.80 & 0.83 \\
\hline
\end{tabular}

Table 10.8: Classification results for extended database (100 subjects) from CNN for all the phones: Indoor vs. Outdoor.

\begin{tabular}{|c|c|c|c|c|}
\hline $\begin{array}{c}\text { Phone } \\
\text { Type }\end{array}$ & $\begin{array}{c}\text { iPhone } \\
\text { 5S }\end{array}$ & $\begin{array}{c}\text { Samsung } \\
\text { S5 }\end{array}$ & $\begin{array}{c}\text { Nokia } \\
\mathbf{1 0 2 0}\end{array}$ & $\begin{array}{c}\text { Samsung } \\
\text { S4 Zoom }\end{array}$ \\
\hline CNN-1 & 90.66 & 90.23 & 90.00 & 93 \\
\hline CNN-2 & 95.23 & 95.33 & 96.33 & 97 \\
\hline
\end{tabular}

\subsubsection{Classification and Matching: With Extended Database}

In this, the database size was doubled in terms of all scenarios. The classification (Level 2) and face matching experiments were repeated. For the classification where $50 \%$ of the data was used for training and the rest of the data was used for testing (without any overlap of subjects), 93\% accuracy is achieved for Samsung Zoom. To further improve the classification results, the model was selected with more layers (CNN-2: 12 layers based on MatConvNet) and the classification reaches to $97 \%$ as shown in Table 10.8 .

Based on the face matching results, it is determined that the rank-1 identification rate improved from 82\% - VGG Face and 80\% - LBP (All Data) to more than 98\% - Level 2 (All Phones Indoors 1 meters) as shown in Table 10.9 .

\subsection{Conclusion}

The advantages and limitations of the developed multi-sensor system were investigated. The mobile phone adapted convolutional neural network based, hierarchical classification framework was designed to automatically categorize the face images captured under various challenging conditions, before the FR algorithms are used. 
Table 10.9: Impact on Face Matching Accuracy when Data Grouping or Data based PreScreening was used for extended database (100 subjects).

\begin{tabular}{|c|c|c|c|c|c|}
\hline Rank-1 Identification Accuracy - All Data Without Grouping for 1 meters \\
\hline Rank & Rank 1 & Rank 2 & Rank 3 & Rank 4 & Rank 5 \\
\hline LBP & 0.80 & 0.83 & 0.85 & 0.85 & 0.86 \\
\hline VGG-Face & 0.82 & 0.83 & 0.84 & 0.85 & 0.86 \\
\hline \multicolumn{5}{|c|}{ LBP Matcher for Database With Grouping } \\
\hline \multicolumn{5}{|c|}{ Level 2 Labeled Class: Indoors vs. Outdoors } \\
\hline Phone Type & iPhone & Samsung & Nokia & Samsung \\
Rank-1 Identification Accuracy & $\mathbf{5 S}$ & S5 & $\mathbf{1 0 2 0}$ & S4 Zoom \\
\hline Indoor & 0.99 & 1.00 & 0.99 & 1.00 \\
\hline Outdoor & 0.57 & 0.46 & 0.56 & 0.58 \\
\hline
\end{tabular}

- First, a multi-sensor database was collected (videos) indoors, outdoors, at close and far standoff distances and with different poses using iPhone, Samsung and Nokia phones.

- The performance of the image classification system is sensitive to face pose variations. An algorithmic approach for the selection of frontal face images was generated: first, faces were detected, next a pose estimation algorithm was applied, and, finally, based on the left, right and frontal view, the face images were classified into a frontal or a non-frontal (left and right profile) class. The frontal face image dataset generated was then used to apply the developed CNN framework for hierarchical classification (Level 1, Level 2 and Level 3).

- The CNN model was trained using the challenging multi-sensor mobile phone face database and, for each classification level, a series of tests were performed to select the network parameters that result in high classification accuracy. The experiments showed that for Level 1, the developed CNN provided with significantly improved classification results, i.e. the classification accuracy is more than $80 \%$. The classification accuracy is more than $95 \%$ for Level 2 classification in the most scenarios tested. Also, the classification accuracy is more than $96 \%$ for Level 3 classification for the indoor class into close or far distance. For the outdoor class into close or far distance, the classification accuracy is more than $90 \%$.

- The face matching results provide important evidence that data grouping in terms of 
cell-phone type, indoors or outdoors and close or short distance provide significant improvement in the rank-1 identification rate, e.g. the performance improved from $53 \%$ to $82 \%$ for Level 2 and to $95 \%$ for Level 3 (Indoor close) and 71\% (Indoor far) for database labeled as Samsung S4 Zoom based on the developed architecture.

- Experimental results showed that CNNs, when properly designed, can be a useful tool in multi-sensor mobile face recognition settings, even though a limited mobile dataset is used. 


\section{Chapter 11}

\section{Dissertation Conclusions}

\subsection{Conclusions}

In this dissertation, the research objective was to improve the heterogeneous face recognition performance when using challenging face databases. The recognition scenarios investigated involve matching probe face images - captured using different camera sensors, during night time, at variable standoff distances and behind tinted glass panels - against their visible (good quality) face counterparts. Thus, one of the main contributions of this dissertation is the design and development of an IR face imaging system that has the capability of delivering a confident face matching performance at challenging conditions. The specific face datasets used are listed below:

1. NIR face image dataset captured at night time and (variable) long range standoff distances.

2. SWIR face image dataset, where the face images are captured outdoors, indoors and/or through a set of tinted glass panels.

3. Multi-Wavelength SWIR multi-pose face image dataset - wavelengths ranging from 1150 to $1550 \mathrm{~nm}$ at $100 \mathrm{~nm}$ intervals.

4. Mobile face image dataset, where various cell phone types are used and the face images are captured indoors, outdoors, and at variable standoff distances and pose angles. 
After collecting the challenging heterogeneous face databases, a set of face-based identification studies were conducted when dealing with three different matching scenarios: (i) intra-distance and intra-spectral, (ii) cross-distance and intra-spectral, and (iii) cross-spectral and cross-distance.

A general description of these studies and their main conclusions is discussed below:

- Study 1, a multi-feature, scenario dependent, face matching fusion scheme (MFSDF) was developed that improves the baseline system performance established by using both academic and commercial matchers. Experimental results show that for night time imagery database in NIR band, the rank-1 identification accuracy improved from $16 \%$ (commercial), $88 \%$ (academic) to $93 \%$ using developed system when NIR images at 30m were compared against NIR images collected at 120m distance. For cross-spectral (VIS $1.5 \mathrm{~m}$ vs. NIR 30m), the rank-1 identification accuracy improved from 19\% (commercial), 48\% (academic) to $56 \%$ for the developed system. Based on the results it is concluded that the rank-1 identification accuracy improved using the developed fusion scheme for both the intra-spectral and cross-spectral scenarios.

For Multi-Scenario database and when intra-spectral (SWIR vs. SWIR) face matching scenarios were investigated, the rank-1 identification accuracy improved from $48 \%$ (academic) to $75 \%$ for the developed scheme. For cross-spectral (VIS vs. SWIR), the rank-1 identification accuracy improved from $7 \%$ (academic) to $67 \%$ using the developed scheme. For Multi-Wavelength database, for cross-spectral (VIS vs. SWIR at 1350 $\mathrm{nm}$ ), the rank-1 identification accuracy improved from 58\% (academic) to $81 \%$ for the developed method and 54\% to $88 \%$ when VIS images are compared against SWIR face images at $1550 \mathrm{~nm}$ wavelength.

- Study 2, a deep convolution neural network based scenario dependent and sensor adaptable architecture was developed. To perform the classification using deep learning based framework, an empirical optimization of parameters was performed (epoch, momentum and learning rate). The developed network automatically classifies the datasets into specific categories: 
1. Visible and multi-distance NIR face images were classified into ethnicity and gender groups.

2. Multi-Scenario database was classified into (i) indoors or outdoors, (ii) with glass or no glass, and, (3) with or without the usage of active illumination.

3. Multi-wavelength face data was classified into individual wavelength ranging from $1150 \mathrm{~nm}$ to $1550 \mathrm{~nm}$.

4. Multi-Sensor database was classified into (i) phone type, (ii) indoor and outdoor images, and (iii) close (1m) and far, low quality (10m) distance.

Based on an extensive set of face matching experiments, it is demonstrated that the FR performance improved significantly when the grouping of the database was performed based on the developed deep convolutional framework.

1. For NIR imagery database, with the usage of image filtering the face matching rank-1 identification accuracy improved from 56\% (All vs. All) to $75 \%$ for Female Asian class and to $82 \%$ for Male With Beard class. Using demographic filtering of the data, where the data is filtered based on gender class (male and female), it is shown that male class provides improvement in the results for cross-distance and cross-spectral scenarios.

2. For Multi-Scenario database in SWIR band, the face matching rank-1 identification accuracy when using all data is $67 \%$ vs. $96 \%$ when data is automatically filtered into a face dataset where face images were captured indoors and where no tinted glass was used.

3. For Multi-Wavelength database in SWIR band, class with $1550 \mathrm{~nm}$ label, there is a significant improvement in the rank-1 identification accuracy from $66 \%$ to $87 \%$.

4. For Multi-Sensor database, face matching results provide important evidence that the data grouping in terms of cell-phone type, indoors or outdoors and close or short distance provide significant improvement in the rank-1 identification rate e.g. the performance improved from 53\% to $82 \%$ for Level 2 and to $95 \%$ for Level 3 (Indoor close) and 71\% (Indoor far) for database labeled as Samsung S4 Zoom. 
- Study 3, The face matching experiments resulted in better identification performance when operated at close standoff distance in night time environment. The experiments resulted in a performance that reduces as a function of distance (especially at 90m and $120 \mathrm{~m})$. To address this problem, an image restoration approach was developed and this approach was evaluated on NIR night time long range database under 5 different matching scenarios (i) Original Database and the following sub-sets (ii) Female Asian, (iii) Female Caucasian, (iv) Male With Beard and (v) Male Without Beard. Based on face matching experiments, it is demonstrated that for NIR database at $30 \mathrm{~m}$ distance, the rank-1 identification accuracy improved from $56 \%$ to $73 \%$ (All vs. All), to $75 \%$ for Female Asian class (Study1 followed by Study 2 and Study 3) and to $82 \%$ for Male With Beard class (Study1 followed by Study 2 and Study 3).

- Study 4, Statistical tests were conducted to find the statistical significance of incorporating 1 (developed fusion scheme), Study 2 (developed data filtering) and Study 3 (image restoration) to the face recognition system for the images captured in heterogeneous environments. A series of tests were conducted to find the most suitable type of statistical analysis test (parametric or non-parametric). Based on statistical analysis tests Friedman test (non-parametric) was selected for night time imagery database in NIR band. Statistical tests rejected the null hypothesis and showed the significance of incorporating fusion scheme, image restoration and stratification of the database before conducting the face matching studies. Moreover, face matching experiments were verified with statistical analysis results.

The reported results are beneficial in producing key trace evidence for the successful identification of individuals in forensics science and law enforcement applications in unconstrained FR scenarios, where imaging is performed indoors, outdoors, in night time and at variable long range distance. For the multi-wavelength database collected in SWIR band, it is demonstrated that when using face images captured at $1550 \mathrm{~nm}$, high identification rates are obtained. This conclusion is particularly important for unconstrained FR scenarios, where we may need to capture face images at $1550 \mathrm{~nm}$ (eye safe wavelength), at long distances and when operating at night time environments (preferable over other SWIR wavelengths). 


\subsection{Future Work}

Although in this dissertation, aforementioned developed algorithms significantly improve cross-spectral face matching performance when the database is collected under challenging conditions, beyond the concrete results and methods those were developed, there exists many avenues for future extensions.

- Deep networks are used for the classification of database collected under challenging conditions. To further improve the classifications results, more databases to train deep learning model as well as to test alternative $\mathrm{CNN}$ architectures can be included. However, the challenge is the complicated collection scenarios that require large resources when many subjects are involved. The developed $\mathrm{CNN}$ architecture can automatically provide the demographic information in terms ethnicity into Asian and Caucasian class. In future studies, more specific demographic strata can be used, e.g. in terms of ethnicity such as Hispanic, Middle Eastern, etc. can be investigated.

- Other factors, such as generating subsets based on facial expression, pose, age, etc. in the experiments can be investigated to further improve the performance of face recognition systems.

- One additional, challenge was to automatically locate the eye locations to normalize the face i.e. for multi-wavelength database when the face images are collected at $1450 \mathrm{~nm}$ wavelength, through tinted glass panels (Solarcool Gray-lite Glass Panel) [61, 72] in SWIR band and night time face images are collected outdoors at long standoff distances of $30 \mathrm{~m}$ to $120 \mathrm{~m}$. To address this problem, the eye locations were located manually. However, to make the face recognition more efficient, this process can be automated, or this process needs to become more efficient by developing more sophisticated algorithms that can be more robust on factor that affect eye localization performance such as, FR using deep learning networks. 


\section{List Of Publications}

\section{Peer Reviewed Journal Papers}

- N. Narang and T. Bourlai, "Face recognition in the SWIR band when using single sensor multi-wavelength imaging systems, Image and Vision Computing, Journal - Elsevier, vol. 33, pp. 26-43, Jan 2015.

- T. Bourlai, N. Narang and N. Mavridis, "On Designing Practical Long Range Near Infrared-based Face Recognition Systems", Elsevier - Image and Vision Computing Journal, May 2016.

- N. Narang and T. Bourlai,"Deep Features based Classification of Soft Biometric Traits when Matching Near-Infrared Long-range Face Images against their Visible”, Special Issue on Deep Learning for Computer Vision (Under Review).

- N. Narang and T. Bourlai, "Deep Networks based Classification in SWIR Band for Forensic Facial Recognition, Forensic Science International Journal, Elsevier (Submitted).

\section{Peer Reviewed Conference Papers}

- T. Bourlai, N. Narang et. al., "On Designing a SWIR Multi-Wavelength Facial-Based Acquisition System", SPIE (Vol. 8353), Infrared Technology and Applications XXXVIII, Baltimore, U.S.A., Apr. 2012.

- J. Ice, N. Narang, C. Whitelam, N. Kalka, L. Hornak, J. Dawson, and T. Bourlai, "SWIR Imaging for Facial Image Capture through Tinted Materials, SPIE (Vol. 8353), Infrared Technology and Applications XXXVIII, Baltimore, U.S.A., Apr. 2012.

- N. Narang and T. Bourlai, "Can we match ultraviolet face images against their visible counterparts?, Algorithms and Technologies for Multispectral, Hyperspectral, and Ultraspectral Imagery XXI, SPIE (Defense + Security), Baltimore, MD, April 2015.

- N. Narang and T. Bourlai, "Gender and Ethnicity Classification using Deep Learning in Heterogeneous Face Recognition”, 9th IAPR International Conference on Biometrics (ICB), Halmstad, Sweden, June 13-16, 2016. 
- N. Narang, T. Bourlai,"Deep Features based Classification of Multi-spectral Images when using for Face Recognition in the SWIR Band", CVPR Women in Computer Vision workshop, Las Vegas, NV, USA, June 2016.

- N. Narang and T. Bourlai, "On the Effectiveness of Statistical Hypothesis Testing in IR-based FR in Heterogeneous Environments, IEEE ASONAM (SNAST), August 2016.

- N. Narang, T. Bourlai,“Learning deep features for hierarchical classification of mobile database in heterogeneous environment, 12th IEEE International Conference on Automatic Face and Gesture Recognition (FG), Washington, DC, June 2017.

\section{Invited Book Chapter:}

N. Narang and T. Bourlai, "Learning Deep Features for Situation Classification in Short Wave Infrared Band-based Face Recognition for Surveillance Applications”, Surveillance in Action, Springer Publishers 2017. 


\section{Bibliography}

[1] J. C. Klontz and A. K. Jain, "A case study on unconstrained facial recognition using the boston marathon bombings suspects," in Technical Report MSU-CSE-13-4, pp. 1-8, May 2013.

[2] S. Z. Li and A. K. Jain, Handbook of Face Recognition. Springer, 2011.

[3] N. Narang and T. Bourlai, "Face recognition in the SWIR band when using single sensor multi-wavelength imaging systems," Image and Vision Computing, vol. 33, pp. 26 - 43, January 2015.

[4] B. A. Draper, K. Baek, M. S. Bartlett, and J. R. Beveridge, "Recognizing faces with pca and ica," in Computer Vision and Image Understanding, Special Issue on Face Recognition, pp. 115-137, 2003.

[5] J. Lu, K. N. Plataniotis, and A. N. Venetsanopoulos, "Regularization studies on lda for face recognition," in International Conference on Image Processing ICIP, pp. 63-66, 2004.

[6] S. Z. Li, R. Chu, S. Liao, and L. Zhang, "Illumination invariant face recognition using near-infrared images," IEEE Transactions on Pattern Analysis and Machine Intelligence, vol. 29, pp. 627-639, April 2007.

[7] T. Bourlai, J.Dollen, N. Mavridis, and C. Kolanko, "Evaluating the effciency of a nighttime, middle-range infrared sensor for applications in human detection and recognition," in Proceedings of SPIE Defense, Security, and Sensing, (Baltimore, USA), pp. 83551B83551B-12, May 2012. 
[8] N. Narang, "Designing a single sensor multi-wavelength facial-based acquisition system in swir band," Master's thesis, West Virginia University, USA, 2013.

[9] T. Bourlai, "Mid-wave ir face recognition systems," in SPIE Newsroom Magazine - Defense \& Security, pp. 1-3, February 2013.

[10] T. Bourlai, "Short-wave infrared for face-based recognition systems," in SPIE Newsroom Magazine - Defense \& Security, pp. 1-2, April 2012.

[11] W. Zhao, R. Chellappa, A. Rosenfeld, and P. Phillips, "Face recognition: A literature survey," ACM Computing Surveys, pp. 399-458, 2006.

[12] Y. Yao, B. R. Abidi, R. Besma, N. D. Kalka, N. A. Schmid, M. A. Abidi, and A. Mongi., "Improving long range and high magnification face recognition: Database acquisition, evaluation, and enhancement," Computer Vision and Image Understanding, vol. 111, 2007.

[13] S. G. Kong, J. Heo, B. R. Abidi, J. Paik, and M. A. Abidi, "Recent advances in visual and infrared face recognitiona review," Computer Vision and Image Understanding, vol. 97, no. 1 , pp. 103-135, 2005.

[14] J. Kang, A. Borkar, A. Yeung, N. Nong, M. Smith, and M. Hayes, "Short wavelength infrared face recognition for personalization," in International Conference on Image Processing ICIP, pp. 2757-2760, Oct 2006.

[15] B. E. Lemoff, R. B. Martin, M. Sluch, K. M. Kafka, W. McCormick, and R. Ice, "Longrange night/day human identification using active-swir imaging," in Proc. SPIE Infrared Technology and Applications XXXIX, vol. 8704, pp. 87042J-87042J-8, June 2013.

[16] M. Bertozzi, R. I. Fedriga, A. Miron, and J. L. Reverchon, "Pedestrian detection in poor visibility conditions: would swir help?," in International Conference on Image Analysis and Processing, pp. 229-238, 2013.

[17] F. Nicolo and N. A. Schmid, "Long range cross-spectral face recognition: matching swir 
against visible light images," Information Forensics and Security, IEEE Transactions on, vol. 7, no. 6, pp. 1717-1726, 2012.

[18] R. B. Martin, M. Sluch, K. M. Kafka, R. Ice, and B. E. Lemoff, “Active-SWIR signatures for long-range night/day human detection and identification," in SPIE Defense, Security, and Sensing, pp. 87340J-87340J, International Society for Optics and Photonics, 2013.

[19] Z. X. Cao and N. A. Schmid, "Recognition performance of cross-spectral periocular biometrics and partial face at short and long standoff distance," Open Trans. Inf. Process, vol. 1, pp. 20-32, 2014.

[20] B. DeCann, A. Ross, and J. Dawson, "Investigating gait recognition in the short-wave infrared (swir) spectrum: dataset and challenges," in Proc. SPIE Biometric and Surveillance Technology for Human and Activity Identification X, vol. 8712, pp. 87120J87120J-16, May 2013.

[21] M. H. Ettenberg, "A little night vision-InGaAs shortwave infrared emerges as key complement to IR for military imaging.," Advanced Imaging-Fort Atkinson, vol. 20, no. 3, pp. 29-33, 2005.

[22] N. Narang and T. Bourlai, "Gender and Ethnicity classification using deep learning in heterogeneous face recognition," in 2016 International Conference on Biometrics (ICB), pp. 1-8, June 2016.

[23] D. M. N. Narang, M. Martin and T. Bourlai, "Learning Deep Features for hierarchical classification of mobile phone face datasets in heterogeneous environments," in 12th IEEE Conference on Automatic Face and Gesture Recognition (FG), pp. 1-8, May 2017.

[24] N. Kalka, T. Bourlai, B. Cukic, and L. Hornak, "Cross-spectral Face recognition in Heterogeneous Environments: A Case Study on Matching Visible to Short-wave Infrared Imagery," in International Joint Conference on Biometrics, pp. 1-8, 2011.

[25] A. Reza, "Realization of contrast limited adaptive histogram equalization (clahe) for real-time image enhancement," VLSI Signal Processing, vol. 38, pp. 35-44, 2004. 
[26] M. Liu, W. Xie, X. Chen, Y. Ma, Y. Guo, J. Meng, Z. Yuan, and Q. Qin, "Heterogeneous face biometrics based on guassian weights and invariant features synthesis," in Proceedings of IEEE 2nd International Conference on Computing, Control and Industrial Engineering (CCIE), vol. II, pp. 374-377, Aug 2011.

[27] J. Chen, D. Yi, J. Yang, G. Zhao, S. Z. Li, and M. Pietikainen, "Learning mappings for face synthesis from near infrared to visual light images," in Proceedings of IEEE Conference on Computer Vision and Pattern Recognition (CVPR), pp. 156-163, 2009.

[28] B. Klare and A. K. Jain, "Heterogeneous face recognition: Matching NIR to Visible light images," in Proceedings of 20th International Conference on Pattern Recognition (ICPR), (Washington, DC, USA), pp. 1513-1516, 2010.

[29] H. Maeng, H. C. Choi, U. Park, S. W. Lee, and A. K. Jain, "Nfrad: Near-infrared face recognition at a distance," in Proceedings of International Joint Conference on Biometrics (IJCB), pp. 1-7, IEEE, 2011.

[30] D. Yi, R. Liu, R. Chu, Z. Lei, and S. Z. Li, "Face matching between near infrared and visible light images," in Advances in Biometrics, vol. 4642 of Lecture Notes in Computer Science, pp. 523-530, Springer, 2007.

[31] M. Hyunju, S. Liao, D. Kang, S. W. Lee, and A. K. Jain, "Nighttime face recognition at long distance: Cross-distance and cross-spectral matching," in Proceedings of Computer VisionACCV'12, vol. II, (Berlin, Heidelberg), pp. 708-721, 2013.

[32] R. Wang, J. Yang, D. Yi, and S. Li, "An analysis-by-synthesis method for heterogeneous face biometrics," in Advances in Biometrics, vol. 5558, pp. 319-326, Springer, 2009.

[33] Z. Zhang, Y. Wang, and Z. Zhang, "Face synthesis from near-infrared to visual light via sparse representation," in Proceedings of IEEE International Joint Conference on Biometrics, (IJCB), (Washington, DC, USA), pp. 1-6, October 2011.

[34] J. Y. Zhu, W. S. Zheng, and J. Lai, "Transductive vis-nir face matching," in Proceedings of 19th IEEE International Conference on Image Processing (ICIP), pp. 1437-1440, Sept 2012. 
[35] D. Goswami, C. H. Chan, D. Windridge, and J. Kittler, "Evaluation of face recognition system in heterogeneous environments (visible vs NIR)," in Proceedings of IEEE International Conference on Computer Vision (ICCV) Workshops, vol. 5558, (Barcelona, Spain), pp. 2160-2167, November 2011.

[36] R. Raghavendra, B. Dorizzi, A. Rao, and G. H. Kumar, "Particle swarm optimization based fusion of near infrared and visible images for improved face verification," Pattern Recognition, vol. 44, no. 2, pp. 401-411, 2011.

[37] F. Omri, S. Foufou, and M. Abidi, "Pixel level fusion of multispectral face images: Short review," in Proceedings of 7th IEEE Gulf Cooperation Council (GCC) Conference and Exhibition, (Doha Qatar), pp. 595-600, Nov 2013.

[38] F. Omri, S. Foufou, and M. Abidi, "Nir and visible image fusion for improving face recognition at long distance," in Image and Signal Processing, pp. 549-557, Springer, 2014.

[39] D. Kang, H. Han, A. K. Jain, and S. W. Lee, "Nighttime face recognition at large standoff: Cross-distance and cross-spectral matching," Pattern Recognition, vol. 47, no. 12, pp. 3750-3766, 2014.

[40] T. Bourlai and B. Cukic, "Multi-spectral face recognition: Identification of people in difficult environments," in Proceedings of IEEE International Conference on Intelligence and Security Informatics (ISI), (Washington, U.S.A), pp. 196-201, June 2012.

[41] J. Mansanet, A. Albiol, and R. Paredes, "Local deep neural networks for gender recognition," Pattern Recognition Letters, vol. 70, pp. 80-86, 2016.

[42] A. K. Jain, S. C. Dass, and K. Nandakumar, "Can soft biometric traits assist user recognition?," in Proceedings of SPIE, vol. 5404, pp. 561-572, 2004.

[43] D. Reid, S. Samangooei, C. Chen, M. Nixon, and A. Ross, "Soft biometrics for surveillance: an overview." 2012. 
[44] M. S. Nixon, P. L. Correia, K. Nasrollahi, T. B. Moeslund, A. Hadid, and M. Tistarelli, “On soft biometrics,” Pattern Recognition Letters, vol. 68, pp. 218-230, 2015.

[45] D. Mery and K. Bowyer, "Automatic facial attribute analysis via adaptive sparse representation of random patches," Pattern Recognition Letters, 2015.

[46] U. Park and A. K. Jain, "Face matching and retrieval using soft biometrics," Information Forensics and Security, IEEE Transactions on, vol. 5, no. 3, pp. 406-415, 2010.

[47] G. Givens, J. R. Beveridge, B. A. Draper, and D. Bolme, "A statistical assessment of subject factors in the pca recognition of human subjects," in Proceedings of CVPR Workshop: Statistical Analysis in Computer Vision, vol. 733, 2003.

[48] J. R. Lyle, P. E. Miller, S. J. Pundlik, and D. L. Woodard, "Soft biometric classification using periocular region features," in Biometrics: Theory Applications and Systems (BTAS), Fourth IEEE International Conference on, pp. 1-7, Sept 2010.

[49] C. Chen and A. Ross, "Local gradient Gabor pattern (LGGP) with applications in face recognition, cross-spectral matching, and soft biometrics," in Proc. SPIE Biometric and Surveillance Technology for Human and Activity Identification X, (Baltimore, Maryland, USA), pp. 87120R-87120R, 2013.

[50] C. Chen and A. Ross, "Evaluation of gender classification methods on thermal and nearinfrared face images," in IEEE International Joint Conference on Biometrics, IJCB, (Washington, DC, USA), pp. 1-8, October 2011.

[51] S. Lagree and K. W. Bowyer, "Predicting ethnicity and gender from iris texture," in Technologies for Homeland Security (HST), 2011 IEEE International Conference on, pp. 440-445, Nov 2011.

[52] G. Levi and T. Hassner, "Age and gender classification using convolutional neural networks," in IEEE Conf. on Computer Vision and Pattern Recognition (CVPR) workshops, June 2015. 
[53] A. Vailaya, M. A. T. Figueiredo, A. K. Jain, and H. J. Zhang, "Image classification for content-based indexing," Image Processing, IEEE Transactions on, vol. 10, no. 1, pp. 117-130, 2001.

[54] L. Gupta, V. Pathangay, A. Patra, A. Dyana, and S. Das, "Indoor versus Outdoor scene classification using probabilistic neural network," EURASIP Journal on Advances in Signal Processing, vol. 2007, no. 1, pp. 1-10, 2006.

[55] A. Payne and S. Singh, "A benchmark for Indoor/Outdoor scene classification," in Pattern Recognition and Image Analysis, pp. 711-718, 2005.

[56] A. Krizhevsky, I. Sutskever, and G. E. Hinton, "Imagenet classification with deep convolutional neural networks," in Advances in Neural Information Processing Systems 25, pp. 1097-1105, 2012.

[57] S. Sarkar, V. M. Patel, and R. Chellappa, "Deep feature-based face detection on mobile devices," arXiv preprint arXiv:1602.04868, 2016.

[58] E. Nowak, F. Jurie, and B. Triggs, "Sampling strategies for bag-of-features image classification," in European Conference on Computer Vision, Springer, 2006.

[59] H. Jegou, M. Douze, and C. Schmid, "Improving bag-of-features for large scale image search,” International Journal of Computer Vision, vol. 87, no. 3, pp. 316-336, 2010.

[60] S. T. Namin and L. Petersson, "Classification of materials in natural scenes using multispectral images.," in IROS, pp. 1393-1398, IEEE, 2012.

[61] J. Ice, N. Narang, C. Whitelam, N. Kalka, L. Hornak, J. Dawson, and T. Bourlai, "SWIR imaging for facial image capture through tinted materials," in SPIE Defense, Security, and Sensing, pp. 83530S-83530S, International Society for Optics and Photonics, 2012.

[62] G. V. G. V. Veres, L. Gordon, J. N. Carter, and M. S. Nixon, "What image information is important in silhouette-based gait recognition?," in Proceedings of International Conference on Computer Vision \& Pattern Recognition (CVPR), vol. 2, pp. 776-782, 2004. 
[63] B. Guo and M. S. Nixon, "Gait feature subset selection by mutual information," Systems, Man and Cybernetics, Part A: Systems and Humans, IEEE Transactions on, vol. 39, no. 1 , pp. 36-46, 2009.

[64] J. D. Lee, C. Y. Lin, and C. H. Huang, "Novel features selection for gender classification," in Mechatronics and Automation (ICMA), 2013 IEEE International Conference on, pp. 785-790, 2013.

[65] N. Dalal and B. Triggs, "Histograms of oriented gradients for human detection," in Proceedings of International Conference on Computer Vision \& Pattern Recognition (CVPR), vol. 2, pp. 886-893, June 2005.

[66] E. Franceschi, F. Odone, F. Smeraldi, and A. Verri, "Feature selection with nonparametric statistics," in Image Processing, 2005. ICIP 2005. IEEE International Conference on, vol. 1, pp. I-325, 2005.

[67] A. Kumar and D. Zhang, "Biometric recognition using feature selection and combination," in Audio-and Video-Based Biometric Person Authentication, pp. 813-822, 2005.

[68] T. Bourlai, N. Mavridis, and N. Narang, "On designing practical long range near infrared-based face recognition systems," Image and Vision Computing, vol. 52, p. 2541, May 2016.

[69] N. Narang and T. Bourlai, "Gender and ethnicity classification using deep learning in heterogeneous face recognition," in 9th IAPR International Conference on Biometrics ICB, (Halmstad, Sweden), June 2016.

[70] H. Maeng, S. Liao, D. Kang, S.-W. Lee, , and A. K. Jain, "Nighttime face recognition at long distance: Cross-distance and cross-spectral matching," in Asian Conference on Computer Vision Conference on Computer Vision (ACCV), pp. 5-9, 2012.

[71] D. Kang, H. Han, A. K. Jain, and S.-W. Lee, "Nighttime face recognition at large standoff: Cross-distance and cross-spectral matching," Pattern Recognition, vol. 47 (12), pp. 3750-3766, 2014. 
[72] J. M. Dawson, S. C. Leffel, C. Whitelam, and T. Bourlai, "Collection of multispectral biometric data for cross-spectral identification applications," in Face Recognition Across the Imaging Spectrum, pp. 21-46, Springer, 2016.

[73] T. Martin, R. Brubaker, P. Dixon, M. A. Gagliardi, and T. Sudol, “640x512 InGaAs focal plane array camera for visible and SWIR imaging," in Defense and Security, pp. 12-20, International Society for Optics and Photonics, 2005.

[74] T. Bourlai, N. Narang, B. Cukic, and L. Hornak, "On Designing a Swir MultiWavelength Facial-based Acquisition System," in Proc. SPIE Infrared Technology and Applications XXXVIII, vol. 8353, (Baltimore, USA), pp. 83530R-83530R-14, May 2012.

[75] D. S. Bolme, J. R. Beveridge, M. L. Teixeira, and B. A. Draper, "The CSU face identification evaluation system: Its purpose, features and structure," in Proc. International Conference on Vision Systems, (Graz, Austria), pp. 304 - 311, April 2003.

[76] S. Murala, R. P. Maheshwari, and R. Balasubramanian, "Local tetra patterns: a new feature descriptor for content-based image retrieval," IEEE Transactions on Image Processing, vol. 21, no. 5, pp. 2874-2886, 2012.

[77] X. Tan and B. Triggs, "Enhanced local texture feature sets for face recognition under difficult lighting conditions," IEEE Transactions on Image Processing, vol. 19, pp. 16351650, June 2010.

[78] L. Shen and L. Bai, "A review on gabor wavelets for face recognition," Pattern Anal. Appl., vol. 9, pp. 273-292, oct 2006.

[79] A. Vedaldi and K. Lenc, "MatConvNet - Convolutional Neural Networks for MATLAB," in Proceedings of the 23rd ACM International Conference on Multimedia, (New York, NY, USA), pp. 689-692, 2015.

[80] O. M. Parkhi, A. Vedaldi, and A. Zisserman, "Deep face recognition," Proceedings of the British Machine Vision, vol. 1, no. 3, p. 6, 2015. 
[81] F. Gilad and F. Raanan, "Image and video upscaling from local self examples," $A C M$ Transactions on Graphics (TOG), vol. 30, April 2011.

[82] T. Bourlai, A. Ross, and A. Jain, "On matching digital face images against scanned passport photos," in Proceedings of First IEEE International Conference on Biometrics, Identity and Security (BIDS), (Tampa, USA), September 2009.

[83] V. Struc, “The inface toolbox for illumination invariant face recognition," February 2012.

[84] L. Sirovich and M. Kirby, "Application of the Karhunen-Loeve procedure for the characterization of human faces," Transactions on Pattern Analysis and Machine Intelligence, vol. 12, no. 1, pp. 103-108, 1990.

[85] M. Turk and A. Pentland, "Eigenfaces for recognition," Journal of Cognitive Neuroscience, vol. 3, no. 1, pp. 71-86, 1991.

[86] A. P. Devijver and J. Kittler, Pattern recognition: A statistical approach. Prentice-Hall, Englewood Cliffs, N. J., 1982.

[87] P. Belhumeur, J. Hespanha, and D. J. Kriegman, "Eigenfaces vs. fisherfaces: Recognition using class specific linear projection," Transactions on Pattern Analysis and Machine Intelligence, vol. 19, pp. 45-58, 1996.

[88] M. Teixeira, “The bayesian intrapersonal/extrapersonal classifier,” Master's thesis, Colorado State University, Fort Collins, Colorado 80523 U.S.A., 2003.

[89] D. T. Campbell and J. C. Stanley, Experimental and quasi-experimental designs for research. Ravenio Books, 2015.

[90] R. Hyman, "Quasi-experimentation: Design and analysis issues for field settings (book)," Journal of Personality Assessment, vol. 46, no. 1, pp. 96-97, 1982.

[91] W. MICHAEL, "Campbell, dt-experimental and quasi-experimental designs for research," 1967. 
[92] D. T. Campbell, "Reforms as experiments.," American psychologist, vol. 24, no. 4, p. $409,1969$.

[93] S. Dey and D. Samanta, Unimodal and Multimodal Biometric Data Indexing. 2014.

[94] M. Hamill and K. G. Popstojanova, "Common trends in software fault and failure data," IEEE Transactions on Software Engineering, vol. 35, no. 4, pp. 484-496, 2009.

[95] A. S. Sayyad, K. G. Popstojanova, T. Menzies, and H. Ammar, "On parameter tuning in search based software engineering: A replicated empirical study," in Replication in Empirical Software Engineering Research (RESER), 2013 3rd International Workshop on, pp. 84-90, IEEE, 2013.

[96] S. Krishnan, C. Strasburg, R. R. Lutz, K. G. Popstojanova, and S. K. Dorman, "Predicting failure-proneness in an evolving software product line," Information and Software Technology, vol. 55, no. 8, pp. 1479-1495, 2013.

[97] A. Abaza and T. Bourlai, "On ear-based human identification in the mid-wave infrared spectrum," Image and Vision Computing, vol. 31, no. 9, pp. 640-648, 2013.

[98] C. Whitelam, Z. Jafri, and T. Bourlai, "Multispectral eye detection: A preliminary study," in Pattern Recognition (ICPR), 2010 20th International Conference on, pp. 209212, IEEE, 2010.

[99] C. E. Thomaz and G. A. Giraldi, "A new ranking method for principal components analysis and its application to face image analysis," Image and Vision Computing, vol. 28, no. 6, pp. 902-913, 2010.

[100] D. Hond and L. Spacek, "Distinctive descriptions for face processing.," in $B M V C$, no. 0.2 , pp. 0-4, 1997.

[101] B. Klare, M. Burge, J. Klontz, R. V. Bruegge, and A. K. Jain, "Face recognition performance: Role of demographic information," Information Forensics and Security, IEEE Transactions on, vol. 7, pp. 1789-1801, Dec 2012. 
[102] G. Polina and F. Bruce, "Permutation tests for classification: Towards statistical significance in image-based studies," in Information processing in medical imaging (IPMI), vol. 2732 of Lecture Notes in Computer Science, pp. 330-341, Springer, 2003.

[103] D. D. Boos and C. Brownie, "Anova and rank tests when the number of treatments is large," Statistics and Probability Letters, vol. 23, no. 2, pp. 183 - 191, 1995.

[104] N. Razali and Y. B. Wah, "Power comparisons of shapiro-wilk, kolmogorov-smirnov, lilliefors and anderson-darling tests," Journal of Statistical Modeling and Analytics, vol. 2, no. 1, pp. 21-33, 2011.

[105] J. L. Gastwirth, Y. R. Gel, and W. Miao, "The impact of levenes test of equality of variances on statistical theory and practice," Statistical Science, vol. 24, 082009.

[106] W. H. Kruskal and W. A. Wallis, "Use of ranks in one-criterion variance analysis," Journal of the American statistical Association, vol. 47, no. 260, pp. 583-621, 1952.

[107] G. D. Ruxton and G. Beauchamp, “Time for some a priori thinking about post hoc testing," Behavioral Ecology, vol. 19, no. 3, pp. 690-693, 2008.

[108] L. I. Kuncheva and C. J. Whitaker, "Measures of diversity in classifier ensembles and their relationship with the ensemble accuracy," Machine learning, vol. 51, no. 2, pp. 181207, 2003.

[109] A. A. Ross, K. Nandakumar, and A. K. Jain, Handbook of multibiometrics, vol. 6. 2006.

[110] J. Aghajanian and S. Prince, "Face pose estimation in uncontrolled environments.," in Proceedings of the British Machine Vision Conference, BMVC, vol. 1, pp. 1-11, September 2009.

[111] P. Viola and M. J. Jones, "Robust real-time face detection," International journal of computer vision, vol. 57, no. 2, pp. 137-154, 2004.

[112] J. Whitehill and J. R. Movellan, "A discriminative approach to frame-by-frame head pose estimation," in Int. Conf. Automatic Face and Gesture Recognition, 2008. 